\author{
Dissertation \\ submitted to the \\ Combined Faculties for the Natural Sciences and for \\ Mathematics \\ of the Ruperto-Carola University of Heidelberg, Germany \\ for the degree of \\ Doctor of Natural Sciences
}

presented by

Diplom-Physiker José Fiestas

born in Lima, Perú

Oral examination: 01. February 2006 



\section{Dynamical evolution of rotating globular clusters with embedded black holes}

Referees: Prof. Dr. Rainer Spurzem

Prof. Dr. Stefan Wagner 



\section{Abstract}

In this dissertation evolution of self-gravitating dense stellar systems (e.g. globular clusters, galactic nuclei) with embedded black holes is investigated, motivated by observational evidences of the existence of central dark objects in these systems. The interaction between the stellar and black hole component is followed in a way, different from most other investigations in this field, as flattening of the system due to differential rotation is allowed. The axisymmetric system is modelled using 2-dimensional, in energy and z-component of angular momentum, Fokker Planck numerical methods. The interplay between velocity diffusion due to relaxation and black hole star accretion is followed together with cluster rotation. The results show how angular momentum transport and star accretion support the development of central rotation in relaxation time scales. Gravogyro and gravothermal instabilities conduce the system to a faster evolution leading to shorter collapse times with respect to models without black hole, and a faster cluster dissolution in the galactic tidal field of a parent galaxy. As a further application, two-dimensional distribution (in the meridional plane) of kinematical and structural parameters (density, dispersions, rotation) are reproduced, covering a wide range of ages, rotation, concentrations and ellipticities, with the aim to enable the use of set of models for comparison with observational data.

\section{Zusammenfassung}

Diese Dissertation befasst sich mit der Untersuchung der Entwicklung selbstgravitierender dichter Sternsystemen (Kugelsternhaufen, aktive Galaxienkerne) mit einem zentralen Schwarzes-Loch, motiviert durch Beochachtungshinweise über die Existenz Schwarze Löcher in diesen Sternsysteme. Die Wechselwirkung zwischen Stern- und

Schwarzes-Loch-Komponente is verfolgt von einem Standpunkt, der sich von den meisten Untersuchungen in diesem Gebiet unterscheidet, da eine Abflachung des Systems is durch differentielle Rotation ermöglicht. Das axysimmetrische System is simuliert unter Verwendung zwei-dimensionaler, in Energie und Drehimpuls in z-Richtung, Fokker-Planck numerische Methoden. Die Wechselwirkung zwischen Diffusionprozesse und Sternenakkretion durch das Schwarze Loch ist verfolgt, unter dem Einfluss von Rotation. Die Ergebnisse zeigen wie Drehimpulstransport und Sternenakkretion unterstützen die Entstehung zentraler Rotation in Zeitskalen der Relaxation. Gravogyro und gravothermische Instabilitäten führen zu einer schnelleren Entwicklung in kurzen Kollapszeiten in Bezug auf Modelle ohne Schwarzes-Loch, und schliesslich zu einer schnelleren Auflösung des Sternhaufens im Tidenfeld der Muttergalaxie. Eine weitere Anwendung ist die Erstellung zweiDimensionale Verteilungen (in der meridionalen Ebene) kinematischer und Strukturparametern (Dichte, Dispersion, Rotation) in einen breiten Alter-, Rotation-, Konzentration- und Elliptizitäts-Wertebereich, mit dem Ziel der Verwendung theoretischer Modelle zum Vergleich mit Beobachtungsdata. 



\section{Dedication}

This dissertation is dedicated to Wendy, my wife, who supports and encourages me every day, and to my parents, Sila and Reynaldo, who showed me how to persevere through life's challenges. 



\section{Contents}

1 Introduction 1

1.1 Globular cluster parameters . . . . . . . . . . . . . . . . . 3

1.2 Globular clusters in the Local Group . . . . . . . . . . . . . . . . . . . . 4

1.3 Flattening and rotation . . . . . . . . . . . . . . . . 9

1.4 Black holes in dense stellar systems . . . . . . . . . . . . . . . . . 11

1.5 Aims of this study . . . . . . . . . . . . . . . . . . 14

2 Theoretical dynamics $\quad 17$

2.1 Numerical Methods . . . . . . . . . . . . . . . . . . . . . . . . . . . . . . . 18

2.2 Rotating Models . . . . . . . . . . . . . . . . . . . . . . . . 19

2.3 Black Hole Models . . . . . . . . . . . . . . . . . . . . . 22

2.4 Tidal fields . . . . . . . . . . . . . . . . . . . . . . . 25

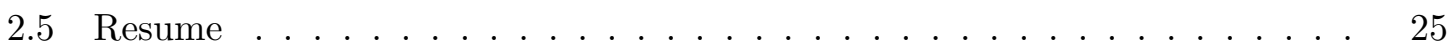

3 Model 27

3.1 Fokker Planck equation . . . . . . . . . . . . . . . . . . . . . . . 28

3.1 .1 Equations and assumptions . . . . . . . . . . . 28

3.1 .2 Integrals of motion . . . . . . . . . . . . . . . . 30

3.1 .3 Units and $E, J_{z}$-grid $\ldots \ldots \ldots \ldots \ldots \ldots \ldots \ldots$

3.2 Difussion and loss-cone limit . . . . . . . . . . . . . . . 33

3.2 .1 Orbit average and flux conservation . . . . . . . . . 33

3.2 .2 Loss cone accretion . . . . . . . . . . . . . . . . . 36

3.2 .3 Tidal limit . . . . . . . . . . . . . . . . . . . . . . 41

3.2 .4 Ellipticity . . . . . . . . . . . . . . . . . . . 42

3.3 Numerical method . . . . . . . . . . . . . . . . . . . . . . 43

3.3.1 Fokker-Planck step and loss-cone diffusion . . . . . . . . . . . . . . 43

3.3.2 Vlasov Step and calculation of the cluster potential . . . . . . . . . . 44

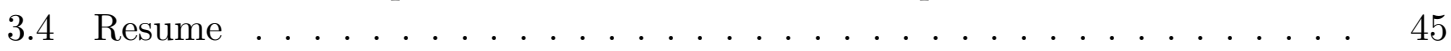

$\begin{array}{lll}4 & \text { Results } & 47\end{array}$

4.1 Initial conditions . . . . . . . . . . . . . . . . . . . 48

4.2 Evolution of cluster structure . . . . . . . . . . . . . . . 51

4.3 Rotation and angular momentum . . . . . . . . . . . . . . . 72

4.4 Distribution function . . . . . . . . . . . . . . . . . . . 84

4.5 Observational data comparison . . . . . . . . . . . . . . . . . 94

$\begin{array}{lll}5 & \text { Conclusions } & 97\end{array}$ 
Appendix

Vita

125 


\section{List of Tables}

1.1 Globular cluster observational data . . . . . . . . . . . . . . . 5 5

1.2 Rotational parameters of globular clusters . . . . . . . . . . . . . . . 10

4.1 Initial model classification . . . . . . . . . . . . . . . . . . . . . . . 49

4.2 Initial model parameters . . . . . . . . . . . . . . . . . 50

4.3 Initial BH parameters . . . . . . . . . . . . . . . . . . . . 51

4.4 Collapse parameters . . . . . . . . . . . . . . . . . 62

4.5 Description of mass parameters . . . . . . . . . . . . . . 71

4.6 Rotation parameters at collapse . . . . . . . . . . . . . 80 



\section{List of Figures}

1.1 Distribution of globular clusters in the Milky Way. Some clusters of Table (1.1) are illustrated. M5, $\omega \mathrm{Cen}$ and 47Tuc are at distances between $6-7 \mathrm{kpc}$; NGC 5286 is at $8.4 \mathrm{kpc}$; M15 and NGC2808 are located at 10-11 kpc from the galactic center. The Milky Way picture was taken by the COBE satellite and shows the plane of our Galaxy in infrared light. The thin disk is clearly apparent, with stars appearing white and interstellar dust appearing red. It is overploted by globular cluster positions taken from the Harris Catalog (Harris et al., 1996, February 2003 revision). . . . . .

1.2 HST images of M31 GCs (G11, G64 and G302) analyzed in Rich et al. 2005. All are $20^{\prime \prime} \times 20^{\prime \prime}(=80 \mathrm{pc})$ sub-rasters (upper figures). The corresponding CMDs are shown below. Each cluster is labeled with its name, metallicity, and number of stars displayed. . . . . . . . . . . . . . . . . . .

1.3 Chandra image of the stellar mass black hole candidate at the position of the quiescent X-ray nova XTE J1118+480 (central bright point). The spikes and rays extending from the bright point are instrumental artifacts. From McClintock et al. (2003). $M_{\mathrm{BH}}<10 M_{\odot} \ldots \ldots \ldots \ldots \ldots \ldots \ldots \ldots$

1.4 Chandra Image of the black hole discovered in a dense star cluster about 600 light years from the center of the galaxy M82. Astronomers found that the mass of the black hole must be greater than $500 M_{\odot} \ldots \ldots \ldots \ldots$

1.5 A composite X-ray (blue) from Karovska et al. (2002); radio (pink and green) from Van Gorkom et al. (1994), and optical (orange and yellow) from Digitized Sky Survey U.K. Schmidt Image/STScI; image of the galaxy Centaurus A. A broad band of dust and cold gas is bisected at an angle by opposing jets of high-energy particles blasting away from the supermassive black hole in the nucleus $\left(M_{\mathrm{BH}} \sim 1-3 \cdot 10^{8} M_{\odot}\right)$

2.1 Comparative study of the dynamical evolution of a model star cluster at collapse time. The upper panel shows the time and mass at core collapse and the lower panel, the radii at core collapse. Filled symbols represent N-Body results, empty symbols are results of Fokker-Planck methods, and the stars are for gaseous models. From Heggie (2003). . . . . . . . . . . . . . . . . . .

3.1 Evolution of areas $A\left(E, J_{\mathrm{z}}\right)$ (blue coloured) for an initially rotating flattened model $\left(6.0,0.6,5 \cdot 10^{-6}\right)$ using always the same E-grid cell (number 30 ) and $J_{\mathrm{z}}$-grid cell (number 40). The third integral is approximated by $J=\left(J_{\mathrm{z}}+J_{\mathrm{z}}^{\max }\right) / 2$ and the $A\left(E, J_{\mathrm{z}}, J^{2}\right)$ 's are overploted (vertical line areas) $\ldots \ldots \ldots$ 
3.2 Schematic diagram of the numerical $X Y$-grid and definition of the loss cone. Half of the grid is shown in the left side of the figure. The other lower half corresponds to negative values of $\mathrm{Y}$ and is symmetric with respect to the $Y=0$-axis. The blue shaded area represents the loss cone in $X-Y$-space, and is limited by $Y_{\min }$. Stars are able to go into and out of it through angular momentum diffusion, as shown in the right side of the figure, for one grid cell. Energy diffusion to the loss cone in the horizontal direction is neglected, but they are able to escape the cluster through the tidal boundary at $X\left(E_{\text {tid }}\right) \ldots \ldots \ldots \ldots \ldots$

3.3 The panel shows the definition of critical energy $\left(E_{\text {crit }}\right)$ using data of a model with initial moderate concentration $W_{0}=6.0$ and high rotation $\omega_{0}=0.9$. The red curve is the loss-cone limit as defined in Eq. (3.24), and the black line, the averaged angular momentum diffusion at constant energy. The curves intersect at $\left(E_{\text {crit }} \ldots \ldots\right.$. . .

$4.1 f\left(J_{z}\right)$, at constant energy $E$, for an initial model $\left(W_{0}=0.6, \omega_{0}=0.9\right)$. From top to bottom the curves have smaller $|E|$, listed on the left column. Grid dimension is $(10061)$ in $\left(E, J_{\mathrm{z}}\right)$-space used to construct the models. . . . .

4.2 Equatorial density profile $(z=0)$ for models $W_{0}=6.0$ and $W_{0}=3.0$, and initial rotation $\omega_{0}=0.6$ (Models A3 and B3) for different times (in parenthesis), given in units of initial half-mass relaxation time $\left(t_{\text {rhi }}\right)$. The dashed line shows the $-7 / 4$ slope and the dot-dashed lines the evolutionary profiles. The location of $r_{\mathrm{a}}$ is shown as squares. The $\mathrm{BH}$ mass is initially $5 \cdot 10^{-6}$ and $M_{\mathrm{bh}} \sim 0.01 M_{\mathrm{cl}}(0)$ as the curve achieves the steady state (solid sloped line). . . . . . . . . . .

4.3 Same as Fig. 4.2 for models of $W_{0}=9.0$ and initial rotation $\omega_{0}=0.3$ (Model C2).

4.4 Evolution of density distribution in the meridional plane for a model B4. Double logarithmic cylindrical coordinates $(\varpi, z)$ are used. Lighter zones represent higher isodensity contours. The time is given in units of initial half-mass relaxation time

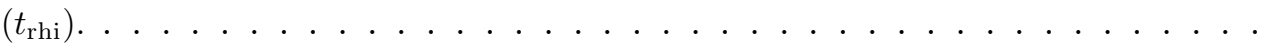

4.5 Equatorial profile $(z=0)$ of 1D total velocity dispersion as in Figures (4.2) and (4.3) for the density (Models A3 and B3). The dashed line shows the $-1 / 2$ slope and the dot-dashed lines the evolutionary profiles. The location of $r_{\mathrm{a}}$ is shown as squares. The curve achieves the steady state (solid sloped line) close to the collapse. . . . .

4.6 Same as Fig. 4.5, for the model $W_{0}=9.0$ and initial rotation $\omega_{0}=0.3$ (Model $\mathrm{C} 2$ ). Note the faster evolution and lower final dispersions in the center as in the less concentrated models. . . . . . . . . . . . . . . . .

4.7 1D total velocity dispersion for the final state of model of Fig. 4.5. The dashed line shows the $-1 / 2$ slope and the dot-dashed line the initial total 1D dispersion profile. Note the higher tangential component $\left(\sigma_{\phi}\right)$ in the central regime (red line) and the lower radial component $\left(\sigma_{r}\right)$ due to $\mathrm{BH}$ accretion of low- $J_{z}$ radial orbits (yellow line). 59

4.8 Anisotropy, defined as $A \equiv 1-\frac{\sigma_{\phi}{ }^{2}}{\sigma_{\mathrm{r}}{ }^{2}}$, against radius for the same model of Fig. 4.7. The dashed line represents the zero-line and the dot-dashed line the initial profile. $\quad 59$

4.9 Evolution of central density for models of $W_{0}=6.0$ and initial rotation $\omega_{0}=0.0,0.3,0.6,0.9,1.2$. The time is given in units of initial half-mass relaxation time $\left(t_{\mathrm{rhi}}\right) \ldots \ldots 60$

4.10 Evolution of central density for models of $W_{0}=3.0$ and initial rotation $\omega_{0}=0.0,0.3,0.6,0.9,1.2$. The time is given in units of initial half-mass relaxation time $\left(t_{\mathrm{rhi}}\right) \ldots \ldots \ldots$

4.11 Evolution of cluster radii for the model B4 $\left(0.6,0.9,5 \cdot 10^{-5}\right)$. Accretion, core, halfmass and tidal radii, are given in units of initial $r_{\mathrm{c}}$ are indicated in the legend. . . 
4.12 Evolution of mass shells (Lagrange radii $r_{55}$ ) for the models $W_{0}=6.0$ and initial rotation $\omega_{0}=0.0,0.3,0.9$. The percentage indicates the mass with respect to the total mass contained at the radii for mass columns in the direction of $\theta=54.74^{\circ}$. The time is given in units of initial half-mass relaxation time $\left(t_{\mathrm{rhi}}\right) \ldots \ldots \ldots$

4.13 Evolution of mass shells (Lagrange radii $r_{55}$ ) for the models $W_{0}=3.0$ and initial rotation $\omega_{0}=0.0,0.9,1.2$. The percentage indicates the mass with respect to the total mass contained at the radii for mass columns in the direction of $\theta=54.74^{\circ}$. The time is given in units of initial half-mass relaxation time $\left(t_{\mathrm{rhi}}\right) \ldots \ldots \ldots$

4.14 Evolution of mass shells (Lagrange radii $r_{55}$ ) for the models $W_{0}=9.0$ and initial rotation $\omega_{0}=0.2,0.4,0.5$. The percentage indicates the mass with respect to the total mass contained at the radii for mass columns in the direction of $\theta=54.74^{\circ}$. The time is given in units of initial half-mass relaxation time $\left(t_{\mathrm{rhi}}\right) . \ldots \ldots$

4.15 Evolution of $1 \mathrm{D}$ velocity dispersion with density, both evaluated at the $\mathrm{BH}$ influence radius for models of $W_{0}=6.0$ and initial rotation $\omega_{0}=0.0,0.3,0.6,0.9,1.2 \ldots$. .

4.16 Evolution of $1 \mathrm{D}$ velocity dispersion with density, both evaluated at the $\mathrm{BH}$ influence radius for models of $W_{0}=3.0$ and initial rotation $\omega_{0}=0.0,0.3,0.6,0.9,1.2 \ldots$. .

4.17 Evolution of $1 \mathrm{D}$ velocity dispersion with density, both evaluated at the $\mathrm{BH}$ influence radius for models of $W_{0}=9.0$ and initial rotation $\omega_{0}=0.2,0.3,0.4,0.5 \ldots$. . .

4.18 Evolution of $\mathrm{BH}$ and cluster mass for the model B3 $\left(6.0,0.6,5 \cdot 10^{-6}\right)$. The dotdashed line shows the mass of the cluster and the solid line the BH mass. Mass accretion rate is showed as a dashed line in units of $d\left(M_{\mathrm{BH}} / M_{\mathrm{cl}_{\mathrm{i}}}\right) / d\left(t / t_{\mathrm{rh}_{\mathrm{i}}}\right)$. The time is given in units of initial half-mass relaxation time $\left(t_{\mathrm{rhi}}\right) . \ldots \ldots \ldots$

4.19 Time evolution of cluster mass (green line) and BH-mass (black line). The red line shows the evolution of mass accretion rate $d M / d t$. Initial $M_{\mathrm{bh}}=5 \cdot 10^{-6} M_{\mathrm{cli}_{\mathrm{i}}}\left(25 M_{\odot}\right.$ for this model) and final $M_{b h} \simeq 0.01 M_{\mathrm{cl}_{\mathrm{i}}}\left(5000 M_{\odot}\right) \ldots \ldots \ldots \ldots$

4.20 Evolution of rotational velocity profile for the model B4 $\left(6.0,0.9,5 \cdot 10^{-6}\right)$. Note the formation of a maxima of rotation in the central region. Time is given parenthesis, in units of initial half-mass relaxation time $\left(t_{\mathrm{rhi}}\right) \ldots \ldots \ldots \ldots$

$4.212 \mathrm{D}$ rotational velocity distribution in the meridional plane for a model B4 $(6.0,0.9,5$. $\left.10^{-6}\right)$. Note the formation of a maxima of rotation in the central region. Time is given in units of initial half-mass relaxation time $\left(t_{\text {rhi }}\right) . \ldots \ldots \ldots$

4.22 Evolution of angular velocity with density, both evaluated at the $\mathrm{BH}$ influence radius for models of $W_{0}=6.0$ and initial rotation $\omega_{0}=0.3,0.6,0.9,1.2 \ldots \ldots$.

4.23 Evolution of angular velocity with density, both evaluated at the $\mathrm{BH}$ influence radius for models of $W_{0}=3.0$ and initial rotation $\omega_{0}=0.3,0.6,0.9,1.2 \ldots \ldots$. . .

4.24 Evolution of angular velocity with density, both evaluated at the $\mathrm{BH}$ influence radius for models of $W_{0}=9.0$ and initial rotation $\omega_{0}=0.2,0.3,0.4,0.5 \ldots \ldots$. . .

4.25 Evolution of rotational energy in units of total cluster energy for $W_{0}=0.6$. The epochs of maximum collapse are indicated by arrows. Time is given in units of initial half-mass relaxation time $\left(t_{\text {rhi }}\right) \ldots \ldots \ldots \ldots \ldots$

4.26 Evolution of rotational energy in units of total cluster energy for $W_{0}=0.3$. The epochs of maximum collapse are indicated by arrows. Time is given in units of initial half-mass relaxation time $\left(t_{\text {rhi }}\right) \ldots \ldots \ldots \ldots \ldots$

4.27 Evolution of rotational energy in units of total cluster energy for $W_{0}=0.9$. The epochs of maximum collapse are indicated by arrows. Time is given in units of initial half-mass relaxation time $\left(t_{\text {rhi }}\right) \ldots \ldots \ldots \ldots \ldots$ 
$4.28 V_{\text {rot }} / \sigma$ as a function of radius in units of initial core radius for the model B3 $\left(6.0,0.6,5 \cdot 10^{-6}\right)$ at collapse time. The dotted-dashed line represents the initial profile. The location of the initial maximum $V_{\text {rot }} / \sigma$ moves inward and drops in time. A second maximum grows in the core and in opposite direction of initial rotation . . . . . . . . . . . . . . . . . .

4.29 Evolution of angular velocity in cylindrical shells containing the masses indicated in the figure for the model B4 $\left(6.0,9.0,5 \cdot 10^{-6}\right)$. Time is given in units of initial $t_{\mathrm{rh}}$.

4.30 Evolution of specific angular momentum in cylindrical shells containing the masses indicated in the figure for the model B4 $\left(6.0,9.0,5 \cdot 10^{-6}\right)$. Time is given in units of initial $t_{\mathrm{rh}} \ldots \ldots \ldots \ldots \ldots \ldots \ldots \ldots \ldots$

4.31 Evolution of angular momentum averaged distribution function for a cluster with a final $M_{\mathrm{bh}} \simeq 0.01 M_{\mathrm{cl}_{1}}\left(\simeq 5000 M_{\odot}\right)$. The dashed-line shows the $1 / 4$-slope of the equilibrium solution. The initial and final profiles are shown through solid curves (the final state is the broader one), the dot-dashed curves show the evolution of the profiles. $E_{\text {crit }} \simeq 0.9$ in the last curve, at $t / t_{\mathrm{rh}_{\mathrm{i}}} \sim 3.5 \ldots \ldots \ldots \ldots$

4.322 Dimensional distribution function in $\left(E, J_{\mathrm{z}}\right)$-plane. Shown are the curves of constant energy (values listed in the left columns). The figure is separated in two panels, to make clear the $J_{\mathrm{z}}$-dependence of the distribution function specially at higher energies, due to loss-cone accretion of stars in low- $J_{\mathrm{z}}$ orbits. $E_{\text {crit }} \simeq 0.9$ and the time is the collapse time of the model $\mathrm{B} 4, t_{\mathrm{cc}} / t_{\mathrm{rh}_{\mathrm{i}}} \sim 3.5$. Isotropy would require a constant $f$, for all $J_{\mathrm{z}} \ldots \ldots \ldots \ldots \ldots \ldots \ldots \ldots$

4.33 Evolution of distribution function in the $E-Y$ plane for the model B5 $(6.0,1.2,5$. $\left.10^{-6}\right)$. The time is given in units of initial half-mass relaxation time $\left(t_{\mathrm{rhi}}\right) . \ldots$

4.34 Evolution of distribution function in the $E-Y$ plane for the model A5 (3.0,1.2, 5 . $\left.10^{-6}\right)$. The time is given in units of initial half-mass relaxation time $\left(t_{\mathrm{rhi}}\right) . \ldots \ldots$

4.35 Evolution of distribution function in the $E-Y$ plane for the model C3 $(9.0,0.4,5$. $\left.10^{-6}\right)$. The time is given in units of initial half-mass relaxation time $\left(t_{\mathrm{rhi}}\right) . \ldots$

4.36 Evolution of dynamical ellipticity for $W_{0}=0.3$. The epochs of maximum collapse are indicated by arrows. Time is given in units of initial half-mass relaxation time $\left(t_{\mathrm{rhi}}\right)$.

4.37 Evolution of dynamical ellipticity for $W_{0}=0.6$. The epochs of maximum collapse are indicated by arrows. Time is given in units of initial half-mass relaxation time $\left(t_{\mathrm{rhi}}\right)$.

4.38 Contour map of the rotational velocity and the total velocity dispersion (panels a and $b$ ) in the meridional plane, as can be obtained by observations, for a collapsed model B4 $\left(6.0,0.9,5 \cdot 10^{-6}\right)$. The bottom panels (c and d) show a zoom of the central parts of the cluster. Observe the increase of central rotation in torus-like contours of $v_{\text {rot }}$ and the second maxima formed in central regions close to the $\mathrm{BH}$. $\sigma_{t}$ shows higher contour levels in the center. Velocities are given in $\mathrm{km} / \mathrm{sec}$ and radii in parsec. The $\mathrm{BH}$ mass fraction over the initial cluster mass is $M_{\mathrm{bh}} / M_{\mathrm{cl}} \sim 0.01$ at $t_{\mathrm{cc}} / t_{\mathrm{rh}_{\mathrm{i}}} \sim 3.5 \ldots \ldots \ldots \ldots \ldots \ldots \ldots \ldots \ldots \ldots \ldots \ldots \ldots \ldots \ldots \ldots \ldots \ldots$ 


\section{Chapter 1}

\section{Introduction}

Globular clusters (GCs) are gravitationally bound concentrations of thousand to several million stars, spread over a volume of 10 to about 100 parsec in diameter. They are found in virtually every galaxy and host in general old stellar populations with ages of at least a few times $10^{9}$ yr (Gyr). Analysis of kinematic and photometric data of GCs have revealed the variety of environments in which these objects form and evolve (in distant halos or in the galaxy bulge). GCs show a wide range in central density, leading to interesting dynamical, star formation and stellar evolution phenomena. Their cores are unique laboratories for some fundamental dynamical processes, like two-body relaxation, angular-momentum transport, mass segregation, stellar collisions, stellar mergers, core collapse and stellar accretion by a central black hole, which occur on (local) time scales much shorter than the age of the universe. During the last years GCs have been used to obtain complete colourmagnitude-diagrams (CMDs) of simple stellar populations, which are the basis for testing stellar evolution on a star-by-star basis with the Hubble Space Telescope (HST) (Rich et al. 2005, see Fig. 1.2).

On the other side, distribution and properties of Globular Cluster Systems (GCS) provide a wealth of information on the formation and evolution of their parent galaxies. The discovery of the 'Fundamental Plane' * for elliptical galaxies (c.f. Djorgovski \& Santiago 1992; Djorgovski \& Meylan 1994 for reviews and references) has been extended in form of bivariate correlations for GCs of their core and half light properties (Djorgovski, 1995; McLaughlin, 2000). It represents an interesting constraint on the physics of GCs evolution. Radial distribution of GCSs follows a surface density profile $\Sigma_{\mathrm{GCS}} \propto r^{-\alpha}$, with $\alpha \approx 1.0-2.5$ (Ashman \& Zepf, 1998), while brighter galaxies show a more extended GC surface density. Most of them have been observed in giant elliptical galaxies and in the Local Group, as they host rich GCSs. The difficulty in the determination of the profile are the central galaxy regions, where the system is hidden in the noise of the high surface brightness of the host galaxy. On the other side, luminosity functions of GCSs can be well approximated by a Gaussian (Hanes 1977). The mean magnitude of the Milky Way and M31 is $M_{\mathrm{V}}=-7.4 \pm 0.2$ with a dispersion of $\sigma=1.2 \mathrm{mag}$ (Ashman \& Zepf, 1998), while HST observations in ellipticals, lead to a dispersion of $\sigma=1.4 \mathrm{mag}$ (Harris 2001). Relating density and luminosity distributions, the calculation of GCs number seems to correlate with galaxy luminosity, varying from a few in dwarf galaxies to a few ten thousand in giant elliptical

${ }^{*}$ The Fundamental Plane represents a set of bivariate correlations (luminosity or mass and surface brightness or radius), which provide hints for and constraints on the model of formation of elliptical galaxies 
galaxies (Kissler-Patig et al., 1997; Ashman \& Zepf, 1998).

The total number of clusters $N_{\mathrm{t}}$ is the simplest statistic describing a GCS. Hanes (1977) and Harris \& Racine (1979) demonstrated to first order that for a given type of galaxy, $N_{\mathrm{t}}$ scaled directly with galaxy luminosity. The specific frequency is defined as the cluster population normalized to $M_{\mathrm{V}}^{\mathrm{T}}=-15$,

$$
S_{\mathrm{N}}=N_{\mathrm{t}} \times 10^{-0.4\left(M_{\mathrm{V}}^{\mathrm{T}}+15\right)}
$$

showing remarkable first-order uniformity from galaxy to galaxy in the number of globular clusters per unit halo (spheroidal-component) luminosity over a vast range of sizes. An average $S_{\mathrm{N}} \simeq 4$ corresponds to one cluster per $M_{\mathrm{V}}$ (halo) $=-13.5$, or in terms of mass, of order $1 M_{\odot}$ in globular clusters per $\sim 10^{3} M_{\odot}$ in field-star mass. The specific frequency was introduced as a way to remove the first-order proportionality of $N_{\mathrm{t}}$ to galaxy size and thus to compare systems more easily. It turned out also to be an interesting discriminator for ideas about the formation of $\mathrm{E}$ galaxies by mergers. As a ratio of clusters to field halo stars, $S_{N}$ is relatively invariant to interactions between galaxies, because both stars and clusters alike behave essentially as massless test particles. Thus in a collision between two galaxies, $S_{\mathrm{N}}$ for the merged product will be a luminosity-weighted average between the two (or somewhat smaller if the remnant is stripped of gas and the age-dimmed disk light eventually joins the spheroid). The typical $S_{\mathrm{N}}$ for $\mathrm{Sa} / \mathrm{Sb}$ galaxies is similar to $S_{\mathrm{N}}$ for E galaxies in smaller groups, but significantly smaller than in the Virgo Es or the dwarfs. On this basis, Harris et al. (1985) suggested that spirals were fundamentally less efficient at forming globular clusters than were the Virgo ellipticals, and that the lower specific frequencies for field and small-group ellipticals might be explained by their higher expected merger rates; that is, many of the large ellipticals with the lowest SN values might indeed be remnants of long-past disk mergers.

Perhaps the most outstanding cosmological relevance of GCs their old age (e.g. Gratton et al. 1997). They come close to one stellar population, composed of stars with the same age and chemical composition and follows a passive, so-called pure luminosity evolution, after an initial, compared to their ages relatively short star-formation burst in which the cluster was formed. As photometric colour distributions of GC depend on metallicity and age, the existence of different colour modes leads to sub-populations of GCs. Statistical analyses reveal a bi-modal distribution in the shapes of, at least, half of the colour distributions in elliptical galaxies (Gerbhardt \& Kissler-Pattig, 1999; Larsen et al. 2001), as well as in M31 and the Milky Way and in a few GCSs in spirals outside the Local Group. From studies in the Local Group, was found that GC colour distributions are almost entirely driven by metallicity, although age effects can not be completely neglected in systems outside the Local Group. Galactic GCs are older than 10 Gyr, with a mean of 13 Gyr and a dispersion of 2-3 Gyr (e.g. Krauss \& Chaboyer, 2003), which makes them the oldest stellar structures in the Universe.

Kinematical studies of radial velocities of GCs can be used as tracer particles of the galaxy potential, as they are interestingly distributed in regions outside the limit of galaxy light, in the halo. For example, a net rotation found in the GC system in the Virgo giant ellipticals M87 (e.g. Cohen 2000) and NGC 4472 (e.g. Zepf et al.,2000) beyond $\sim 3 r_{\text {eff }}$ gives 
interesting insights on galaxy formation. On the other side, an increased velocity dispersion measured at large radii in the central giant elliptical NGC 1399 in the Fornax cluster, suggests that the outer GC population of this GCS traces the entire galaxy cluster potential.

Globular clusters are good candidates for being the most primeval, first forming structures which keep a fossil record of the earliest stages of galaxy formation (e.g. Searle \& Zinn 1978). Their ages submit to clocking galaxy formation and their chemical compositions mirror the element mix in the gas clouds out of which the first structures formed. This information holds important clues to global star formation and chemical enrichment, and therefore, to structure formation itself.

\section{$1.1 \quad$ Globular cluster parameters}

Globular clusters differ from other stellar systems mainly through their time scales of evolution, which follow

$$
t_{\mathrm{cl}}>t_{\mathrm{rh}} \gg t_{\mathrm{dyn}}
$$

where $t_{\mathrm{cl}}$ represents the cluster age, $t_{\mathrm{rh}}$ the half-mass relaxation time and $t_{\mathrm{dyn}}$ the dynamical time scale. The local relaxation time is defined as (Spitzer \& Hart, 1971)

$$
\tau_{\mathrm{rx}}=\frac{0.338 \sigma^{3}}{n(G m)^{2} \ln \Delta}
$$

where $\sigma$ is the one-dimensional velocity dispersion, $n$ the density, $G$ the gravitational constant, $m$ the mean stellar mass and $\ln \Delta$ is the Coulomb logarithm ( $\Delta=0.4 N$ is used here). At this time scale, a star experiences a mean change in velocity through gravitational scattering with other stars, which is comparable to the absolute value of its velocity at the beginning. Using this definition, the half-mass relaxation time, is defined as

$$
\tau_{\mathrm{rh}}=\frac{0.138 \sqrt{N r_{\mathrm{h}}^{3}}}{\sqrt{G m} \ln \Delta}
$$

$N$ is the number of particles (stars) and $r_{\mathrm{h}}$ the half-mass radius. $t_{\mathrm{rh}}$ represents the relaxation time of a sphere with homogeneous density, containing half of the total cluster mass at radius $r_{\mathrm{h}}$. The dynamical time is given by

$$
\tau_{\text {dyn }}=1.58 \sqrt{\frac{r_{\mathrm{h}}^{3}}{G M}}
$$

where $M$ is the total mass of the cluster. In this time, the cluster achieves a slowly evolution through diffusion in a sequence of virtual-equilibrium states. After a time $\tau_{\mathrm{rh}}$, information about the initial configuration is lost due to relaxation. It follows

$$
\tau_{\mathrm{rh}}=\frac{N}{11 \ln \Delta} \tau_{\mathrm{dyn}}
$$

For globular clusters, is $\tau_{\mathrm{rh}} \approx 10^{2}-10^{3} \tau_{\mathrm{dyn}}$. In the case of open star clusters, where the particle number varies between $N \simeq 100-500$, both timescales are comparable, and the 
evolution of equilibrium states can not be followed as previously discussed. The large number of stars in galaxies leads to a $\tau_{\text {rh }}$ larger than the age of the galaxy, and consequently, time is not enough to achieve a dynamical state of equilibrium.

Characteristic radii are frequently used in the description of evolution in GCs. The core radius is defined from observations, as the radius, at which the cluster surface brightness is half its central value. Theoretical models based on a lowered Maxwellian distribution, generally called King models (King, 1966), give following definition of this radius

$$
r_{\mathrm{c}}=\left(\frac{3 \sigma_{\mathrm{c}}^{2}}{4 \pi G n_{\mathrm{c}}}\right)^{1 / 2}
$$

where $\sigma_{\mathrm{c}}$ and $n_{\mathrm{c}}$ are the central velocity dispersion and density, respectively.

As the present study concentrate on evolution of clusters with black holes (BHs), following characteristic radii are of interest. The disruption radius, within which a star would be disrupted by a $\mathrm{BH}$ in its vicinity, is given for a solar type star of mass $m_{*}$ and radius $r_{*}$, by (Frank \& Rees, 1976)

$$
r_{\mathrm{d}} \propto r_{*}\left(M_{\mathrm{bh}} / m_{*}\right)^{1 / 3}
$$

Frank \& Rees (1976) also defined an important radius in the vicinity of BHs, where its potential well affects the stellar velocity field of the system

$$
r_{\mathrm{a}}=\frac{G M_{\mathrm{BH}}}{\sigma_{\mathrm{c}}^{2}}
$$

where $M_{\mathrm{BH}}$ is the mass of the $\mathrm{BH}$.

Observational parameters of representative high elliptical galaxy GCs are shown in Table (1.1), with the exception of those without ellipticity, M80 and M4. The ellipticity $e=1-(b / a)$, with the semimajor and semiminor axis of the cluster light profile $a$ and $b$, respectively. Characteristic radii are core radius, half-mass radius (radius which contains half of the cluster mass), tidal radius $\left(r_{\mathrm{t}}\right)$ where the density of the cluster drops to zero and the concentration parameter $c=\ln \left(r_{\mathrm{t}} / r \mathrm{c}\right)$, which is typically high in galactic GCs, as they are generally evolved systems. The data was taken from the Harris Catalog of Milky Way Globular Clusters (Harris et al., 1996, February 2003 revision). The relaxation time at the half-mass radius was calculated using a mean mass to light ratio of $M / L=2$. Cluster ages were taken from the literature (see references in the table caption). Note that the listed values of $c$ and $r_{\mathrm{c}}$ of M15 should not be used to calculate the tidal radius $r_{\mathrm{t}}$ for this core-collapsed cluster as explained in the catalog.

\subsection{Globular clusters in the Local Group}

Basically all galaxies in the Local Group (with the exception of M32) host GCSs, and the same can be expected for virtually all galaxies outside the Local Group. Observational study of GCs in the Local Group is straightforward due to their proximity, while its quality 


\begin{tabular}{|r|r|r|r|r|r|r|r|}
\hline & $r_{\mathrm{c}}$ & $r_{\mathrm{h}}$ & $r_{\mathrm{t}}$ & $c$ & $\mathrm{e}$ & $t_{\mathrm{rh}}[\mathrm{yr}]$ & age [yr] \\
\hline \hline NGC 104 (47Tuc) & 0.40 & 2.79 & 42.86 & 2.03 & 0.09 & $3.01 \cdot 10^{9}$ & $1.2 \pm 0.1 \cdot 10^{10}(4)$ \\
\hline NGC 2808 & 0.26 & 0.76 & 15.55 & 1.77 & 0.12 & $1.30 \cdot 10^{9}$ & $1.6 \pm 2.0 \cdot 10^{10}(1)$ \\
\hline NGC 5139 (wCen) & 2.58 & 4.80 & 44.83 & 1.24 & 0.12 & $1.00 \cdot 10^{10}$ & $1.6 \pm 0.3 \cdot 10^{10}(7)$ \\
\hline NGC 5286 & 0.29 & 0.69 & 8.36 & 1.46 & 0.12 & $1.0 \cdot 10^{9}$ & $1.6 \pm 0.2 \cdot 10^{10}(5)$ \\
\hline NGC 5904 (M5) & 0.42 & 2.11 & 28.40 & 1.83 & 0.14 & $3.38 \cdot 10^{9}$ & $13.5 \pm 1.0 \cdot 10^{9}(6)$ \\
\hline NGC 6093 (M80) & 0.15 & 0.65 & 13.28 & 1.95 & 0.00 & $7.20 \cdot 10^{8}$ & $1.40 \cdot 10^{10}(2)$ \\
\hline NGC 6121 (M4) & 0.83 & 3.65 & 32.49 & 1.59 & 0.00 & $6.60 \cdot 10^{8}$ & $13.5 \pm 1.0 \cdot 10^{9}(6)$ \\
\hline NGC7078 (M15) & 0.07 & 1.06 & 21.50 & 2.50 & 0.05 & $2.23 \cdot 10^{9}$ & $13.0 \pm 3.0 \cdot 10^{9}(3)$ \\
\hline \hline
\end{tabular}

Table 1.1: Column 1: Cluster identification number; Column 2: $r_{\mathrm{c}}$, current core radius in arcminutes; Column 3: $r_{\mathrm{h}}$, current half mass radius in arc-minutes; Column 4: $r_{\mathrm{t}}$, current tidal radius in arc-minutes; Column 5: $c \equiv \log _{10}\left(\mathrm{r}_{\mathrm{t}} / \mathrm{r}_{\mathrm{c}}\right)$, concentration; Column 6: e, ellipticity (projected axial ratio); Column 7: $t_{\mathrm{rh}}$, half mass relaxation time in years; Column 8: cluster age in years taken from the literature, using following references:

(1) Alcaino et al. (1990);(2) Brocatto et al., 1998; (3) Caputo et al., 1984; (4) Grundahl et al., 2002; (5) Samus et al., 1995; (6) Sandquist et al., 1996; (7) Kinematical data from Trager et al., 1995, age from Thompson et al., 2001 
has been considerably improved in the last decades.

The Milky Way (MW) possesses $160 \pm 20$ globular clusters (Harris 1996, February 2003 revision), which have proved invaluable for understanding its structure, stellar populations, and mass accretion history (e.g., Searle \& Zinn 1978). They are composed of at least two components: a spatially extended, metal-poor, pressure-supported system and a metalrich, centrally concentrated system that exhibits some rotation (Zinn 1985). The majority of these metal-rich clusters are now believed to be associated with the bulge (Frenk \& White 1982; Minitti 1995; Coté 1999; Forbes et al. 2001). Distributions and correlations of global properties of galactic GCs have been studied extensively in an effort to provide the global picture of their formation and evolution (Chernoff \& Djorgovski 1989; Djorgovski \& Meylan 1994; Djorgovski 1996; Bellazzini 1998). The 'basic' cluster properties are considered as being the total luminosity (mass), binding energy, mass-to-light ratio, concentration, core radius and velocity dispersion (Kormendy 1985; Djorgovski 1995 ; McLaughlin 2000). Fig. (1.1) shows the positions of the roughly spherical distribution of globular clusters around the center of the Milky Way (Harris 1996, February 2003 revision), marked as gray dots on top of the COBE infrared map of the Galaxy. The six enlargements are taken from Table (1.1) as representative elliptical GCs.

GCs in the MW range among the oldest stellar structures in the universe and the same was long assumed for their extragalactic analogues. The hypothesis that relatively young GCs could form in galaxy-galaxy collisions (Schweizer, 1987) revised the picture of ancient stellar systems and lead to a quantitative prediction of the properties of GCSs which formed in galaxy mergers (Ashman \& Zepf, 1992). The confirmation of some of those predictions, such as bimodal GCs colour distributions, by Zepf \& Ashman (1993) marked a turning point in GCs research. In subsequent years, GCs formation was observed in many merging galaxies and merger remnants. Photometric and the still sparse spectroscopic observational evidence until the recent past is described in detail in Ashman \& Zepf (1998) and suggests that GCs form ubiquitously during star-formation events which build up galaxies.

M31 has the largest population of globular clusters ( $460 \pm 70$; see Barmby \& Huchra 2001). Since M31 and the MW are quite similar by being normal disk galaxies, the M31 globular-cluster system is particularly valuable for comparison with Milky Way GCs. Nevertheless, there are also important differences. Brown et al. (2003) found evidence for an age dispersion in the halo, with a metal-rich population as young as 6-8 Gyr old. The halo itself appears to be dominated by stars acquired from the ingestion of other stellar systems (Ferguson et al. 2002). Correlations of cluster properties have been intensively analyzed (Djorgovski et al. 1997; Dubath \& Grillmair 1997). Barmby et al. (2002) found that ellipticities of M31 GCs are consistent with being caused by rotation. At the distances of M31 (and M33) the HST has greatly improved observations (Rich et al. 2005, see Fig. 1.2). One pixel in the Planetary Camera (PC) corresponds to about $0.2 \mathrm{pc}$ in comparison with a typical ground-based resolution of $\sim 1^{\prime \prime}$, corresponding to a linear scale of about $4 \mathrm{pc}$. This is of great importance not only for studies aiming at a morphological characterization of the clusters, but also for dynamical studies in which velocity dispersions must be tied to knowledge about the cluster structure in order to provide accurate mass-to-light ratios. Trudolyubov \& Priedhorsky (2004) detected 43 X-ray sources in M31, based on the data of XMM-Newton and Chandra observations, coincident with GC candidates from various optical surveys. They suggest the M31 GC X-ray sources to be low-mass X-ray binaries 


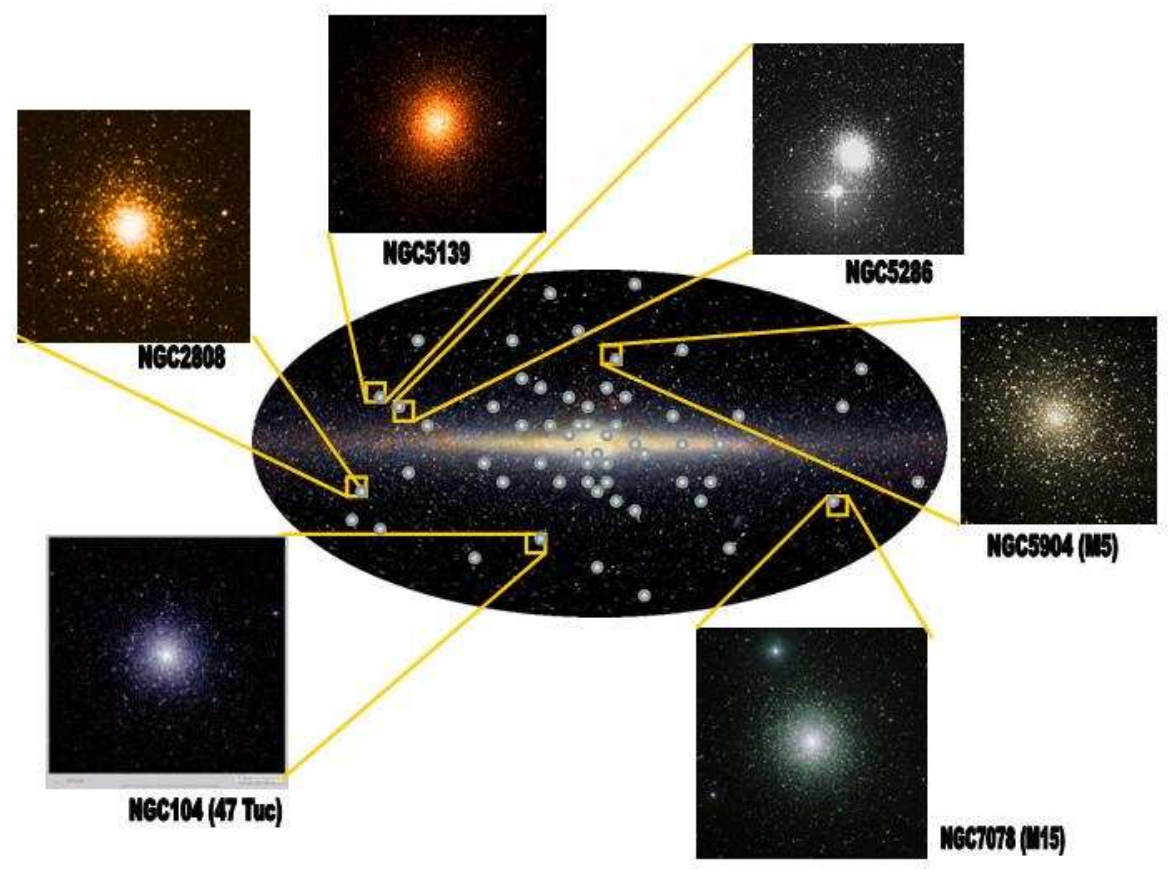

Figure 1.1: Distribution of globular clusters in the Milky Way. Some clusters of Table (1.1) are illustrated. M5, $\omega$ Cen and 47 Tuc are at distances between $6-7 \mathrm{kpc}$; NGC 5286 is at $8.4 \mathrm{kpc}$; M15 and NGC2808 are located at 10-11 kpc from the galactic center. The Milky Way picture was taken by the COBE satellite and shows the plane of our Galaxy in infrared light. The thin disk is clearly apparent, with stars appearing white and interstellar dust appearing red. It is overploted by globular cluster positions taken from the Harris Catalog (Harris et al., 1996, February 2003 revision). 
(LMXB) systems with neutron star primaries. However, the current X-ray spectral and timing data cannot rule out the possibility of finding active accreting black holes in the GC sample.
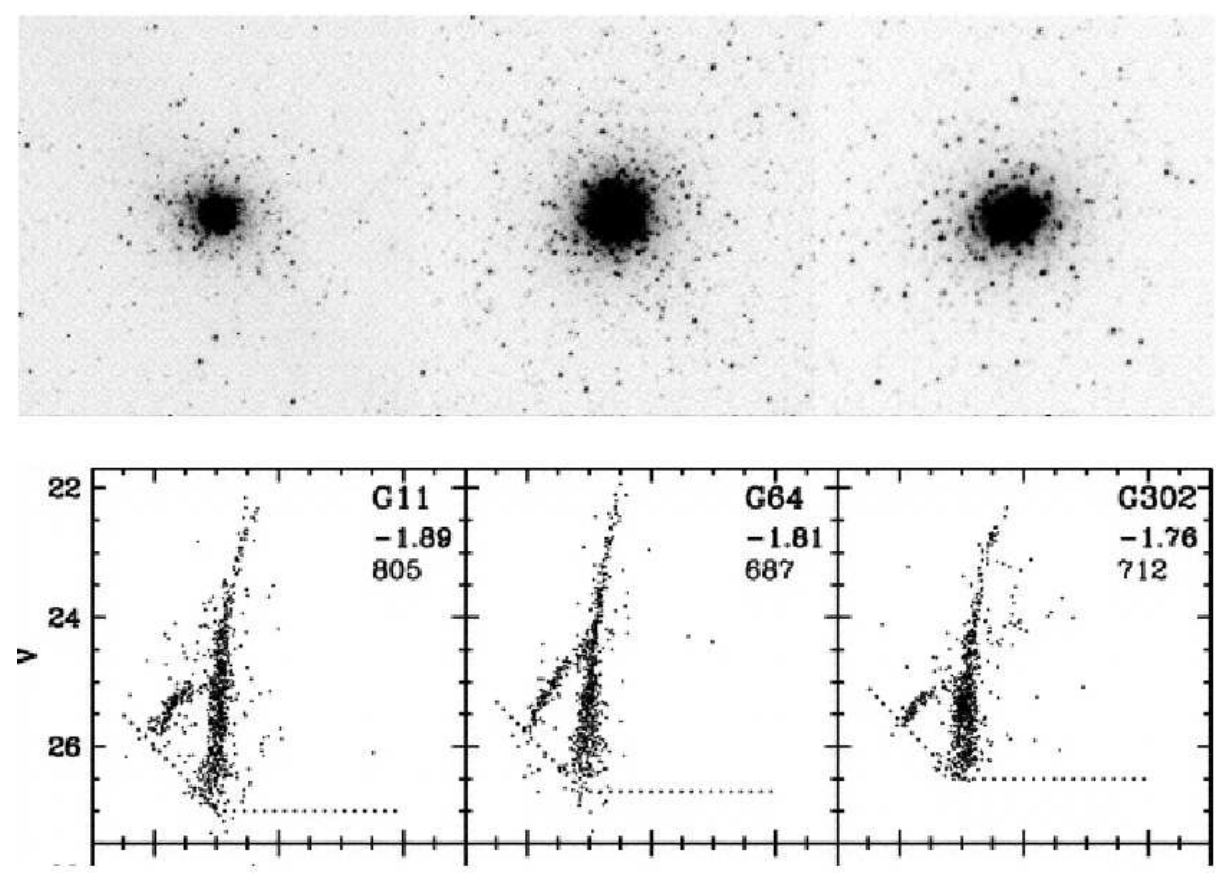

Figure 1.2: HST images of M31 GCs (G11, G64 and G302) analyzed in Rich et al. 2005. All are $20^{\prime \prime} \times 20^{\prime \prime}(=80 \mathrm{pc})$ sub-rasters (upper figures). The corresponding CMDs are shown below. Each cluster is labeled with its name, metallicity, and number of stars displayed.

Studies of GCs associated with both the Large Magellanic Cloud (LMC) and the Small Magellanic Cloud (SMC) have an advantage over those of GCs in our Galaxy in that they span a wider range in ages, which allows study of stellar evolution of various masses. The system of rich star clusters in the LMC includes clusters of comparable mass to galactic GCs but which span the entire age range from less than $5 \odot 10^{6} \mathrm{yr}$ (e.g.R136) to about $10^{10}$ yr (e.g. NGC 2019). This allows the observation of massive star clusters at various epochs in their evolution and makes it possible to identify evolutionary trends (if external processes affecting cluster evolution have not changed significantly over the lifetime of the LMC). One of this is the reported by WIlkinson et al. (2003), using a selection of 8 LMC clusters, which have similar masses and metallicities and lie at comparable radii from the LMC center but differ significantly in other properties such as core radius. Their age range from about $10^{7}$ yr up to $10^{10} \mathrm{yr}$. The youngest clusters (NGC 1805 and NGC 1818) are old enough to have expelled all their residual gas and so are pure N-body systems. This correlation suggests some evolutionary behavior, but the processes investigated so far (binary activity, variations of the IMF, variations of the tidal field) cannot sufficiently reproduce the observed scatter of radii with age (de Grijs et al. 2002b; Mackey \& Gilmore 2003; Wilkinson et al. 2003). The star clusters of the LMC represent a unique opportunity of studying young stellar populations and they are close enough to allow detailed observation of single stars. Old as well as young and rich LMC clusters help us to study several effects related to gravitational dynamics of $N$-body systems, including mass segregation and stellar evaporation 
(Santiago et al. 2001; de Grijs et al. 2002a). These effects are an important step towards recovering the cluster initial conditions and assessing the universality of the initial mass function (Goodwin 1997; Paresce \& de Marchi 2000; Kroupa et al. 2001; Kroupa 2002; de Grijs et al. 2002b).

The formation of GCs is tightly linked to the star-formation history of galaxies and thus, can be used as probes of galaxy formation. They are presumably formed in giant molecular clouds (Fall \& Rees 1985; Elmegreen \& Efremov 1997). The cloud becomes unstable as the Jeans mass is exceeded, and it begins to collapse. Fragmentation of the gas sets on, and clumps are formed which subsequently turn into stars in the densest core regions of the cloud. Apart of it it gas expulsion takes place, driven by radiative feedback of young massive stars, as well as stellar winds and supernova explosions, as the star formation terminates. Due to the expulsion of the remaining gas, the cluster can dissolve on dynamical timescales or survive as a dynamically stable system only if less than $50 \%$ of the gas is expelled and the rest transformed into stars (Hills 1980).

Thus, GCs can form anytime where enough gas can be cooled and/or be compressed. During their stable phase, giant molecular clouds are believed to be supported by supersonic turbulence (Harris \& Pudritz 1994) until an outside trigger initiates the collapse. However, to cool efficiently the presence of metals is required. Some GCs have metallicities which are too low to allow efficient cooling of the parent cloud, and therefore imply different ways to initiate the collapse of metal-poor primordial gas clouds. For instance, gravitational stress can increase the ambient gas pressure and induce the collapse by pressure instabilities (Elmegreen \& Efremov 1997). Another trigger mechanism can be convergent shocks caused by the external radiation field during the re-ionization epoch (Cen 2001). Moreover, star clusters are very likely to form in giant molecular clouds, and survive a Hubble time as dynamically stable systems.

\subsection{Flattening and rotation}

Many globular clusters do show some amount of rotation, even the old ones of our own galaxy (Meylan \& Mayor 1986; Lupton, Gunn \& Griffin 1987; Gebhardt K., personal communication). The more accurate star-by-star observations become available, the more important it is to properly model the effects of rotation together with evolution of globular star clusters, since typically the amount of rotational energy in clusters is not dominant, but also not negligible. It is long known that indeed flattening of galactic globular clusters correlates with their rotation, suggesting that rotation still is important for the shape (White \& Shawl 1987). Proper Motions of stars in $\omega$ Cen have been measured to get a real 3D profile of stellar motions including rotation, and in observations using the Hubble Space Telescope it became even possible to measure rotation of 47 Tuc in the plane of the sky (Anderson \& King 2003). While without rotation the wealth of observational data such as luminosity functions and derived mass functions, CMDs, and population and kinematical analysis, obtained by e.g. the Hubble Space Telescope, for extragalactic as well as Milky Way clusters (cf. e.g. Piotto et al. 1999a,b; Rubenstein \& Bailyn 1997; Ibata et al. 1999; Grillmair et al. 1999; Shara et al. 1998), is balanced by a decent amount of detailed modelling, theorists and observers alike seem to be appallingly puzzled by rotation in clusters, and are disappointed that (multi-mass) King model fitting does not work very well (see e.g. Piotto 
et al. 1999b, and for a specific example Mc Laughlin et al. 2003, therein).

The observed flattening of GCs (Geyer et al. 1983; White \& Shawl 1987; van den Bergh 1996) can be caused by either anisotropy in the velocity dispersion or through rotation. Meylan \& Mayor (1986) confirmed the global rotation in clusters such as $\omega$ Cen and 47 Tuc. Direct measurements of rotational velocity $\left(V_{\text {rot }}\right)$ in GCs were carried out by measuring the radial velocity of a large number of cluster members (up to a few hundred). Gebhardt et al. (1994) and Gebhadrt \& Fischer (1995) reported radial velocity observations of several GCs including M15 and 47 Tuc. From projected rotational velocity curves, they estimated $V_{\text {rot }} / \sigma$ of each cluster, where $\sigma$ denotes the one-dimensional velocity dispersion. Both M15 and 47 Tuc have $V_{\text {rot }} / \sigma \simeq 0.2-0.4$ in the central region with the position angle of the rotational axis coinciding roughly with the semi-minor axis. Later, Gebhardt et al. (2000) found an increase in the rotation velocity of M15 toward the center of the cluster, with a maximum of $V_{\text {rot }} \approx 13 \mathrm{~km} \mathrm{~s}^{-1}$ at their innermost (trustworthy) radius $R \approx 0^{\prime \prime} .5$. The velocity dispersion $\sigma$ shows a mild inward decrease at small radii, from $\sigma \approx 14 \mathrm{~km} \mathrm{~s}^{-1}$ at $R \approx 4^{\prime \prime}$; to $\sigma \approx 10 \mathrm{~km} \mathrm{~s}^{-1}$ at $R \approx 0^{\prime \prime} .5$. Rotation HST measurements of 47 Tuc have recently confirmed earlier studies of rotational velocities in this GC (Anderson \& King 2003). Using a non-parametric technique on 469 radial velocity measurements Merritt et al. (1997) obtained two dimensional structures of rotational velocity in $\omega$ Cen. Van Leeuwen et al. (2000) found agreements of proper-motion studies with rotation in this cluster.

\begin{tabular}{|r|r|r|r|}
\hline & $v_{\text {rot }}^{\max }$ & $v_{\text {rot }} / \sigma$ & $e$ \\
\hline \hline NGC 104 (47Tuc) & 5.7 & 0.23 & 0.09 \\
\hline NGC 5139 $(\omega C e n)$ & 7.9 & 0.41 & 0.17 \\
\hline NGC7078 (M15) & 13.0 & 1.3 & $0.05(\mathrm{c})$ \\
\hline M22 & 3.8 & 0.5 & 0.14 \\
\hline M2 & 5.5 & 0.34 & 0.11 \\
\hline M92 & 2.5 & 0.3 & 0.10 \\
\hline NGC 3201 & 1.2 & 0.28 & 0.12 \\
\hline M13 & 5.0 & 0.25 & 0.11 \\
\hline M30 & 1.1 & 0.12 & $0.01(\mathrm{c})$ \\
\hline M3 & 1.0 & 0.12 & 0.04 \\
\hline NGC6397 & 0.5 & 0.11 & $0.07(\mathrm{c})$ \\
\hline NGC362 & 0.0 & 0.01 & $0.01(\mathrm{c})$ \\
\hline \hline
\end{tabular}

Table 1.2: Rotational parameters of globular clusters. Column 1: Cluster identification number; Column 2: $v_{\text {rot }}^{\max }$, maximum of rotational velocity in $\mathrm{km} \mathrm{s}^{-1}$; Column 3: $v_{\text {rot }} / \sigma$, relation of rotation over velocity dispersion; Column 4: e, cluster ellipticity. (c) denotes a core-collapsed cluster. Data is taken from Harris 1996, Heggie \& Meylan 1997 and recent literature.

In addition, significant radial variation of the ellipticity (Kontizas et al. 1990) and of the peak rotational velocity (Merritt et al. 1997), are closely related to the dynamics and evolution of GCs in interaction with galaxies. Han \& Ryden (1994) have argued that LMC star clusters are likely triaxial structures, while those in the Galaxy are spheroidal if not spherical. They applied a non-parametric fitting method (developed by D. Merritt, see e.g. Tremblay \& Merritt 1995) to surface brightness profiles of a number of clusters in the Galaxy, the LMC and M31, and conclude that younger clusters are systematically more a-spherical, confirming an earlier study of Frenk \& Fall (1982). Table (1.2) shows 
the available rotational parameters of galactic globular clusters. Some of them are also illustrated in Fig. (1.1). Note that, the position of the maximum of rotation in M15 is located very close to the cluster center (Gebhardt et al. 2000).

\subsection{Black holes in dense stellar systems}

Observational evidences in the last years (like HST measurements of nearby galaxies) suggest that massive BHs are present at the centre of most galaxies. Theoretical modelling of measured motions request for the presence of a central compact dark object with a mass of $\sim 10^{6}$ to $10^{9} M_{\odot}$ (Ferrarese et al., 2001; Gebhardt et al., 2002; Pinkney et al., 2003; Kormendy, 2003). Ground-based IR observations of the fast orbital motions of a few stars in the Milky Way have lead to the suggestion of a $3-4 \times 10^{6} M_{\odot} \mathrm{BH}$ in its center (Ghez et al., 2003; Schödel et al., 2003). Nowadays it is well accepted that kinematics in local active and non-active galaxies can be explained by the presence of a central MBH. Moreover, BH demographics have lead to correlations between the BH mass and the luminosity of its host bulge or elliptical galaxy (Kormendy \& Richstone, 1995), and between BH mass and the velocity dispersion of its host bulge, as $M_{\mathrm{BH}} \propto \sigma^{\alpha}$ (Ferrarese \& Merritt, 2000). This leads to a strong link between $\mathrm{BH}$ formation and the properties of the stellar bulge.

Swallow or distortion of high velocity stars, which achieve velocities up to $1000 \mathrm{~km} / \mathrm{sec}$, in the high density core $\left(\sim 10^{6} \mathrm{pc}^{-3}\right)$ can happen, under these conditions. Furthermore, gravitational scattering and real collisions occur, while stars can produce gas to be accreted on to the $\mathrm{MBH}$ through normal stellar evolution, collisions or disruptions of stars by the strong central tidal field. These processes lead to a strong increasing of the BH mass (Murphy et al., 1991; Freitag \& Benz, 2002). Tidal disruptions trigger phases of bright accretion that may reveal the presence of a $\mathrm{MBH}$ in very distant galaxies (Hills, 1975; Gezari et al., 2003). Stars are totally accreted if they approximate the horizon of the $\mathrm{BH}$ or are captured by emission of gravitational waves (GWs), one of the main targets of the project LISA (Laser Interferometer Space Antenna). In spherical systems in dynamical equilibrium, only collisional effects can bring stars on to the 'loss-cone', as high energy eccentric orbits come close to the BH. Nevertheless, non-spherical structures such as triaxial bulges, bars or stellar discs are common on scales of 100-1000 pc, and also the nucleus itself may be non-spherical. It could rotate, as a result of a merger with another nucleus (Milosavljevic \& Merrit, 2001) or due to dissipative interactions between the stars and a dense accretion disc (Rauch, 1995). The influence of non-sphericity at small and intermediate scales on the structure and evolution of the nucleus has not been well studied, and it could change the estimates of capture and disruption rates by orders of magnitudes (Merritt \& Poon, 2004).

On the other side, observational evidence for the existence of intermediate-mass black holes (IMBHs) in star clusters is as yet not very clear. Local core-collapse clusters are obvious places to look for IMBHs (high central densities and since they are relatively close). Gerssen et al. $(2002,2003)$ reported the kinematical study (based on HST spectra) of the central part of the collapsed GC M15, showing a steep increase in the radial profile of $V_{\text {rot }} / \sigma$

and a rapid decline followed by a slowly rising behavior. They proposed the presence of an IMBH $\left(M_{\mathrm{BH}}=3.9 \cdot 10^{3} M_{\odot}\right)$ in the central region of M15. A single or binary IMBH could account for the net rotation observed in the center of M15 (Gebhardt et al. 2000; Gerssen et al. 2002; Miller \& Colbert 2004). However, Baumgardt et al. (2003) have 


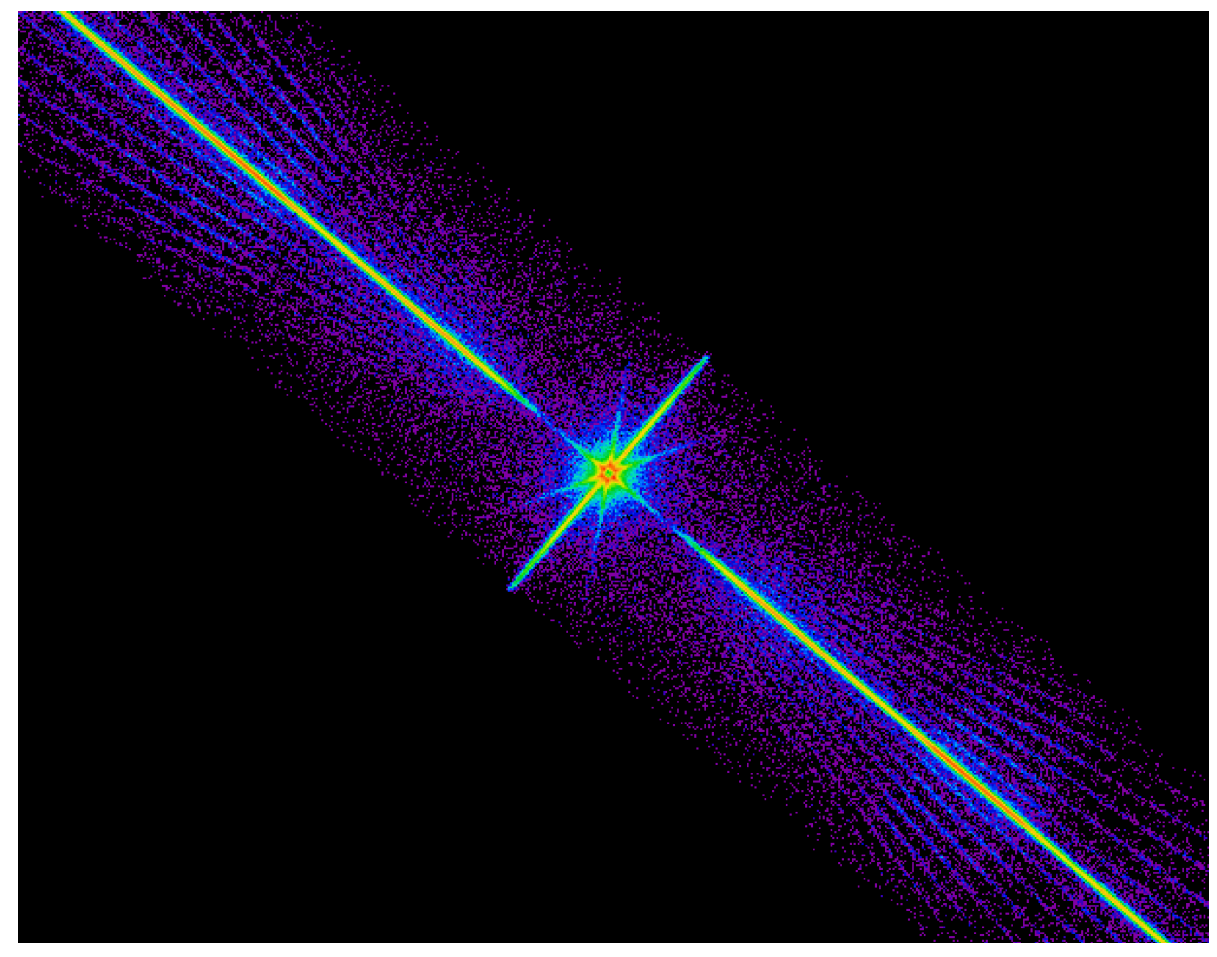

Figure 1.3: Chandra image of the stellar mass black hole candidate at the position of the quiescent X-ray nova XTE J1118+480 (central bright point). The spikes and rays extending from the bright point are instrumental artifacts. From McClintock et al. (2003). $M_{\mathrm{BH}}<10 M_{\odot}$.

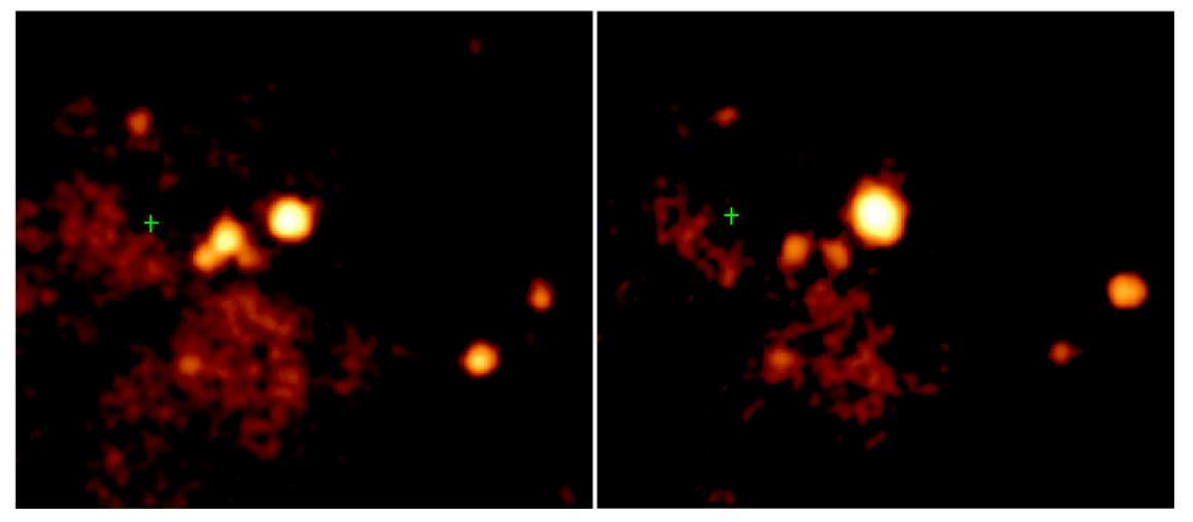

Figure 1.4: Chandra Image of the black hole discovered in a dense star cluster about 600 light years from the center of the galaxy M82. Astronomers found that the mass of the black hole must be greater than $500 M_{\odot}$. 


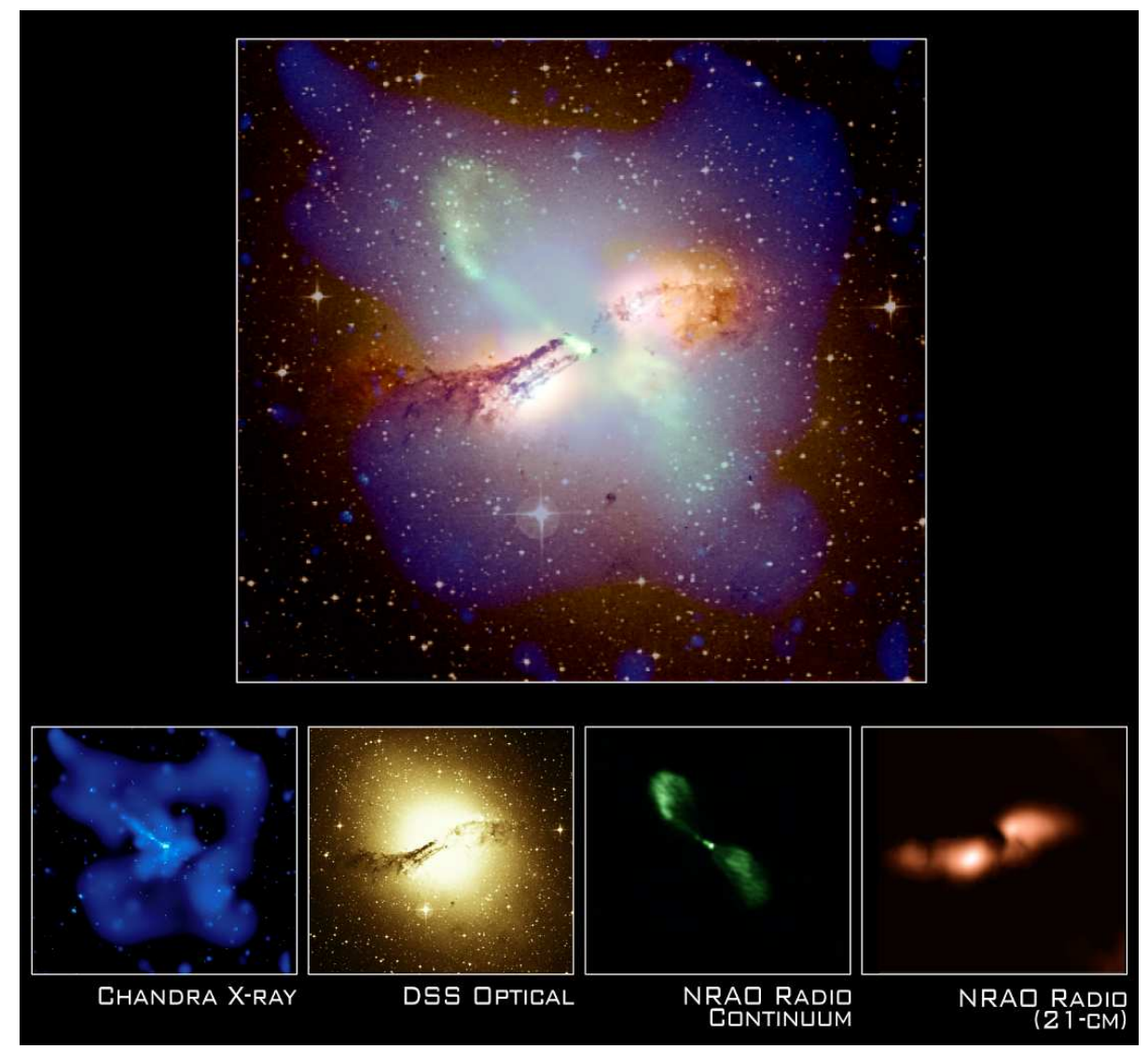

Figure 1.5: A composite X-ray (blue) from Karovska et al. (2002); radio (pink and green) from Van Gorkom et al. (1994), and optical (orange and yellow) from Digitized Sky Survey U.K. Schmidt Image/STScI; image of the galaxy Centaurus A. A broad band of dust and cold gas is bisected at an angle by opposing jets of high-energy particles blasting away from the supermassive black hole in the nucleus $\left(M_{\mathrm{BH}} \sim 1-3 \cdot 10^{8} M_{\odot}\right)$ 
shown through self-consistent $N$-body computations treating stellar evolution and with a realistic IMF, that the core-collapse profile of a star cluster with an unseen concentration of neutron stars and heavy-mass white dwarfs can explain the observed central rise of the mass-to-light ratio (see also McNamara et al. 2003). Similarly, a dense concentration of compact remnants might also be responsible for the high mass-to-light ratio of the central region of NGC 6752 seen in pulsar timings (Ferraro et al. 2003; Colpi et al. 2003). Outside our own galaxy, Gebhardt et al. (2002) have reported evidence for a $20000 M_{\odot} \mathrm{BH}$ in the M31 globular cluster G1.

Chandra and XMM-Newton observations of ultraluminous X-ray sources (ULXR) give also evidence for the existence of IMBHs in dense star clusters, which are often associated with young star clusters and whose high X-ray luminosities in many cases suggest a compact object mass of at least $10^{2} M_{\odot}$ (Ebisuzaki et al. 2001; Miller et al. 2003). Furthermore, some of the ULXR sources detected in other galaxies are may be accreting intermediatemass black holes (IMBHs) (e.g., Miller \& Colbert 2004), although the majority could be likely stellar-mass black holes (King et al. 2001; Rappaport et al. 2005).

The variety of masses of $\mathrm{BH}$ candidates detected in the last years is illustrated exemplarily in Figs. (1.3) to (1.5). A stellar mass black hole candidate $\left(M_{\mathrm{BH}}<10 M_{\odot}\right)$ at the position of the quiescent X-ray nova XTE J1118+480 is shown in Fig. (1.3). Fig. (1.4) shows a Chandra image of an intermediate-mass $\mathrm{BH}\left(M_{\mathrm{BH}}>500 M_{\odot}\right)$ discovered in a dense star cluster at about 600 ly from M82. In Fig. (1.5) a composite X-ray, radio and optical image of the massive elliptical galaxy M82 is presented, which harbors a supermassive BHs $\left(M_{\mathrm{BH}} \sim 1-3 \cdot 10^{8} M_{\odot}\right)$. M82 hosts a strong, variable X-ray/radio source and a massive, complex disk composed of dust, gas, and young stars.

Parallel to the observational efforts the theoretical path to globular cluster evolution is summarized in the next Chapter.

\subsection{Aims of this study}

The variety of environments in which GCs form and evolve, make them unique laboratories for some fundamental dynamical processes, like two-body relaxation, angular-momentum transport, mass segregation, stellar collisions, stellar mergers, core collapse and stellar accretion by a central black hole, which occur on (local) time scales much shorter than the age of the universe. Observational analysis has been considerably improved in the last years, leading to a better understanding of these evolutionary processes, but at the same time, opening new questions due to the, in the past unimpressive, complexity of this stellar systems. This is the reason, why theoretical models are of importance to elucidate the origin of the observed phenomena, and to be able to explain formation and evolution processes, that happen inside GCs. On the other side, theory is able to predict possible evolutionary scenarios, which can be confirmed by observations in the near future.

The present work contains theoretically formulated models in the evolution of globular star clusters, extending the model complexity with following elements. First, as GCs are not really spherical, flattening, originated by rotation, becomes an important target in theoretical models. There still not exist a wide set of models, which can explain all the 
evolutionary implications in the complexity of rotational evolution in GCs. Second, observational evidences of the existence of central dark objects in some GCs, with and without rotation, motivates the inclusion of this constraint in the long term cluster evolution. Thus, the present work accomplishes for the first time, a complete set of self-consistent evolutionary models of GCs with an embedded $\mathrm{BH}$ and system rotation. It takes advantage of an statistical method, in order to allow the computation of a large number of stellar components, and follow the system development in large times scales. With this study, theoretical modelling goes ahead of observations, since evolution of high resolution $2 \mathrm{D}$ distribution for densities, velocity dispersions and rotational velocities, deep in the stellar cusp surrounding the central BH is provided. All quantities are self-consistently coupled in an evolutionary model for the $\mathrm{BH}$ growth and rotating star cluster evolution.

The main theoretical questions aimed to be answered in this work, are related first to how the presence of a central dark object modifies, or not, the evolution of system rotation in self-gravitating stellar systems driven by relaxation processes; and second, at the same time, how rotation influences BH evolution (growing through stellar accretion). Existent models of rotating self-gravitating systems without $\mathrm{BH}$ and spherically symmetric $\mathrm{BH}$ models without rotation are useful to compare results and confirm, or not, final states of equilibrium in this kind of objects. The results obtained with this studie are mainly applicable to GCs and extensive to the dynamical evolution of other dense stellar systems, like galactic nuclei. AS mentioned, $\mathrm{BH}$ masses expected in the latter systems are higher than in GCs by many orders of magnitude, while their environments and nature of complexity of phenomena in this systems is a further astrophysical motivation.

After a general introduction of the problem and description of mainly observational knowledge of the elements taken into account, was made in the present chapter (Chapter1). Chapter 2 gives a description of the theoretical knowledge, so far, applied to the problem. The advantages and disadvantages of the different applicable methods are compared. In Chapter $\mathbf{3}$ the choice of the method used in the present study is justified. A detailed description of the method used in the present work is made, with the aim to follow the evolution of rotating self-gravitating star clusters with a central BH. An anisotropic statistical model that solves numerically the full Fokker-Planck equation with the Boltzmann-Vlasov term on the left- and an interaction (collisional) approximation on the right-hand side, is used. All quantities of interest (densities, dispersion, rotation) are accessible as smooth functions of the radius and time. Furthermore, a distribution in energy $(E)$ and angular momentum $\left(J_{\mathrm{z}}\right)$ coordinates of the number density of stars is computed, in order to facilitate the understanding of the evolutionary parameters in real space. A self-consistent method of $\mathrm{BH}$ star accretion driven through angular momentum diffusion, defined in $\left(E, J_{\mathrm{z}}\right)$-space, is described in this chapter. Chapter 4 presents the main results, concentrating first on the general cluster structure and evolution of $\mathrm{BH}$ through star accretion, and secondly, giving a description of rotational behavior of the system in long relaxation time scales, emphasizing always the interplay between both evolutionary processes. Finally, implications in observational studies are suggested. Chapter $\mathbf{5}$ gives the conclusions and further plans. 



\section{Chapter 2}

\section{Theoretical dynamics}

Globular star clusters have become very interesting targets of theoretical studies since the acceptance of their complexity and their important role in the origin and evolution of the universe. They are now far away from the initial concept, that describes them as spherical non-evolutionary objects, as they appeared to be placed in space since their discovery. The interplay of GCs together with their parent galaxies plays an important role in the destiny of galaxies and clusters. And as they are very old objects, they hide still unanswered questions about the origin of galaxies and the universe itself.

Nowadays many new interesting phenomena need to be taken into account in order to construct a consistent model, which could fit in the best way, observations of globular clusters systems in many galaxies. In some sense, observations have gone ahead of theory in the last years, due to the technical improvement of ground-based and satellite telescopes. The task of theoretical research is to overcome this challenge. In this chapter, an overview of theoretical methods, able to study self-gravitating stellar systems, is made. Advantages and disadvantages of each of them are formulated and the selection of the method used in the present work is justified. 


\subsection{Numerical Methods}

Modeling globular cluster evolution has become an important task in describing stellar interactions and evolution in direct relation with cluster and galactic structure. As mentioned in the previous chapter, observations with the Hubble Space Telescope and other modern equipment have revealed fascinating new facts about these galactic building blocks, helping in studying the stellar structure and age of the clusters in relation with the evolution of their parent galaxies as well as giving a lower boundary for the age of the universe (a review can be found in Deiters et al., 2001).

Nevertheless, and despite recent progress in algorithmic and mathematical methods and in hardware development, dynamical modelling of stellar clusters significantly lags behind the wealth of observational data *

Direct $N$-body modelling, where individual orbits of a large number of stars are integrated step-by-step, and the force on each star by all the other stars is calculated directly to high-order accuracy, has made significant progress in the past years: Thus, Fall \& Frenk (1985) performed 500-body simulations; Akiyama \& Sugimoto (1989) performed 1000-body computations; Aarseth \& Heggie (1993) integrated 6000 stars and 180 binaries; Spurzem \& Aarseth (1996) pioneered a 10,000-run computation, and Baumgardt \& Makino (2003) were able to include 130,000 stars. This progress was made possible by significant algorithmic and mathematical discoveries, such as chain-regularisation, ${ }^{\dagger}$ and also through the development of the GRAPE special-purpose hardware in Japan by Jun Makino and collaborators (see Aarseth 1999, for a review). Current models incorporate fully realistic stellar and binary-star evolution as well as realistic 3D tidal fields (Kroupa, Aarseth \& Hurley 2001; Baumgardt \& Makino 2003). They constitute good experimental laboratories for the validity of other methods. Nevertheless and despite this progress, the number of stars that can be used is of the order of a few $10^{5}$ at the very extreme (using the most-powerful special-purpose supercomputers in Japan over months of computational time), and this still does not quite reach realistic stellar numbers in massive GCs.

Dynamical evolution of star clusters has been examined by gaseous sphere models, which use a phenomenological heat flux equation in order to close the moment equations of the Boltzmann equation with a Fokker Planck collision term. Anisotropy is taken into account for the second order moments (radial and tangential velocity dispersions). The physical nature of gravothermal collapse (Lynden Bell \& Eggleton, 1980) and the existence of gravothermal oscillations (Bettwieser \& Sugimoto 1984) were detected using gaseous models. An overview can be found in Spurzem (1996).

The Fokker-Planck method (FP) is based on the Boltzmann equation and using a collisional term, simplifying the physics by making use of the long relaxation processes of

\footnotetext{
* Note that as globular clusters have been treated for long time as spherical systems, spherically symmetric models have been well developed. That is not the case for the non-spherical models, axisymmetric or triaxial, which constitute the target of the present study.

${ }^{\dagger}$ Regularisation method for integrating the equations of motion of N-body systems. A chain of interparticle vectors is selected in such a way that the critical interactions requiring regularisation are included in the chain. The equations of motion for the chain vectors are subsequently regularized using the variables of two-body regularisation method (Kustaanheimo-Stiefel, 1965) and a time transformation.
} 
the problem, under the assumption of slow changes in the potential in a quasi-steady state evolution. This complex multi-dimensional integro-differential equation can itself be solved numerically by employing an orbit-average approximation. An overview can be found in Spurzem (1999). The FP method allows the study of a set of large number of models, because the solution of the underlying equations is usually computationally cheap compared to a full-scale $N$-body simulation. Also, physical understanding of the results of direct $N$ body modelling are improved by a comparison with such methods (Takahashi \& Portegies Zwart 1998; 2000; Preto, Merritt \& Spurzem 2004), and thereby they also help in scaling small direct computations to large $N$.

In the spherical case there is a large coverage of modelling tools. Self-gravitating stellar systems have been extensively theoretically approximated through isotropic systems (Cohn 1980; Heggie 1984) or 2D anisotropic models (Takahashi 1995; 1996; 1997) and also by using different mass bins or even a continuous mass spectrum (Spurzem \& Takahashi 1995; Giersz \& Heggie 1997; Heggie et al. 1998).

Monte-Carlo simulations calculate diffusion of velocities at selected number of testparticles, adding the changes in velocity (random generated according to a probability distribution) and following the distribution of the test-particles in time (Marchant \& Shapiro 1980; Spurzem \& Giersz 1996; Giersz 1998). This method can be applied for dynamical systems and orbit-averaged applications (Giersz 1996). Thus, the Monte Carlo scheme can be regarded as a statistical method which lies in the middle between direct N-body and Fokker-Planck models and combines most advantages of both methods.

An illustration that numerical methods can complement each other leading to a more accurate description of the targets of study can be seen in Fig (2.1), where results of a comparative study on the dynamical evolution of a model star cluster is shown. In this experiment, the evolution of a cluster was specified by given initial and boundary conditions using the widest possible variety of methods. Simulations of N-Body, Fokker Planck and gaseous models were used and the results of diverse structural parameters were put together as in the figure. Recent developments have made possible to achieve more realistic evolutionary models of stellar clusters regarding particle number, initial conditions and evolutionary ingredients like stellar evolution and the existence of primordial binaries. In the next sections a description of rotation and $\mathrm{BH}$ star accretion is made as these are basic elements used for the present study.

\section{$2.2 \quad$ Rotating Models}

Early theoretical studies have shown that angular momentum transport and its distribution play an important role in the destiny of the long term evolution of self-gravitating stellar systems.

Inagaki \& Hachisu (1978) included rotation in the thermodynamic stability of uniformly rotating gaseous cylinders in self-gravitating systems and showed that the system is secular unstable when the temperature is sufficiently low. Instability is mainly caused by the outward transport of angular momentum and is expected to occur in the time scale of the 

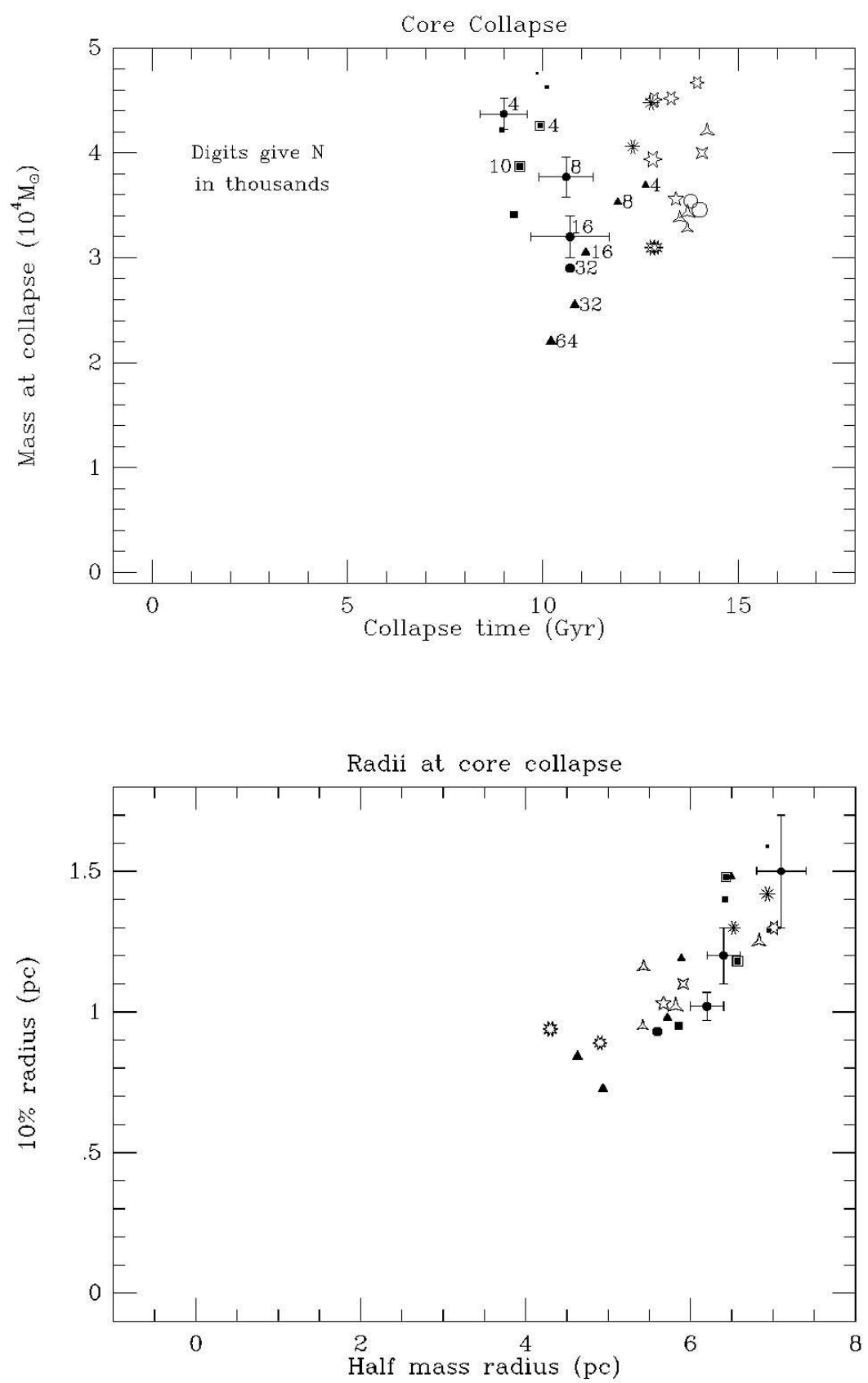

Figure 2.1: Comparative study of the dynamical evolution of a model star cluster at collapse time. The upper panel shows the time and mass at core collapse and the lower panel, the radii at core collapse. Filled symbols represent N-Body results, empty symbols are results of Fokker-Planck methods, and the stars are for gaseous models. From Heggie (2003). 
angular momentum transport. Hachisu (1982) examined infinitesimally thin disks, consisting of gas particles, by means of the linear response theory. He showed that self-gravitating systems are thermally and rotationally unstable, originated by the effects of negative specific heat or negative moment of inertia and by the coupling between them. The rotational instability was called a gravogyro catastrophe, in analogy to the the gravothermal case.

The gravogyro instability sets on with the removing of angular momentum from the core. Then, its angular speed decreases. By the decrease of the centrifugal force, this region contracts towards the axis of rotation by the gravity. As a consequence the moment of inertia decreases, and if this decreasing is large enough, the angular speed becomes greater than its initial value before the removal of angular momentum. Inagaki \& Hachisu formulate the instability in following relation between angular speed $\Omega$ and specific angular momentum $j$

$$
\delta \Omega \approx-\frac{\delta j}{r^{2}}
$$

i.e. the angular speed becomes larger when the core loses its angular momentum and vice versa.

Goodman (1983) shows first, in his unpublished PhD thesis, Fokker Planck models of initially axisymmetric rotating clusters and finds that dynamical relaxation can be the origin of the age-ellipticity relation, like the theoretically predicted by Shapiro \& Marchant (1976) and by Frenk \& Fall (1982, using cluster data from the LMC), in the sense that young clusters are flatter (in average) than the older ones. Goodman applied the orbit averaged Fokker Planck equation (in $E-J_{\mathrm{z}}$ space) to follow the evolution of axisymmetric systems, driven by the effects of weak star-star gravitational encounters from one dynamical equilibrium to another, and could explain the difference in shape between galactic GCs and the youngest LMC clusters. He found that the central angular velocity increases as a weak power of the central density during core collapse and explained it in terms of a linear perturbation of the self-similar solution found by Linden-Bell \& Eggleton (1980) for core collapse in an infinite, non-rotating gas sphere.

Longaretti \& Lagoute (1997) describe an evolutionary model of flattened rotating globular clusters as sequences of quasi-equilibrium King distributions, prior to core collapse. They showed that internal relaxation produces a systematic decrease of cluster concentration and flattening and that the lower limit in initial concentration needed for globular clusters to survive dissolution must be larger than $\Delta W_{0} \sim 1$ (with the reduced potential energy $W$, after King, 1966) for rotating clusters than for their non-rotating counterparts.

The two-dimensional FP models of axisymmetric, rotating clusters obtained by Einsel \& Spurzem (1999) suggest that the theorized gravo-gyro phenomenon (Hachisu 1979; 1982) is a genuine feature of star cluster evolution: Rotation is quickly lost from a cluster through energy-equipartition driven stellar evaporation, so to explain a current cluster rotation, the initial rotation of the system should have been large enough at the beginning. Nevertheless, a seed angular momentum in a proto-cloud would remain in the GC without dissipation, since the time scale of cluster formation is much shorter compared to global relaxation times. However, the expulsion of residual gas (Goodwin 1997; Kroupa, Aarseth \& Hurley 
2001) would rapidly reduce the angular momentum of the surviving expanded cluster core.

Kim et al. (2002) presented first post core collapse models of initially rotating clusters, using the solution of the orbit averaged Fokker Planck equation including the steady threebody binary heating source (e.g., Hut 1985). They confirmed that rotating clusters, tidally limited or not, evolve faster than non-rotating ones.

Kim, Lee \& Spurzem (2004) used 2D FP multi-mass rotating models to show that the presence of initial rotation accelerates the core collapse when compared with an equal-mass system. The acceleration rate of core-collapse due to rotation is larger for models with an initially steeper slope of the mass function, for a fixed dimensionless initial rotation. The enhanced mass loss rate, resulting from the enhanced two-body relaxation process, causes a faster dissolution of the cluster. Due to an interplay of the central concentration of the massive stars and the transfer of angular momentum from more-massive stars to less-massive stars, the radii where the rotational velocity $\left(V_{\text {rot }}\right)$ reaches its maximum value moves inwards.

N-Body simulations have been also applied to the rotating case. Fall \& Frenk (1985) used $\mathrm{N}=400$-systems with initial slow rotating oblate spheroids with a density King profile and observed the decrease of ellipticity in time by a factor of 2 at about $5 \tau_{\text {rh }}$ (the simulations were stopped after $13 \tau_{\mathrm{rh}}$ ), followed by a slower evolution. The initial rotational energy was set to $5 \%$ of the total random kinetic energy. Later, direct $1000-N$-body simulations used by Akiyama \& Sugimoto (1989) found evolutionary phases in rotating globular cluster simulations. These are: an early rapid violent relaxation, gravogyro catastrophe (proceeding in a time scale of two-body relaxation), a leveling off of the gravogyro catastrophe accompanied by gravothermal instabilities and a relatively rapid gravothermal collapse. The cluster developed a the core-halo structure and its shape became secularly rounder.

Thus, rotating models need still further development when one goes to more system complexity and aims to more realistic simulations of clusters. Ingredients as stellar evolution, primordial binaries or realistic galactic tidal boundaries can be added and evaluated in collaborative efforts using the different methods presented in Sect (2.1) or in the form of hybrid codes. One next step, in model development was made using models, which include central (fixed or not) BHs, as presented in the next section.

\subsection{Black Hole Models}

$\mathrm{BHs}$ are particularly interesting for GCs. Their origin is still mysterious and many aspects of their interplay with the surrounding stellar cluster remain to be elucidated.

It was proposed that intermediate-mass black holes (IMBHs) can form in young star clusters (Portegies Zwart et al. 1999). The cluster core is likely to experience core collapse driven by the accumulation of the most-massive stars in the cluster center through dynamical friction (Spitzer 1987), before stellar mass loss drives the expansion of the core and cluster. Thus it seems to be possible to build-up a massive star through runaway collisions, which may ultimately collapse to a IMBH (Portegies Zwart et al. 2002; Gürkan, Freitag \& Rasio 2004). This BH may subsequently capture a stellar companion by tidal effects 
and turn into a bright X-ray source (Hopman et al. 2004). $N$-body simulations of several star clusters in M82 (Portegies Zwart et al. 2004) found that runaway merging of massive stars could have led to the formation of an IMBH with a few hundred to a few thousand solar masses in MGG-11. Apart from run-away collisions of main sequence stars, IMBHs may also be build-up through the merger of stellar-mass BHs (Mouri \& Taniguchi 2002) via gravitational radiation in dense-enough star clusters.

As found by Pebbles (1972), if the relaxation time in the core of the star cluster is less than the age of the system, the star distribution around a collapse object must relax to a unique steady-state shape. Bahcall \& Wolf (1977) found that the equilibrium star density approximates an $r^{-7 / 4}$ power law throughout most of the $1 / r$ gravitational well of the $\mathrm{BH}$. Frank \& Rees (1976) describe quasi steady states, involving a steady influx of low angular momentum 'loss-cone' orbits of stars disrupted (or swallowed) by the BH inside a critical radius $r_{\text {crit }} \ddagger$. The $-7 / 4$ cusp in the density profile was found for $r_{\text {crit }}<r<r_{\mathrm{h}}$, where $r_{\mathrm{h}} \simeq G M_{\mathrm{h}} / v_{\mathrm{c}}^{2}$ is the influence radius of the $\mathrm{BH}$, and flatter inside $r_{\text {crit }}$. One-dimensional FP models where the distribution function $f$ depends only on the energy $E$ of the stars and all physical properties depend only on a radius from the cluster center, were widely used by many other authors (Cohn 1980, Murphy B. 1991, Drukier et al. 1992, among others).

The interplay between an embedded central star-accreting BH and energy transport by two-body relaxation as well as loss-cone accretion of stars on orbits of low angular momentum was treated in self-gravitating systems by many authors. Lightman \& Shapiro (1977) used an analytic analysis of the 2D Fokker Planck equation in energy $E$ and angular momentum $J$ to construct a steady state distribution of stars and calculate the consumption rate of low angular momentum stars orbiting a massive $\mathrm{BH}$ in a spherical system. The equilibrium state is driven by relaxation processes associated with gravitational stellar encounters. Stars enter the loss cone in J-space defined by $J<J_{\min } \sim\left(G M r_{\mathrm{t}}\right)^{1 / 2}$ and are accreted in a dynamical time scale (here $r_{\mathrm{t}} \sim(M / \rho)^{1 / 3}$, where $\mathrm{M}$ is the $\mathrm{BH}$ mass and $\rho$ the stellar density).

Marchant \& Shapiro (1980) used Monte Carlo models to simulate the development in relaxation time scales of isolated star clusters with embedded BHs. They showed that the presence of a relatively small $\left(\gtrsim 50 M_{\odot}\right) \mathrm{BH}$ can halt core collapse and that the growth of the $\mathrm{BH}$ (depending of the accretion efficiency) can lead to significant reexpansion of the core. However, on time scales of relaxation the black hole can not grow beyond $M_{\mathrm{BH}} \sim 4000 M_{\odot}$. Duncan \& Shapiro (1982) performed Monte Carlo simulations of the dynamical evolution of star clusters regarding the effects of a central BH and/or the tidal effects of the Galaxy. They found that cluster evolution with central 'seed' BHs $\left(\approx 100 M_{\odot}\right)$ is qualitatively the same whether the hole is introduced instantaneously during the late phases of core collapse or in the early stages of the evolution. In all cases, the core collapse is halted by the heat flux due to stellar consumption by the hole, and the cluster reexpands, achieving a quasistationary state, while the hole mass increases to several thousands solar masses.

\footnotetext{
${ }^{\ddagger}$ As defined by Frank \& Rees (1976), $r_{\text {crit }}$ is the radius, where the diffusion angle of velocity in a dynamical time scale is comparable to the loss cone angle.
} 
Furthermore, in 2D Fokker Planck models (without BH), two different components of velocity dispersion (velocity anisotropy), which allow more accurate treatment of evaporation of stars residing in the outers regions of the clusters, were included by Takahashi (1995, 1996, 1997). He found that the halo becomes dominated by radial orbits, as the result of strong relaxation in the core, in single-mass as well as in multi-mass cluster models.

Many physical ingredients, such as primordial binaries, binary formation by three-body processes, stellar evolution and mass spectrum, were included in FP models assuming spherical symmetry (Takahashi, Lee \& Inagaki 1997; Takahashi \& Lee 2000). And the contribution of stellar evolution and collisions to the stellar supply to the BH (Murphy et al. 1991; Freitag \& Benz 2002).

Thus, as two-body relaxation generates steady-state density cusps around massive BHs and drives core collapse and gravothermal core oscillations in (globular) clusters, bound stellar systems can achieve an aspherical shape through a non-isotropic velocity field, as well as by rotation (Binney \& Tremaine 1987). Therefore, the combined effect of rotation and relaxation plays an important role in the formation and evolution (specially before the angular momentum is carried away by escaping stars) of dense stellar systems harboring black holes.

Generalizing an earlier work by Young (1980), and motivated by observations of the nearby galactic nuclei M31, M32 and NGC 4594 (Dressler \& Richstone 1988; Jarvis \& Dubath 1988, among others), Lee \& Goodman (1989) studied the evolution of rotating stellar spherical systems with a centered $\mathrm{BH}$ but under the condition of slowly adiabatic $\mathrm{BH}$ growth. This condition is more applicable to galactic nuclei than globular clusters, but as rotation was included it is interesting to mention here. In a rotating system, one expects an increase in $V_{\text {rot }} / \sigma$, since $\sigma$ is an average of $\sigma_{r}$ and $\sigma_{\perp}$, whereas $V_{\text {rot }}$ is entirely due to tangential motion. They found that although the rotation velocity and the velocity dispersion increase when the $\mathrm{BH}$ is added, the rotation velocity increases faster. The ratio $v_{\text {rot }} / \sigma$ was found to rise slowly inwards the $\mathrm{BH}$ influence radius, so that approaches to 1 in the center. Note that dynamical relaxation was not part of this simulations, as is expected in globular clusters.

After angular momentum loss in globular star clusters, remnant angular momentum can be inferred from the flattening and in direct measurements of rotational velocity. The observed steep rise of $V_{\text {rot }} / \sigma$ near the cluster center (see Section 1.3) could, nevertheless, not be explained by a single rotating mass model (Kim et al. 2002) or with the current multi-mass models (Kim, Lee \& Spurzem 2004), although a moderate increase on this ratio was found here (see also Einsel, 1998). Therefore one of the aims of this work is to study the effects of rotation in the dynamical evolution of rotating stellar clusters, embedding intermediate-mass BHs. A modification in the rotational profile is expected due to the presence of the $\mathrm{BH}$ already at early evolutionary times, before angular momentum is carried away by escapers. 


\subsection{Tidal fields}

Clusters evolving in a galaxy are truncated by the galactic tide and evaporate across the tidal boundary as stars keep being elevated to high-energy orbits through rare energetic two- or more-body encounters and through frequent but weak distant encounters (energy equipartition). Because the physical size of a cluster diminishes with its mass, excessive mass loss can lead to the disruption of a cluster as it orbits around the galaxy (Meylan \& Heggie 1997).

A standard boundary condition applied to spherical clusters in FP models is a cutoff of the distribution function in binding energy, as for the King models. Takahashi and Portegies Zwart (1998) achieved good agreement between FP and $N$-body computations by using an anisotropic FP model with an improved escape criterion (apocentre criterion). They showed that anisotropy plays an important role in the outer parts of the system. For GCs on eccentric orbits, dynamical shocks due to the disk and bulge passages enhance their disruption (Gnedin \& Ostriker 1997). Gnedin, Hernquist \& Ostriker (1999) and Gnedin, Lee \& Ostriker (1999) provide an approximate scheme with tailored quasi-diffusion coefficients to include disk and bulge shocking, but only use simple 1D FP models. Furthermore, Kim et al. (2002) showed that tidally limited rotating 2D-FP models reach core-collapse earlier than isolated ones.

\subsection{Resume}

Dynamical modelling of stellar clusters lags behind the wealth of observational data, despite recent progress in algorithmic and mathematical methods and in hardware development. Moreover, the existence of a variety of numerical methods (N-Body, gaseous models, FokkerPlanck, Monte-Carlo), permits to complement each other, by applying them to the same problem, and leading to a more accurate description of the targets of study.

Modelling of stellar clusters, in the spherical approximation has been widely theoretically developed and verified with observational analyses. Non-spherical models need still further development, specially when one goes to more system complexity and realism. Ingredients as stellar evolution, primordial binaries or realistic galactic tidal boundaries have been added and evaluated in collaborative efforts using the different methods presented in Sect (2.1) or in the form of hybrid codes. A next step in model development is carried on in the present work, by the implementation of a central fixed $\mathrm{BH}$ together with system rotation. Intermediate-mass black holes (IMBHs) probably exist in star clusters, and may have formed in young clusters (like in the GCS of the LMC). Theoretically, the interplay between an embedded central star-accreting $\mathrm{BH}$ and energy transport by two-body relaxation as well as loss-cone accretion of stars on orbits of low angular momentum was treated in self-gravitating spherical systems by many authors. The non-spherical approximation including accreting BHs could modify the rotational profile already at early evolutionary times, before angular momentum is carried away by high energetic stars.

Another element in globular cluster modelling is their evolution in a galaxy tidal field, which causes evaporation of stars across the tidal boundary, while stars keep being elevated to high-energy orbits through two- or more-body encounters and through frequent but weak 
distant encounters. The common evolution cluster-galaxy should be well understood, due to the key role of GC evolution in the explanation of galaxy evolution and formation. 


\section{Chapter 3}

\section{Model}

In principle, all simulation methods presented in Section (2.1) are applicable to globular clusters. Some of the advantages of each of them were mentioned in the previous Section. The Fokker-Planck method used for this study makes possible a detailed analysis of angular momentum transport using the orbit-averaging, described in the following section. Specially for the problem of black hole star accretion the advantage is due to the fact, that this phenomena is fully driven by angular momentum diffusion and the loss cone is defined using angular momentum limits. Although the several improvements in N-Body simulations in the last years, as described in Sect. (2.1), the high particle number of globular star clusters $\left(\gtrsim 10^{6}\right)$ can still hardly be achieved, and the statistical Fokker Planck solution gives a good alternative to N-Body direct integration. Moreover, statistical models can be straightforward used as complement and test of small N-Body simulations and make possible extrapolation of the results to higher particle numbers. On the other side, FokkerPlanck methods can use the advantages of the exact N-Body solution to confirm statistical approximations and deal with more complexities.

The method developed by J. Goodman (1983) applied to axisymmetric systems and the improvements by C. Einsel (1999) and E. Kim $(2002,2004)$ are the basis of the present study and further development of the code, as a continuation of a project, aimed to underline the relevance of the role of rotation in the dynamical evolution of stellar clusters containing central star accreting black holes. The method is described in the following, placing emphasis in the treatment of the $\mathrm{BH}$ problem in the frame of a brief description of the general background of the Fokker-Planck method. 


\subsection{Fokker Planck equation}

The Fokker-Planck method has been extensively used in modelling self gravitating systems in the spherical case. The one dimensional (Cohn 1980, Lee et al. 1991, Murphy B. 1993, Drukier et al. 1992, among others) and $E, J$ two-dimensional case (Lightman \& Shapiro 1977; Takahashi, Lee \& Inagaki 1997; Takahashi \& Lee 2000) have been well developed and many physical ingredients, such as primordial binaries, binary formation by three-body processes, stellar evolution and mass spectrum were included.

The pioneering work of Goodman (1983), in his unpublished thesis, and the further development of the Fokker-Planck method made by Einsel \& Spurzem (1999) and Kim et al. $(2002,2004)$, have brought the treatment of the axisymmetric rotating case to a newly state of interest, which follows the evolution of self-gravitating rotating systems driven by relaxation effects and its consequences for the stellar redistribution and shape of the system.

In this Section, a further development of the method is described, with the aim to predict the destiny of rotating globular cluster systems, which contain a central embedded star accreting black hole. The influence of the central potential in the redistribution of orbits specially in the very center of the system is of interest, but the implications for non-bounded orbits need also to be elucidated, as they can be lost through the tidal boundary generated by the potential of a parent galaxy, around which the cluster orbits, accelerating the dissolution of the cluster.

Thus, the method described in this section, is seen as the next step in the aim to simulate the evolution of a realistic cluster, as observational evidences of possible BHs in center of globular clusters exist. Nevertheless, simplifications are made in order to concentrate in the interplay between rotation, relaxation and $\mathrm{BH}$ accretion in the time evolution of the stellar system.

\subsubsection{Equations and assumptions}

Evolution of the distribution function $f_{a}(\vec{r}, \vec{v})$ of stars in phase space $(\vec{r}, \vec{v})$ under the influence of the potential $\phi(\vec{r})$ is described by the Boltzmann-equation

$$
\frac{\partial f_{a}}{\partial t}+\vec{v} \cdot \vec{\nabla}_{\mathrm{r}}+\frac{\vec{F}}{m_{\mathrm{a}}} \cdot \vec{\nabla}_{\mathrm{v}} f=\left(\frac{\partial f_{a}}{\partial t}\right)_{\mathrm{coll}}
$$

with space and velocity coordinates, $\vec{r}$ and $\vec{v}$ respectively. The index $a$ denotes the stars of mass $m_{\mathrm{a}}$ at which the force $\vec{F}=-m_{\mathrm{a}} \vec{\nabla}_{\mathrm{r}} \phi$ is applied. The potential at infinity is set to zero, and is negative at inner radii $(\phi \leq 0)$. The term on the right side of equation (3.1) takes into account the changes in $f$ due to collisions (not real collisions but stellar scatterings which cause deviations in the orbits). It allows the compressibility of the stellar flux in phase space, and the change in phase space density the neighborhood of stars. It is called the 'Fokker Planck' term, as the left side of equation 3.1 is called the 'Vlasov' term.

The collision term is given through the -local- Fokker Planck approximation

$$
\left(\frac{\partial f_{\mathrm{a}}}{\partial t}\right)_{\text {coll }}=-\frac{\partial}{\partial v^{\mu}}\left(f_{\mathrm{a}}\left\langle\Delta v^{\mu}\right\rangle_{\mathrm{a}}\right)+\frac{1}{2} \frac{\partial^{2}}{\partial v^{\mu} \partial v^{\nu}}\left(f_{\mathrm{a}}\left\langle\Delta v^{\mu} \Delta v^{\nu}\right\rangle_{\mathrm{a}}\right)
$$


where $\mu=1,2,3$ and $\nu=1,2,3$ (tensor notation). $v^{\mu}$ gives the velocity in Cartesian coordinates and $\left\langle\Delta v^{\mu}\right\rangle_{\mathrm{a}}$ the diffusion coefficient (average increasing of velocity through collisions per unit time). The first order diffusion coefficients $\left\langle\Delta v^{\mu}\right\rangle_{\text {a }}$ describe the dynamical friction (negative due to the contrary direction of slow down of the velocity of a star in a background stellar field). The second order diffusion coefficients $\left\langle\Delta v^{\mu} \Delta v^{\nu}\right\rangle_{\text {a }}$ give the real velocity diffusion.

In order to obtain the solution of the Fokker Planck equation following assumptions are taken into account:

- cluster evolution time scales are in following relation:

$$
t_{\text {dyn }} \ll t_{\text {rh }} \ll t_{\text {diss }}
$$

where times are the dynamical, half mass relaxation and dissolution times scales respectively, defined in Sect. 1.1. Note that the relaxation time at the BH influence radius is taken as time unit for the present calculations (see Eq. 3.10)

- the solution is given for small angle scatterings $(\Delta v / v \ll 1)$, i.e., for changes of $\vec{v}$ to $\vec{v}+\Delta \vec{v}$.

- higher order diffusion coefficients are neglected because they are much smaller than the first or second order ones (Hénon 1960, 1973).

- there is no correlation between collisions (like in three body collisions), which could be important for the energy generation in the core that can reverse the collapse. This effect is here not considered, but in fact the $\mathrm{BH}$ potential represents an energy source included in our models.

Regarding the stellar distribution around the central BH and its central accretion, following general assumptions are taken:

- The distribution of stars is represented by a single-particle system that is initially axisymmetric in space and anisotropic in velocity space. No stellar spectrum is included in this model. Although, the implications of this constraint could be important to be considered, this will allow to make a first test of the model without large complexity. Expected deviations in the evolution caused by different mass bins are discussed in the next chapter.

- the initial $\mathrm{BH}$ mass, $M_{\mathrm{BH}}$, is much smaller than the cluster mass $M_{\mathrm{cl}}$

- no binaries and stellar evolution is considered. The lack of stellar evolution implies that no star gas accretion is possible, as would be for globular star clusters in their evolution on dynamical time scales. The presence of binaries in the cluster is not considered in order to simplify the conditions and follow carefully the evolution of $\mathrm{BH}$ star accretion under the influence of rotation and relaxation. Binary heating can reverse collapse as the presence of $\mathrm{BH}$.

- a star is disrupted if its z-component of angular momentum is less than a minimum angular momentum $J_{\mathrm{z}}^{\min }$, which defines the loss-cone boundary (see Sect. 3.2.2) 


\subsubsection{Integrals of motion}

An integral of the motion is any function of $\vec{r}$ and $\vec{v}$ that remains constant along any stellar orbit. Isolating integrals confine the orbit to some restricted region of phase space, whereas nonisolating integrals do not (a star with a given value of a non-isolating integral can come arbitrarily close to any point in phase space). Thus, $f(\vec{r}, \vec{v})$ cannot be a function of nonisolating integrals (since a point $(\vec{r}, \vec{v})$ corresponds arbitrarily closely to any value of the nonisolating integral, but is clearly associated with a single well-defined value of $f$ ). In fact, it can be shown that $f(\vec{r}, \vec{v})$ is a function only of isolating integrals of the motion (Jeans' theorem). The solution of the Fokker-Planck equation uses the simplification given by the conservation of the integrals. In spherically symmetric systems and in dynamical equilibrium, with isotropy in the velocity distribution depends only on the energy and time $(f(E, t))$. When anisotropy in the velocity distribution is allowed, four integrals of motion exist: the energy and the three components of angular momentum (and consequently, the absolute value of $J$ is also an integral).

The classical isolating integrals of a general axisymmetric potential $\phi$, in cylindrical coordinates $(\varpi, z)$, are the energy per unit mass:

$$
E=\frac{1}{2} v^{2}+\phi_{c l}(\varpi, z)+\phi_{b h}(\varpi, z)
$$

where $\phi_{c l}(\varpi, z)$ is the potential of the stellar system and $\phi_{b h}(\varpi, z) \equiv G M_{b h} / r$ is the BHpotential $\left(r^{2}=\varpi^{2}+z^{2}\right)$; and the component of angular momentum along the z-axis per unit mass, given by

$$
J_{z}=\varpi \vec{v} \hat{e}_{\varphi}
$$

$\vec{v}_{\varphi}=v \hat{e}_{\varphi}$ is the velocity component in azimuthal direction.

The analytical expression for $\phi_{b h}$ permits its accurate calculation in the code and always possible separation of $\phi_{c l}$, as $\phi(\varpi, z)=\phi_{b h}(\varpi, z)+\phi_{c l}(\varpi, z) . E$ and $\phi$ are negative for bound particles.

Note that the velocity components $\left(v_{\varpi}, v_{\mathrm{z}}, v_{\phi}\right)$ constitute a Cartesian coordinate system in velocity space, in the axisymmetric, not Cartesian coordinate system $(\varpi, z, \varphi)$.

Conservation of energy and z-component of angular momentum is used in the solution of the Fokker Planck equation, which becomes a non-linear second order integro-differential equation, because the diffusion coefficients of Eq. (3.2) are expressed in terms of integrals over the local field star velocity distribution function $f$. This integrals are given by the Rosenbluth potentials (Rosenbluth et al., 1987). A derivation of the diffusion coefficients in terms of $E$ and $J_{\mathrm{z}}$ can be found in Einsel \& Spurzem (1999).

Although $f$ can be approximately representable as a function of $E, J_{z}$ and $t$ (except for very special forms of the potential), numerical evidence demonstrates, that axisymmetric 
potentials can support orbits which have three integrals of motion: $E, J_{\mathrm{z}}$ and a third integral commonly designated $I_{3}$. That is, the typical orbit does not spread uniformly over the hypersurface in phase space defined by its $E$ and $J_{z}$ but is confined to lower-dimensional subset ('non-ergodic' orbits on their $E J_{z}$ surfaces). Gerhard \& Binney (1985) find a third integral by computing individual orbits and plotting their intersections in areas in phase space. Given $E$ and $J_{z}$, they found closed curves in phase space, and thus regular orbits, only explicable by the existence of a third integral. This is the method of surface of section, which in the case of non-existence of $I_{3}$, would lead to chaotic intersected areas in phase space. A solution of the orbit-averaged FP equation in energy-momentum space may represent an artificial case of a true point-mass system, since in the axisymmetric potential a third integral of motion could restrict particle motion in phase space (Goodman, 1983).

The use of $J^{2}$ as an approximation of the third integral by Lupton \& Gunn (1987) in spherically symmetric systems demonstrate, that for a light flattened system, the distribution function along an orbit, varies roughly $5 \%$, if $E, J_{\mathrm{z}}$ and $J^{2}$ are given. On one side, the inner parts of the cluster are dominated by relaxation effects and the third integral can be neglected, due to the efficiency of diffusion in these regions, which ensures ergodic distribution of orbits in phase space in an area given by $\left(E, J_{z}\right)$. On the other side, the outer region of the cluster can be strong influenced by the third integral, as radially biased anisotropy dominates this region.

In the present study, non-ergodicity on the hypersurface (given by $E$ and $J_{z}$ ) is neglected due to any third integral $I_{3}$. Furthermore, the potential close to the BH is spherically symmetric $(\sim 1 / r)$, and $I_{3}$ could be fairly approximated by $J^{2}$, but as tangentially biased anisotropy is expected in this regions (less radial orbits), the angular momentum is here good represented by $J_{\mathrm{z}} \sim J_{\mathrm{z}}^{\max }$. Nevertheless, radial and possible existing meridional circular orbits would not be distinguished by the model, due to the absence or the low values of $J_{\mathrm{z}}$ in both cases, and will be treated as radial orbits (for example when they are redistributed in energy space due to changes in the potential, Sect. 3.3.2). Moreover, accretion of high energy, low angular momentum orbits will leave a small fraction of them in the cluster center. See Section 3.2.1 for a further discussion about the implications of neglecting third integral.

In terms of integrals of motion, the Boltzmann equation is expressed in the axisymmetric system as:

$$
\frac{\partial f}{\partial t}+\frac{\partial \phi}{\partial t} \frac{\partial f}{\partial E}=\left(\frac{\partial f}{\partial t}\right)_{\text {coll }}
$$

The dependence on $J_{\mathrm{z}}$ is given implicitly by $\phi$; and the collisional term can be expressed in terms of $E$ and $J_{\mathrm{z}}$ as

$$
\begin{gathered}
\left(\frac{\partial f}{\partial t}\right)_{\text {coll }}=\frac{1}{V}\left[-\frac{\partial}{\partial E}(\langle\Delta E\rangle V f)-\frac{\partial}{\partial J_{\mathrm{z}}}\left(\left\langle\Delta J_{\mathrm{z}}\right\rangle V f\right)+\right. \\
\left.+\frac{1}{2} \frac{\partial^{2}}{\partial E^{2}}\left(\left\langle(\Delta E)^{2}\right\rangle V f\right)+\frac{1}{2} \frac{\partial^{2}}{\partial J_{\mathrm{z}}}\left(\left\langle\Delta E \Delta J_{\mathrm{z}}\right\rangle V f\right)+\frac{1}{2} \frac{\partial^{2}}{\partial J_{\mathrm{z}}^{2}}\left(\left\langle\left(\Delta J_{\mathrm{z}}\right)^{2}\right\rangle V f\right)\right]
\end{gathered}
$$


the volume element in velocity space is given by $V=\frac{2 \pi}{\pi}$, which needs to be integrated over a third velocity component (here the azimuth $\psi$ of $\vec{v}$ with respect to $\hat{e}_{\varphi}$ measured in the $\varpi z$ plane counterclockwise from the direction $\hat{e}_{\varpi}$ ).

\subsubsection{Units and $E, J_{z}$-grid}

The system of units is given by

$$
G \equiv M_{\mathrm{i}} \equiv r_{\mathrm{c}_{\mathrm{i}}} \equiv 1
$$

where $G$ is the gravitational constant, $M_{\mathrm{i}}$ the initial mass of the cluster and $r_{\mathrm{c}_{\mathrm{i}}}$ the initial core radius (Eq. 1.7). It permits at every time, after fixing them to real physical units, a rescaling of the system to allow comparison with observations.

As the mass of the $\mathrm{BH}\left(M_{\mathrm{BH}}\right)$ is also given in units of $M_{\mathrm{i}}$, a re-rescaling is, in this case, given by just fixing the initial cluster mass, and same for all masses, as the mass at the core radius or at the Lagrangian radii.

As mentioned in Section 1.1, cluster evolution time scales need to maintain in following relation:

$$
t_{\text {dyn }} \ll t_{\text {rh }} \ll t_{\text {diss }}
$$

where the times are the dynamical, half mass relaxation and dissolution times scales respectively, defined in this section.

The unit of time is proportional to the local relaxation time at $r_{\mathrm{a}}$, the influence radius of the $\mathrm{BH}$, defined as the radius, where the mass of the cluster equals $M_{\mathrm{bh}}$. The time step is given by $\Delta t=\xi(t) \tau_{\text {ra }}$, where:

$$
\tau_{\mathrm{ra}}=\frac{0.338 \sigma_{\mathrm{a}}^{3}}{n\left(r_{a}\right)(G m)^{2} \ln \Delta} \quad(\text { Spitzer \& Hart }, 1971)
$$

$\sigma_{\mathrm{a}}$ is the velocity dispersion evaluated at $r_{\mathrm{a}}, G$ is the gravitational constant $(G \equiv 1), \ln \Delta$ is the Coulomb logarithm, where $\Delta=0.4 N_{a}$ is used. ( $N_{a}$ : number of stars inside $r_{a}$ ) and $\mathrm{m}=1 / \mathrm{N}$ represents the mean mass of particles. The initial $\xi(0)$ depends on the initial King parameter $\left(W_{0}\right)$ and is increased from time to time in order to have a fractional increasing of central density of between 4 and $6 \%$ per time step.

The vast dynamical parameter range of relaxed cluster systems was specially treated applying appropriate boundary conditions at the inner potential cusp of the BH and the outer cluster tidal boundary (due to the parent galaxy). A double-logarithmic $(\varpi, z)$ space grid is used, and the Fokker-Planck equation will be written in a dimensionless flux form by introducing the dimensionless energy

$$
X(E) \equiv \ln \left(\frac{E}{2 \phi_{c}-E_{0}-E}\right) ;
$$


$E_{0}$ is the critical energy of the system (see definition below), and the dimensionless zcomponent of angular momentum

$$
Y\left(J_{z}, E\right) \equiv \frac{J_{z}}{J_{z}^{\max }} .
$$

were used, with a higher resolution in the center and at high energy (and low angular momentum) levels, where the relation is nearly linear, as well as in the outer parts of the system (halo), where the proportionality $X-\ln |E|$ improves the spacing of the radii of circular orbits with given energies in the direction of the tidal boundary.

For any specified energy the z-component of the angular momentum is normalized to the maximum angular momentum for that energy, which again is the angular momentum of the corresponding circular orbit as a function of E. For each time step $r_{\text {circ }}(E)$ and $J_{z, 0}(E)$ are determined from the evolving potential in the equatorial plane by a simple Newton-Raphson scheme, using the relations:

$$
\left(E-\phi\left(\varpi_{\text {circ }}, z=0\right)\right)=\frac{1}{2} \varpi_{\text {circ }} \frac{\partial \phi}{\partial \varpi}
$$

in order to get $\varpi_{\text {circ }}$ (which is here equal to $r_{\text {cric }}$, due to $z=0$ ), and computing $J_{z, 0}(E)$, using:

$$
\left(J_{\mathrm{z}, 0}(E)\right)^{2}=r_{\text {circ }}^{3} \frac{\partial \phi}{\partial r}
$$

\subsection{Difussion and loss-cone limit}

The diffusion coefficients are calculated in cylindrical axisymmetric velocity components $\left(v_{\varpi}, v_{z}, v_{\varphi}\right)$ and expressed after transformation in terms of integrals of motion $\left(E\right.$ and $\left.J_{z}\right)$. See the Appendix for their derivation. These coefficients are local, i.e. they describe the diffusion in phase space for a set of $(\varpi, z)$ in the meridional plane. Note that non-circular orbits in the equatorial plane experience different diffusion in the meridional plane, what makes necessary an average over the ensemble of stellar orbits (see below).

\subsubsection{Orbit average and flux conservation}

Given the values of $E$ and $J_{z}$, the orbit average of the Fokker Planck equation in the form of equation 3.6 is obtained by integrating it over an area $P\left(E, J_{z}, t\right)$ of the hypersurface in phase space, given by

$$
P\left(E, J_{\mathrm{Z}}, t\right)=4 \pi^{2} \iint_{A\left(E, J_{\mathrm{Z}}\right)} d \varpi d z
$$

this weighting factor gives also the number of stars in the system taking part in the diffusion as

$$
N\left(E, J_{\mathrm{z}}, t\right)=P\left(E, J_{\mathrm{z}}, t\right) \cdot f\left(E, J_{\mathrm{z}}, t\right)
$$


$A\left(E, J_{\mathrm{z}}\right)$ is given by the intersection of the hypersurface with the $\varpi z$ plane, where the sum of the squares of the velocity components in the meridional plane are non-negative:

$$
A\left(E, J_{\mathrm{z}}\right) \equiv\left\{(\varpi z) \mid \frac{1}{2} \vec{v}_{\varpi}^{2}+\vec{v}_{z}^{2}=E-\phi-\frac{J_{\mathrm{z}}^{2}}{2 \varpi^{2}} \geq 0\right\}
$$

This area is symmetric about the $\varpi$-axis if $\phi$ is symmetric about the equatorial plane, and is connected if the isopotentials of $\phi$ are convex with respect to lines parallel to the $z$-axis (held conditions). The condition (3.17) is rastered numerically in the code by given $E$ and $J_{\mathrm{z}}$.

In a general axisymmetric potential, almost none of the orbits are closed, so that the orbital period is not well defined. There exist two different epicycle periods, one each for oscillations in the $\varpi$ and $z$ directions. The orbit average is taken over a time that is larger than both and is the time required for the orbit to spread uniformly over the area $A\left(E, J_{z}\right)$, only because of encounters, i.e. in a relaxation time scale (if the third integral is well conserved). It makes the orbit average an average over sets of particles sharing the same $E$ and $J_{\mathrm{z}}$ but possibly having different $I_{3}$ 's.

In Fig (3.1) evolution in time of the areas $A\left(E, J_{\mathrm{z}}\right)$ are shown for an initial model $\left(6.0,0.6,5 \cdot 10^{-6}\right)$ using fixed energy and angular momentum grid cells (cell number: 30,40 respectively). Note that the $E$ and $J_{\mathrm{z}}$ themselves change in time, only the grid cell numbers are fixed. A spherically symmetric potential coincident with the one in the equatorial plane was calculated and $J^{2}$ used as third integral approximation in order to get the areas $A\left(E, J_{\mathrm{z}}, J^{2}\right)$. These are contained in the $A\left(E, J_{\mathrm{z}}\right)$ 's, which can be constructed as overlap of the $A\left(E, J_{\mathrm{z}}, J^{2}\right)$ 's using different $J$ 's to be chosen between $J_{\mathrm{z}}$ and $J_{\mathrm{z}}^{\max }$ (Einsel, 1997). In Fig. (3.1) $J=\left(J_{\mathrm{z}}+J_{\mathrm{z}}^{\max }\right) / 2$ was always used. As can be seen in the figure, the $A\left(E, J_{\mathrm{z}}, J^{2}\right)$ (filled with vertical lines) areas show deviations from the $A\left(E, J_{z}\right)$ (blue coloured) in zenithal direction, thus the latter can not be constructed as superpositions of the former areas, using different $J$ 's. This deviations give the error in the approximation of $I_{3}$ through $J^{2}$. At later times, when the $\mathrm{BH}$ potential dominates the central parts of the cluster (higher $|E|$ 's), and the system becomes spherical, the shape of the areas approximate closer each other and the constant grid cells move closer to the center (note the smaller space coordinates in the lower panels), as orbit energies increase towards the potential cusp and angular momentum gets smaller.

The Fokker-Planck equation is solved numerically in flux conservation form, following :

$$
\frac{d f}{d t}=\frac{1}{p}\left(-\frac{\partial F_{X}}{\partial X}-\frac{\partial F_{Y}}{\partial Y}\right)
$$

$p$ is the phase volume per unit $X$ and $Y$, with particle flux components in the $X$ and $Y$ directions:

$$
\begin{gathered}
F_{X}=-D_{X X} \frac{\partial f}{\partial X}-D_{X Y} \frac{\partial f}{\partial Y}-D_{X} f \\
F_{Y}=-D_{Y Y} \frac{\partial f}{\partial Y}-D_{Y X} \frac{\partial f}{\partial X}-D_{Y} f
\end{gathered}
$$


a) $t / t_{\text {rhi }}=1.94$

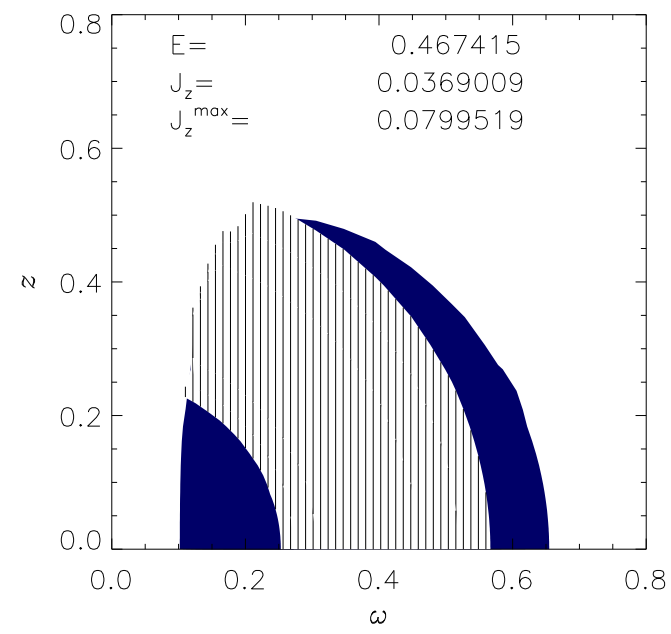

c) $t / t_{r h i}=4.89$

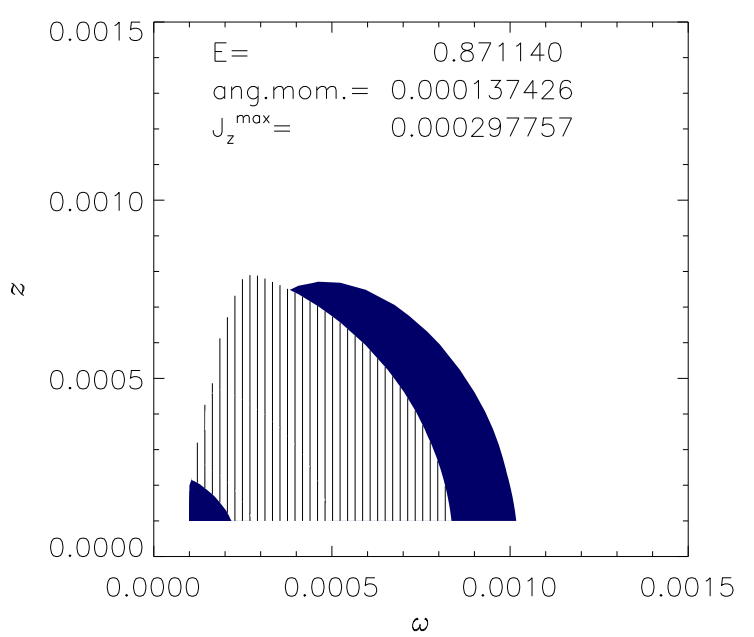

b) $t / t_{\text {rhi }}=3.19$

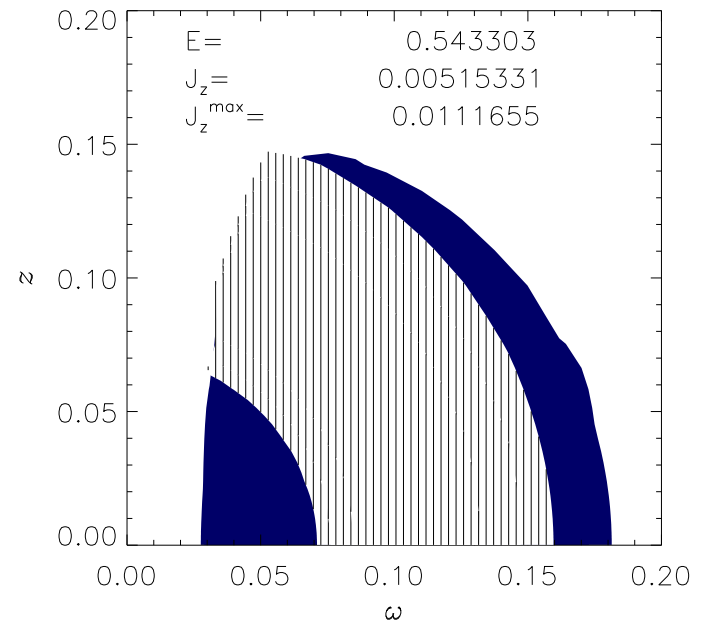

d) $t / t_{r h i}=5.44$

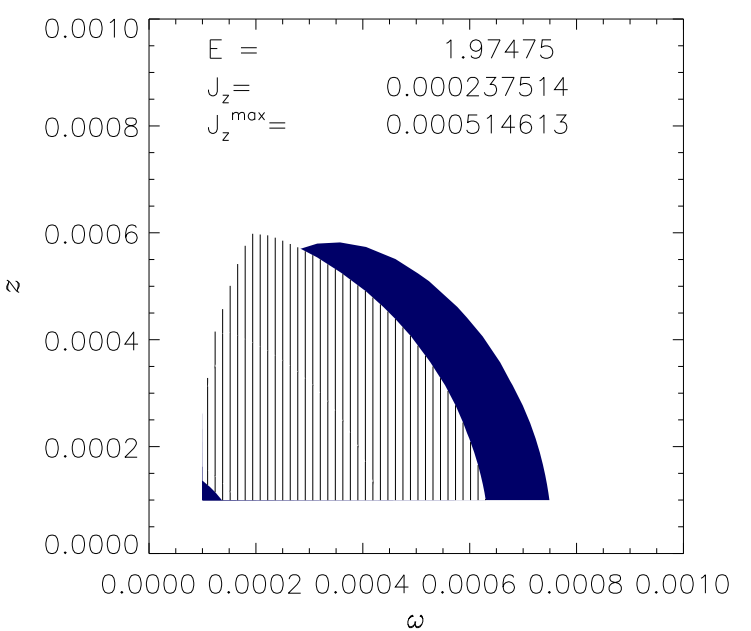

Figure 3.1: Evolution of areas $A\left(E, J_{\mathrm{z}}\right)$ (blue coloured) for an initially rotating flattened model $\left(6.0,0.6,5 \cdot 10^{-6}\right)$ using always the same E-grid cell (number 30$)$ and $J_{z}$-grid cell (number 40$)$. The third integral is approximated by $J=\left(J_{\mathrm{z}}+J_{\mathrm{z}}^{\max }\right) / 2$ and the $A\left(E, J_{\mathrm{z}}, J^{2}\right)$ 's are overploted (vertical line areas) 
The orbit-averaged flux coefficients $D_{i i}$ are derived from the local diffusion coefficients and transformed to dimensionless variables $D_{\mathrm{X}}, D_{\mathrm{Y}}, D_{\mathrm{XX}}, D_{\mathrm{YY}}, D_{\mathrm{XY}}$, as well as the flux coefficients, following

$$
\begin{gathered}
F_{X}=\left|\begin{array}{cc}
\frac{\partial E}{\partial X} & \frac{\partial E}{\partial Y} \\
\frac{\partial J_{z}}{\partial X} & \frac{\partial J_{z}}{\partial Y}
\end{array}\right|\left(\frac{\partial X}{\partial E} F_{E}+\frac{\partial X}{\partial J_{\mathrm{z}}} F_{J_{z}}\right) \\
F_{Y}=\left|\begin{array}{cc}
\frac{\partial E}{\partial X} & \frac{\partial E}{\partial Y} \\
\frac{\partial J_{z}}{\partial X} & \frac{\partial J_{z}}{\partial Y}
\end{array}\right|\left(\frac{\partial Y}{\partial E} F_{E}+\frac{\partial Y}{\partial J_{\mathrm{z}}} F_{J_{z}}\right)
\end{gathered}
$$

The Jacobi matrix is equal to $J_{\mathrm{z}}^{\max }(E) \cdot(d E / d X)$, and the phase space factor transforms as

$$
p^{\prime}(X, Y)=J_{\mathrm{z}}^{\max }(E) \cdot \frac{d E}{d X} \cdot p\left(E, J_{\mathrm{z}}\right)
$$

Derivation of flux terms and coefficients is given in the Appendix.

\subsubsection{Loss cone accretion}

The loss-cone limit is defined by the minimum angular momentum for an orbit of energy E:

$$
J_{\mathrm{z}}^{\min }(E)=r_{d} \sqrt{2\left(E-G M_{\mathrm{bh}} / r_{d}\right)}
$$

where $r_{d}$ is the disruption radius of the $\mathrm{BH}$, calculated as follows (Frank \& Rees, 1976):

$$
r_{\mathrm{d}} \propto r_{*}\left(M_{\mathrm{bh}} / m_{*}\right)^{1 / 3}
$$

$r_{*}$ and $m_{*}$ are the stellar radius and mass, respectively. The disruption radius $\left(r_{\mathrm{d}}\right)$ leads to the definition of the disruption energy $E_{\mathrm{d}} \equiv G M_{\mathrm{bh}} / r_{\mathrm{d}}$. Note that the galaxy tidal energy is denoted by $E_{\text {tid }}$ (Sect. 3.2.3), to distinguish it from the tidal disruption energy of the BH.

A detailed description of the treatment of diffusion due to collisions and the solution of the discretized Fokker-Planck equation is presented in Einsel \& Spurzem (1999). Here the loss-cone implementation is treated.

As the Fokker-Planck equation is solved in order to account for relaxation in the system, the central potential cusp of an embedded massive $\mathrm{BH}$ disturbs the redistribution of stars due to collisional interactions. Thus, following assumptions are taken in order to develop the structure parameters of the cluster:

- accretion is driven by angular momentum diffusion. Energy diffusion is neglected because the changes in $E$ due to collisions are small in comparison to the changes in angular momentum.

- after $\mathrm{BH}$ star accretion $f\left(E, J_{\mathrm{z}}\right)$ needs to be recalculated in order to evolve density, dispersions and rotation 
- the distribution function vanishes for $J_{\mathrm{z}}>J_{\mathrm{z}}^{\max }$ and $J_{\mathrm{z}}<-J_{\mathrm{z}}^{\max }$, but not at $J_{\mathrm{z}}=0$, even for the highest allowed energies.

- the central BH grows slowly leading to always a higher black hole mass (corresponding to the new $\left.f\left(E, J_{z}\right)\right)$ and to a new cluster potential.

- a seed initial BH mass is calculated numerically using a first perturbation of the initial models (see below)

A schematic diagram of the numerical $X Y$-grid (normalized units of energy and zcomponent of angular momentum, Eqs. 3.11 and 3.12), as used for the solution method of the Fokker-Planck equation in discretized form (Eq. 3.38), is shown in Fig. (3.2). Energy limits are the central potential $\left(X\left(\phi_{\mathrm{c}}\right)\right)$ and the tidal energy $\left(X\left(E_{\text {tid }}\right)\right)$, angular momentum limits are the maximum values of z-component of $J$ in both directions $(Y= \pm 1)$. Only the upper half of the grid is shown $(0 \leq Y<+1)$, as the lower half is symmetric with respect to the $Y=0$-axis, for the purpose of this illustration, and corresponds to negative values of $Y(-1<Y<0)$. The loss-cone is represented as blue-shaded area in $(X, Y)$-space. It is limited by $Y_{\min }(E)$ (Eq. 3.24 in normalized units) and the limit of maximum energy $\left(E\left(\phi_{c}\right)\right)$. Thus, the $Y=0$-axis, which appears to be a boundary in this figure, is not a real one. Angular momentum diffusion of stars into and out of neighbor grid cells is illustrated in the right part of the figure (here only in $Y$-direction). The distribution function itself is defined in the center of each cell. In order to solve the system of equations in discretized form (Eq. 3.38), the diffusion terms, in energy and z-component of angular momentum, are computed at the boundary of each grid cell $(X, Y)$.

During the solution method, the hole grid is rastered, in order to account for the contribution of $f(X, Y)$ to loss-cone accretion and the angular momentum fluxes $F_{\mathrm{Y}}$ are computed each time step. Energy diffusion to the loss cone in the horizontal direction is neglected. Moreover, stars are able to escape the cluster through the tidal boundary at $X\left(E_{\text {tid }}\right)$ due to the potential field of the parent galaxy. At this boundary, energy flux takes place through the boundary.

The diffusion flux term $F_{Y}$, per unit energy and unit time across the angular momentum boundary, used to compute diffusion into the loss-cone, is given by

$$
F_{Y}=-D_{Y Y} \frac{\partial f}{\partial Y}
$$

The dimensionless angular momentum flux of stars through the grid cell boundaries, due to diffusion in the inner/outer direction is given by:

$$
\left(\frac{\Delta f}{f}\right)_{\mathrm{acc}}=\frac{\Delta t}{P} \frac{F_{\mathrm{Y}}}{\Delta Y} \frac{1}{f}
$$

where $\Delta t$ is the time step, $P$ the orbital time, and $Y$ is the normalized z-component of angular momentum given in Eq. (3.12).

After redistribution of orbits, due to collisions, those with $Y \leq Y_{\min }$ lie in the loss-cone, and the rest, out of it. Thus, accretion can be directly calculated using the fluxes $F_{Y}(X, Y)$ through the grid cell boundaries. Furthermore, due to the fact that orbits can scatter into 


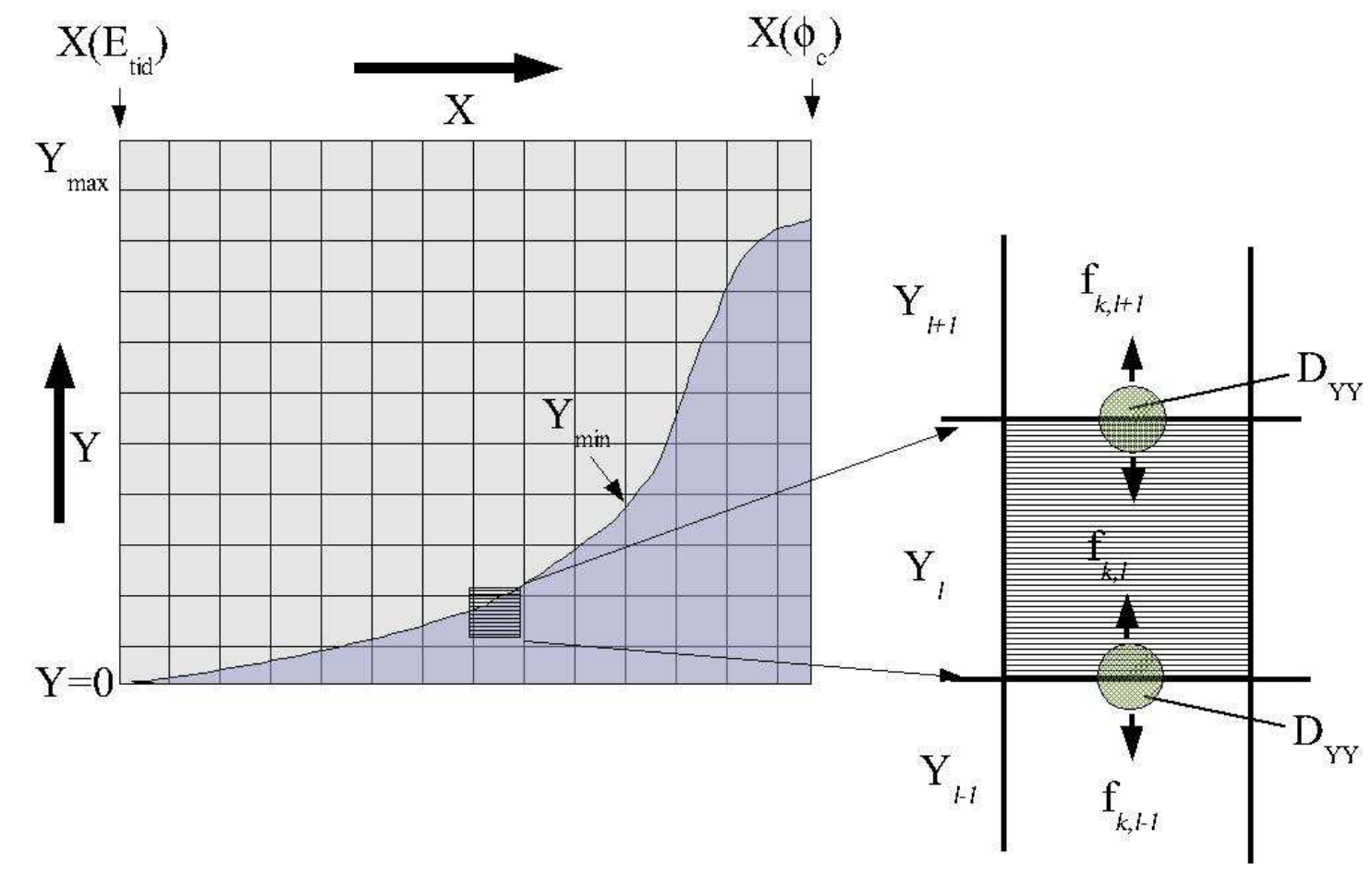

Figure 3.2: Schematic diagram of the numerical $X Y$-grid and definition of the loss cone. Half of the grid is shown in the left side of the figure. The other lower half corresponds to negative values of $\mathrm{Y}$ and is symmetric with respect to the $Y=0$-axis. The blue shaded area represents the loss cone in $X-Y$-space, and is limited by $Y_{\min }$. Stars are able to go into and out of it through angular momentum diffusion, as shown in the right side of the figure, for one grid cell. Energy diffusion to the loss cone in the horizontal direction is neglected, but they are able to escape the cluster through the tidal boundary at $X\left(E_{\mathrm{tid}}\right)$. 
the loss-cone or out of it before accretion depending on their crossing times, the contribution of $f(X, Y)$, of orbits belonging to the hole range of energies and angular momentum components, to the $\mathrm{BH}$ accretion given by the flux $F_{Y}$ should take into account this fact.

To this purpose, the ratio

$$
q \equiv \frac{t_{\mathrm{out}}}{t_{\mathrm{in}}} \propto \frac{\left(Y^{\mathrm{diff}}\right)^{2}}{\left(Y^{\mathrm{min}}\right)^{2}}
$$

of loss-cone depletion $\left(t_{\text {out }}\right)$ and replenishment $\left(t_{\text {in }}\right)$ time scales is defined (Lightman \& Shapiro, 1977). Here $Y^{\text {diff }}$ is the angular momentum diffusion term due to gravitational scattering per time step, given by the flux coefficient $D_{Y Y}$.

The ratio $q$ is used to transform angular momentum diffusion to $\mathrm{BH}$ star accretion. In other words, it permits to calculate the fraction of loss-cone scattered orbits through collisions, which are not be able to escape in an orbital time scale, and are thus accreted by the $\mathrm{BH}$, as well as the fraction of orbits outside the loss-cone, which can be eventually accreted.

If $q<1$, most loss-cone stars remain inside and $f(X, Y)$ is well represented by the flux of Eq. (3.26), but, because stars could be scattered out of the loss-cone to orbits to $Y>Y_{\min }$ in an orbital time scale, a correction to the angular momentum flux needs to be made (see next paragraph). In the classical approximation, accretion of stars inside $Y_{\min }$ leads to an 'empty loss-cone' $(f(X, Y)=0)$, but this is not a realistic boundary condition.

If $q>1$, the angular momentum diffusion is larger than the loss-cone opening, so that most stars manage to scatter out of the loss-cone and are not disrupted. The diffusion of orbits with $Y>Y_{\min }$ is corrected to accretion in a similar way as in the previous case, i.e. the 'full loss-cone' regime is not really full.

$q\left(E_{\text {crit }}\right)=1$, defines a critical energy $\left(E_{\text {crit }}\right)$ at a radius $r_{\text {crit }}$, in the equatorial plane, which marks the transition between the 'full' and 'empty' loss-cone regimes. Here the accretion is given exactly by Eq. (3.26), i.e. the angular momentum accretion flux $F_{\mathrm{Y}}^{\mathrm{acc}}=F_{\mathrm{Y}}$.

In order to illustrate the definition of $q$, consider the averaged angular momentum diffusion $\left\langle Y^{\text {diff }}\right\rangle$, at constant energy $X$. Thus, energy $X$ leads to a well defined $q(Y)$, and the critical energy is define as the energy $X$, where $\left\langle Y^{\text {diff }}\right\rangle=Y^{\text {min }}$. This is illustrated in Fig., (3.3), using data of a moderate concentrated $\left(W_{0}=6.0\right)$, high rotating model $\left(\omega_{0}=0.9\right)$, at time $t / t_{\mathrm{rh}_{\mathrm{i}}} \sim 3.4$. $E_{\text {crit }}$ is located in the intersection of both curves. As the $(X, Y)$ grid is two dimensional, $Y^{\text {diff }}$ could be smaller than $Y^{\text {min }}$, even at energies lower than $E_{\text {crit }}$, or $X_{\text {crit }}$, with some probability to fall into the loss cone. Thus, the mass flux into the loss cone need to take into account the values of $Y_{\min }, Y^{\text {diff }}$ and $X$ at each grid point, and $F_{Y}$ is corrected by the probability of accretion $P_{a}\left(X, Y, Y^{\text {diff }}\right)$, that a star do not escape from the loss-cone due to angular momentum diffusion once it is inside, or it can fall into the loss cone from outside. 


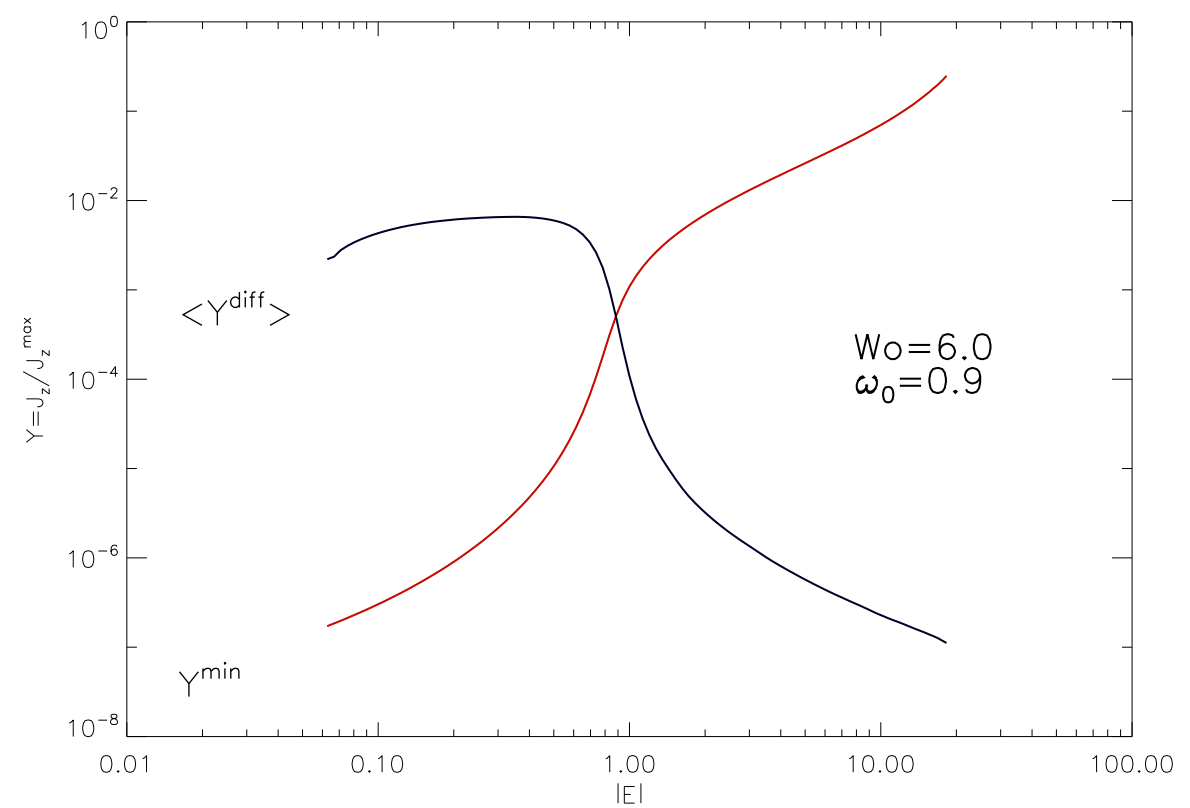

Figure 3.3: The panel shows the definition of critical energy $\left(E_{\text {crit }}\right)$ using data of a model with initial moderate concentration $W_{0}=6.0$ and high rotation $\omega_{0}=0.9$. The red curve is the losscone limit as defined in Eq. (3.24), and the black line, the averaged angular momentum diffusion at constant energy. The curves intersect at $\left(E_{\text {crit }}\right.$. 
Using the time scales of replenishment $t_{\text {in }} \propto\left(Y^{\text {min }}\right)^{2}$, and loss-cone depletion $t_{\text {out }} \propto$ $\left(Y^{\text {diff }}\right)^{2 *}$, the probability that a loss cone orbit $\left(Y<Y_{\min }\right)$ diffuse an amount $\Delta Y<$ $\left(Y-Y_{\min }\right)$ is given by:

$$
P_{a}(Y)=\int_{0}^{Y-Y^{\min }} \frac{2}{\sqrt{\pi} Y^{\text {diff }}} \exp \left(-\frac{Y^{\prime 2}}{\left(Y^{\text {diff }}\right)^{2}}\right) d Y^{\prime}
$$

i.e., centered at each $(X, Y)$ grid cell a Gaussian distribution of orbits in $Y$ with the dispersion $Y^{\text {diff }}$ is assumed. For orbits with $Y>Y_{\min }$, the probability that stars enter the loss cone is $1-P_{a}(X, Y)$, i.e. stars with $Y_{\text {diff }}$ diffuse an amount of $\Delta Y>\left(Y-Y_{\min }\right)$.

Finally, the fraction of orbits inside the loss cone, at each energy-angular momentum grid cell, which are accreted is given by:

$$
\left(\frac{\Delta f}{f}\right)_{\mathrm{acc}}=P_{a}(Y) \frac{\Delta t}{P} \frac{F_{\mathrm{Y}}}{\Delta Y} \frac{1}{f}
$$

for orbits outside the loss cone limit $\left(Y>Y_{\min }\right)$, this fraction is given by

$$
\left(\frac{\Delta f}{f}\right)_{\mathrm{acc}}=\left(1-P_{a}(Y)\right) \frac{\Delta t}{P} \frac{F_{\mathrm{Y}}}{\Delta Y} \frac{1}{f}
$$

and at the loss cone boundary $\left(Y=Y_{\min }\right)$ :

$$
\left(\frac{\Delta f}{f}\right)_{\text {acc }}=\frac{\Delta t}{P} \frac{F_{\mathrm{Y}}}{\Delta Y} \frac{1}{f}
$$

In order to get the total $\mathrm{BH}$ accretion mass, the distribution function $f\left(E, J_{\mathrm{z}}\right)$ is integrated in real and phase space, as:

$$
\Delta m_{a c c}=4 \pi \int_{0}^{\varpi^{\mathrm{tid}}} \varpi d \varpi \int_{0}^{z^{\mathrm{tid}}} d z\left[\frac{2 \pi}{\varpi} \int_{\phi_{c}}^{E_{\mathrm{tid}}} d E \int_{-J_{\mathrm{z}}^{\max }}^{+J_{\mathrm{z}}^{\max }} d J_{\mathrm{z}} \Delta f\left(E, J_{\mathrm{z})}\right]\right.
$$

where $\varpi^{\text {tid }}$ and $z^{\text {tid }}$ give the tidal cluster radius in $\varpi$ and $z$ directions respectively. Note that a factor of $2 \times 2 \pi=4 \pi$ is due to consideration of positive and negative zenithal coordinates (i.e. $\mathrm{z}$-direction) and that the azimuthal component is symmetric. $\pm J_{\mathrm{z}}^{\max }$ represents the maximum z-component of angular momentum in both directions, as $J_{\mathrm{z}}^{\max }= \pm \varpi \sqrt{2(E-\phi)}$.

The accretion mass is added to $M_{\mathrm{bh}}=M_{\mathrm{bh}}^{\text {old }}+\Delta m_{\mathrm{acc}}$ and furthermore $M_{\mathrm{cl}}=M_{\mathrm{cl}}^{\text {old }}-$ $\Delta m_{\text {acc }}$.

\subsubsection{Tidal limit}

A step to consider more realistic conditions in the evolution of globular clusters is made including a tidal limit originated by the potential field of a parent galaxy. A comparison to evolution of isolated clusters, using the non-BH version of the code is found in Kim et al.

\footnotetext{
${ }^{*}$ the square root dependence of $J_{\mathrm{z}}^{\text {min }}$ on time reflects the fact, that entry into the loss cone is a diffusive process
} 
$(2002)$.

The cluster is considered to orbit in the spherical potential of its parent galaxy at a constant distance (circular orbit) so that the mean density within the tidal radius $\left(r_{\text {tid }}\right)$ remains a constant throughout the evolution. An energy condition is used in order to remove stars from the cluster, i.e. stars, whose energy is greater than the initial tidal energy $\left(E_{\mathrm{tid}_{\mathrm{i}}}\right)$, are lost. $E_{\mathrm{tid}_{\mathrm{i}}}$ and $r_{\mathrm{tid}_{\mathrm{i}}}$ are calculated self-consistently in the initial models and adapted to the new cluster mass after diffusion through the tidal energy limit $X\left(E_{\text {tid }}\right)$ (see Fig. 3.2), each time step. The tidal radius at time $t$ then follows

$$
\frac{r_{\mathrm{tid}}(t)^{3}}{r_{\mathrm{G}}^{3}}=\frac{M_{\mathrm{cl}}(t)}{M_{\mathrm{G}}}
$$

where the galactrocentric distance $r_{\mathrm{G}}$ and the mass of the galaxy $M_{\mathrm{G}}$ are constants throughout the evolution.

The tidal energy at any time is given by $E_{\text {tid }}(t)=G M(t) / r_{\text {tid }}(t)$, then the fraction over the initial tidal energy becomes

$$
\frac{E_{\mathrm{tid}}(t)}{E_{\mathrm{tid}}(0)}=\frac{M_{\mathrm{cl}}(t) t_{\mathrm{tid}_{\mathrm{i}}}}{r_{\mathrm{tid}}(t)}=M(t)^{2 / 3}(t)
$$

due to the fact, that $M_{\mathrm{cl}_{\mathrm{i}}} \equiv 1$. In this way $E_{\text {tid }}$ can be advanced each time step to the new cluster mass. The well known theoretically observed development of radial anisotropy, specially after the core collapse phase, is a good indicator for the strongness of the tidal criteria, in the sense that radial anisotropy should decrease after collapse due to rapid loss of radial orbits, but it will be stronger if the tidal field is weak.

\subsubsection{Ellipticity}

As already reported from theoretical studies by Shapiro \& Marchant (1976) and observations (of LMC clusters) by Frenk \& Fall (1982), an age-ellipticity relation in globular clusters exists, in the sense, that old globular clusters are more spherical than younger ones. Flattening can be originated by either anisotropy in the velocity dispersion or by rotation.

Goodman (1983) derives an expression to calculate the dynamical ellipticity of a system in equilibrium, defined as the non-projected system ellipticity with the same amounts of kinetic energy in and out of the meridional plane, but with its density stratified on similar spheroids. It is for oblate spheroids (not triaxial):

$$
\frac{2 T_{\mathrm{rot}}+3 T_{\sigma_{\phi}}-T_{\sigma}}{T_{\sigma}-T_{\sigma_{\phi}}}=\frac{\left(1-2 s^{2}\right) \arccos s-3 s \sqrt{1-s^{2}}}{s \sqrt{1-s^{2}}-s^{2} \arccos s}
$$

where The axial ratio $s \equiv b / a=1-\mathrm{e}_{\mathrm{dyn}}$ is the solution of Eq. (3.36). $T_{\text {rot }}$ is the rotational energy, $T_{\sigma_{\phi}}$ is the energy contained in the azimuthal component of the velocity dispersion and $T_{\sigma}$ is the energy of all components of the velocity dispersion.

Ellipticity can be considered as one of the main basic evolutionary parameters in globular clusters (together with cluster concentration and age of the cluster ), and a good indicator 
of rotation in the evolution of cluster shapes, useful to obtain data from observations and compare it with theoretical models.

\subsection{Numerical method}

\subsubsection{Fokker-Planck step and loss-cone diffusion}

As seen in Eqs. 3.1 and 3.2, the solution of the Fokker-Planck equation makes necessary to define a distribution function in the integration of the diffusion coefficients (background distribution of stars), and one for the factors of the diffusion coefficients (distribution of test stars). Actually one should use the same function in both cases (as they describe the same stars), but this would rise the computational time proportional to $N_{\varpi} \times N_{\mathrm{z}} \times N_{\mathrm{X}}^{2} \times N_{\mathrm{Y}}^{2}$, where $N_{\mathrm{i}}$ are the number of grid cells in real and velocity space. Thus, an approximation of the background distribution function is used in Fokker-Planck studies, which reduces this complexity to a time proportional to $N_{\varpi} \times N_{\mathrm{z}} \times N_{\mathrm{X}} \times N_{\mathrm{Y}}$. This approximation makes use of a rotating Maxwellian velocity distribution to the background:

$$
f_{\mathrm{b}}(\vec{v})=\frac{n_{f}}{\left(2 \pi \sigma_{f}^{2}\right)^{3 / 2}} \exp -\frac{\left(\vec{v}-\Omega_{f} \varpi \overrightarrow{e_{\varphi}}\right)^{2}}{2 \sigma_{f}^{2}}
$$

where $n_{f}, \Omega_{f}$ and $\sigma_{f}$ correspond to the zeroth, first and second order moments of the distribution function, and are the density, angular velocity and one-dimensional velocity dispersion of the field star distribution, respectively.

The Fokker-Planck equation in discretized form, is solved in the $X, Y$-grid, where the distribution function $f_{\mathrm{k}, \mathrm{l}}=f\left(X_{\mathrm{k}}, Y_{\mathrm{l}}\right)$ is defined in the center of each grid cell of size $\Delta X$ and $\Delta Y$, as well as $\Omega_{f}, \sigma_{f}, n_{f}$. The flux coefficients $F_{\mathrm{i}}$ are calculated at the cell boundaries through a logarithmic interpolation of the respective $f_{\mathrm{k}, 1}$ 's. The finite difference scheme of the Fokker-Planck equation takes the form:

$$
p_{\mathrm{k}, \mathrm{l}}^{\prime} \cdot \frac{\left(f_{\mathrm{k}, \mathrm{l}}-f_{\mathrm{k}, \mathrm{l}}^{\mathrm{old}}\right)}{\Delta t}=-\frac{F_{\mathrm{X}_{\mathrm{k}+1 / 2,1}}-F_{\mathrm{X}_{\mathrm{k}-1 / 2,1}}}{\Delta X}-\frac{F_{\mathrm{Y}_{\mathrm{k}, 1+1 / 2}}-F_{\mathrm{Y}_{\mathrm{k}, 1-1 / 2}}}{\Delta Y}
$$

where $p_{\mathrm{k}, 1}^{\prime}$ is the discretized phase space volume as calculated in equation (3.15). $f_{\mathrm{k}, 1}^{\text {old }}$ gives the distribution function at the last time step and $f_{\mathrm{k}, \mathrm{l}}$, its value at the current time step.

The discretized system of equations is solved implicitly using a sparse matrix method (Henyey et al. 1959) borrowed from a gaseous model code (Spurzem 1994, 1996). The Henyey3 theory is used, where the direct neighborhood of the cells in $X$ and $Y$ directions is use to calculate the new distribution function. This method gives the equation matrix the special form of a main diagonal and two sub-diagonals. Only two iterations are necessary to achieve convergence of the solution. Furthermore, a Chang-Cooper scheme is applied to the energy direction in order to improve conservation characteristics (Chang \& Cooper 1970).

The new distribution $f(X, Y)$ due to stellar orbit scattering is computed in the diffusion step (FP step). Then, accretion is calculated as described in Sect. (3.2.2). The mass flux of accretion, $\Delta m_{\mathrm{acc}}$, is added to $M_{\mathrm{bh}}$, which leads to a new BH-potential $\phi_{\mathrm{bh}}^{\text {new }}=\phi_{\mathrm{bh}}+G M_{\mathrm{bh}} / r$. 
A new $X, Y$-grid need to be build due to the change in the potential. The $\mathrm{BH}$ influence radius $\left(r_{\mathrm{a}}\right)$ is re-calculated as well as the critical radius $r_{\text {crit }}$, corresponding to the new critical energy $E_{\text {crit }}=\phi\left(r_{\text {crit }}\right)+J_{\mathrm{z}}^{\max } / 2 r^{2}$ using a Newton-Raphson scheme. At the same time, the size of the loss cone needs to be re-defined with the new loss-cone limit $J_{\mathrm{z}}^{\min }$. As a consequence of the small modification of the potential, the shape of stellar orbits evolve while conserving adiabatic invariants, i.e. the z-component of angular momentum and adiabatic invariant $q\left(E, J_{\mathrm{z}}\right)$ (see below). Finally, $f(X, Y)$ is re-calculated through interpolation to the new $(X, Y)$-grid corresponding to the new potential using a second-order bivariate Taylor expansion for each mess point (see next paragraph), and correspondingly, the density profile of stars is re-calculated after integration of the number density of stars.

\subsubsection{Vlasov Step and calculation of the cluster potential}

After diffusion and accretion have taken place, the cluster potential has to be advanced. The density is calculated from the new distribution function according to

$$
n(\varpi, z)=\frac{2 \pi}{\varpi} \int_{\phi_{c}}^{E_{\mathrm{tid}}} \int_{-\varpi \sqrt{2(E-\phi)}}^{-\varpi \sqrt{2(E-\phi)}} f\left(E, J_{\mathrm{z}}\right) d E d J_{\mathrm{z}}
$$

The Poisson equation is solved and a new cluster potential is calculated, following,

$$
\nabla^{2} \phi(\varpi, z)=4 \pi n(\varpi, z) ; \quad(G \equiv 1)
$$

The system of equations is solved with the same sparse matrix method (Henyey et al. 1959) as for the diffusion step, thereby taking account of the even sparser matrices occurring due to the Poisson equation.

It makes necessary a readjustment of the distribution function (due to slow adiabatic potential changes), caused by a redistribution of energies in the system, but under conservation of angular momentum and adiabatic invariant $q\left(E, J_{z}\right)$, defined as

$$
q\left(E, J_{\mathrm{z}}\right)=8 \pi^{2} \iint_{A\left(E, J_{z}\right)}\left(v_{\varpi}^{2}+v_{z}^{2}\right) d \varpi d z
$$

note that the integrand is equal to $2(E-\phi)-\left(J_{\mathrm{z}}^{2} / \varpi^{2}\right)$ and evaluates to zero on the boundary of $A\left(E, J_{\mathrm{z}}\right)$. Goodman (1983) emphasizes that $q$ is the axially symmetric analogue of the radial action

$$
Q(E, J)=2 \iint_{r_{-}}^{r^{+}} v_{r} d r
$$

in a spherically symmetric potential, and actually comprises the average of the radial action over $J$ with boundaries $J_{\mathrm{z}}$ and $J_{\max }$, if the potential is spherically symmetric, thus representing an average of an adiabatic invariant with respect to the third integral. Then, the orbit-averaged Boltzmann equation takes the form

$$
\frac{\partial f}{\partial t}+\frac{1}{p} \frac{\partial q}{\partial t} \frac{\partial f}{\partial E}=\left(\frac{\partial f}{\partial t}\right)_{\text {coll }}
$$


what indicates, that, neglecting any encounters,

$$
\left.\frac{\partial f}{\partial t}\right|_{q, J_{z}}=0
$$

To this purpose, a new $\left(E, J_{\mathrm{z}}\right)$ grid is created, due to the energy changes and the new $J_{\mathrm{z}}^{\max }(E)$. Furthermore, the meridional plane is rastered to determine the area $A\left(E, J_{\mathrm{z}}\right)$ and the adiabatic invariant $q\left(E, J_{\mathrm{z}}\right)$ is numerically integrated. It is required in order to fulfil the conservation of $f\left(q, J_{z}\right)$. At first, the new $(E, J z)$ grid is transformed uniquely into a $\left(q, J_{\mathrm{z}}\right)$ grid. A second-order bivariate Taylor expansion is then performed for each mesh point in the new grid to derive approximate values from the known values of $f\left(q^{\prime}, J_{\mathrm{z}}^{\prime}\right)$ in the old grid, i.e. $f\left(E, J_{\mathrm{z}}\right)$ is allowed to change. This again gives a new density distribution $n$ in Eq. (3.39) and the procedure is repeated until convergence is reached. In doing this the first determined $f\left(q, J_{\mathrm{z}}\right)$ is retained as the old grid in order to save accuracy. Nevertheless, this second-order interpolation step turns out to be, relatively, the most important source of numerical error in the code. An error in the calculation of the distribution function at maximum z-component of angular momentum was found (lower extremes in the $\left.f(E)\right|_{J_{z}}$ curves in Fig. 4.1), but this deviations do not influence the BH accretion, which dominates at $J_{\mathrm{z}} \leq J_{\mathrm{z}}^{\mathrm{min}}$.

Goodman (1983) reports a test of the influence of the third integral on the procedure just stated. He relaxed an initial King model distribution function (non-rotating) violently in assigning to it a Plummer potential. The result was a strong dynamical collapse with an increase in $n_{\mathrm{c}}$ of a factor of 56 . The collapse generated a flattening $e=0.055$, which is attributed to the third integral, because there is no preferential axis of rotation or anisotropy initially that could flatten the system. On the other hand, the test is very crude, because as Goodman himself states the above procedure is only first-order in energy conservation between the initial and final values of $\phi$ and the collapse picture is not appropriate to be described by adiabatic invariants. Although the effect of diffusion is to wipe out the (here artificially) generated azimuthal anisotropy, the flattening of non-rotating, isotropic configurations during their evolution is a more appropriate measure of the influence of the third integral.

\subsection{Resume}

The Fokker-Planck method used for this study allows a detailed analysis of energy and angular momentum transport in a self-consistent way. The system evolves in relaxation time scales, before it dissolves in the galaxy tidal field. Evolution is driven by relaxation due to small angle scatterings $(\Delta v / v \ll 1)$, computed as velocity diffusion coefficients up to second order. There is no correlation between collisions (like in three body collisions), which could be important for the energy generation in the core that can reverse the collapse. In fact the $\mathrm{BH}$ potential represents an energy source in the system.

The loss cone problem, is fully treated using angular momentum diffusion. A star is disrupted if its z-component of angular momentum is less than a minimum angular momentum $J_{\mathrm{z}}^{\mathrm{min}}$. A 'seed' initial $\mathrm{BH}$ is calculated using a first small perturbation of the system. Its mass is initially very small in comparison to $M_{\mathrm{Cl}_{\mathrm{i}}}$ and it grows slowly, through accretion 
of stars in mainly low- $J_{\mathrm{z}}$ (radial) orbits, leading to a growing central potential cusp. Stellar distribution around the central $\mathrm{BH}$ is assumed to be represented by a single-particle system that is initially axisymmetric in real space and anisotropic in velocity space. No stellar spectrum is included in the model.

In the present study, non-ergodicity on the hypersurface (given by $E$ and $J_{z}$ ) is neglected due to any third integral $I_{3}$. Furthermore, the potential close to the BH is spherically symmetric $(\sim 1 / r)$, and $I_{3}$ could be fairly approximated by $J^{2}$, specially at later evolutionary times, when the BH potential cusp dominates. Implications of this constraint are discussed also in the next chapters. Furthermore, the cluster is considered to orbit in the spherical potential of its parent galaxy at a constant distance (circular orbit) so that the mean density within the tidal radius $\left(r_{\text {tid }}\right)$ remains a constant throughout the evolution.

The present work has mainly following aims:

- To study evolutionary states of initially rotating globular clusters, with embedded star accreting black holes and to follow the behavior of evolution indicators as accretion rates, angular momentum distribution and shape of the clusters, as well as evaluate the importance of the onset of gravo-gyro vs. gravo-thermal instabilities. Following questions are aimed to be answered:

- are gravo-gyro effects uncoupled from gravo-thermal evolution?

- can a BH reverse collapse faster than processes like binary heating?

- how faster becomes the cluster a spherical shape in comparison with models without $\mathrm{BH}$ ?

- To define final steady states of systems with BH and determine if the presence of a $\mathrm{BH}$ in an initially flattened rotating system has an observable effect on its final state of evolution, so that it deviates from that of the well-known spherical case (density cusp, dispersions, mass loss). Following questions are aimed to be answered:

- how GCs behave in comparison to other stellar systems with BH like galactic nuclei? Does BH accretion stalls?

- Are final steady state solutions applicable to GCs?

- to define criteria that permits to infer from the properties of presently observed clusters, using detailed observational data (with higher core resolution), information about the pre BH cluster. Following questions are aimed to be answered:

- is rotation a good descriptor in all evolutionary states?

- are spherical models in this sense sufficient? 


\section{Chapter 4}

\section{Results}

Evolution of a set of models of rotating star clusters with embedded black holes is shown in this section. Starting point is the description of initial conditions using rotating King models (Sect. 4.1), and following with the evolution of cluster structure of representative selected models (Sect. 4.2), which will be used for comparison of evolutionary behavior using different rotation and concentrations. The well studied spherically symmetric system of clusters of stars with BH is used as test and comparison in this section. Section (4.3) emphasizes on evolution of rotation (rotational velocity, angular momentum) and the influence of cluster relaxation and black hole accretion on it. Redistribution of 2-dimensional number density of stars (distribution function) in energy-angular momentum space is treated in Sect. (4.4). Applications in comparison with observations are presented in Sect. (4.5).

A comparison of the results between models with and without rotation and with or without $\mathrm{BH}$ is made. The behavior of non-rotating models is followed, in order to check the pure effect of rotation in the simulations and compare with previous spherically symmetric studies of GCs with BH. Analysis of models using the non-BH version of rotating models is important due to the necessity of criteria, that could elucidate the existence of BHs in globular star clusters from observations, as mentioned in Sect. (2.3). BH mass limits and mass growth rates are given and compared with observational studies. Furthermore, questions formulated in Sect. (3.4) and further implications are discussed. 


\subsection{Initial conditions}

The existence of 'seed' BHs in globular star clusters is still not a fully answered question, as discussed in Sects. (1.4) and (2.3). Moreover, initial models with embedded BHs can be constructed starting with the existence of a small initial BH situated in the cluster center (its possible origin was discussed in Sect. 1.4).

As initial cluster configurations, truncated King models with added bulk motion are used. This velocity field takes the form of a Maxwellian distribution such that the mean velocity scales in proportion to radius near the center, and falls off outside. Their adopted distribution function is

$$
\begin{array}{ll}
f\left(E, J_{z}\right) \propto \exp \left(-\beta \Omega_{0} J_{z}\right) \cdot\left[\exp \left(-\beta\left(E-E_{\mathrm{tid}}\right)\right)-1\right], & E<E_{\mathrm{tid}} \\
0 \quad, \quad & E>E_{\mathrm{tid}}
\end{array}
$$

where the inverse square central velocity dispersion is given by $\beta=1 / \sigma_{\mathrm{c}}^{2}$ and $\Omega_{0}$ is an angular velocity. In figure (4.1), $f\left(J_{z}\right)$ at constant energy $E$ against $J_{z}$, for an initial high rotating model $\left(W_{0}=0.6, \omega_{0}=0.9\right)$, is shown. $f\left(J_{z}\right)$ covers a wide range of values in logarithmic scale, and $J_{\mathrm{z}}$ varies from negative to positive values, accordingly to two directions of rotation around the z-axis. The values of $f$ at $J_{z}=0$ represent initially number density of stars on radial orbits, while the extreme $J_{\mathrm{z}}^{\max }$, the circular orbits. Note that the angular velocity $\Omega_{0}$ is given by the slope of $f$ in each curve of constant energy, which becomes shorter the larger is the absolute value of the energy $|E|$ (leading to smaller $\left|J_{\mathrm{z}}^{\max }\right|$ ). The range of energies plotted is listed on the left column in the panel.

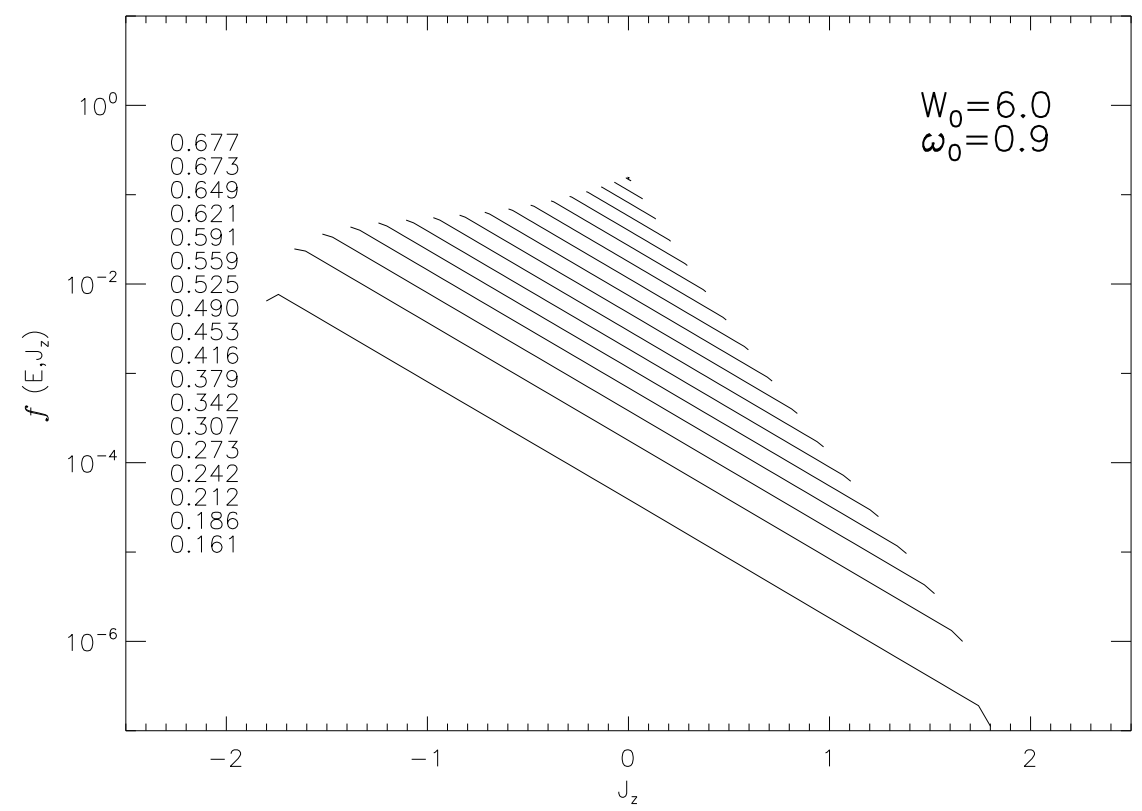

Figure 4.1: $f\left(J_{z}\right)$, at constant energy $E$, for an initial model $\left(W_{0}=0.6, \omega_{0}=0.9\right)$. From top to bottom the curves have smaller $|E|$, listed on the left column. Grid dimension is (100 61) in $\left(E, J_{\mathrm{z}}\right)$-space used to construct the models. 
The initial conditions of each model are fixed by the triple $\left(W_{0}, \omega_{0}, M_{\mathrm{bh}_{\mathrm{i}}}\right)$, see Table (4.1). Here, $W_{0}$ is the familiar King parameter

$$
W_{0}=\frac{\left(\phi\left(r_{\text {tid }}\right)-\phi_{\mathrm{c}}\right)}{\sigma_{\mathrm{c}}^{2}} ; \quad(\text { King 1966, Spitzer 1987) }
$$

where $\phi\left(r_{\text {tid }}\right)$ is the potential at the cluster tidal boundary, due to the potential field of its parent galaxy, and $\phi_{\mathrm{c}}$ the central cluster potential.

$\omega_{0}$ is the initial rotational parameter:

$$
\omega_{0}=\sqrt{9 /\left(4 \pi G n_{\mathrm{c}}\right)} \Omega_{0}
$$

where $G$ is the gravitational constant and $n_{\mathrm{c}}$ the central cluster density.

\begin{tabular}{|c|c|c|c|}
\hline Model name & $W_{0}$ & $\omega_{0}$ & $M_{\mathrm{bh}_{\mathrm{i}}} / M_{\mathrm{cl}_{\mathrm{i}}}$ \\
\hline \hline $\mathrm{A} 1$ & 3.0 & 0.00 & $5 \cdot 10^{-6}$ \\
\hline $\mathrm{A} 2$ & 3.0 & 0.30 & $5 \cdot 10^{-6}$ \\
\hline $\mathrm{A} 3$ & 3.0 & 0.60 & $5 \cdot 10^{-6}$ \\
\hline $\mathrm{A} 4$ & 3.0 & 0.90 & $5 \cdot 10^{-6}$ \\
\hline A5 & 3.0 & 1.20 & $5 \cdot 10^{-6}$ \\
\hline B1 & 6.0 & 0.00 & $5 \cdot 10^{-6}$ \\
\hline B2 & 6.0 & 0.30 & $5 \cdot 10^{-6}$ \\
\hline B3 & 6.0 & 0.60 & $5 \cdot 10^{-6}$ \\
\hline B4 & 6.0 & 0.90 & $5 \cdot 10^{-6}$ \\
\hline B5 & 6.0 & 1.20 & $5 \cdot 10^{-6}$ \\
\hline C1 & 9.0 & 0.20 & $5 \cdot 10^{-6}$ \\
\hline C2 & 9.0 & 0.30 & $5 \cdot 10^{-6}$ \\
\hline C3 & 9.0 & 0.40 & $5 \cdot 10^{-6}$ \\
\hline C4 & 9.0 & 0.50 & $5 \cdot 10^{-6}$ \\
\hline
\end{tabular}

Table 4.1: Initial models used in the simulations. The initial parameters are in Column 1: King potential; Column 2: dimensionless rotation and Column 3: initial BH mass in $M_{\mathrm{cl}}$-units

Radii are given in units of the initial cluster core radius $\left(r_{\mathrm{c}_{\mathrm{i}}} \equiv 1\right)$. All units can be rescaled to physical units by fixing initial mass and core radius of the cluster. Applications to globular clusters and comparison with observations for the non-BH case and using a similar parameter set can be found in Fiestas \& Spurzem (2005). Use of a large set of initial configurations allows a properly identification of evolved clusters from observational studies by identifying evolutionary cluster parameters as concentration and cluster ellipticity, together with the age of the cluster, reducing in this way the number of model identifications after following the evolution backwards in time. Table (4.2) shows the initial parameters of the models used.

Intermediate $W_{0}=6.0$ models are expected to approximately reproduce current evolutionary states of globular star clusters. Their initial concentrations given by $\ln \left(r_{\text {tid }} / r_{\mathrm{c}_{\mathrm{i}}}\right)$ 
decrease and their dynamical ellipticities increase, the higher the initial rotation, as a response of the rotational origin of cluster flattening. $W_{0}=3.0$ models show relatively large cores as seen in the low rate of $\ln \left(r_{\mathrm{h}} / r_{\mathrm{c}_{\mathrm{i}}}\right)$ in Table (4.2). Their half-mass relaxation times are very close to each other nearly independent of initial rotation, but their initial ellipticity grows with $\omega_{0}$, as well as the rotational energy. High concentrate models $\left(W_{0}=9.0\right)$ are known to be unstable against gravothermal collapse (Katz, 1980) due to their strong isothermal character. Their cores are small as seen in their tidal radii, which are around 30 times larger than the respective core radii, and the half-mass radii, which are between 6 and 10 times larger than the core radii. The smallest cores are present in the non-rotating models, like in all other models. The ellipticities are higher in comparison to the less concentrated models with the same rotational parameter, as well as the rate of rotational to kinetic energy.

\begin{tabular}{|c|c|c|c|c|c|c|}
\hline Model name & $\ln r_{\text {tid }} / r_{\mathrm{c}_{\mathrm{i}}}$ & $\ln r_{\mathrm{h}} / r_{\mathrm{c}_{\mathrm{i}}}$ & $e_{\text {dyn }}$ & $t_{\mathrm{rc}_{\mathrm{i}}}$ & $t_{\mathrm{rh}_{\mathrm{i}}}$ & $T_{\text {rot }} / T_{\text {kin }}$ \\
\hline \hline $\mathrm{A} 1$ & 1.76 & 0.44 & -0.001 & 20.886 & 30.80 & 0.00 \\
\hline $\mathrm{A} 2$ & 1.74 & 0.43 & 0.015 & 20.882 & 30.65 & 1.07 \\
\hline $\mathrm{A} 3$ & 1.69 & 0.39 & 0.059 & 20.876 & 28.93 & 4.08 \\
\hline $\mathrm{A} 4$ & 1.51 & 0.38 & 0.195 & 20.878 & 28.36 & 13.84 \\
\hline A5 & 1.51 & 0.32 & 0.192 & 20.900 & 25.95 & 13.66 \\
\hline B1 & 2.92 & 0.99 & -0.001 & 19.24 & 91.88 & 0.00 \\
\hline B2 & 2.71 & 0.96 & 0.105 & 19.21 & 87.73 & 7.00 \\
\hline B3 & 2.29 & 0.87 & 0.278 & 19.40 & 76.32 & 19.81 \\
\hline B4 & 1.92 & 0.83 & 0.403 & 19.86 & 71.24 & 30.25 \\
\hline B5 & 1.57 & 0.82 & 0.500 & 20.48 & 71.28 & 39.85 \\
\hline C1 & 3.68 & 2.36 & 0.365 & 10.98 & 741.8 & 26.87 \\
\hline C2 & 3.23 & 2.12 & 0.465 & 11.62 & 524.8 & 35.45 \\
\hline C3 & 2.91 & 1.95 & 0.497 & 12.29 & 406.9 & 40.89 \\
\hline C4 & 2.65 & 1.84 & 0.501 & 12.95 & 341.8 & 44.41 \\
\hline
\end{tabular}

Table 4.2: Parameters of initial models used in the simulations. Column 1: model identification name; Column 2: concentration; Column 3: $\ln \left(r_{\mathrm{h}} / r_{\mathrm{c}_{\mathrm{i}}}\right)$; Column 4: dynamical ellipticity; Column 5: initial central relaxation time in code units; Column 6: initial half-mass relaxation time in code units; Column 7: rate of rotational energy to kinetic energy.

$M_{\mathrm{bh}_{\mathrm{i}}}$ is the initial black hole mass, in units of the initial cluster mass $M_{\mathrm{cl}_{\mathrm{i}}}$, latter is set to 1. Numerical tests were made to define appropriate 'seed' initial BH-masses, according to small changes in the central potential in order to evolve the stellar distribution function and thus the structural parameters (density, dispersions, rotation) in a self-consistent way. This criteria takes into account a deviation of an initially rotating King model into a state of dynamical equilibrium under adiabatic changes of the potential. $M_{\mathrm{bh}_{\mathrm{i}}}=5 \cdot 10^{-6} M_{\mathrm{cl}_{\mathrm{i}}}$ was chosen as the best solution to start with, without the necessity of constructing new distribution functions or density profiles to the system including the new BH potential. Moreover, for a typical massive globular cluster with an initial mass of five million stellar components, in a single solar-mass model, this approximation leads to an initially BH-mass of $25 M_{\odot}$, as a seed IMBH. In this sense, $M_{\mathrm{bh}_{\mathrm{i}}}$ constitutes an upper limit for the initial change in the King potential, and at the same time guarantees the set off of angular momentum diffusion towards the central BH. Table (4.3) shows the initial BH parameters for all models. The initial accretion radii are rather small compared to the core radii, specially 
for the $W_{0}=3.0$ models, for which the core radii are larger. Initial relaxation times at $r_{\mathrm{a}}$ in the latter models are also longer than in the other models, as the density is smaller here, and the velocity dispersion higher. On the other hand, the fraction of the initial $\mathrm{BH}$ potential (which absolute value is the same for all models, as the initial BH mass is constant) to the maximal energy of orbits is higher for the most concentrated models due to the higher energies in this models while this fraction for the lower concentrated models is higher.

\begin{tabular}{|c|c|c|c|}
\hline Model name & $\ln \left(r_{\mathrm{a}} / r_{\mathrm{c}_{\mathrm{i}}}\right)$ & $t_{\text {rha }}$ & $\phi_{\mathrm{BH}_{\mathrm{c}}} / E_{\max }$ \\
\hline \hline $\mathrm{A} 1$ & -4.00 & 1.429 & 3.33 \\
\hline $\mathrm{A} 2$ & -4.00 & 1.725 & 3.32 \\
\hline $\mathrm{A} 3$ & -4.00 & 1.724 & 3.29 \\
\hline $\mathrm{A} 4$ & -4.00 & 1.724 & 3.23 \\
\hline $\mathrm{A} 5$ & -4.00 & 1.725 & 3.15 \\
\hline $\mathrm{B} 1$ & -3.61 & 1.327 & 6.06 \\
\hline $\mathrm{B} 2$ & -3.60 & 1.401 & 5.94 \\
\hline $\mathrm{B} 3$ & -3.61 & 1.411 & 5.53 \\
\hline B4 & -3.62 & 1.443 & 4.98 \\
\hline $\mathrm{B} 5$ & -3.64 & 1.491 & 4.42 \\
\hline $\mathrm{C} 1$ & -3.17 & 0.763 & 13.95 \\
\hline $\mathrm{C} 2$ & -3.21 & 0.808 & 12.10 \\
\hline C3 & -3.24 & 0.855 & 10.57 \\
\hline C4 & -3.27 & 0.901 & 9.34 \\
\hline
\end{tabular}

Table 4.3: Initial model parameters related to initial $\mathrm{BH}\left(M_{\mathrm{bh}_{\mathrm{i}}}=5 \cdot 10^{-6} M_{\mathrm{cl}_{\mathrm{i}}}\right)$ used in the simulations. Column 1: rate of $\mathrm{BH}$ influence radius to core radius; Column 2: relaxation time at influence radius and Column 3: rate of initial central $\mathrm{BH}$-potential to maximal energy

In the following, a parametric description of the evolution of the system (density, dispersions, rotation) is presented. Intermediate models $\left(W_{0}=6.0\right)$ are used as a starting point to compare with the set of models $W_{0}=3.0$ and $W_{0}=9.0$ using different initial rotational parameters.

\subsection{Evolution of cluster structure}

Starting with the described initial configurations, the initial BH mass $\left(M_{\mathrm{BH}}\right)$ starts growing through accretion of stars in low angular momentum orbits. As $M_{\mathrm{BH}}$ grows, central density increases while the $\mathrm{BH}$-potential $\left(\sim G M_{\mathrm{BH}} / r\right)$ dominates the stellar distribution within the $\mathrm{BH}$ radius of influence $r_{\mathrm{a}}$, which grows itself in time with $M_{\mathrm{BH}}$. As the system evolves, it loses mass through the outer tidal boundary due to relaxation effects and through $\mathrm{BH}$ star accretion under the effects of a redistribution of orbits driven by the growing potential cusp. Evolution of the density profile for models A3 and B3 (same rotation, different concentrations) is shown in Figure (4.2). The extent of the BH gravitational influence is noted on each curve at the position of the influence radii $r_{\mathrm{a}}$ (squares in the curves). Evolutionary profiles are represented by dot-dashed curves at the given times (in parenthesis). Note that one can roughly approximate the division between the cusp and the core at $\sim r_{\mathrm{a}}$ at any time of evolution. The central cusp in the density profile grows first very slow and accelerates as 
the cluster is closer to collapse.

The final steady state is the sloped solid curve in Figs. (4.2) and (4.3), which evolve in all cases towards a power-law of $\lambda=-1.75$, according to $n \propto r^{\lambda}$. This steady-state solution has been extensively studied in the spherical case by Bahcall \& Wolf (1976), Lightman \& Shapiro (1977), Marchant \& Shapiro (1980) and others. It forms inside $r_{\mathrm{a}}$ and is maintained in the post-collapse phase, when the evolution is driven through energy input from the central object. Very close to the center the density profile flattens due to the effective loss-cone accretion and, at the other extreme in the halo, it gets steeper beyond $r_{\mathrm{a}}$ up to the tidal radius of the galaxy $\left(r_{\text {tid }}\right)$, where the loss-cone loses its significance. $r_{\text {tid }}$ itself becomes smaller in time as a consequence of tidal mass loss. In all cases the halo cusp is steeper than $\lambda=-3.5$ (e.g. Spitzer \& Shapiro, 1972). The cusp of the halo is flattened in time, as can be seen in the figures, but as the influence radius of the $\mathrm{BH}$ increases, the central cusp $(\lambda=-1.75)$ extends and dominates zones always closer to the halo. Furthermore, the cluster evolves into the post-collapse phase, which begins earlier for higher initially concentrated clusters, and re-expands later until cluster dissolution.

Regarding evolution of the density profile, the comparison of the models presented is qualitative similar, despite of the collapse times ( $t_{\mathrm{cc}}$ is shorter for the high rotating models), after which the density drops. Observe a slightly shrink of the influence radius at earlier evolutionary times, as the radius where $M_{\mathrm{cl}}=M_{\mathrm{bh}}$ becomes slightly smaller, caused mainly by core contraction, driven by gravogyro and gravothermal instabilities towards collapse. Afterwards, BH mass growth dominates the determination of influence radius. Note that the core radius is initially larger than $r_{\mathrm{a}}$ and shrinks during core collapse, while $r_{\mathrm{a}}$ grows with $M_{\mathrm{bh}}$, mainly at times close to collapse up to $\sim r_{\mathrm{c}_{\mathrm{i}}} \equiv 1$. In Fig (4.3) the evolution of density profile for the model $W_{0}=9.0$ with initial rotation $\omega_{0}=0.3$ is shown. The collapse time in this models are generally shorter than in the less concentrated previous ones due to a high initial concentration, but all models approximate the $\lambda=-1.75$ cusp, independent of concentration and rotation.

A more detailed view of the evolution of density is obtained by its distribution in the meridional plane $(\varpi, z)$, as shown in Fig.(4.4) for different times in units of the initial halfmass relaxation time. In models without $\mathrm{BH}$ one expects a density growth of the core, as the system evolves towards collapse. The initially King profile would, nevertheless, maintain a flattened core up to the collapse, with a higher slope of the halo profile, beyond the core radius. This would lead to nearly constant isodensity contours towards the center. In the regions where $\mathrm{BH}$ star accretion dominates (i.e. inside $r_{\mathrm{a}}$ ), the isodensity contours grow due to the presence of the BH (lighter contours in Fig. 4.4). The cusp can be noted in the colour gradient towards the center. It forms very fast in a short time close to core collapse. At the same time, the system loses mass through the outer tidal boundary, and the cluster tidal radius becomes smaller (darker areas in the contour plots). Particularly, one can note at later times the higher density of stars in $\varpi$-direction in the center than in $z$-direction in this models (Fig. 4.4d). This suggests that the system achieves to a concentration of stars in the equatorial plane, very close to the $\mathrm{BH}$, than in the direction of the axis of rotation. The nature of this bounded stable orbits is discussed below and in the next section. Moreover, $\mathrm{BH}$ accretion seems to be more efficient in the zones closer to the $z$-axis, and the zones along the $\varpi$-axis close to the BH will be finally more populated (see also Fig. 4.21d later, 

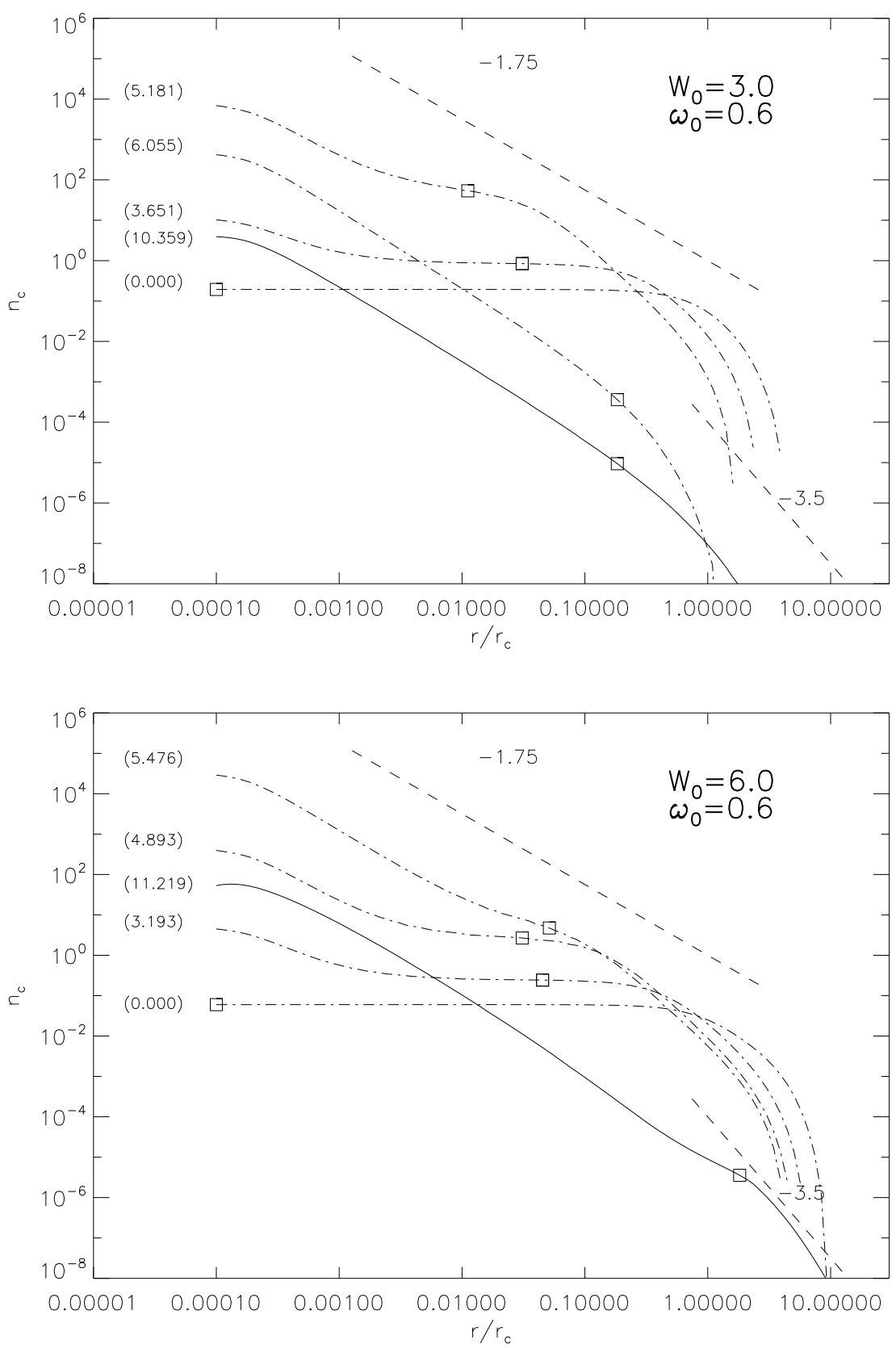

Figure 4.2: Equatorial density profile $(z=0)$ for models $W_{0}=6.0$ and $W_{0}=3.0$, and initial rotation $\omega_{0}=0.6$ (Models A3 and B3) for different times (in parenthesis), given in units of initial half-mass relaxation time $\left(t_{\text {rhi }}\right)$. The dashed line shows the $-7 / 4$ slope and the dot-dashed lines the evolutionary profiles. The location of $r_{\mathrm{a}}$ is shown as squares. The BH mass is initially $5 \cdot 10^{-6}$ and $M_{\mathrm{bh}} \sim 0.01 M_{\mathrm{cl}}(0)$ as the curve achieves the steady state (solid sloped line). 


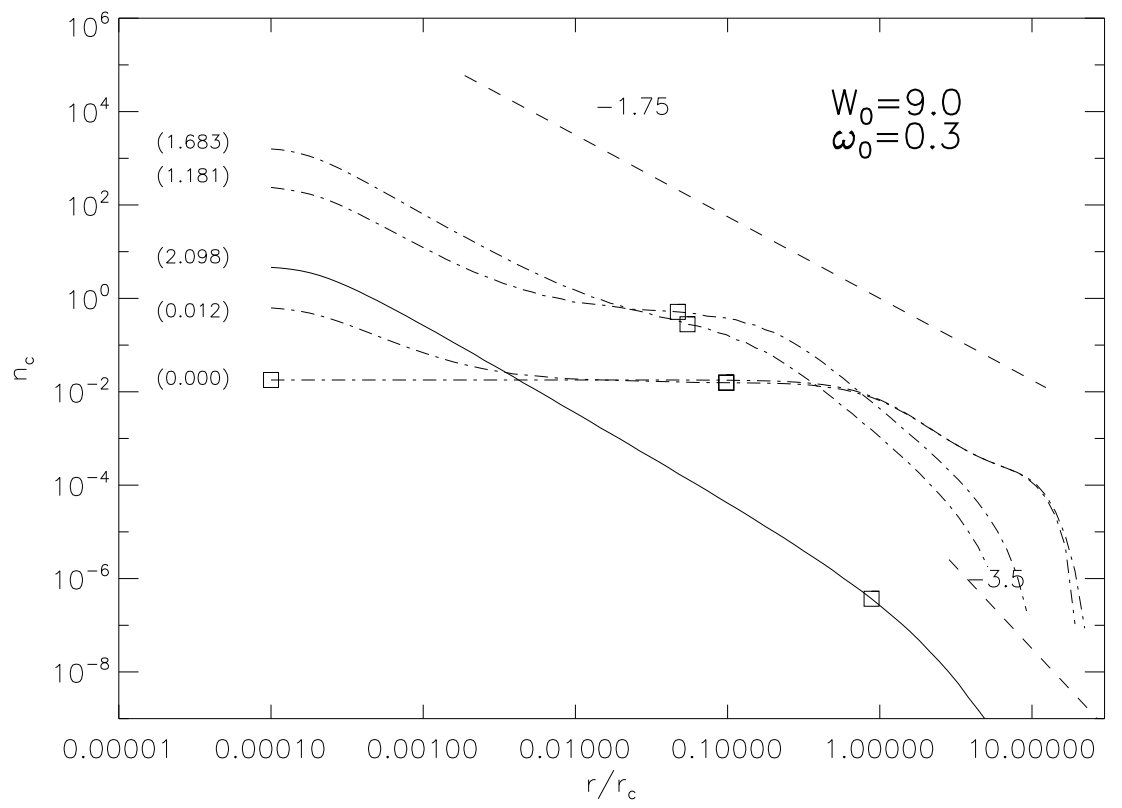

Figure 4.3: Same as Fig. 4.2 for models of $W_{0}=9.0$ and initial rotation $\omega_{0}=0.3$ (Model C2). 
for comparison and further discussion in Sect. 4.3).

Here should be noted, that circular meridional orbits could not be distinguished from radial orbits, due to the neglection of the third integral, and so have a larger probability of being accreted by the BH. This is not the case for the circular equatorial orbits, where they are well represented by their $J_{\mathrm{z}}^{\max }$, and are not affected in their distribution due to this effect. Here $J^{2}$ would be a good approximation due to the sphericity of the potential close to the BH. Moreover, the neglection of this constant of motion, could in some sense exaggerate the accretion rate in the $\mathrm{z}$-direction.

As the systems evolves, orbits in the region of influence of the BH gain kinetic energy as they become Keplerian bounded. Their velocity dispersion experiences a growth in a similar way as the density does, towards the center. The dispersion approximates a power-law of $-1 / 2$ within the $\mathrm{BH}$ influence radius $r_{\mathrm{a}}$ (i.e. motion of stars inside $r_{\mathrm{a}}$ are dominated by the gravitational force of the central mass). Figs. (4.5) and (4.6) show the evolution of the total one-dimensional velocity dispersion profile in the same way as Figs. (4.2) and (4.3) do for the density. The velocity dispersion grows significantly inside $r_{\mathrm{a}}$ and rapidly when the cluster is close to the collapse (evolution is shown as dot-shaded lines and positions of $r_{\mathrm{a}}$ as squares). The figures suggest that, although rotation tends to order the motion of stars, dispersion increases inside $r_{\mathrm{a}}$ up to collapse time, due to the presence of the central $\mathrm{BH}$. Evolutionary times in this figures are chosen the same as in the density profiles, in order to compare the acceleration of evolution close to the collapse. Note that central velocity dispersion remains increasing as the density begins to fall after collapse is reversed and core expansion sets off. On the other hand, the total dispersion in the halo falls rapidly as the $\mathrm{BH}$ influence radius continues growing.

Furthermore, the final profiles of tangential and radial components of velocity dispersion $\left(\sigma_{\phi} \neq \sigma_{r}\right)$ are not identical, as expected for anisotropy. Fig. (4.7) shows the total 1 -D velocity dispersion $\sigma_{t}$ together with its components, where $\sigma_{t}^{2}=\sigma_{\phi}^{2}+2 \sigma_{r}^{2}$. Radial and zenithal components are equivalent in the models $\left(\sigma_{r}=\sigma_{z}\right)$. Note that the dispersion becomes tangentially biased in the core and radially biased in the halo, what confirms previous studies of anisotropy distribution in the center of stellar systems with BH (Quinlan et al, 1995; Freitag \& Benz 2002). Fig. (4.8) shows the radial distribution of anisotropy in the system at different times. Anisotropy is defined as $A \equiv 1-\frac{\sigma_{\phi}{ }^{2}}{\sigma_{\mathrm{r}}{ }^{2}}$. The initial profile shows a maximum positive halo anisotropy (radial orbits dominate the halo in the initial configuration). Negative (tangential) anisotropy forms first slowly inside the BH influence radius. This process accelerates at collapse time, while radial anisotropy forms in the halo after a depletion in the initial times. After collapse, tangential orbits dominate the system $(A<0)$.

Figure (4.9) shows the evolution of central density for the model $W_{0}=0.6$ and rotation parameters $\omega_{0}=0.0$ (non-rotating), $0.3,0.6,0.9$ and 1.2. Time is given in units of initial half mass relaxation time $\left(t_{\mathrm{rh}_{\mathrm{i}}}\right)$. After a similar initial evolution of all models, collapse happens always in a shorter time, the higher the initial rotation (it needs less than two relaxation time units in the highest rotating model presented here, $\omega_{0}=1.2$ ). The collapse phase seems to be strongly influenced by gravogyro instabilities in the models with the fastest initially rotation as it is gravothermal in the initially non-rotating models $\left(\omega_{0}=0.0\right)$. In the intermediate rotating models, gravothermal and gravogyro instabilities drive collapse, 
a) $t / t_{r h i}=0.00$

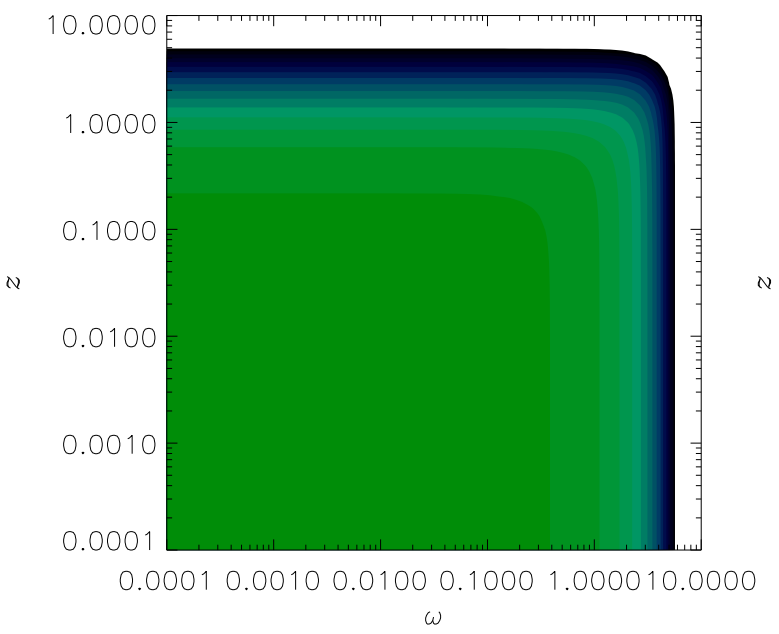

c) $t / t_{\text {rhi }}=3.46$

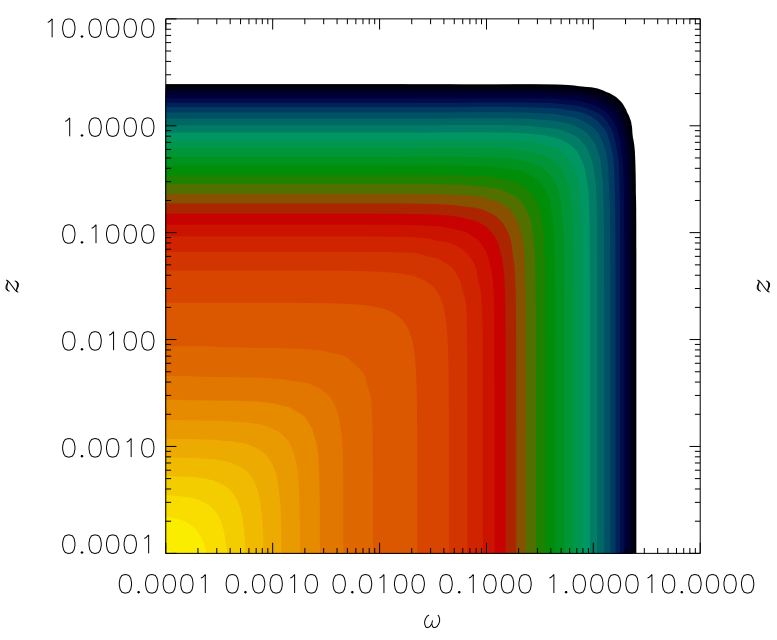

b) $t / t_{\text {rhi }}=2.60$

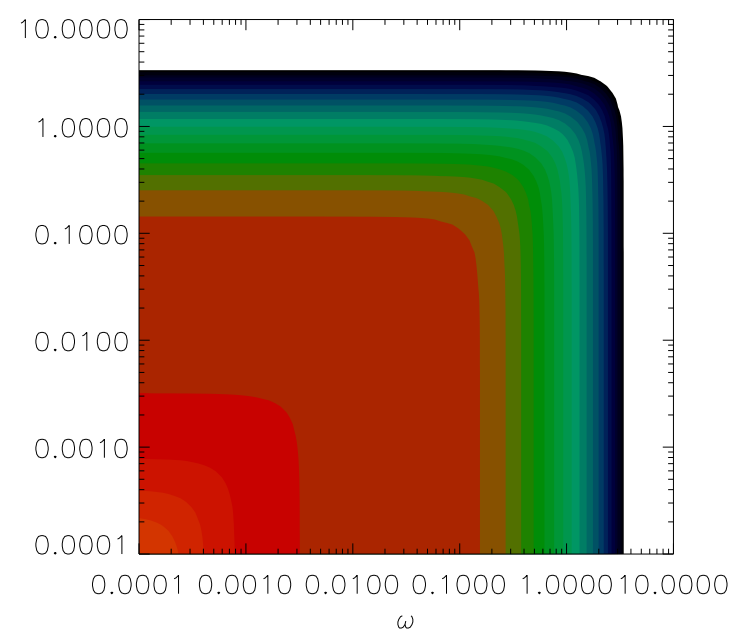

d) $t / t_{\text {rhi }}=5.43$

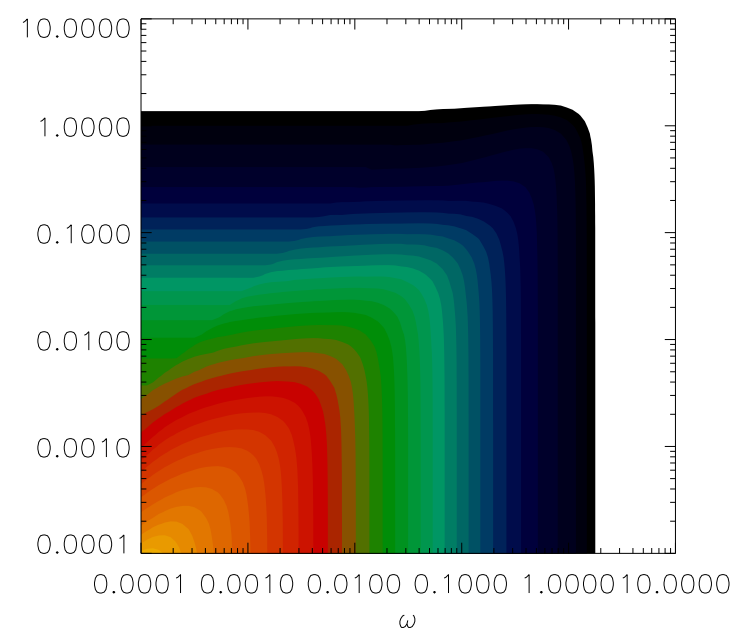

Figure 4.4: Evolution of density distribution in the meridional plane for a model B4. Double logarithmic cylindrical coordinates $(\varpi, z)$ are used. Lighter zones represent higher isodensity contours. The time is given in units of initial half-mass relaxation time $\left(t_{\mathrm{rhi}}\right)$. 

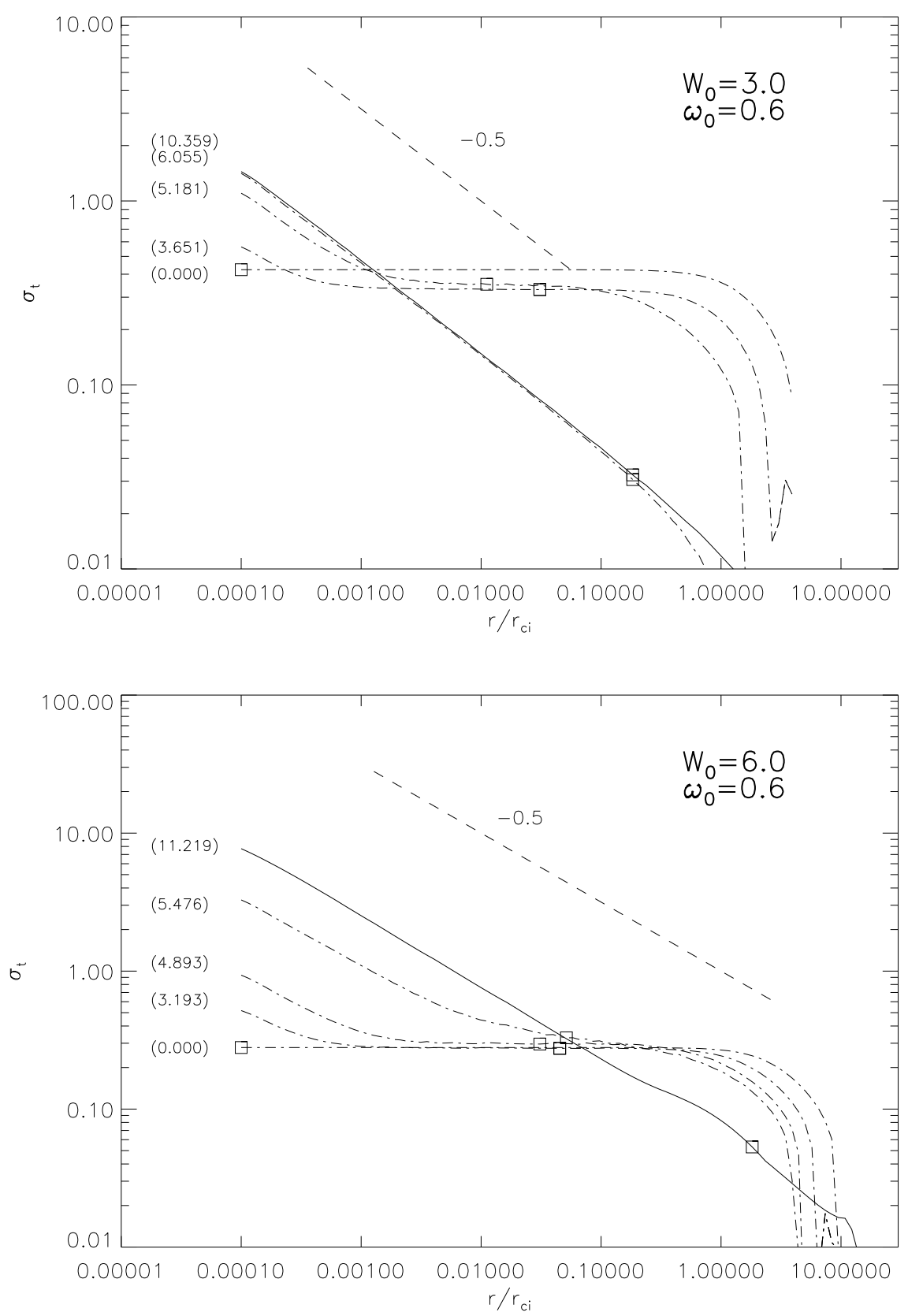

Figure 4.5: Equatorial profile $(z=0)$ of $1 \mathrm{D}$ total velocity dispersion as in Figures (4.2) and (4.3) for the density (Models A3 and B3). The dashed line shows the $-1 / 2$ slope and the dot-dashed lines the evolutionary profiles. The location of $r_{\mathrm{a}}$ is shown as squares. The curve achieves the steady state (solid sloped line) close to the collapse. 


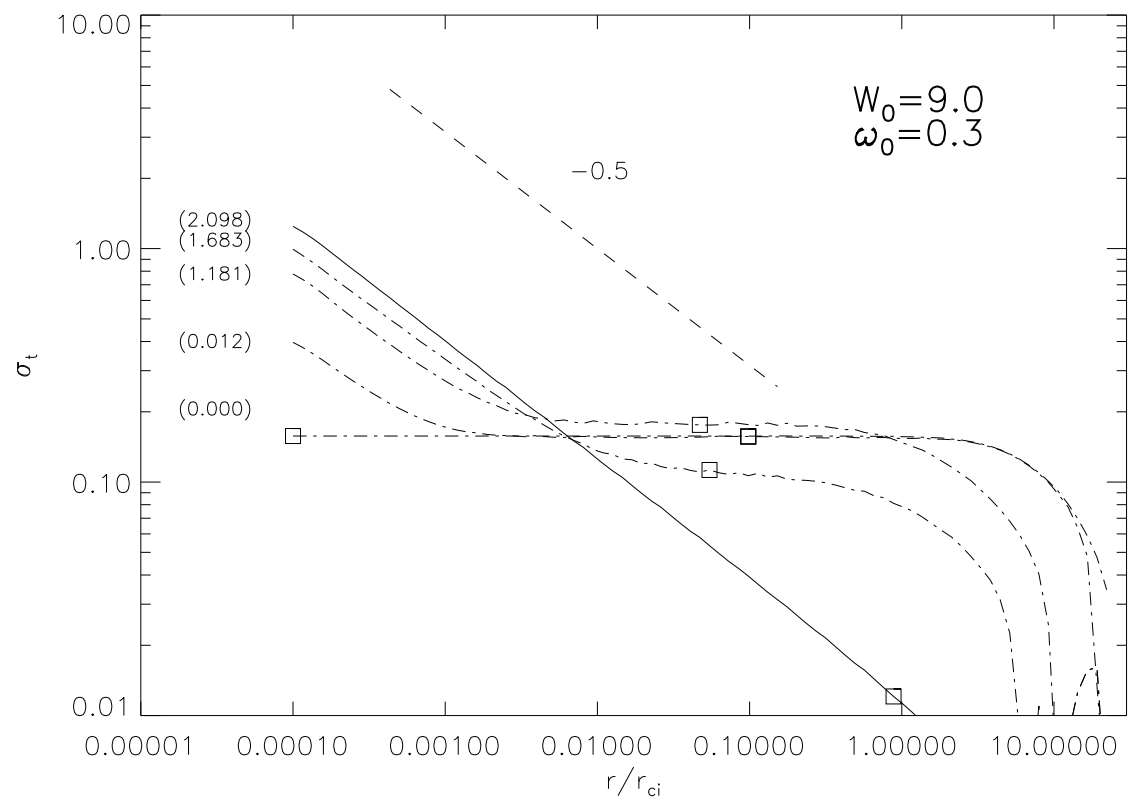

Figure 4.6: Same as Fig. 4.5, for the model $W_{0}=9.0$ and initial rotation $\omega_{0}=0.3$ (Model C2). Note the faster evolution and lower final dispersions in the center as in the less concentrated models. 


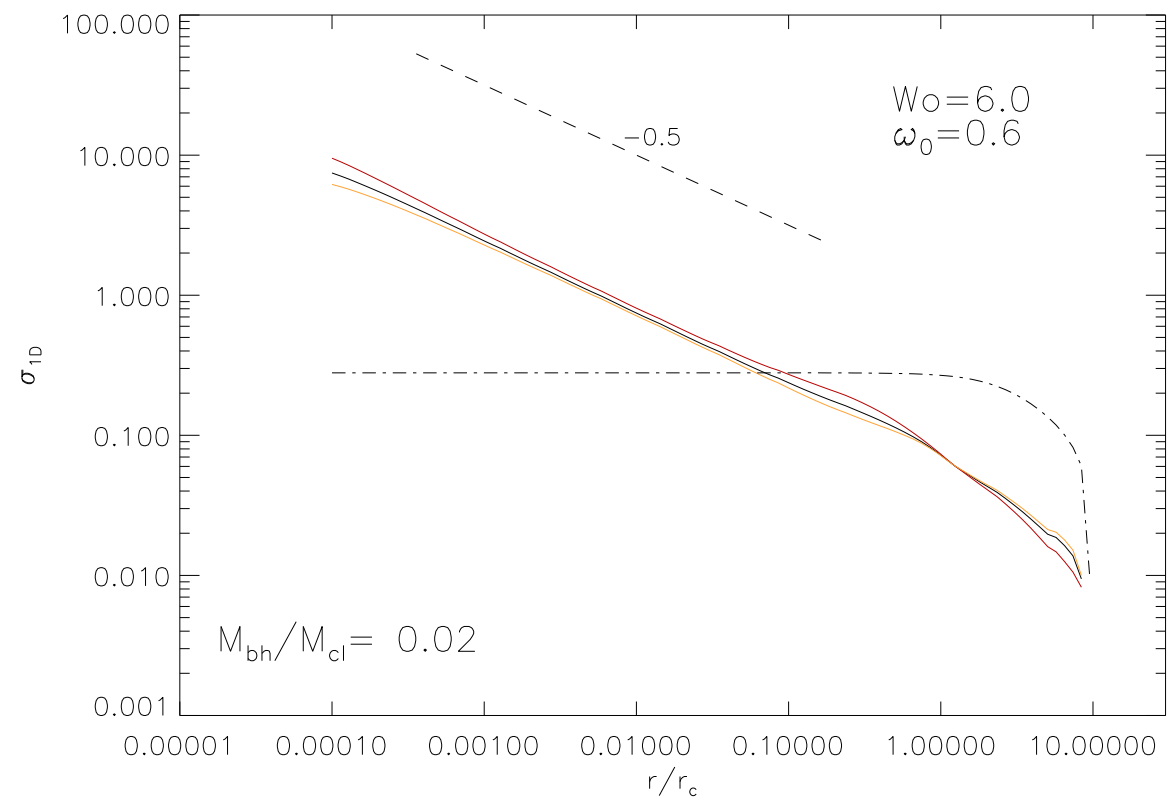

Figure 4.7: 1D total velocity dispersion for the final state of model of Fig. 4.5. The dashed line shows the $-1 / 2$ slope and the dot-dashed line the initial total 1D dispersion profile. Note the higher tangential component $\left(\sigma_{\phi}\right)$ in the central regime (red line) and the lower radial component $\left(\sigma_{r}\right)$ due to $\mathrm{BH}$ accretion of low- $J_{z}$ radial orbits (yellow line).

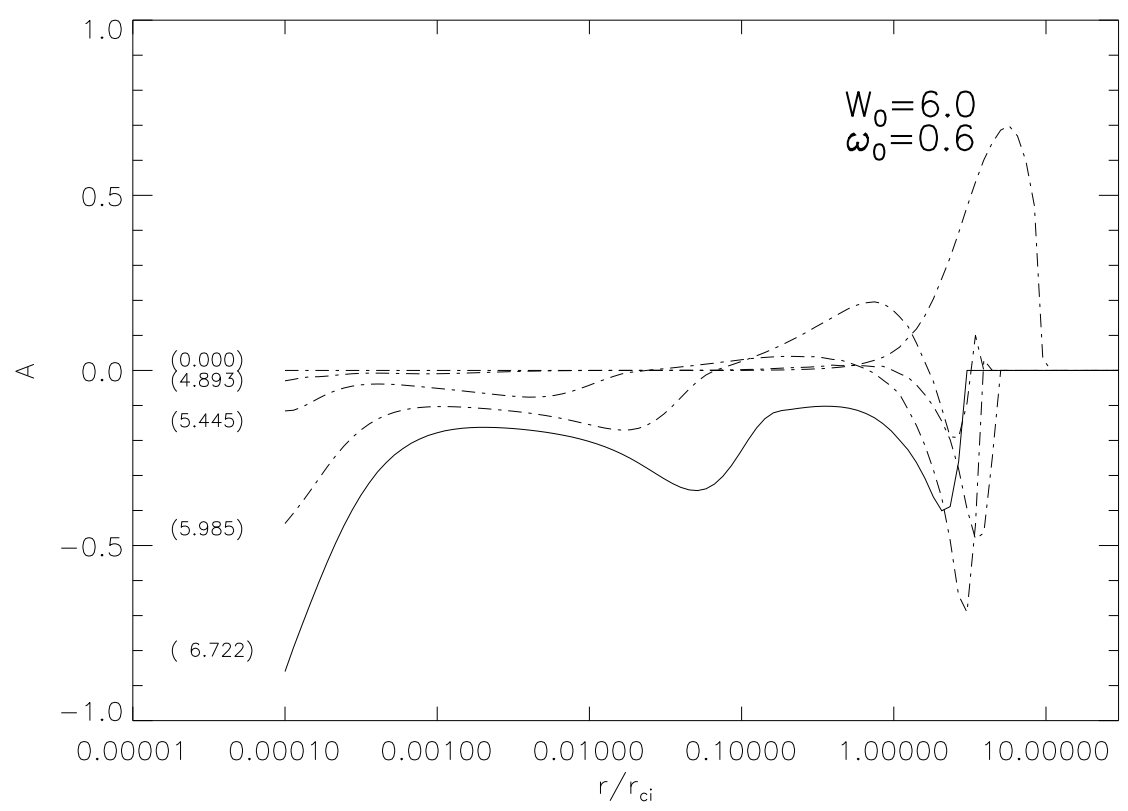

Figure 4.8: Anisotropy, defined as $A \equiv 1-\frac{\sigma_{\phi}{ }^{2}}{\sigma_{\mathrm{r}^{2}}}$, against radius for the same model of Fig. 4.7. The dashed line represents the zero-line and the dot-dashed line the initial profile. 
while the angular momentum is driven out of the cluster in an always more efficient way for the higher rotating models. A purely gravogyro or gravothermal phase is generally not observed, as the initial gravogyro catastrophe observed by Akiyama \& Sugimoto (1989) in their N-Body calculations. Both instabilities seem to occur together and support each other. The collapse phase is reversed due to the energy source built by the star accreting $\mathrm{BH}$, while the central density drops. From a comparison to non-BH models, a further acceleration of collapse seems to happen due to the presence of the BH. It acts, as an energy source, through which energetic stellar orbits are formed, which are easily accreted by the $\mathrm{BH}$. In this way, mass is lost through $\mathrm{BH}$ accretion, although with an initially lower rate. As angular momentum diffusion drives tidal mass loss of initially circular pro-grade halo orbits increasing the radial anisotropy in the halo, the probability of BH star accretion of low- $J_{\mathrm{z}}$ orbits increases. Thus, tidal mass loss supports $\mathrm{BH}$ star accretion and leads to an increasingly $\mathrm{BH}$ mass growth rate during core collapse.

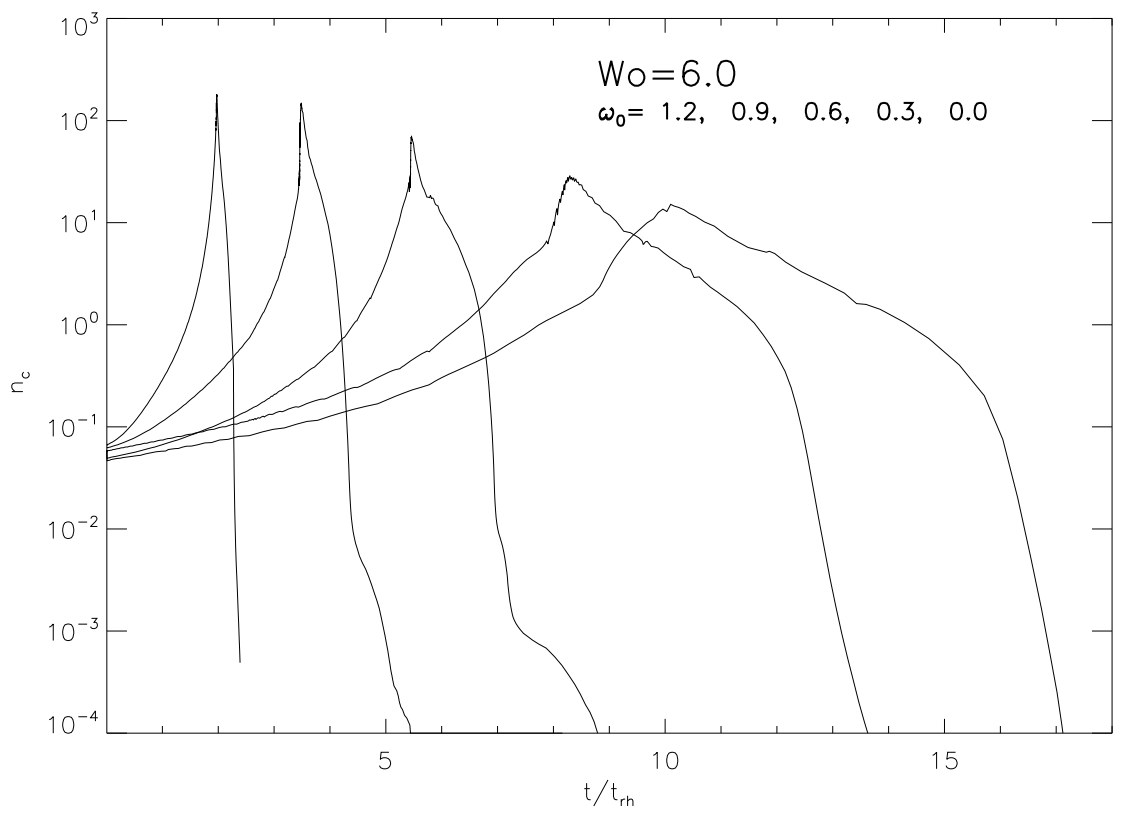

Figure 4.9: Evolution of central density for models of $W_{0}=6.0$ and initial rotation $\omega_{0}=0.0,0.3,0.6,0.9,1.2$. The time is given in units of initial half-mass relaxation time $\left(t_{\mathrm{rhi}}\right)$.

A comparison to the models $W_{0}=3.0$ for different rotation parameters (Fig. 4.10) shows a rapid increase in the central density in a similar way depending on the initial rotation. Core heating and angular momentum transport seem to be here more effective, reversing collapse always earlier and at lower density maxima, the higher the rotation. A higher rotation accelerates also cluster mass loss through the galactic tidal boundary and supports $\mathrm{BH}$ star accretion in a similar way as previously described, but in a shorter time scale. As mentioned, these models start with larger cores, and generally lower ellipticities.

Direct influence of the central $\mathrm{BH}$ in the redistribution of orbits, for models with same initial conditions, can be seen in Table (4.4), where collapse parameters of rotating BH and non-BH models are compared. The acceleration of collapse time in models with $\mathrm{BH}$ is 


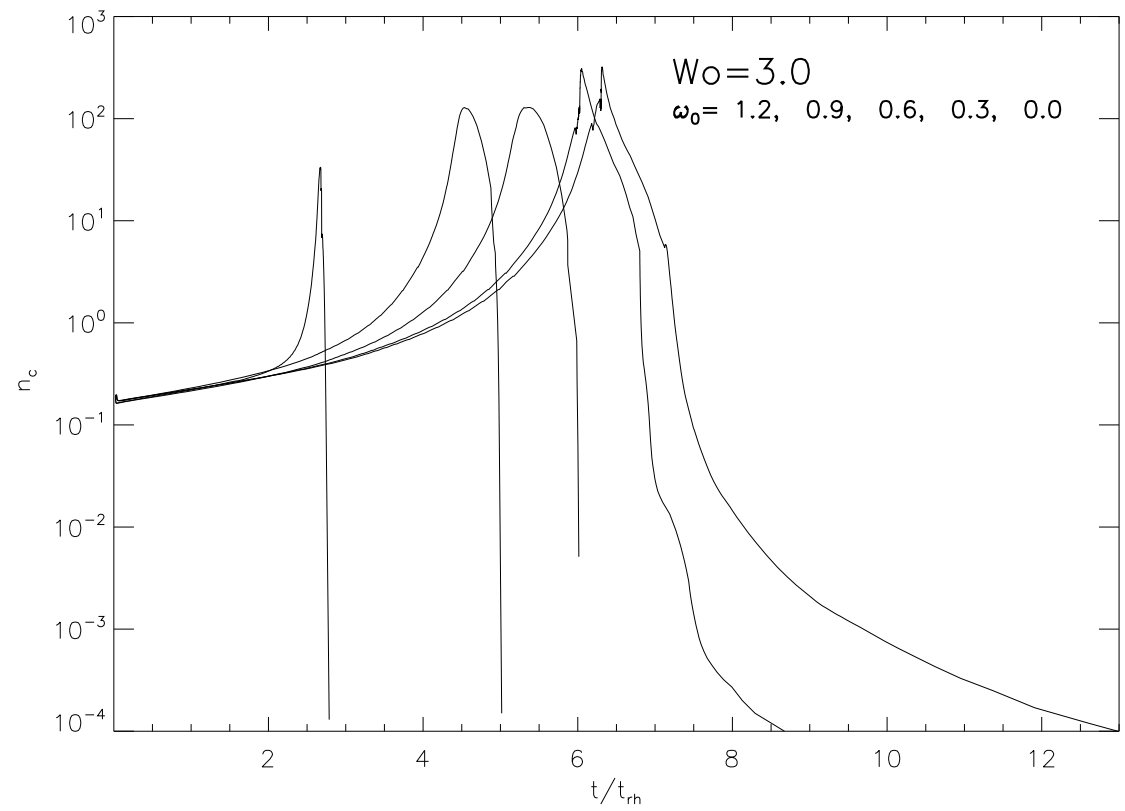

Figure 4.10: Evolution of central density for models of $W_{0}=3.0$ and initial rotation $\omega_{0}=0.0,0.3,0.6,0.9,1.2$. The time is given in units of initial half-mass relaxation time $\left(t_{\mathrm{rhi}}\right)$. 


\begin{tabular}{|c||c|c|c||c|c|c|}
\hline \multirow{2}{*}{\multicolumn{1}{|c||}{ Model name }} & \multicolumn{3}{c||}{ non-BH models } & \multicolumn{3}{c|}{ BH models } \\
\cline { 2 - 7 } & $t_{\mathrm{cc}} / t_{r h_{i}}$ & $M_{\mathrm{cc}}$ & $t_{50} / t_{r h_{i}}$ & $t_{\mathrm{cc}} / t_{r h_{i}}$ & $M_{\mathrm{cc}}$ & $t_{50} / t_{r h_{i}}$ \\
\hline \hline A1 & 8.70 & 0.25 & 5.85 & 7.05 & 0.15 & 4.25 \\
\hline A2 & 8.39 & 0.24 & 5.43 & 6.05 & 0.12 & 3.23 \\
\hline A3 & 7.85 & 0.22 & 4.80 & 5.56 & 0.07 & 2.56 \\
\hline A4 & 6.78 & 0.19 & 3.71 & 4.58 & 0.06 & 2.13 \\
\hline A5 & 5.80 & 0.15 & 2.80 & 2.69 & 0.01 & 1.28 \\
\hline B1 & 11.80 & 0.60 & 13.20 & 10.21 & 0.37 & 8.23 \\
\hline B2 & 10.46 & 0.48 & 10.10 & 8.42 & 0.33 & 6.51 \\
\hline B3 & 7.24 & 0.33 & 5.40 & 5.46 & 0.19 & 3.04 \\
\hline B4 & 4.85 & 0.23 & 2.60 & 3.47 & 0.16 & 1.64 \\
\hline
\end{tabular}

Table 4.4: Comparison of collapse parameters between rotating $\mathrm{BH}$ and non-BH models. $t_{\mathrm{cc}}$ : core collapse time

$t_{50}$ : time at which the cluster lose half of its mass

$M_{\mathrm{cc}}$ : current cluster mass at $t \approx t_{\mathrm{cc}}$

evident in all cases, which is higher for the initially faster rotating clusters. It is slightly higher for the $W_{0}=3.0$ models (20 to $50 \%$ faster with increasing rotation) in comparison to the $W_{0}=6.0$ models (13 to $30 \%$ faster with increasing rotation). The highest acceleration of collapse is observed in the high rotating, low concentrated model A5 $\left(3.0,1.2,5 \cdot 10^{-6}\right)$. Since BH accretion of stars in low- $J_{\mathrm{z}}$ orbits leads to an ordered motion in the region inside $r_{\mathrm{a}}$, it generates an angular momentum source, which supports the transport of $J_{\mathrm{z}}$ through relaxation to the outer parts (gravogyro effect) and accelerates the collapse. At the same time, increasing of high energetic radial orbits leads to a higher accretion rate specially close to the collapse time. Thus, smaller collapse times are driven by the mutual support of angular momentum transport and BH star accretion.

As seen in Fig. (4.11) for the model B4 $\left(0.6,0.9,5 \cdot 10^{-5}\right)$, after a small increase at early times, the growth of the $\mathrm{BH}$ influence radius $\left(r_{\mathrm{a}}\right)$, where $M_{\mathrm{cl}}\left(r<r_{\mathrm{a}}\right)=M_{\mathrm{BH}}$, is dominated by core contraction, being most of the time smaller than the core radius, while $r_{\mathrm{c}}$ drops due to core collapse. As it will be discussed later, $\mathrm{BH}$ mass accretion rate is initially small. At collapse time, while the $\mathrm{BH}$ mass growth rate increases, $r_{\mathrm{a}}$ grows faster than $r_{\mathrm{c}}$ towards the post-collapse phase. $r_{\mathrm{a}}$ grows further during post-collapse although $M_{\mathrm{BH}}$ stalls and $d M_{\mathrm{BH}} / d t$ drops, due to cluster mass loss through the galactic tidal boundary. See Fig. (4.18) for comparison. $r_{\mathrm{a}}$ rises above the half-mass radius and approaches the tidal radius at later evolutionary times.

Evolution of Lagrangian radii are a good indicator for the contraction and further reexpansion of mass shells. Their calculation in axisymmetric systems is, nevertheless, not straightforward, because one should define radii of mass spheroids rather than mass spheres. Nonetheless, for not strong flattened systems, as the ones treated here, Lagrangian radii can be considered to show deviations from spherical symmetry only up to second order in a Legendre expansion. They can be calculated at a zenithal angle, where the effects of probable flattening on the mass columns are not strong, i.e. $P_{2}(\cos \theta)=0$. This condition leads 


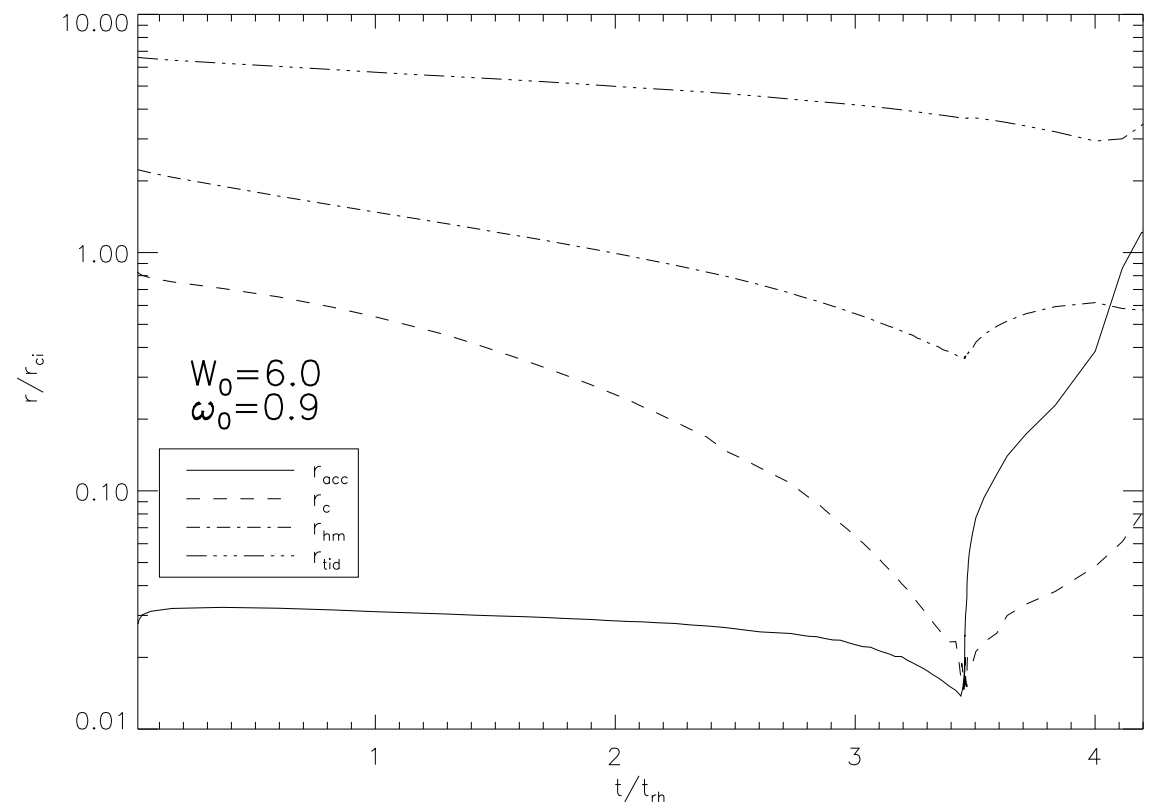

Figure 4.11: Evolution of cluster radii for the model B4 $\left(0.6,0.9,5 \cdot 10^{-5}\right)$. Accretion, core, half-mass and tidal radii, are given in units of initial $r_{\mathrm{c}}$ are indicated in the legend. 
to $\theta=54.74^{\circ}$. Here, the density $n(r, \theta)$ is calculated and the mass computed following:

$$
M(r)=\int_{0}^{r} 4 \pi n\left(r, 54.74^{\circ}\right) r^{2} d r
$$

Evolution of Lagrangian radii containing the indicated fractions of the initial mass is shown in Figs. (4.12) to (4.14), for models of different initial concentration and different rotation. Due to mass loss, the outer mass shells are rapidly depleted in all cases (faster for the high rotating models). Lagrangian radii give also a qualitative description of the interaction of a growing $\mathrm{BH}$ and the cluster mass shells. Initially, the $\mathrm{BH}$ mass growth is slow due to the low central density, and Lagrangian radii are determined by core contraction. As BH mass accretion rate accelerates, these are even faster depleted close to the collapse, what can be seen in the break of the Lagrangian lines at times close before the collapse time. This is a consequence of the higher core density and accretion rates at collapse time. Finally the collapse is halted and reversed while accretion rate slow down rapidly and the mass shells re-expand. In all cases the re-expansion is clearly seen, due to evaporation of stars from the cluster halo, which is faster for the higher rotating models. Note that the smallest Lagrangian radius contains only $0.01 \%$ of the cluster mass, in order to follow the evolution of mass shells closer to the BH.

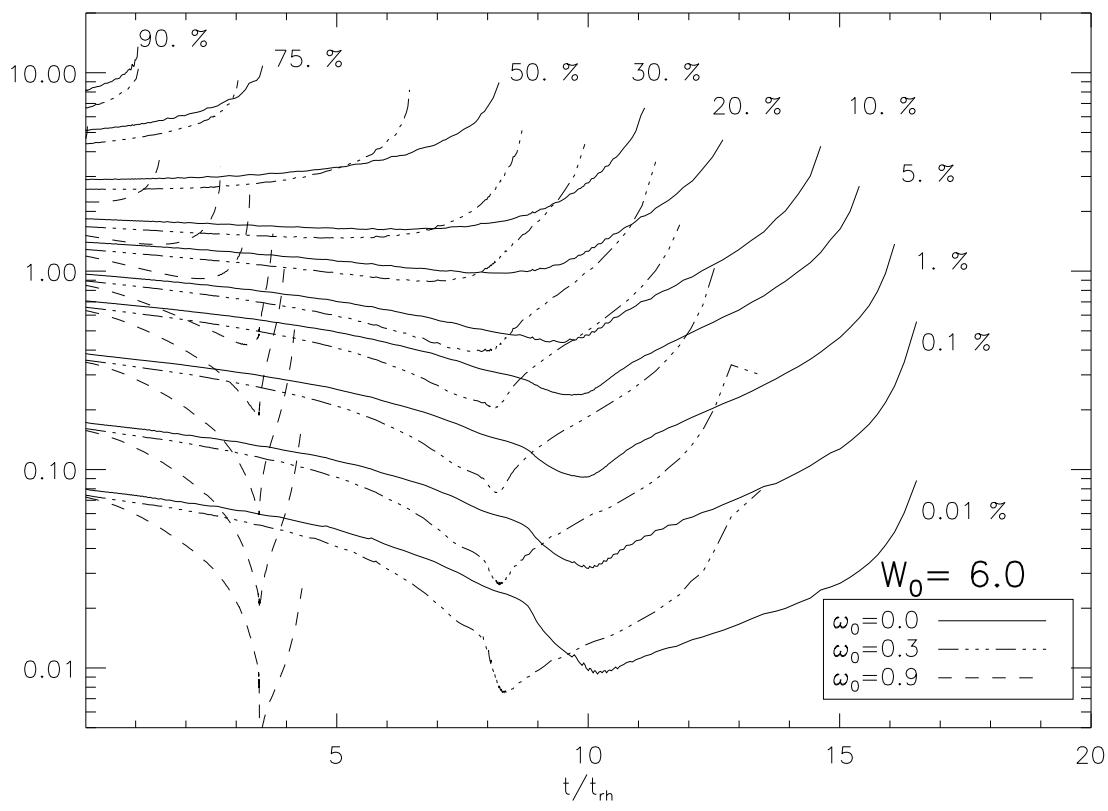

Figure 4.12: Evolution of mass shells (Lagrange radii $r_{55}$ ) for the models $W_{0}=6.0$ and initial rotation $\omega_{0}=0.0,0.3,0.9$. The percentage indicates the mass with respect to the total mass contained at the radii for mass columns in the direction of $\theta=54.74^{\circ}$. The time is given in units of initial half-mass relaxation time $\left(t_{\text {rhi }}\right)$.

A comparison with the $W_{0}=3$ models (Fig. 4.13) shows the influence of rotation on compact clusters. As mentioned, the evolution is faster than in the previous models, what can be seen in the reexpansion of the innermost mass shells, specially for the higher rotating models while the minimum radius of the mass shells slightly increases with higher initial 
rotation, as the maximum of density with higher rotation decreases in this models, due to an efficient transport of angular momentum outwards and faster mass loss. The concentrate $W_{0}=9$ models complete collapse even faster than the less concentrate models, and the gravogyro instability is more effective, leading to a rapid mass loss. Due to this effect, the outer mass shells are faster depleted (only the shell $75 \%$ can be seen in the plots). As mentioned, in all models, post-collapse is driven by outward heat flux from the cusp into the cluster core, since each disrupted star (orbit) gives energy to other stars (orbits) in relaxation time scales and the former fall to energies higher than $\left|E_{\text {crit }}\right|$, where accretion dominates.

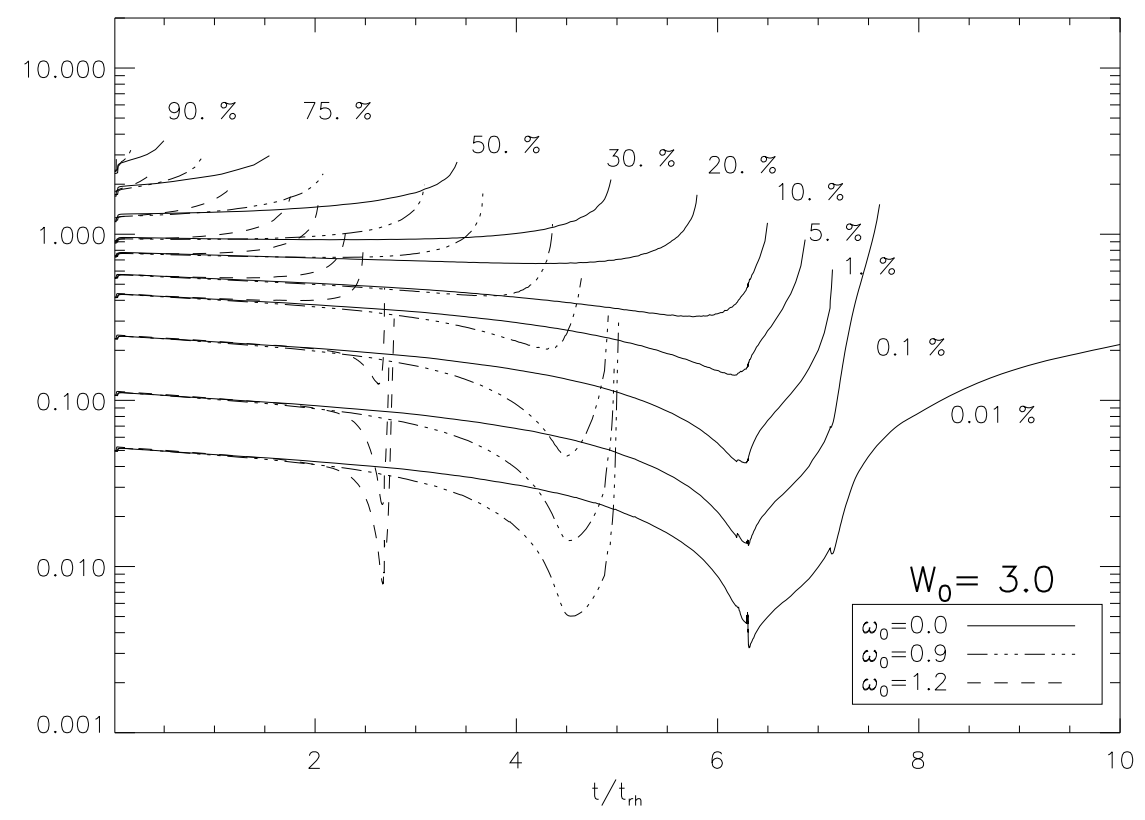

Figure 4.13: Evolution of mass shells (Lagrange radii $r_{55}$ ) for the models $W_{0}=3.0$ and initial rotation $\omega_{0}=0.0,0.9,1.2$. The percentage indicates the mass with respect to the total mass contained at the radii for mass columns in the direction of $\theta=54.74^{\circ}$. The time is given in units of initial half-mass relaxation time $\left(t_{\mathrm{rhi}}\right)$.

Another descriptor of collapse and core reexpansion in shown in Figure (4.15), where the density at the influence radius, $n_{\mathrm{a}} \equiv n\left(r_{\mathrm{a}}\right)$, is plotted as a function of the 1D total velocity dispersion, $\sigma_{\mathrm{a}} \equiv \sigma\left(r_{\mathrm{a}}\right)$. Evolution begins at the upper left part of the curves to the right. During the collapse phase, all curves follow growing density with time. Later, both $n_{\mathrm{a}}$ and $\sigma_{\mathrm{a}}$ fall during the post-collapse phase, as the cluster re-expands. The initial models start with a slightly different initial density at $r_{\mathrm{a}}$, higher for larger $\omega_{0}$, and follow in the $W_{0}=6$ case an evolution of collapse, characterized by a slow drop in $\sigma_{\mathrm{a}}$ for the higher rotating models. After the collapse, the velocity dispersion continues growing slightly, while the density drops. This is a good indicator of core heating, due to the central BH. Later, both density and dispersion decrease during the post-collapse phase in all models, following a similar trend, independent of rotation. The break of the curves shows the point of reverse of collapse, which depends on rotation, as seen previously, namely faster and at higher densities for the higher initially rotating models (one can consider the density as a transformed time axis). $W_{0}=3$ models exhibit a core cooling (lower $\sigma_{\mathrm{a}}$ ) as they evolve 


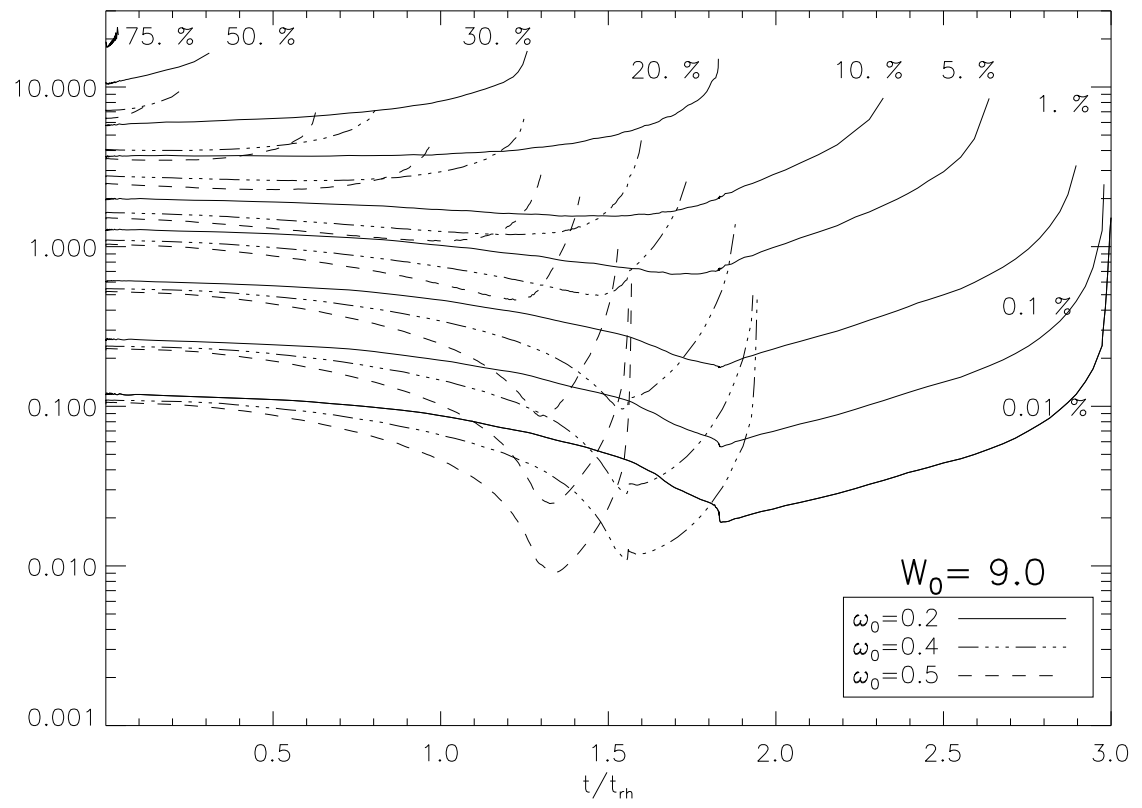

Figure 4.14: Evolution of mass shells (Lagrange radii $r_{55}$ ) for the models $W_{0}=9.0$ and initial rotation $\omega_{0}=0.2,0.4,0.5$. The percentage indicates the mass with respect to the total mass contained at the radii for mass columns in the direction of $\theta=54.74^{\circ}$. The time is given in units of initial half-mass relaxation time $\left(t_{\mathrm{rhi}}\right)$. 
apparently gravogyro driven to the collapse phase, what is clearly seen in the highest rotating model $\left(\omega_{0}=1.2\right)$. Effective angular momentum diffusion leads to a faster evolution of the cluster and a higher mass loss is a further consequence of this behavior. In all cases of the $W_{0}=9$ models, the core heats continuously towards collapse to higher densities and velocity dispersions, as these models start with concentration typical for collapsed systems. Both decrease during the post-collapse phase.

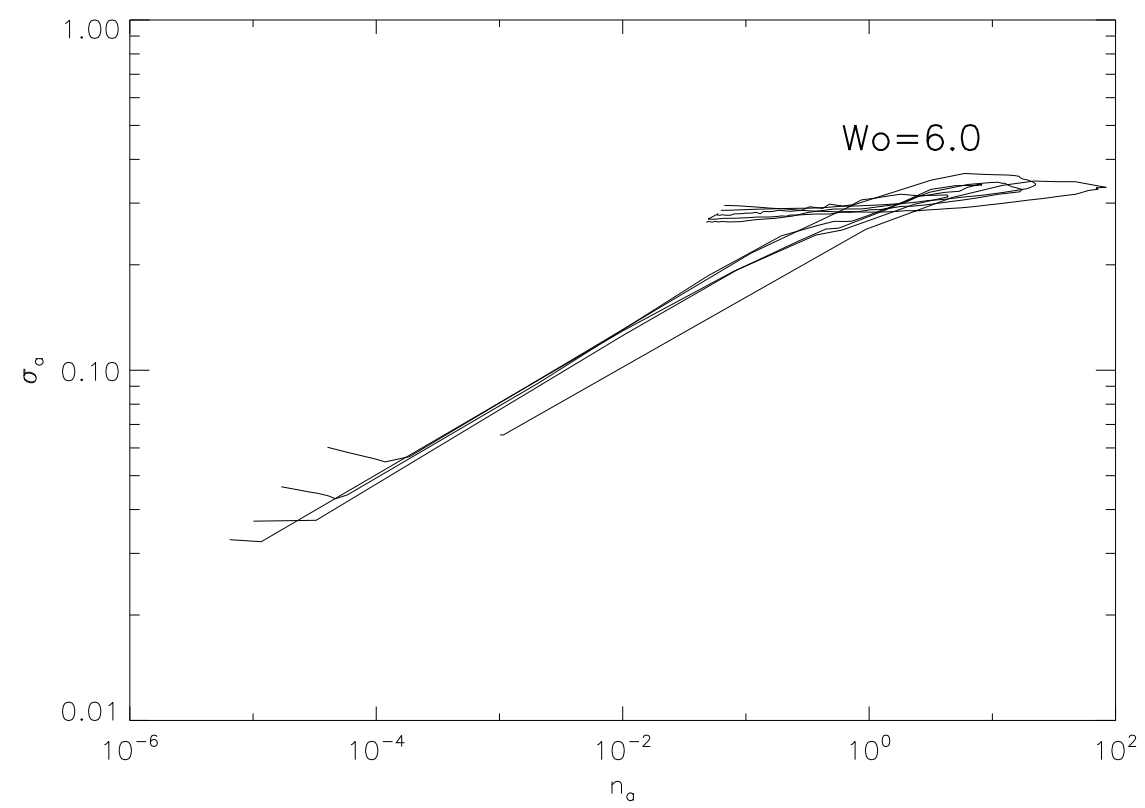

Figure 4.15: Evolution of 1D velocity dispersion with density, both evaluated at the $\mathrm{BH}$ influence radius for models of $W_{0}=6.0$ and initial rotation $\omega_{0}=0.0,0.3,0.6,0.9,1.2$

In this features, evolution up to the collapse phase and during the post-collapse is shown to depend on both, the concentration and rotation parameters. During the post-collapse phase, when the curves have changed their direction to the left due to the drop of density at $r_{\mathrm{a}}$ and a decrease in $\sigma\left(r_{\mathrm{a}}\right)$, evolution continues until cluster dissolution. In this later phase of core expansion, the influence radius moves faster from the center in time, as the $\mathrm{BH}$ grows, while, as will be discussed in the next section (4.3), rotation is carried away and mass is lost from the system.

As described in Sect. (3), the simulation begins with an embedded initial $\mathrm{BH}$ mass at $t=0.0$, centered in an homogeneous core. Throughout the calculation, the $\mathrm{BH}$ accretes stars in orbits of low $z$-component of angular momentum, what is calculated following the method described in Sect.(3.2.2). Thus $M_{\mathrm{BH}}$ grows driven by the accretion of energetic orbits generated through the potential cusp of the central mass and by angular momentum diffusion of stars outwards caused by relaxation effects. At the same time, the cluster loses mass through the galaxy tidal boundary.

As seen in Table (4.4), the cluster has lost, at collapse time, between 40 and $85 \%$ of its mass in the non-BH models and between 63 an $99 \%$ of the initial cluster mass in the $\mathrm{BH}$ 


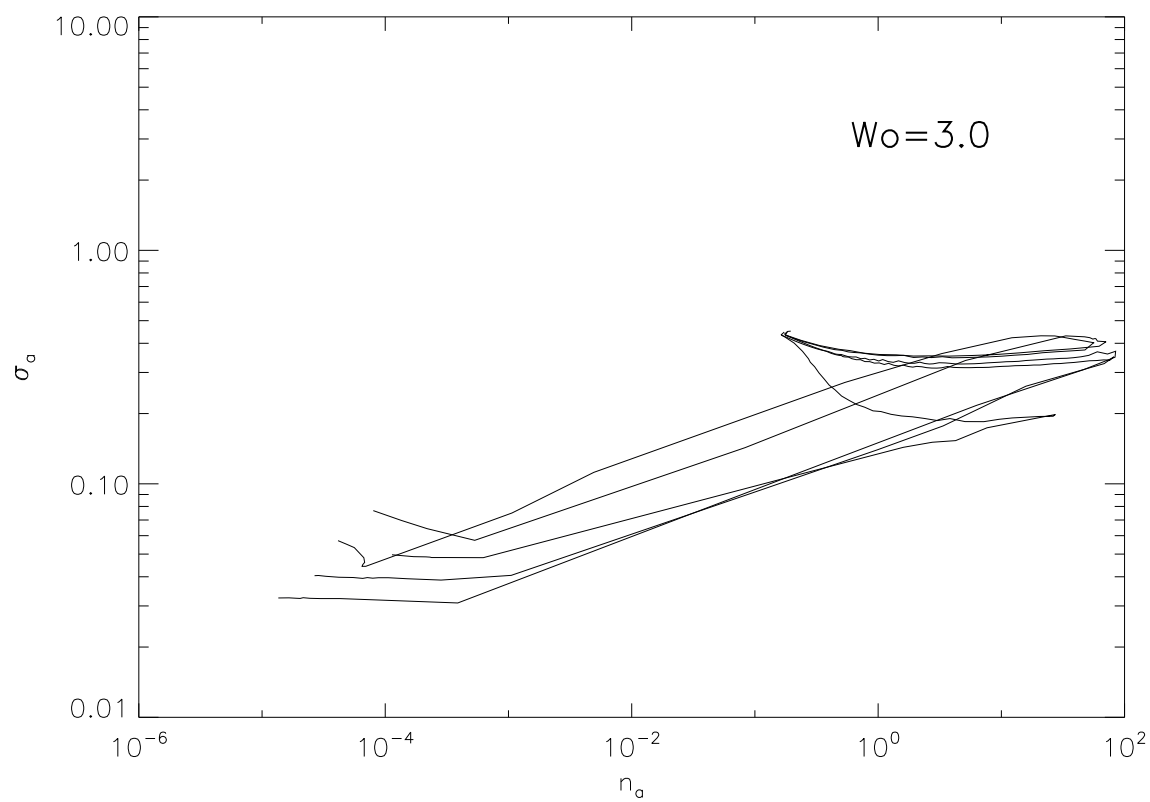

Figure 4.16: Evolution of 1D velocity dispersion with density, both evaluated at the $\mathrm{BH}$ influence radius for models of $W_{0}=3.0$ and initial rotation $\omega_{0}=0.0,0.3,0.6,0.9,1.2$

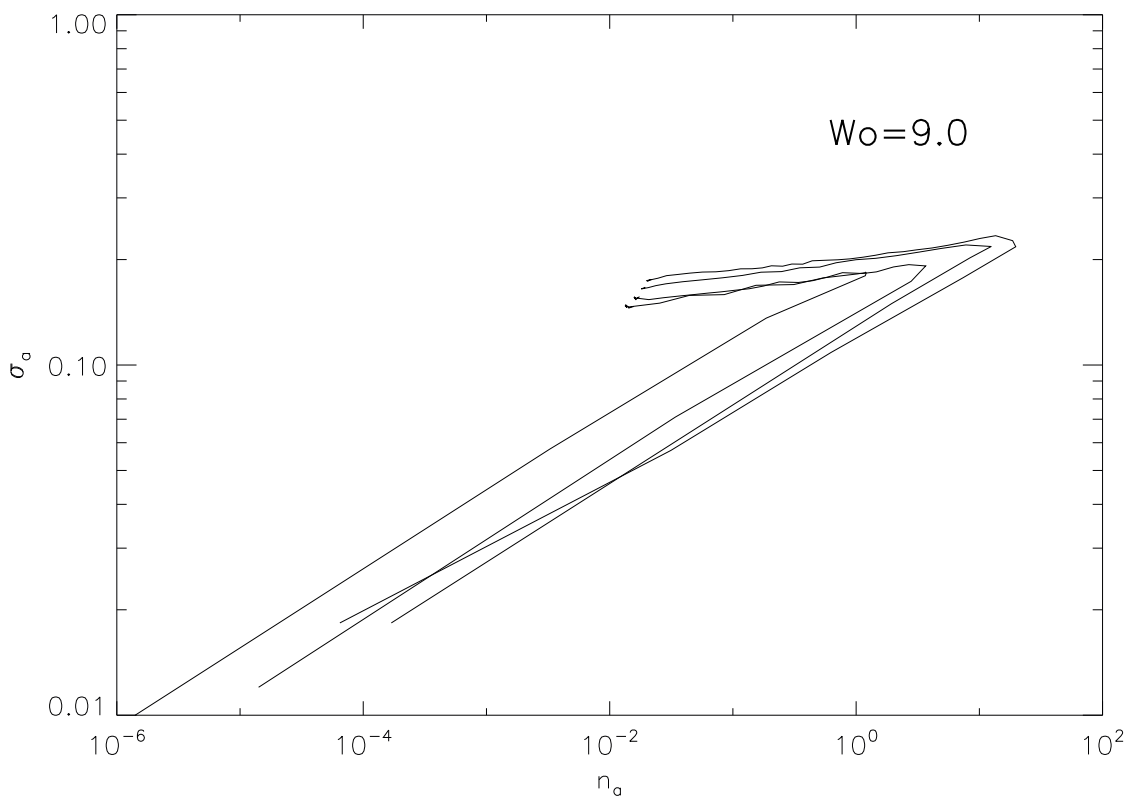

Figure 4.17: Evolution of 1D velocity dispersion with density, both evaluated at the BH influence radius for models of $W_{0}=9.0$ and initial rotation $\omega_{0}=0.2,0.3,0.4,0.5$ 
models. In both cases the mass loss is higher for higher rotation and less concentration of the initial models, as transport of angular momentum to the outer parts of the cluster leads to the disruption of halo stars mainly in circular orbits. The time at which the cluster loses half of its mass is shorter, the higher the initial rotation of the model. The acceleration of mass loss to the $50 \%$ of the initial mass is even higher than the acceleration of collapse. As seen in Fig. (4.18), BH accretion rate accelerates at times close to the collapse, when angular momentum diffusion is more effective and mass loss rate increases. This shows that, as well as rotation supports BH star accretion, the latter leads to a more efficient transport of angular momentum. Thus, evolution is driven by the interplay between gravogyro instabilities and BH star accretion, both driven by relaxation effects. This will also involve a much faster dissolution of the cluster through galactic tidal effects.

Fig. (4.18) shows for the model B3 $\left(6.0,0.6,5 \cdot 10^{-6}\right)$ the evolution of cluster $\left(M_{\mathrm{cl}}\right)$ and $\mathrm{BH}$ mass $\left(M_{\mathrm{BH}}\right)$ together with the accretion rate (in code mass and relaxation time units). It can be seen how the BH-mass reaches a constant fraction of the initial cluster mass $(\sim 1 \%)$ at collapse time and remains nearly constant in the post-collapse phase $\left(t / t_{\text {rhi }} \gtrsim 6\right)$, while the star accretion rate $(d M / d t)$ is maximal at collapse time due to the higher density of orbits in the core and decrease very fast afterwards. The cluster mass loss due to tidal effects of the parent galaxy is very strong during the re-expansion of the core as one can see in the evolution of $M_{\mathrm{cl}}$. The cluster mass reaches very rapid values at least one order of magnitude smaller than the mass of the $\mathrm{BH}$. The acceleration of mass loss is similar as the observed by Kim et al. (2002) in the post-collapse models driven by binary heating, although the effect in the present BH-models is more pronounced, with the consequence of a faster evolution of the cluster towards sphericity (see also Fig. 4.37) and final dissolution.

As can be seen in Figure (4.19) the evolution of $\mathrm{BH}$ and cluster mass for different rotation parameters $\left(\omega_{0}=0.9,0.6,0.3,0.0\right.$ for curves from left to right) is comparable and qualitative similar among them. The stronger angular momentum diffusion to the outer parts of the core and as a consequence the enhanced mass loss at post-collapse time in high rotating models together with the strong accretion rate, are caused by the predominance of high energetic radial orbits in the system, which can lose easily energy through collisions with other stars and fall to the $\mathrm{BH}$ potential cusp, leading to a faster final evolution of the cluster, while a more massive $\mathrm{BH}$ forms due to effective accretion, thus in direct relation to the initial cluster rotation. The final BH-mass approaches roughly $1 \%$ of $M_{\mathrm{cl}}$ always at earlier times (from a maximum of $9 \cdot 10^{-3}$ for the non-rotating case to $\sim 2 \cdot 10^{-2}$ for the highest rotating model, see Table 4.5). The BH-mass limit for a total number of stars used in this simulations of $5 \cdot 10^{6} M_{\odot}$, varies between $1.9 \cdot 10^{3} M_{\odot}$ and $9.5 \cdot 10^{4} M_{\odot}$, which is a good approximation of the mass of IMBH estimated by theoretical studies and observations (see Sects. 2.3 and 1.4).

Table (4.5) shows the final $\mathrm{BH}$ mass limits of each model and the respective maximal accretion rates at the time $M_{\mathrm{BH}}$ stalls and the accretion rate begins to slow down. As can be seen in Fig. (4.19), the cluster mass has dropped already to very small values as the BH mass growth rate starts falling as the cluster approaches its final dissolution. Table (4.5) gives also the final $\mathrm{BH}$ mass in units of solar mass and the maximal accretion rates in solar mass per year. To this purpose, the cluster mass was set to $M_{\mathrm{cl}_{\mathrm{i}}}=5 \cdot 10^{6} M_{\odot}$ and the core radius to $r_{\mathrm{c}_{\mathrm{i}}}=10 \mathrm{pc}$. The half-mass relaxation time was calculated following Eq. (1.4), 


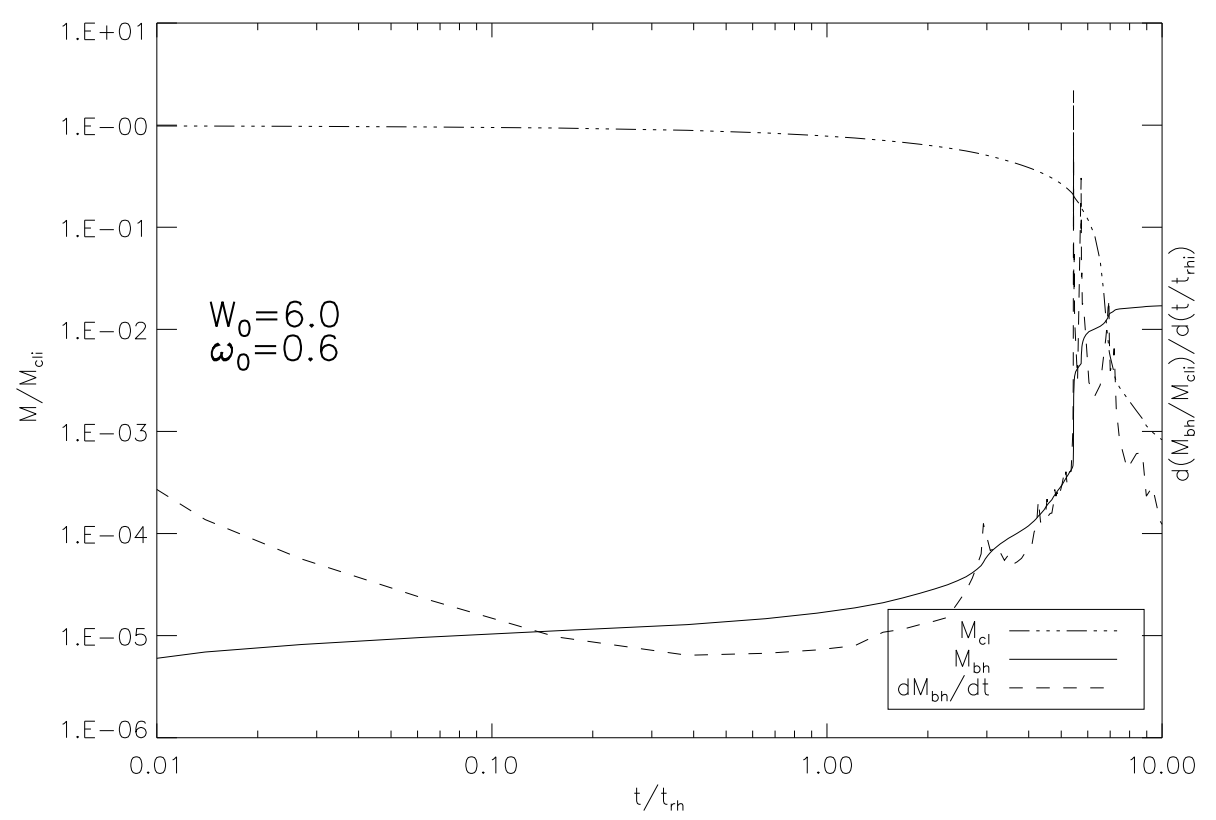

Figure 4.18: Evolution of BH and cluster mass for the model B3 $\left(6.0,0.6,5 \cdot 10^{-6}\right)$. The dot-dashed line shows the mass of the cluster and the solid line the BH mass. Mass accretion rate is showed as a dashed line in units of $d\left(M_{\mathrm{BH}} / M_{\mathrm{cl}_{\mathrm{i}}}\right) / d\left(t / t_{\mathrm{rh}_{\mathrm{i}}}\right)$. The time is given in units of initial half-mass relaxation time $\left(t_{\text {rhi }}\right)$.

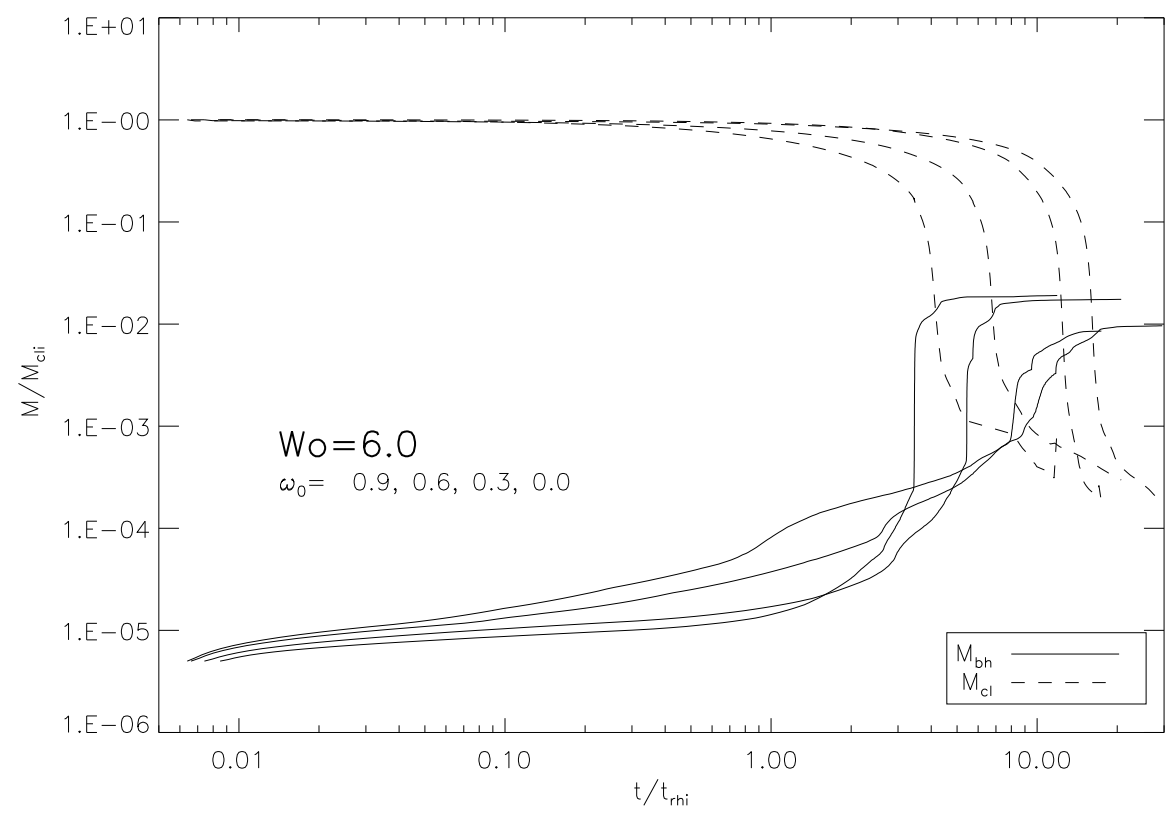

Figure 4.19: Time evolution of cluster mass (green line) and BH-mass (black line). The red line shows the evolution of mass accretion rate $d M / d t$. Initial $M_{\mathrm{bh}}=5 \cdot 10^{-6} M_{\mathrm{cl}_{\mathrm{i}}}\left(25 M_{\odot}\right.$ for this model $)$ and final $M_{b h} \simeq 0.01 M_{\mathrm{cl}_{\mathrm{i}}}\left(5000 M_{\odot}\right)$ 


\begin{tabular}{|c|c|c|c|c|}
\hline Model name & $M_{\mathrm{BH}}^{\text {stall }}$ & $M_{\mathrm{BH}}^{\text {stall }}\left(M_{\odot}\right)$ & $d M / d t_{\max }$ & $d M / d t_{\max }\left(M_{\odot} / \mathrm{yr}\right)$ \\
\hline \hline $\mathrm{A} 1$ & $4.3 \cdot 10^{-4}$ & $2.1 \cdot 10^{4}$ & 0.038 & $1.77 \cdot 10^{-3}$ \\
\hline $\mathrm{A} 2$ & $5.5 \cdot 10^{-4}$ & $2.7 \cdot 10^{4}$ & 0.040 & $1.32 \cdot 10^{-3}$ \\
\hline $\mathrm{A} 3$ & $6.0 \cdot 10^{-4}$ & $3.0 \cdot 10^{3}$ & 0.043 & $1.21 \cdot 10^{-3}$ \\
\hline $\mathrm{A} 4$ & $8.9 \cdot 10^{-4}$ & $4.4 \cdot 10^{3}$ & 0.010 & $2.83 \cdot 10^{-4}$ \\
\hline $\mathrm{A} 5$ & $1.0 \cdot 10^{-3}$ & $5.0 \cdot 10^{3}$ & 0.104 & $3.24 \cdot 10^{-3}$ \\
\hline \hline $\mathrm{B} 1$ & $9.6 \cdot 10^{-3}$ & $4.8 \cdot 10^{4}$ & 0.030 & $3.40 \cdot 10^{-4}$ \\
\hline $\mathrm{B} 2$ & $8.5 \cdot 10^{-3}$ & $4.2 \cdot 10^{4}$ & 0.035 & $4.74 \cdot 10^{-4}$ \\
\hline $\mathrm{B} 3$ & $1.7 \cdot 10^{-2}$ & $8.5 \cdot 10^{4}$ & 0.102 & $1.39 \cdot 10^{-3}$ \\
\hline $\mathrm{B} 4$ & $1.9 \cdot 10^{-2}$ & $9.5 \cdot 10^{4}$ & 0.262 & $3.78 \cdot 10^{-3}$ \\
\hline $\mathrm{B} 5$ & $9.1 \cdot 10^{-3}$ & $4.5 \cdot 10^{4}$ & 0.892 & $1.30 \cdot 10^{-2}$ \\
\hline \hline $\mathrm{C} 1$ & $1.0 \cdot 10^{-3}$ & $5.0 \cdot 10^{3}$ & 0.371 & $5.38 \cdot 10^{-4}$ \\
\hline $\mathrm{C} 2$ & $4.6 \cdot 10^{-4}$ & $2.3 \cdot 10^{3}$ & 0.009 & $1.87 \cdot 10^{-5}$ \\
\hline $\mathrm{C} 3$ & $2.9 \cdot 10^{-4}$ & $1.5 \cdot 10^{3}$ & 0.002 & $4.29 \cdot 10^{-6}$ \\
\hline $\mathrm{C} 4$ & $3.8 \cdot 10^{-4}$ & $1.9 \cdot 10^{3}$ & 0.001 & $4.76 \cdot 10^{-6}$ \\
\hline
\end{tabular}

Table 4.5: Description of mass parameters. Column 1: Model name; Column 2: Final $M_{\mathrm{bh}}$ as it reaches an asymptotic mass; Column $3: M_{\mathrm{bh}}$ in $M_{\odot}$-units; Column 4: maximal accretion rate $d M / d t$; Column4: $d M / d t$ in $\left(M_{\odot} / \mathrm{yr}\right)$-units. 
after scaling the half-mass radius to the initial core radius using Table (4.2).

The general behavior exhibits an increasing final $\mathrm{BH}$ mass as well as higher mass growth rates corresponding to higher initial rotation. Nevertheless, the high concentrated models show the inverse phenomena, a decreasing final $M_{\mathrm{BH}}$ and maxima of $d M_{\mathrm{BH}} / d t$ with higher rotation. The reason could be the accelerated evolution through the dominant gravogyro effect and consequent higher tidal mass loss with higher rotation and inefficient $\mathrm{BH}$ accretion due to lower initial central densities in this models. Note that the time interval between the collapse time $t_{\mathrm{cc}}$ of Table (4.4) and the time when $M_{\mathrm{BH}}$ stalls is always shorter the higher the rotation of the model. Marchant \& Shapiro (1980) follow the evolution of a star cluster containing a central $\mathrm{BH}$ included in the simulations at the collapse time. The BH mass stalls after approximately 2 relaxation time units to a final mass of $\sim 4000 M_{\odot}$. In rotating models, a stalling of $M_{\mathrm{BH}}$ is observed always at shorter times when the rotation parameter $\omega_{0}$ is higher. It varies from $10 t_{\mathrm{rh}}$ (for non-rotating models) to $0.1 t_{\mathrm{rh}}$ (for high rotating models). The maximum of $M_{\mathrm{BH}}$ depends also on initial concentration, but mainly on rotation (Table 4.5). Despite of the difference in the methods used (Monte Carlo and Fokker Planck) and system structure (spherically symmetric and axisymmetric), the rapid evolution after collapse and asymptotic behavior of $M_{\mathrm{BH}}$ in time is a general common feature.

\subsection{Rotation and angular momentum}

In models without $\mathrm{BH}$, the total collapse time is shortened by the gravo-gyro effect (Hachisu, $1979,1982)$, by which large amounts of initial rotation drive the system into a phase of strong mass loss while it contracts (the core rotates faster although angular momentum is transported outwards). At the same time, the core is heating, while the source of the so called 'gravo-gyro' catastrophe is consumed and the growth in central rotation levels off after 2 $3 t_{\mathrm{rh}}$ towards core collapse (Einsel \& Spurzem 1999). Simulations into post-collapse phase, driven by three-body binary heating shown by Kim et al. (2002) exhibit a faster evolution of the cluster up to its final dissolution. Cluster dissolution is faster for clusters with higher initial rotation, although the overall shape of cluster (i.e. flattening) changes little after core collapse, independent of initial rotation. Ardi et al. (2005) use N-Body simulations of initially rotating clusters and confirm the predicted faster evolution driven by outward transport of angular momentum due to gravogyro instability up to collapse, followed by a slow expansion. In this section, the effect of cluster initial rotation on redistribution of orbits under the influence of the central $\mathrm{BH}$ and star accretion, during collapse as well as in the post-collapse phase, is studied.

$\mathrm{BH}$ models experience in a similar way, the onset of gravogyro instabilities, as angular momentum diffuses outwards, leading to an increase of central rotation. As BH accretion of stars on orbits of low $z$-component of angular momentum sets off, an ordered motion of high-angular momentum bounded orbits around the central $\mathrm{BH}$, is built, supporting central rotation. At the same time, stars in the core are heated via the consumption of stars in bound, high energetic orbits in the cusp. The outward flux of energy is achieved by small angle, two-body encounters in the cusp, by which some stars lose energy and move closer to the $\mathrm{BH}$ being eventually consumed, while the neighboring stars with which they interact 
gain energy and move outward from the cusp into the ambient core. On the other side, gravogyro effects carry out angular momentum from the core, increasing in this way the population of stars in low- $J_{\mathrm{z}}$ orbits, and thus the $\mathrm{BH}$ mass accretion rate. At the same time, mainly unbounded circular tidal orbits in the halo are disrupted at the tidal boundary. As discussed in the previous section, mass loss is enhanced through this effects and $\mathrm{BH}$ accretion is supported by angular momentum diffusion.

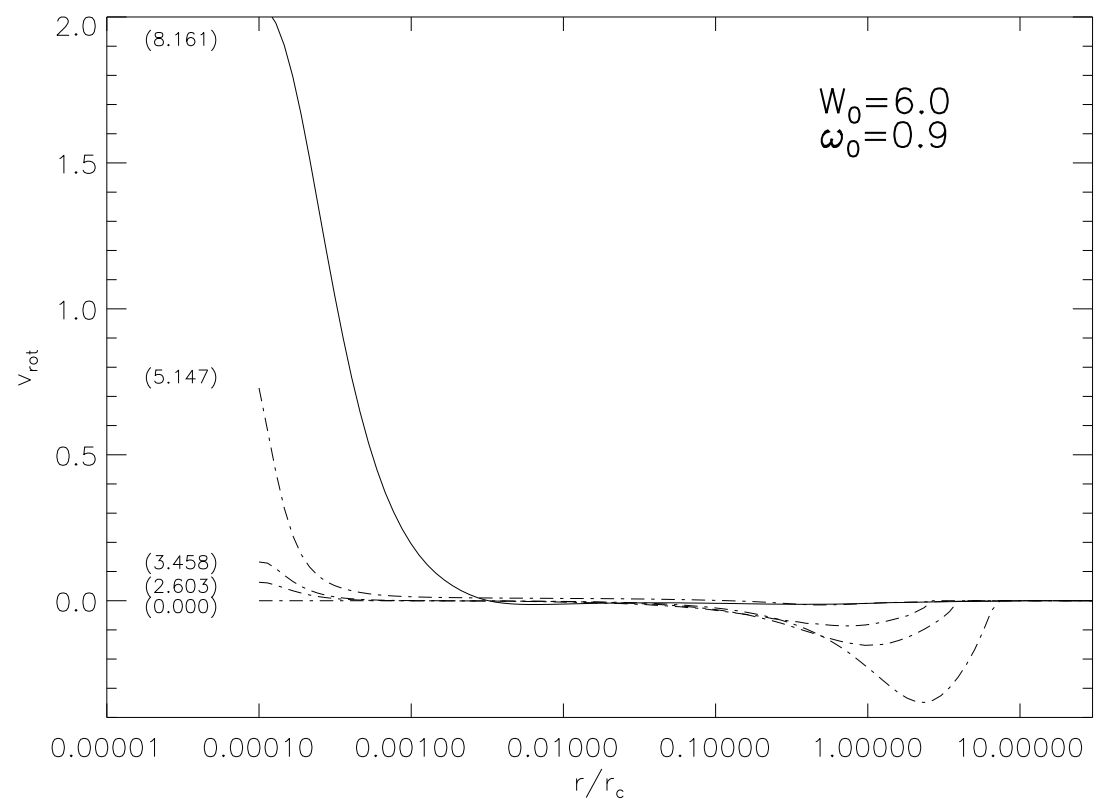

Figure 4.20: Evolution of rotational velocity profile for the model B4 $\left(6.0,0.9,5 \cdot 10^{-6}\right)$. Note the formation of a maxima of rotation in the central region. Time is given parenthesis, in units of initial half-mass relaxation time $\left(t_{\mathrm{rhi}}\right)$.

Thus, as the $\mathrm{BH}$ grows and rotation increases in its zone of influence $\left(r<r_{\mathrm{a}}\right)$ angular momentum continues being transported out of the core. Fig. (4.20) shows the evolution of rotational profile for a model B4 (time is given in units of $t_{\mathrm{rh}}$ ) and Fig. (4.21) shows snapshots of the evolution of its 2-dimensional distribution in the meridional plane, at representative times, where the lighter areas represent contours of higher rotation. As can be seen, central rotational velocity grows slowly in the initial evolutionary states, and develops during the evolution up to collapse time, when the maxima of rotation $\left(V_{\mathrm{rot}}^{\max }\right)$ at the center dominates over the original maxima at around the half-mass radius, which has been present in the initial models and drops slowly in time (bottom right figure in Fig. 4.21) and solid line in Fig. (4.20). In the outer parts, rotation is continuously depleted, due to angular momentum loss. Later, when the system reaches the end of evolution, i.e. close to cluster dissolution, it has lost nearly all its rotational energy.

As can be seen in Fig. (4.20), central rotational velocity grows in opposite direction with respect to the halo. Initially, the cluster exhibits solid body negative rotation in all regions, with a maximum at the half-mass radius and the $z$-axis as rotation axis. Positive central rotation is built in the center under the influence of the $\mathrm{BH}$ potential cusp, in a similar 
a) $t / t_{r h i}=0.00$

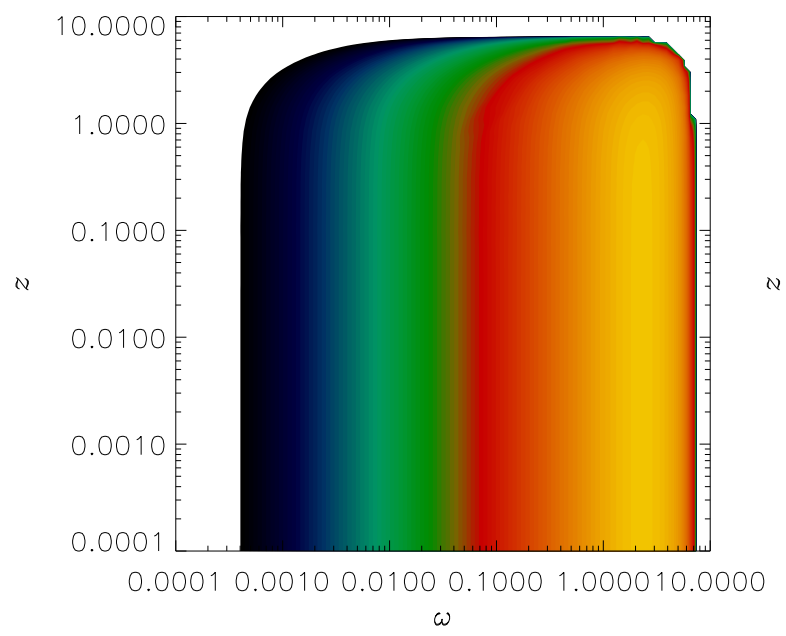

c) $t / t_{r h i}=3.46$

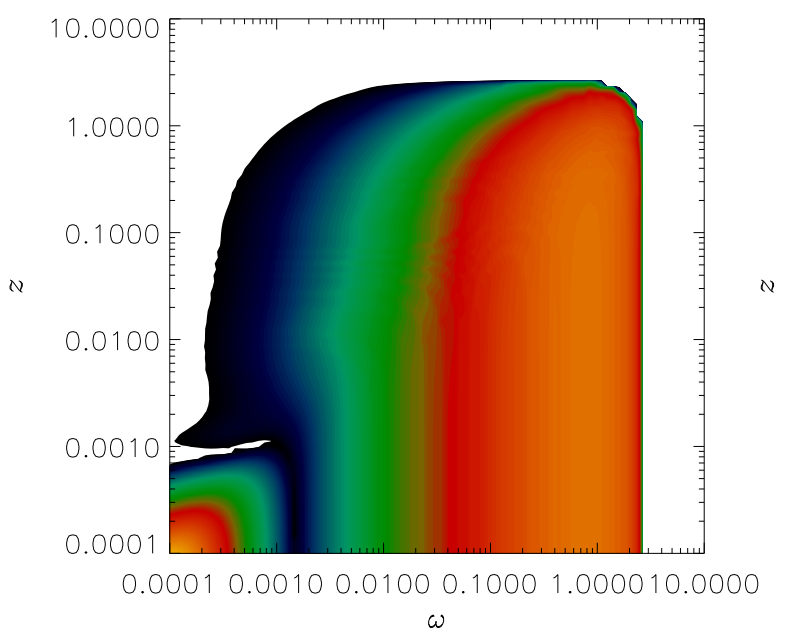

b) $t / t_{\text {rhi }}=2.60$

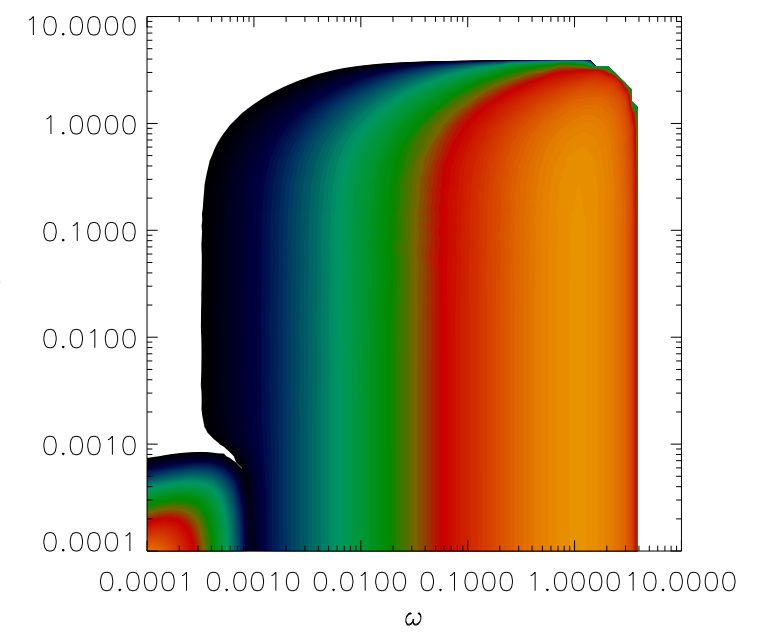

d) $t / t_{\text {rhi }}=5.43$

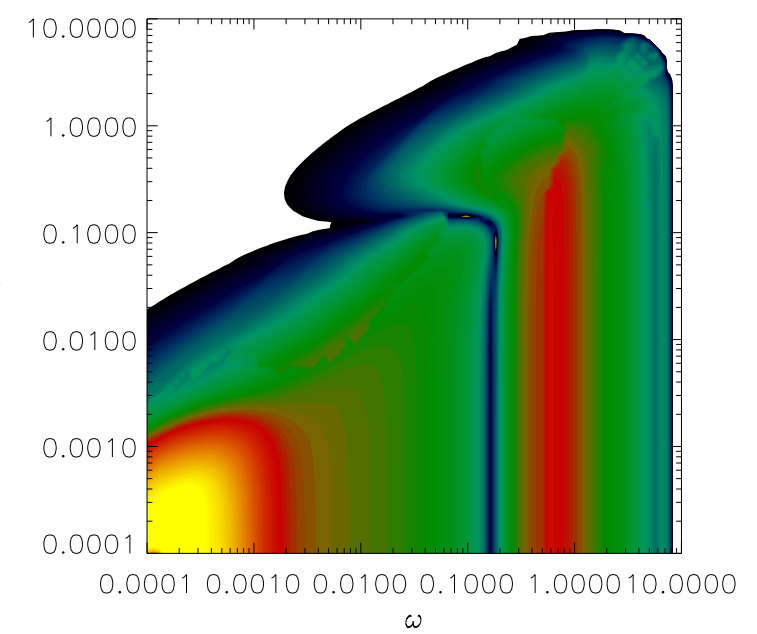

Figure 4.21: 2D rotational velocity distribution in the meridional plane for a model B4 $(6.0,0.9,5$. $\left.10^{-6}\right)$. Note the formation of a maxima of rotation in the central region. Time is given in units of initial half-mass relaxation time $\left(t_{\text {rhi }}\right)$. 
way as pro-grade (negative) stellar orbits in the halo, are initially preferably lost through the outer boundary due to the galactic tidal field, depleting initial negative rotation slowly, while positive angular momentum (i.e. rotation, dot-dashed lines in Fig. 4.20) dominates. On the other side, close to the $\mathrm{BH}$, where angular momentum is initially small (for tightly bounded stars), orbits are preferably ordered in positive direction, apparently, due to higher diffusion (and depletion) of negative angular momentum orbits into the loss-cone. As halo stars interact with core stars more than with other halo stars, tidal galaxy effects influence also the redistribution of orbits in the core. Note that the diffusion of energy and angular momentum is a result of relaxation effects, due to small angle interactions of orbits, and no real collisions of stars or the existence of interstellar gas or stellar evolution is considered. See also Sect. (4.4) for a further discussion.

During cluster evolution, high energy, low- $J_{\mathrm{z}}$ (radial) orbits with large apocenters are created through energy diffusion, and populate the halo regions, leading to radial (positive) anisotropy at large radii. This orbits can be easily lost from the cluster due to diffusion effects towards core collapse, which efficiency determines the degree of anisotropy in the outer regions (Fig. 4.8). At the same time, in the inner regions, mainly radial orbits are accreted inside the $\mathrm{BH}$ radius of influence, leading, as discussed in the last section, to a dominance of circular orbits (negative central anisotropy). Moreover, relaxation effects in the halo lead to interaction of halo stars mainly with stars in the core rather than with other halo stars, owing to the low halo density. Transfer of energy and angular momentum to these orbits, reduces their eccentricities or leads to their loss at the tidal boundary or accretion by the $\mathrm{BH}$ (if their energies are high and their angular momentum small enough, inside the loss-cone). As the system loses its angular momentum due to gravogyro instabilities, more stars are transported from the core to the outer regions, depleting the population of core orbits, and consequently, core rotation.

As another indicator of system rotation, the relation

$$
\Omega_{a} \propto n_{a}{ }^{\delta}
$$

introduced by Goodman (1983) is used here, evaluated at the $\mathrm{BH}$ influence radius $\left(r_{\mathrm{a}}\right)$. Figs. (4.22) to (4.24) show the evolution of density $\left(n_{\mathrm{a}}\right)$ with angular velocity $\left(\Omega_{\mathrm{a}}\right)$ for all models, using different initial rotations. In all cases, gravogyro effects are responsible for an increase in $\Omega_{\mathrm{a}}$ at early evolutionary times (Eq. 2.1). For the models $W_{0}=6.0$ and $W_{0}=9.0$, the growth is maintained during the collapse phase, what is not the case of the model $W_{0}=3.0$. In the model $W_{0}=6.0$ rotational velocity decreases slightly close to collapse, after an initial moderately increasing at early times, and shows also a short increasing after the collapse, when the density already starts to fall due to the expansion of the core, falling even faster afterwards. Note a similar evolution of the velocity dispersion in this models shown in Fig. (4.15). Nevertheless, as central rotation grows towards collapse, the systems uses part of its total energy to support rotation, leading to a slower decrease in velocity dispersion. The growth of rotation, and dispersion, shortly after collapse, is not observed in the other models.

The models $W_{0}=0.9$ show a faster increase of $\Omega_{\mathrm{a}}$ at earlier times and a moderate growth towards collapse, with respect to the initial values of angular velocity. Nevertheless, the absolute values of angular velocity in this models lay below the ones in less concentrated 
models. The models $W_{0}=0.3$ show in all cases a short initial increase followed by a very rapid decrease in $\Omega_{\mathrm{a}}$ by increasing density, still before the collapse is reversed. This effect is stronger, the rapid the rotation of the initial models, due to a rapid depletion of angular momentum through relaxation in the low concentrated initial configurations, at always shorter collapse times. In all cases, the curve turns later down during post-collapse evolution to lower $\Omega_{\mathrm{a}}$ and $n_{\mathrm{a}}$.

As a consequence of angular momentum diffusion, rotation continues being depleted in the system, even faster after collapse, and the growth of rotation towards the center is determined by this effect. From Figs. (4.22) to (4.24) depletion of central rotation seems to happen earlier for $W_{0}=3.0$ models, while rotation in the most concentrated models continues increasing during collapse. From this one comes to the conclusion, that the amount of rotation present in the core at later times, i.e. at collapse and afterwards, depends on the efficiency of angular momentum diffusion due to relaxation and BH star accretion, specially before most of angular momentum is driven away. Central rotation supports ordered orbital motion around the $\mathrm{BH}$ and increases the star accretion efficiency, leading to a higher final $M_{\mathrm{BH}}$, before it stalls and the accretion rate drops, as observed in the higher initially rotating models of Fig. (4.19). Like in non-BH models, angular momentum loss accelerates in the post-collapse time (Kim et al. 2002), leading to final cluster dissolution.

Furthermore, the preferred $\varpi$-direction (in the equatorial plane) of density growth mentioned in the last section (Fig. 4.4d) is also observed in the $2 \mathrm{D}$ profile of rotational velocity (Fig. 4.21d), what confirms the influence of rotation in the redistribution of stars in the cluster, in the sense that ordered motion of bounded stars around the $\mathrm{BH}$ predominates in the equatorial plane, more than in regions close to the rotation axis.

Figures (4.25) to (4.27) show for all models the time evolution of energy fraction of rotation with respect to the total energy of the system. The initial behavior is similar in all cases, and $T_{\text {rot }}$ drops slowly. As angular momentum is driven away from the core due to two-body relaxation effects, rotational energy is lost faster for the high rotating models, while the cluster collapses. By the time of core collapse (shown by arrows in Fig. 4.25), the rotational energy becomes rapidly only a small fraction of the total energy (see Table 4.6). In the post-collapse phase, rotation decreases even faster than before, being continuously depleted, while high energy orbits dominate, generated by the central energy source of the growing $\mathrm{BH}$ central potential, which influence radius keep growing with its mass, while the core expands. Thus, BH-models lose angular momentum faster than non-BH models, leading to an earlier collapse, as a consequence of gravogyro and gravothermal instabilities, and faster cluster dissolution in the post-collapse phase, due to a higher tidal mass loss.

Table (4.6) shows the collapse parameters of rotation for all models. The general decrease in the collapse time with rotation was already discussed under the influence of BH star accretion (Table 4.4). The rotational energy of the system decreases faster, the higher the initial rotation. Note that the initial rotational energy in low-concentration $W_{0}=3.0$ models is smaller than in models with higher concentration $\left(W_{0}=6.0,9.0\right)$, and thus, the cluster becomes rounder faster in time in the former models due to the efficiency of angular momentum diffusion. Moreover, the faster loss of angular momentum in the highest rotating model (A5) causes the depletion of rotational energy much earlier than the slower rotating models, and it shows the smallest rotational energy and ellipticity at collapse time (see also 


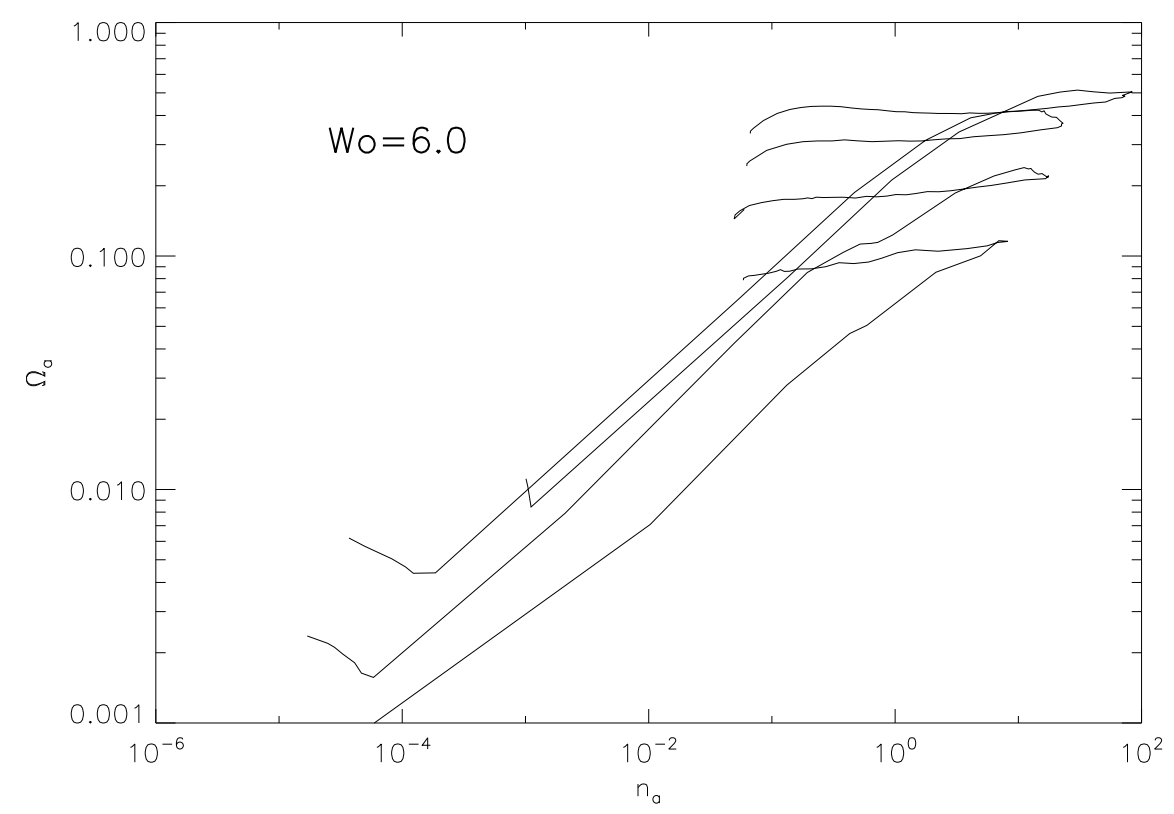

Figure 4.22: Evolution of angular velocity with density, both evaluated at the BH influence radius for models of $W_{0}=6.0$ and initial rotation $\omega_{0}=0.3,0.6,0.9,1.2$

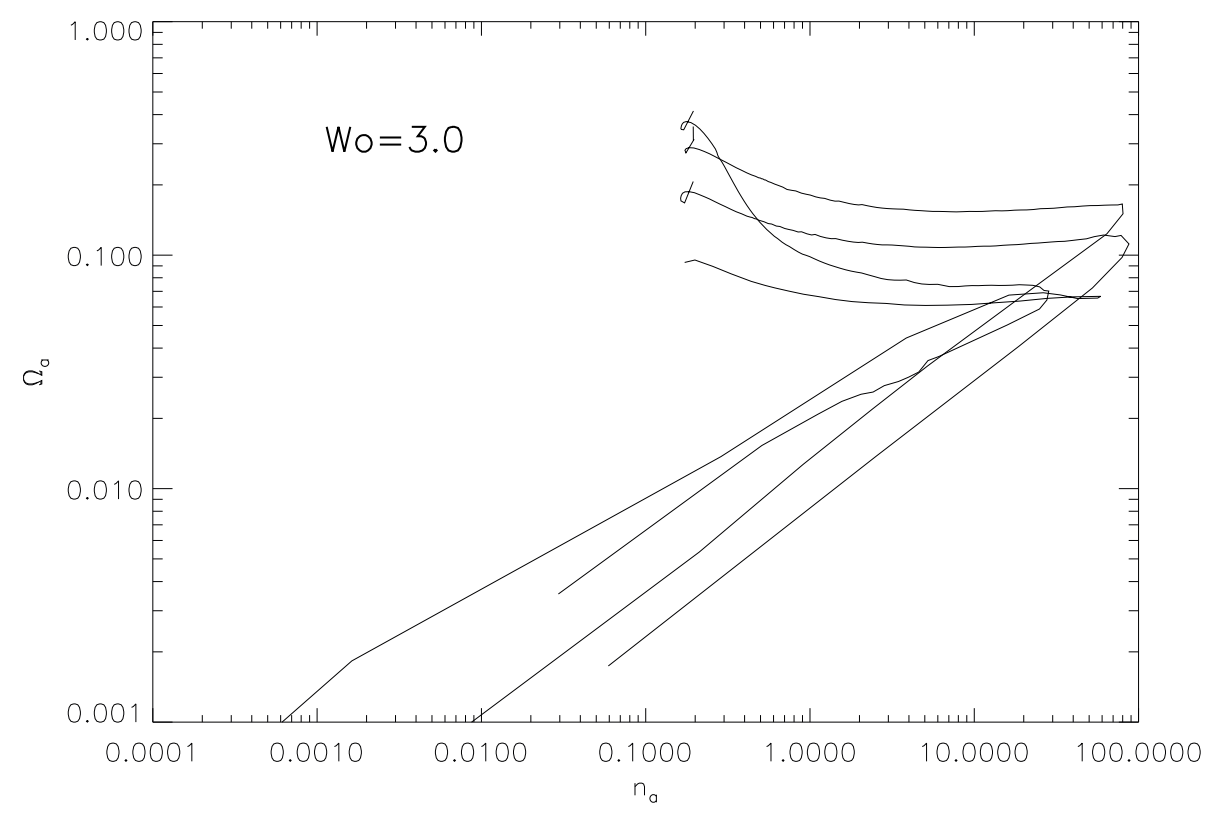

Figure 4.23: Evolution of angular velocity with density, both evaluated at the BH influence radius for models of $W_{0}=3.0$ and initial rotation $\omega_{0}=0.3,0.6,0.9,1.2$ 


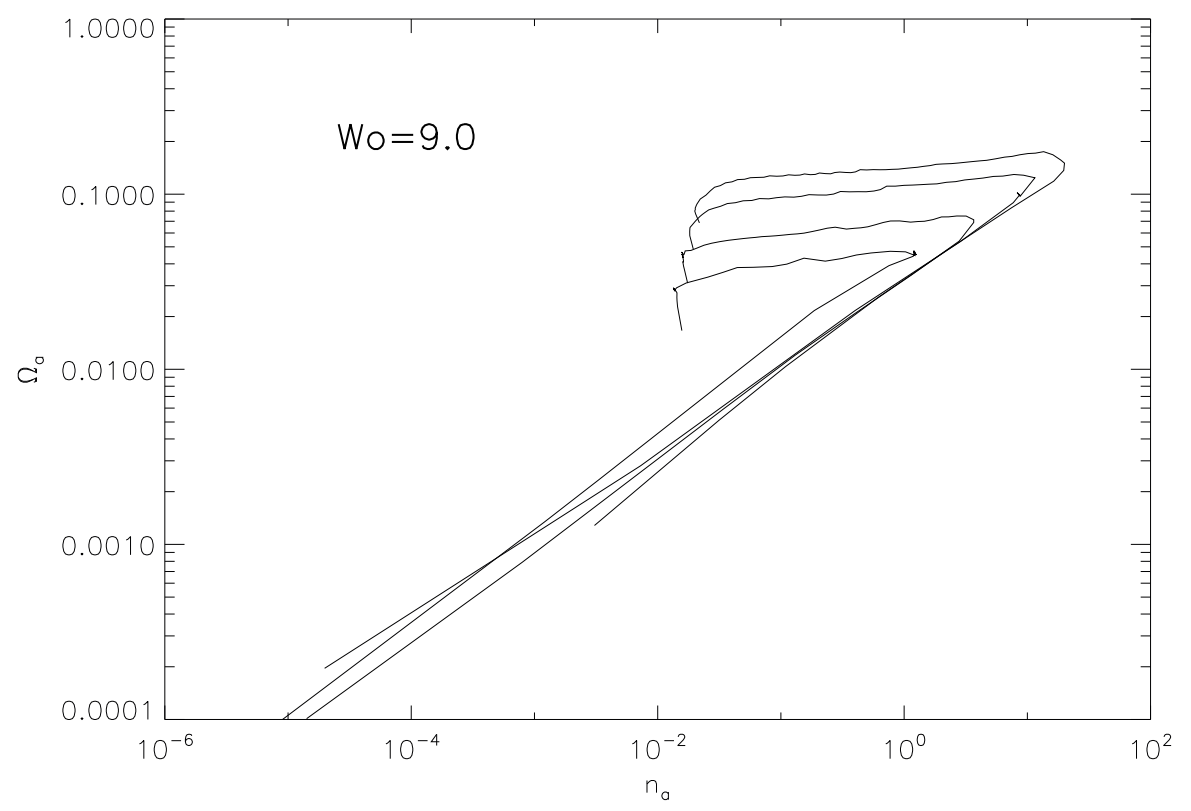

Figure 4.24: Evolution of angular velocity with density, both evaluated at the BH influence radius for models of $W_{0}=9.0$ and initial rotation $\omega_{0}=0.2,0.3,0.4,0.5$

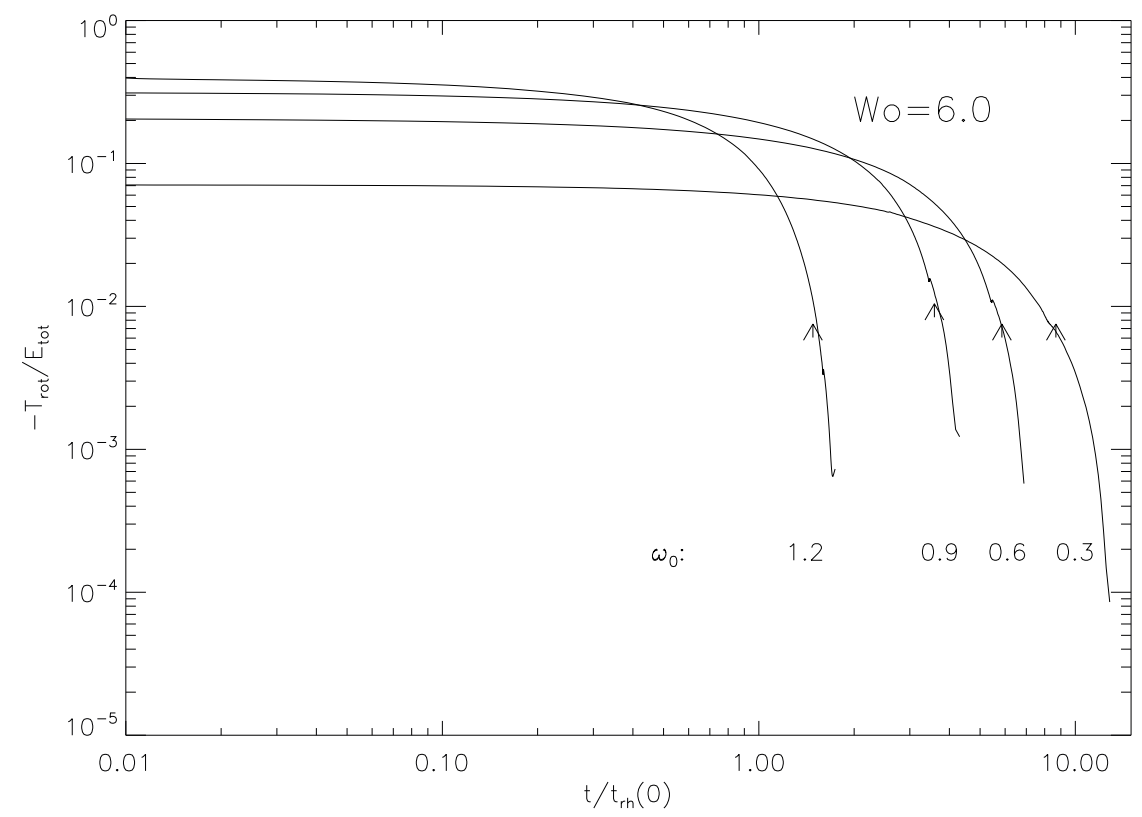

Figure 4.25: Evolution of rotational energy in units of total cluster energy for $W_{0}=0.6$. The epochs of maximum collapse are indicated by arrows. Time is given in units of initial half-mass relaxation time $\left(t_{\text {rhi }}\right)$. 


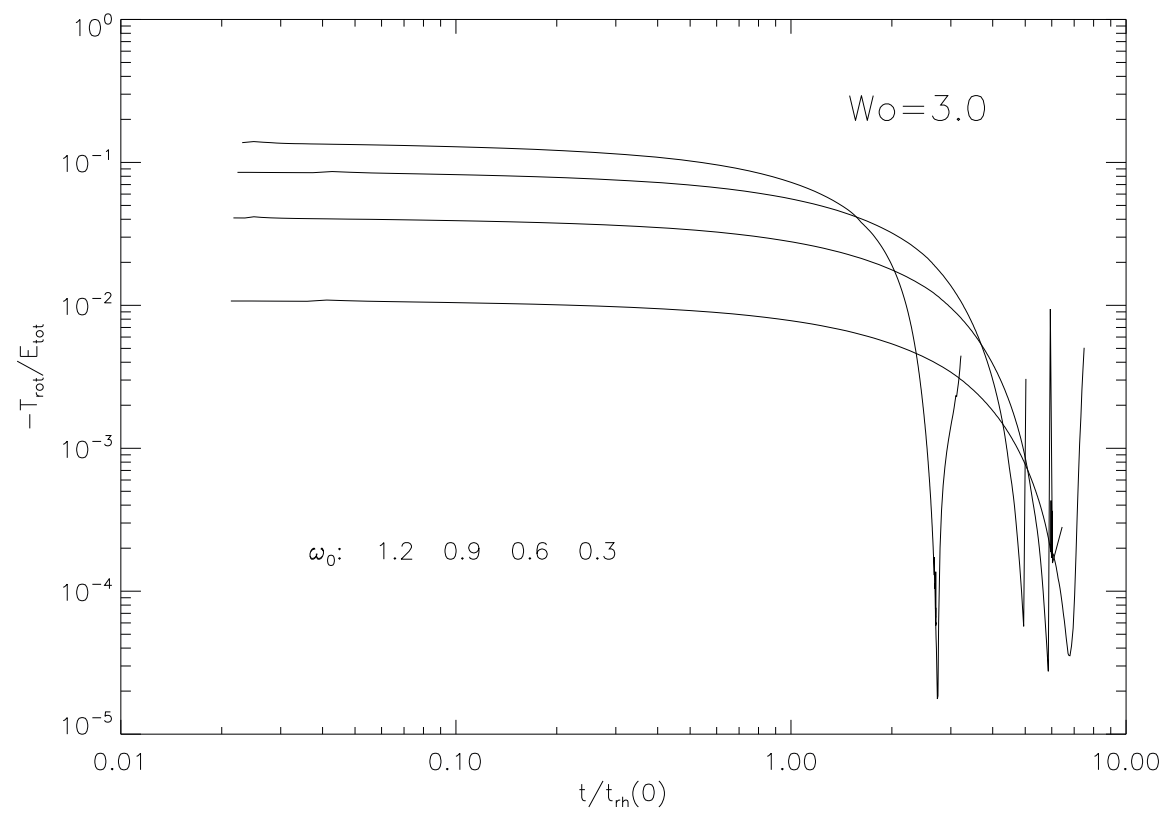

Figure 4.26: Evolution of rotational energy in units of total cluster energy for $W_{0}=0.3$. The epochs of maximum collapse are indicated by arrows. Time is given in units of initial half-mass relaxation time $\left(t_{\text {rhi }}\right)$.

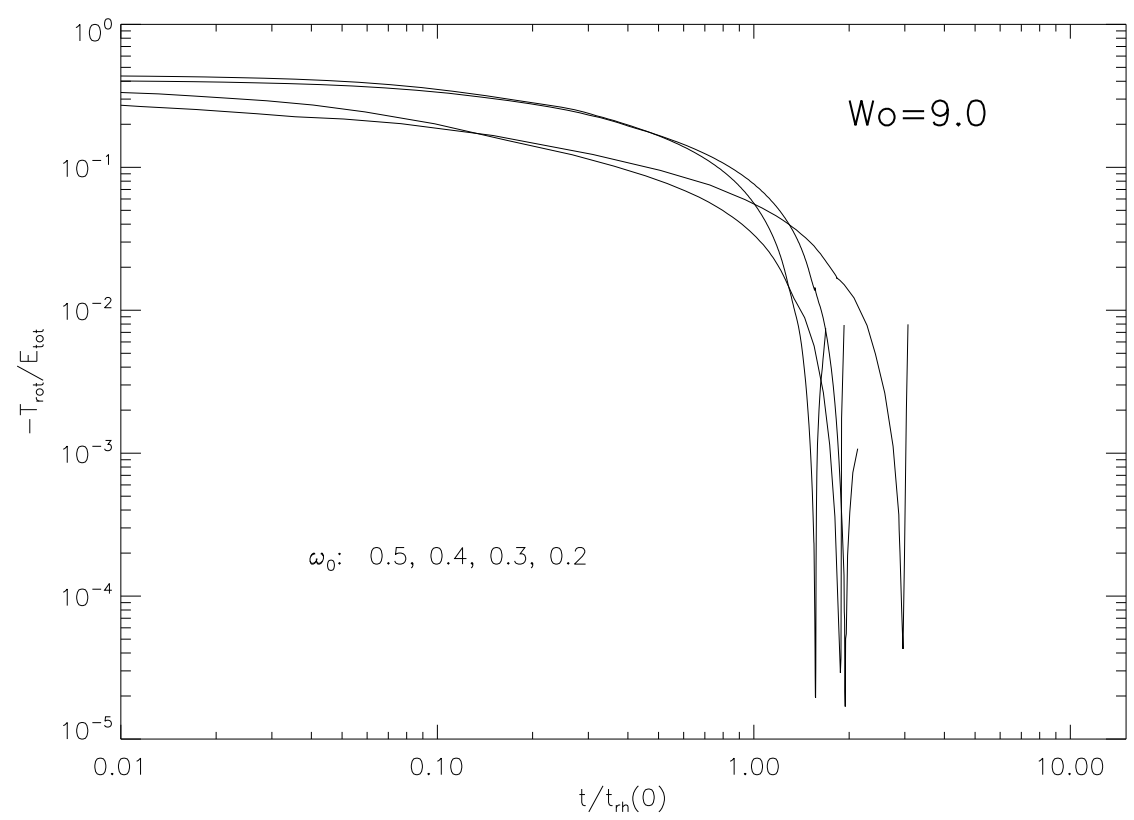

Figure 4.27: Evolution of rotational energy in units of total cluster energy for $W_{0}=0.9$. The epochs of maximum collapse are indicated by arrows. Time is given in units of initial half-mass relaxation time $\left(t_{\text {rhi }}\right)$. 


\begin{tabular}{|c|c|c|c|}
\hline Model name & $t_{\mathrm{cc}} / t_{r h_{i}}$ & $T_{\text {rot }} / E_{\text {tot }}\left(t_{\mathrm{cc}}\right)$ & $e_{\mathrm{dyn}}$ \\
\hline \hline $\mathrm{A} 2$ & 6.04 & 0.01 & 0.0019 \\
\hline $\mathrm{A} 3$ & 5.56 & 0.02 & 0.0013 \\
\hline $\mathrm{A} 4$ & 4.65 & 0.03 & 0.0002 \\
\hline $\mathrm{A} 5$ & 2.71 & 0.004 & 0.0000 \\
\hline $\mathrm{B} 2$ & 8.42 & 0.72 & 0.0072 \\
\hline $\mathrm{B} 3$ & 5.46 & 1.13 & 0.0145 \\
\hline $\mathrm{B} 4$ & 3.48 & 1.18 & 0.0161 \\
\hline $\mathrm{B} 5$ & 1.97 & 1.00 & 0.0164 \\
\hline $\mathrm{C} 1$ & 1.86 & 1.72 & 0.0180 \\
\hline $\mathrm{C} 2$ & 1.42 & 0.95 & 0.0101 \\
\hline $\mathrm{C} 3$ & 1.60 & 1.10 & 0.0153 \\
\hline $\mathrm{C} 4$ & 1.36 & 0.91 & 0.0111 \\
\hline
\end{tabular}

Table 4.6: Rotational parameters at collapse:

$t_{\mathrm{cc}} / t_{r h_{i}}$ : core collapse time in units of $t_{r h_{i}}$;

$T_{\text {rot }} / E_{\text {tot }}\left(t_{\mathrm{cc}}\right)$ : fraction of rotational energy to total system energy.

$e_{\text {dyn }}$ : dynamical ellipticity at collapse time.

Fig. 4.23). $W_{0}=9.0$ models start with a higher rotational energy and ellipticity (Table 4.2), but due to their higher concentration, collapse happens much faster for them. Nevertheless, a certain amount of rotational energy remains in the system at collapse time, comparable or higher than in the other models. $W_{0}=6.0$ models have the longest core collapse times, but they seem to maintain rotation increasing at collapse and short time after collapse (Fig.4.22), leading to a higher rotating collapsed core in comparison with other models, before rotation drops in the post collapse phase. They show also, in general, higher ellipticities.

Evolution of dispersion and rotational velocity analyzed in the last paragraphs can be joined to the rate of rotational velocity over velocity dispersion, which represents the importance of ordered motion in comparison with random motion. Observations about the steep rise of $V_{\text {rot }} / \sigma$ in central regions of globular clusters, mentioned in Sect.(2.3), can not be explained by non-rotating models as by single or multi-mass rotating models without $\mathrm{BH}$. During the evolution of BH-models, a growing of $V_{\text {rot }} / \sigma$ already at early times and a further increasing up to collapse time is observed. This behavior always depends on (a high) rotation initially present in the system and an efficient angular momentum diffusion and $\mathrm{BH}$ star accretion, which are able to lead to an increasing in central rotational velocity before angular momentum is consumed. As seen previously, $W_{0}=3.0$ models exhibit a higher mass loss and do not develop higher core rotation, as it falls very fast after a small increase at the beginning of evolution, with a faster decrease than $\sigma_{\mathrm{a}}$, which also drops with

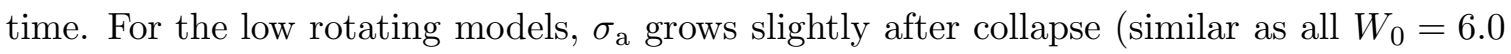
models), while $\Omega$ continues decreasing. As they are clusters with small tidal radii and large cores, mass is easily lost due to an efficient angular momentum diffusion outwards driven by relaxation, starting with a low rotational energy in comparison to other models (see the relaxation times and rotational parameters between models in Tables 4.2 and 4.3). High concentrated $W_{0}=9.0$ models experience generally a faster core collapse, and diffusion is not efficient enough to transport angular momentum in such a short time. As core rotation in $W_{0}=9.0$ models is initially smaller than $\sigma_{\mathrm{a}}$, they develop an initial small rate $V_{\text {rot }} / \sigma$, 
with a moderate growth of rotation and dispersion, during the evolution, leading to a short neto core rotation. On the other side, $W_{0}=6.0$ models are able to maintain an efficient angular momentum diffusion, increasing the core rotation, and at the same time are enough concentrate to avoid an excessive mass loss. Both effects support the accretion of stars in low- $J_{\mathrm{z}}$ orbits and an ordered motion around the $\mathrm{BH}$ at the cluster center. The result is a more stable evolution of $V_{\text {rot }} / \sigma$, with an increase of rotation and decrease of dispersion up to collapse, and a slightly increase of both after collapse time (Figs. 4.15 and 4.22). As in all models, the higher core rotation will depend directly of the initial rotation of the cluster. A comparison between high and low concentrated models shows that higher rotating $W_{0}=3.0$ models lose core rotational velocity also faster, while higher rotating $W_{0}=6.0$ and $W_{0}=9.0$ models retain growing angular velocities in their cores (Fig. 4.24) and comparable high rotating parameters at collapse time (Table 3.27). Nevertheless, due to the instability of the high concentrated $W_{0}=9.0$ models to the gravothermal effect (Quinlan 1996) and less efficient angular momentum diffusion in always shorter collapse times, they do not develop considerable central rotation. The highest central rotation is observed in the $W_{0}=6.0$ models. Moreover, in all models, angular momentum loss accelerates after collapse time and most of the rotation is taken out of the system, leading to a final nearly spherical re-expanding cluster, embedding a central IMBH with a mass of $\sim 1 \% M_{\mathrm{cl}}$.

In Fig. (4.28) the $V_{\text {rot }} / \sigma$ curve corresponding to Fig. (4.21d) is presented. It shows the state of the initially high rotating model B4 $\left(6.0,0.9,5 \cdot 10^{-6}\right)$ at time zero (dot-dashed line) and at the time of maximum of core rotation (solid line) taken from evaluation of the evolution of angular velocity shown in Fig. (4.29) before angular momentum loss accelerates, i.e. before collapse is reversed. The initial rotational profile, shows a maxima at approximate the half-mass radius and evolution makes clear its decrease and short approximation to the center, while in the center counter-rotation rises. A comparison of these result with the central $V_{\text {rot }} / \sigma$ profile of the galactic GC M15 (Gebhardt et al. 2000; Gerssen et al. 2002, 2003) shows a comparable rate $V_{\text {rot }} / \sigma \lesssim 1$ as the reported by these authors from observational studies (they found a maximum of $V_{\text {rot }} / \sigma \sim 1$ ). Moreover, M15 is not the only GC where high central rotation has been found. Another examples are the galactic GCs M4, M5, M80, NGC2808 and NGC5286 (Gebhardt, K. personal communication). As previously discussed, the further evolution exhibits a decreasing in the central rotation, as well as in the outer regions, when the cluster becomes close to spherical and finally dissolves, leaving an IMBH behind, which orbits its parent galaxy.

In Fig. (4.29), the evolution of rotation in the respective mass shells are plotted. The shells closer to the center experience a rapid increase of rotation close to the collapse, an a further decrease later. The rotation increase is always smaller when the mass shells contain more fractions of mass. In the outer shells, rotation is lost very fast, as angular momentum is carried away. Angular momentum transport is very efficient at early times of evolution, as can be seen in Fig. (4.30), specially in the core, where the specific angular momentum is depleted stronger for the innermost mass shells than for the outer shells. No significant visible transfer of angular momentum to the outer shells during the evolution is observed, as angular momentum is also consumed at the center by the BH, while gravogyro instabilities lead to further mass loss through the galactic tidal boundary. As mentioned, energetic eccentric orbits dominate the halo and core, which can be rapidly lost from the system through tidal disruption or $\mathrm{BH}$ star accretion. 


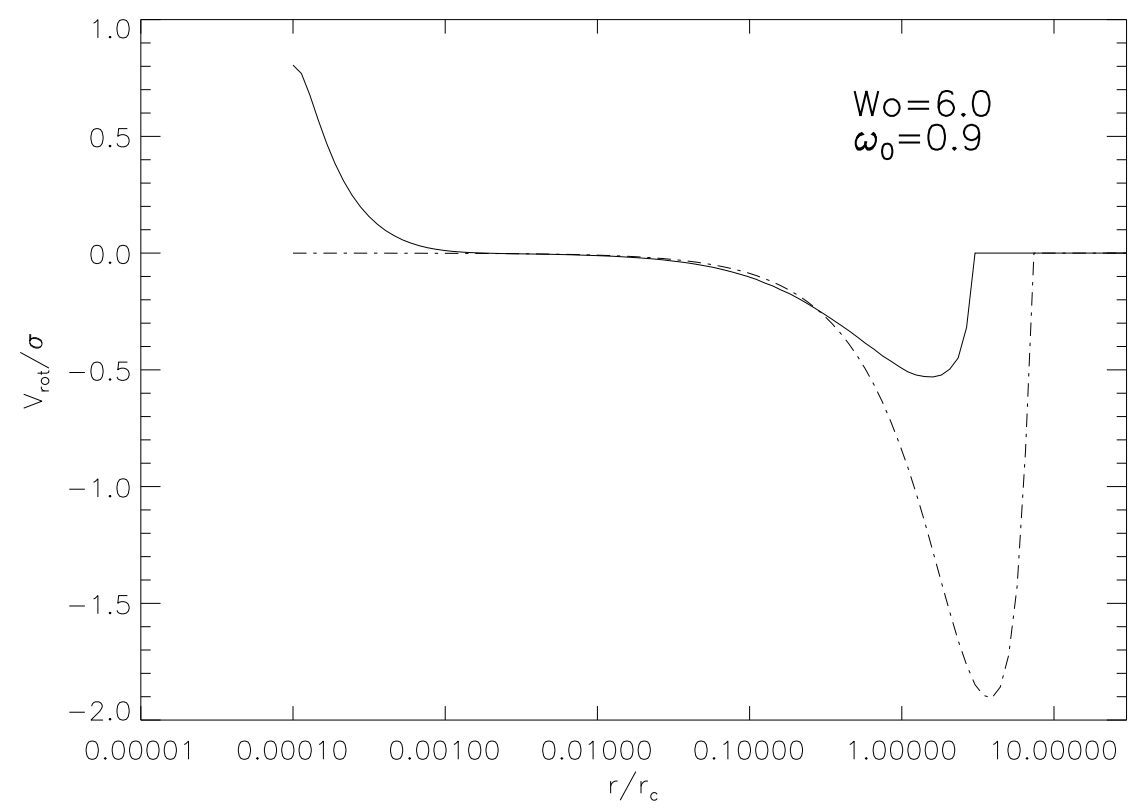

Figure 4.28: $V_{\text {rot }} / \sigma$ as a function of radius in units of initial core radius for the model B3 $(6.0,0.6,5$. $\left.10^{-6}\right)$ at collapse time. The dotted-dashed line represents the initial profile. The location of the initial maximum $V_{\text {rot }} / \sigma$ moves inward and drops in time. A second maximum grows in the core and in opposite direction of initial rotation

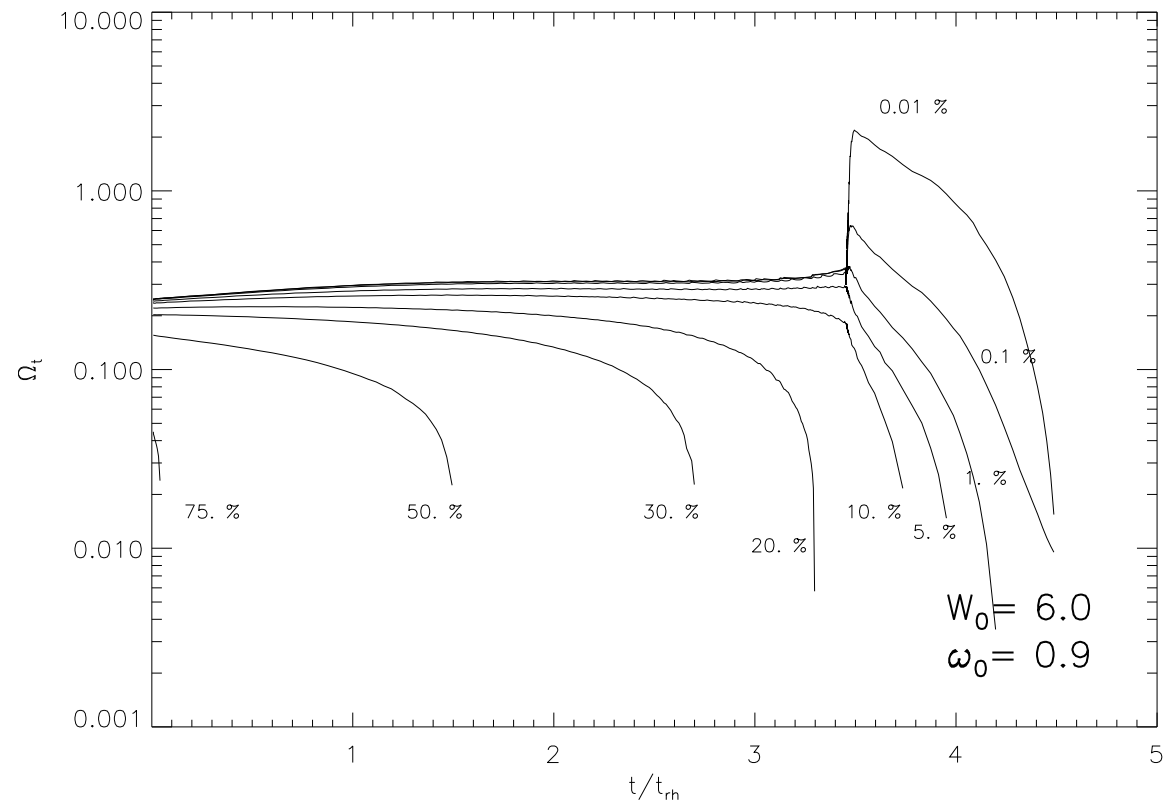

Figure 4.29: Evolution of angular velocity in cylindrical shells containing the masses indicated in the figure for the model B4 $\left(6.0,9.0,5 \cdot 10^{-6}\right)$. Time is given in units of initial $t_{\mathrm{rh}}$. 


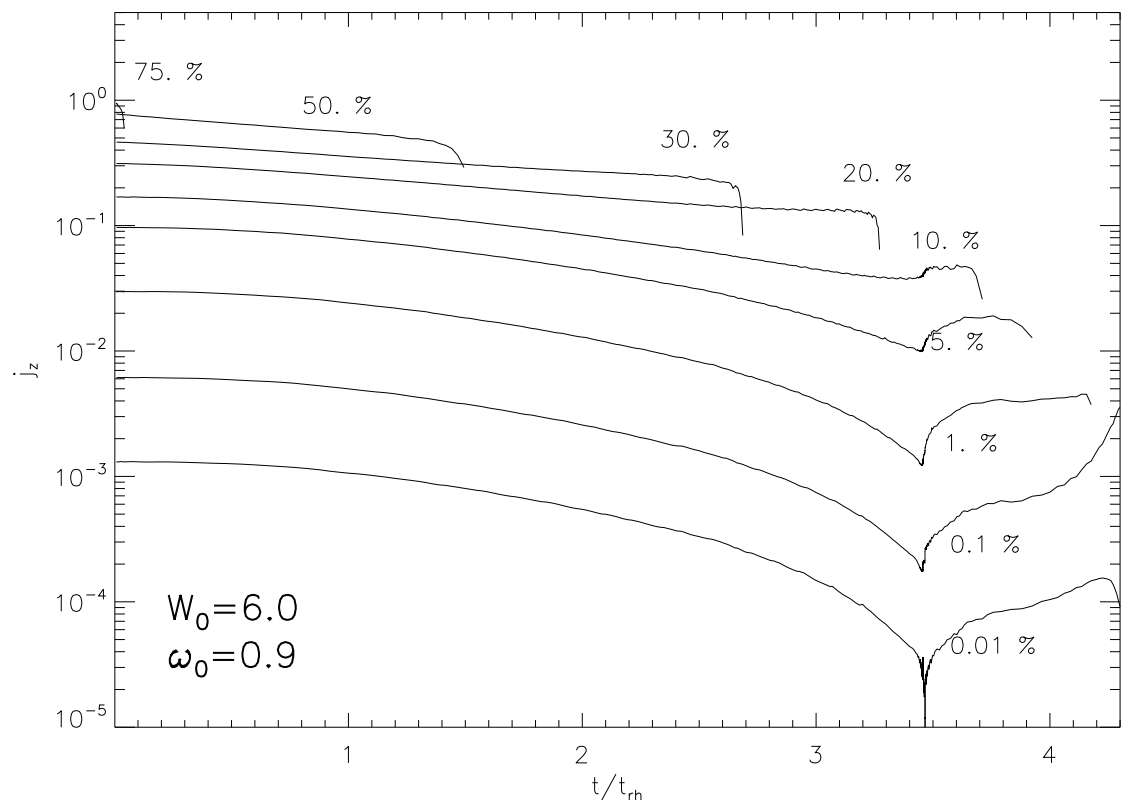

Figure 4.30: Evolution of specific angular momentum in cylindrical shells containing the masses indicated in the figure for the model B4 $\left(6.0,9.0,5 \cdot 10^{-6}\right)$. Time is given in units of initial $t_{\mathrm{rh}}$. 
The results presented here support the thesis that the presence of a central dark mass, could explain the observations of higher central rotation in GCs. The higher central $V_{\text {rot }} / \sigma$ is, nevertheless, not more present at later evolutionary times, when angular momentum is near completely carried away from the system. In this sense, according to the present study, the central slope in the $V_{\text {rot }} / \sigma$ profile, does not represent a steady state, like the density or velocity dispersion profiles, which central cusps remain approximately unchanged after collapse, and extend their radius of influence as $M_{\mathrm{BH}}$ grows. On the other side, the simplified conditions of the present simulations listed in Sect. 3.1.1 make clear the necessity of including a mass spectrum and stellar evolution in the models, as well as a more realistic criteria for galactic tidal mass loss. Comparison to N-Body models are aimed to complement this calculations, using the highest particle number permitted at the time $\left(\sim 10^{6}\right)$. Regarding the stellar spectrum, the distribution of high mass stars is expected to drop the dispersion in the center (as reported by Kim et al., 2004), leading to a higher $V_{\text {rot }} / \sigma$ in this regions. Nevertheless, the agreement of this profile with observations and the consistence of the general evolution of cluster structure including an embedded BHs the systems with current studies, without rotation, makes clear that rotation constitutes an important constraint, which needs to be taken into account in the understanding of the formation and evolution of GCs, specially when rotation is high enough, at early times (e.g. in the young clusters of the LMC).

\subsection{Distribution function}

Rotating models without $\mathrm{BH}$ are known to show deviations from the initial King distribution $f\left(E, J_{z}, t=0\right)$, at times up to the collapse and later (Einsel 1996, 1999). The initial angular velocity parameter $\Omega_{0}$, i.e. the rotation, can be messed in the constant slope of $f\left(J_{z}\right)$, at constant energy $E$ (Fig. 4.1). Evolution of rotating clusters without BH shows a flattening of the $f\left(J_{\mathrm{z}}\right)$ curves at higher energies at later times, since $J_{\mathrm{z}}$ is driven away from the system.

Evolution of the angular momentum averaged distribution function $f(E)$ using the current BH models, is shown in Fig. (4.31) for different times (in parenthesis). The cusp is initially depleted and forms slowly a canonical cusp close to collapse, when $f(E)$ approximates the 1/4 - slope of the equilibrium solution (Bahcall \& Wolf, 1976) at energies deeper than $\left|E_{\text {crit }}\right|\left(\simeq 0.9\right.$ for the last profile at $t / t_{\mathrm{rh}_{\mathrm{i}}} \simeq 3.5$, broader solid line) falling steeper at higher energies, where the angular momentum dependence of $f\left(E, J_{z}\right)$ becomes significant, due to the importance of the loss-cone ( $E_{\text {crit }}$ was defined in Sect. 3.2.2). As mentioned also in this section, the number density of stars at higher energy, low $J_{\mathrm{z}}$ loss-cone orbits does not drop to zero. Moreover, star accretion is a direct consequence of angular momentum diffusion to the central region close to the BH. Evolution shows a broadening of $f(E)$ in time (dot-dashed curves) as the energy range of stellar orbits grows with the central potential cusp of the BH.

Figure (4.32) shows the two-dimensional distribution function in the $\left(E, J_{\mathrm{z}}\right)$-plane at time of maximum of rotation, close to collapse, for the same model of Fig. (4.31), using model B4. The curves of constant energy are shown with their respective energy values listed in the left columns. The z-component of angular momentum is given in units of $J_{\mathrm{z}}^{\max }$, in order to make visible the profiles at higher energies, where $J_{\mathrm{z}}$ becomes very small. The figure is separated in two panels, to avoid overlapping of the curves at higher energies, 


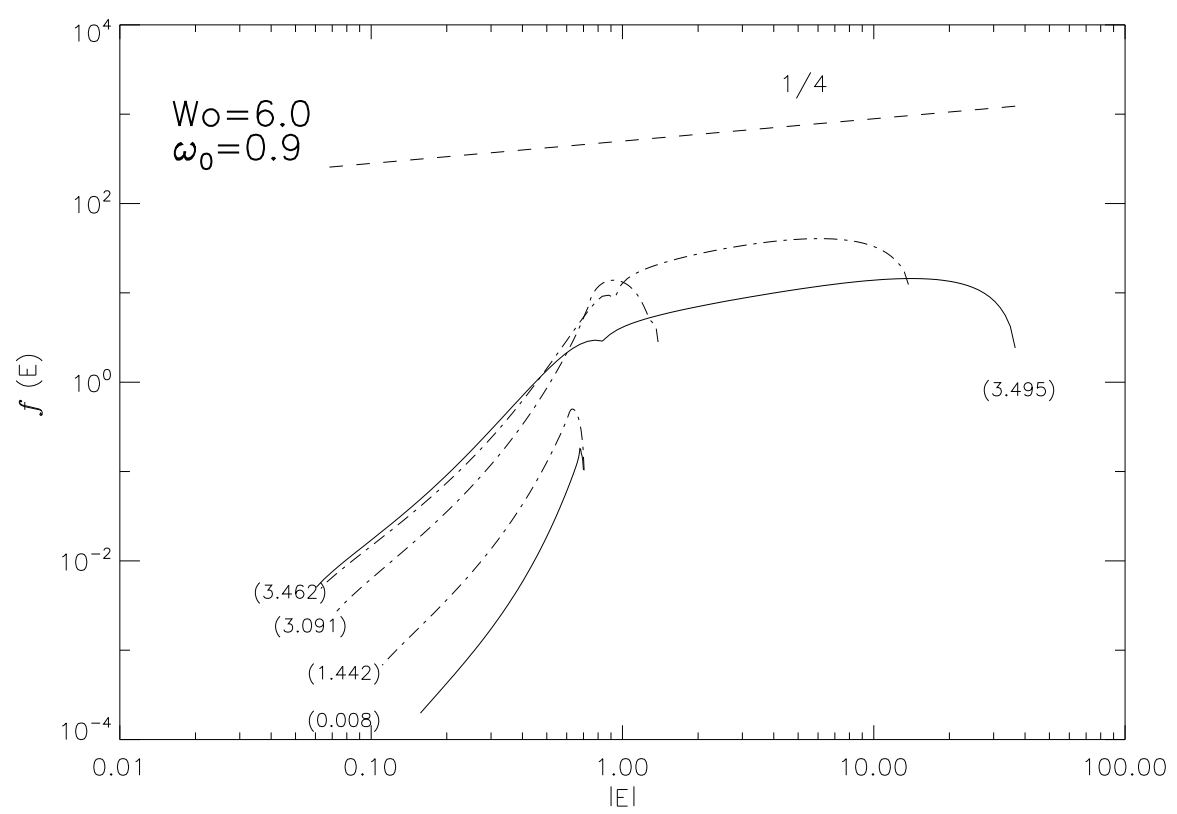

Figure 4.31: Evolution of angular momentum averaged distribution function for a cluster with a final $M_{\mathrm{bh}} \simeq 0.01 M_{\mathrm{cl}_{1}}\left(\simeq 5000 M_{\odot}\right)$. The dashed-line shows the $1 / 4$-slope of the equilibrium solution. The initial and final profiles are shown through solid curves (the final state is the broader one), the dot-dashed curves show the evolution of the profiles. $E_{\text {crit }} \simeq 0.9$ in the last curve, at $t / t_{\mathrm{rh}_{\mathrm{i}}} \sim 3.5$ 
where the effects of loss-cone accretion depletes $f\left(E, J_{z}\right)$ specially for stars in low- $J_{z}$ orbits, clearly visible in the depth around $J_{\mathrm{z}}=0.0$, leading to negative anisotropy, as circular orbits dominate over radial orbits, preferably depleted through $\mathrm{BH}$ accretion. This was shown in Fig. (4.8), in the radial profile of anisotropy $\left(A \equiv 1-\frac{\sigma_{\phi}{ }^{2}}{\sigma_{\mathrm{r}}{ }^{2}}\right)$ inside the BH influence radius, which extends as $M_{\mathrm{BH}}$ grows, and in the profiles of velocity dispersion components $\sigma_{\phi}$ and $\sigma_{r}$ (Fig. 4.7). On the other side, the outer regions of the system develop positive (radial) anisotropy (although many times smaller than the dominant negative), because unbounded circular orbits are expelled easily from the cluster through the tidal boundary. At the same time, high energy radial orbits begin to dominate the cusp, under the influence of the central potential cusp. Later, as mass is lost from the system due to energy and angular momentum transport, eccentric orbits will be lost through the galactic tidal boundary, and negative anisotropy will outbalance in all regions.

As discussed in the previous section, orbits with positive $J_{\mathrm{z}}$ dominate the high energy region, leading to higher central positive rotation, while negative anisotropy can be seen in the central depth of the same energy curves. Isotropy and no rotation is observed at mid energies, where the distribution function does not show a strong $J_{\mathrm{z}}$-dependence. On the other side, orbits of negative $J_{z}$ dominate the low-energy region (lower curves in panel below) but the amount of rotation is much less than the initial configurations by some orders of magnitude (see Fig. 4.1 for comparison). The snapshot is taken from the evolution of model B4, at collapse time $\left(t_{\mathrm{cc}} / t_{\mathrm{rh}_{\mathrm{i}}} \sim 3.5\right)$, where $E_{\text {crit }} \simeq 0.9$.

Evolution of two dimensional distribution $f\left(E, J_{\mathrm{z}}\right)$ for three different models is shown in Figs.(4.33) to (4.35). The angular momentum is shown in units of $J_{\mathrm{z}}^{\max }$ (Eq. 3.12) for a better representation in the plane. Energy is given in code units, using Eq. (3.11). As in Fig. (4.31), distribution of stars covers a wider area in time, as the BH cusp grows and energy of bounded stellar orbits increases. After collapse time (lower right panels in all figures) the distribution is nearly symmetric with respect to the $Y=0$-line, i.e. no significant rotation is present in the system. Nevertheless, during the evolution towards collapse, when star accretion dominates and rotation is growing in the zone of influence of the $\mathrm{BH}$, $f\left(E, J_{z}\right)$ develops an observable asymmetry in its distribution at high energies (e.g. Fig. $4.33 \mathrm{~b}, \mathrm{c}$ ), with higher number density of stars in orbits of positive angular momentum (at $Y \lesssim+1$ ). At earlier times, an asymmetry in the direction to lower energies (more stars in negative $J_{z}$ orbits) is observed (Fig. 4.33a). At later times, $f\left(E, J_{\mathrm{z}}\right)$ achieves the steady state solution showed in Fig. (4.31), while rotation drops and the cluster becomes spherical.

As mentioned in the last section, intermediate models evolve to a more stable increasing of central rotation, driven by the interplay of efficient angular momentum diffusion and $\mathrm{BH}$ accretion of stars in low- $J_{\mathrm{z}}$ orbits. The effect is stronger, the higher the initial rotation of the model. On the other side, initially low concentrated $\left(W_{0}=3.0\right)$ models build hardly a high rotating core. Even the highest rotating model A5 here presented can not form a considerable higher asymmetry in the distribution of $f\left(E, J_{\mathrm{z}}\right)$ (Fig. 4.34), leading to a lower maxima of rotation in this models at always shorter collapse times with increasing initially rotation parameter. In high concentrated models $\left(W_{0}=9.0\right)$, the rapid evolution (the shortest collapse times), leads to an inefficient transport of angular momentum through diffusion, and an unsupported $\mathrm{BH}$ mass growth through rotation and vice-versa, and as a consequence, a faster depletion of central rotation (Fig. 4.35). In the latter models, the 

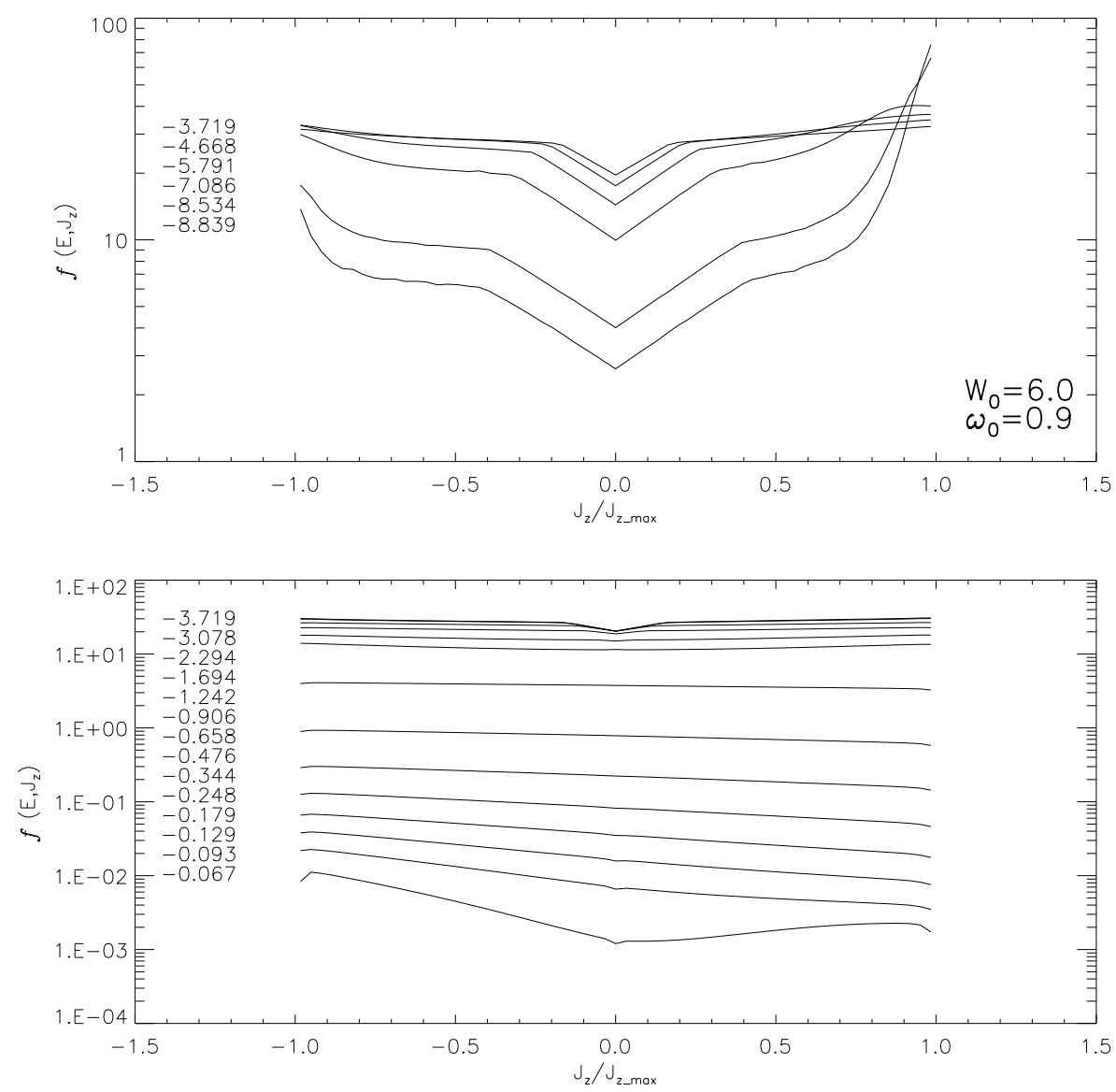

Figure 4.32: 2 Dimensional distribution function in $\left(E, J_{z}\right)$-plane. Shown are the curves of constant energy (values listed in the left columns). The figure is separated in two panels, to make clear the $J_{\mathrm{z}}$-dependence of the distribution function specially at higher energies, due to loss-cone accretion of stars in low- $J_{\mathrm{z}}$ orbits. $E_{\text {crit }} \simeq 0.9$ and the time is the collapse time of the model B4, $t_{\mathrm{cc}} / t_{\mathrm{rh}_{\mathrm{i}}} \sim 3.5$. Isotropy would require a constant $f$, for all $J_{\mathrm{z}}$. 
maxima of rotation is, consequently, achieved earlier, when the angular momentum source in the core is still not large enough to support evolution of rotation during the further evolution up to post-collapse phase. The final distribution of $f\left(E, J_{\mathrm{z}}\right)$ in this models, shows a positive (radial) core anisotropy, driven by the inefficient angular momentum transport and the smallest cluster mass loss, with the implication that radial orbits are retained in the cluster more than in the less concentrated models.

As a conclusion, the amount of rotation present in the system during its dynamical evolution is a result of the interplay between diffusion of angular momentum (leading to mass loss due to the galactic tidal field) and the redistribution of high energy orbits close to the $\mathrm{BH}$ (through accretion of most radial orbits and development of ordered motion in its zone of influence). In this sense, the central BH affords the formation of an angular momentum source near the center, which supports gravogyro instabilities leading to core collapse. In a further speculation, as the central BH is able to 'consume' angular momentum of the system (in fact, it accretes continuously stellar mass with an specific amount on $J_{\mathrm{z}}$ ), it can become an angular momentum source itself, which could be able to rotate (Kerr Black Hole), permitting also a more efficient angular momentum transport outwards, through interaction with core stars, driven by the gravogyro instability. The circumstance of a binary black hole (BBH) system, could, in a similar way, lead to a more efficient support in the development of central rotation, modifying substantially the final shape of the cluster. Angular momentum transport outwards is enhanced by these effects, accelerating core collapse, with respect to rotating systems without BH. The BH acts as well as an energy source, that drives post-collapse evolution, while mass loss rate increases until the cluster dissolves, leaving behind an IMBH, which orbits around the parent galaxy.

As mentioned in the previous section, the presence of different mass components in real clusters is also an important factor to be considered in the distribution of number density of stars. In multi-mass models without BH (Kim et al. 2004), around 2 times faster rotation was observed in the high-mass components (which concentrate in the central part of the system due to mass segregation) with respect to low-mass components. Nevertheless, angular momentum decreases continuously through the whole evolutionary phase. This additional effect could increment the central rotation growth found with the current BH-models.

As discussed in Sect. (1.3), the cluster shape (i.e. ellipticity) is a consequence of the distribution of rotation in globular clusters. In Figs. (4.36) and (4.37), evolution of dynamical ellipticity (as defined in Eq. 3.36) is shown. The rapid decay of ellipticity close to the collapse is observed in all rotating models. It is faster, as collapse happens earlier. At collapse time (arrows in Fig. 4.37), no cluster has an ellipticity larger than 0.05. Due to the reverse of collapse and cluster expansion during the post-collapse phase, the ellipticity seems to increase again in a small amount. A comparison with Fig. (4.25) makes clear its origin in the rotational energy of the system.

As seen in the figures, higher ellipticities are initially present at high concentrated models with the same rotation. During the evolution, low concentrated and low rotating models develop lower final ellipticities in shorter times, than higher concentrated models with the same rotation. If the initial rotation is higher, $W_{0}=3.0$ models tend to lose their elliptical shapes in longer times than $W_{0}=6.0$ models with the same initial (high) rotation, and the collapse times are longer than that of the latter models (compare Table 4.6). Thus, high 
a) $t / t_{\text {rhi }}=1.43$

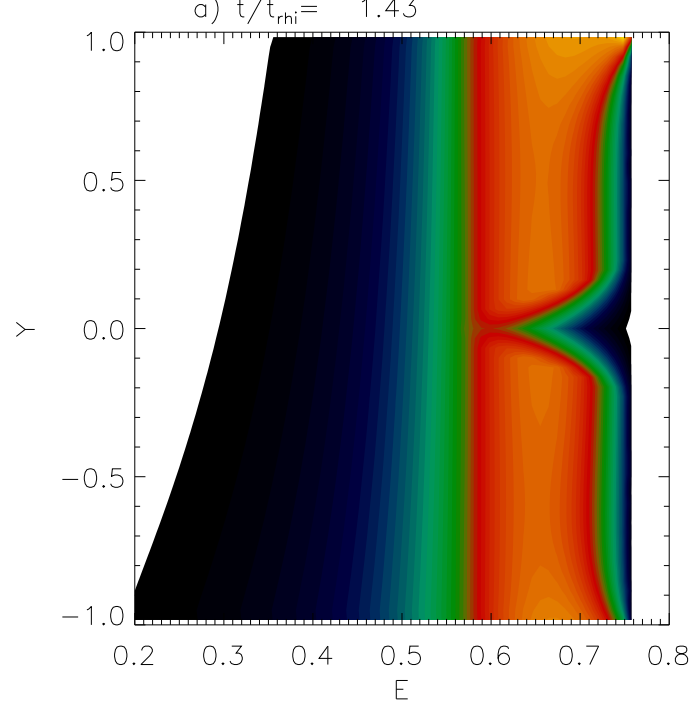

c) $t / t_{\text {rri }}=1.97$

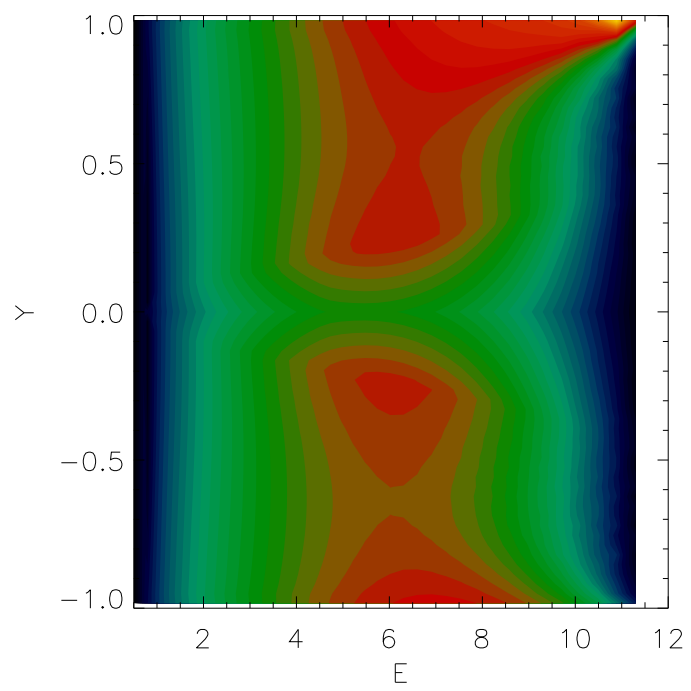

b) $t / t_{\text {rhi }}=1.84$

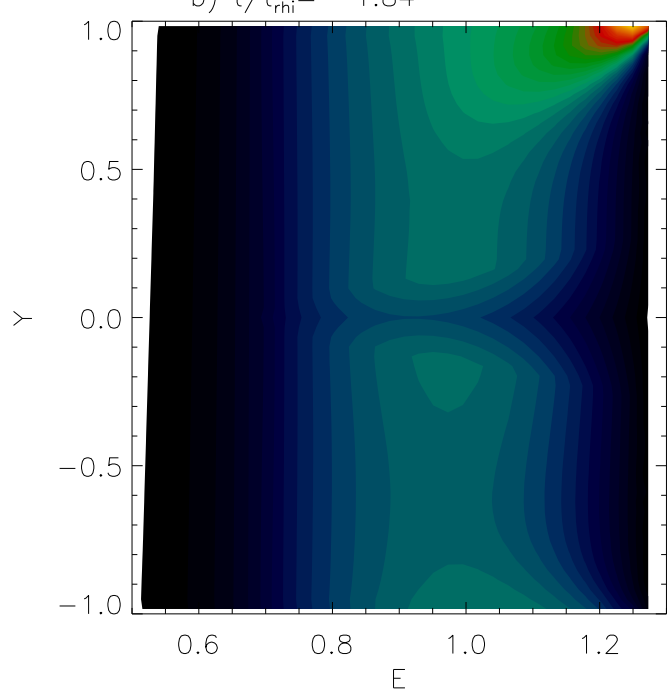

d) $t / t_{r h i}=2.14$

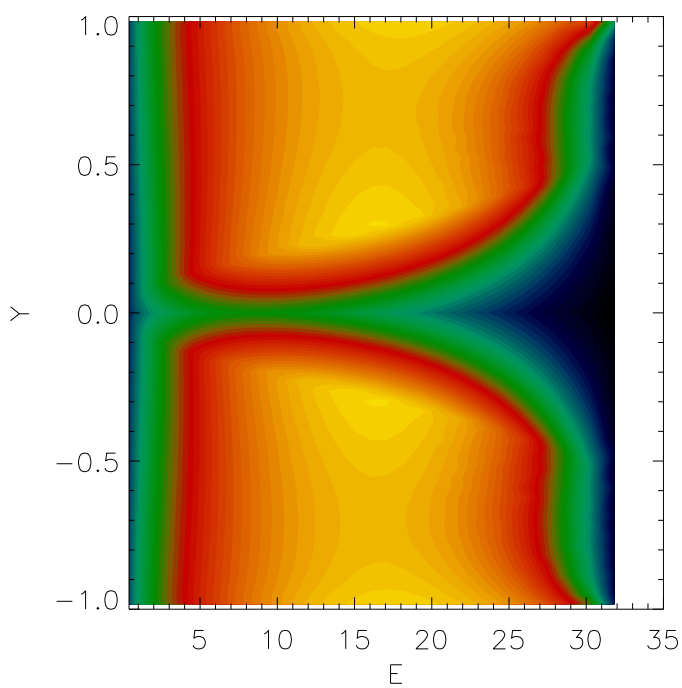

Figure 4.33: Evolution of distribution function in the $E-Y$ plane for the model B5 $\left(6.0,1.2,5 \cdot 10^{-6}\right)$. The time is given in units of initial half-mass relaxation time $\left(t_{\mathrm{rhi}}\right)$. 

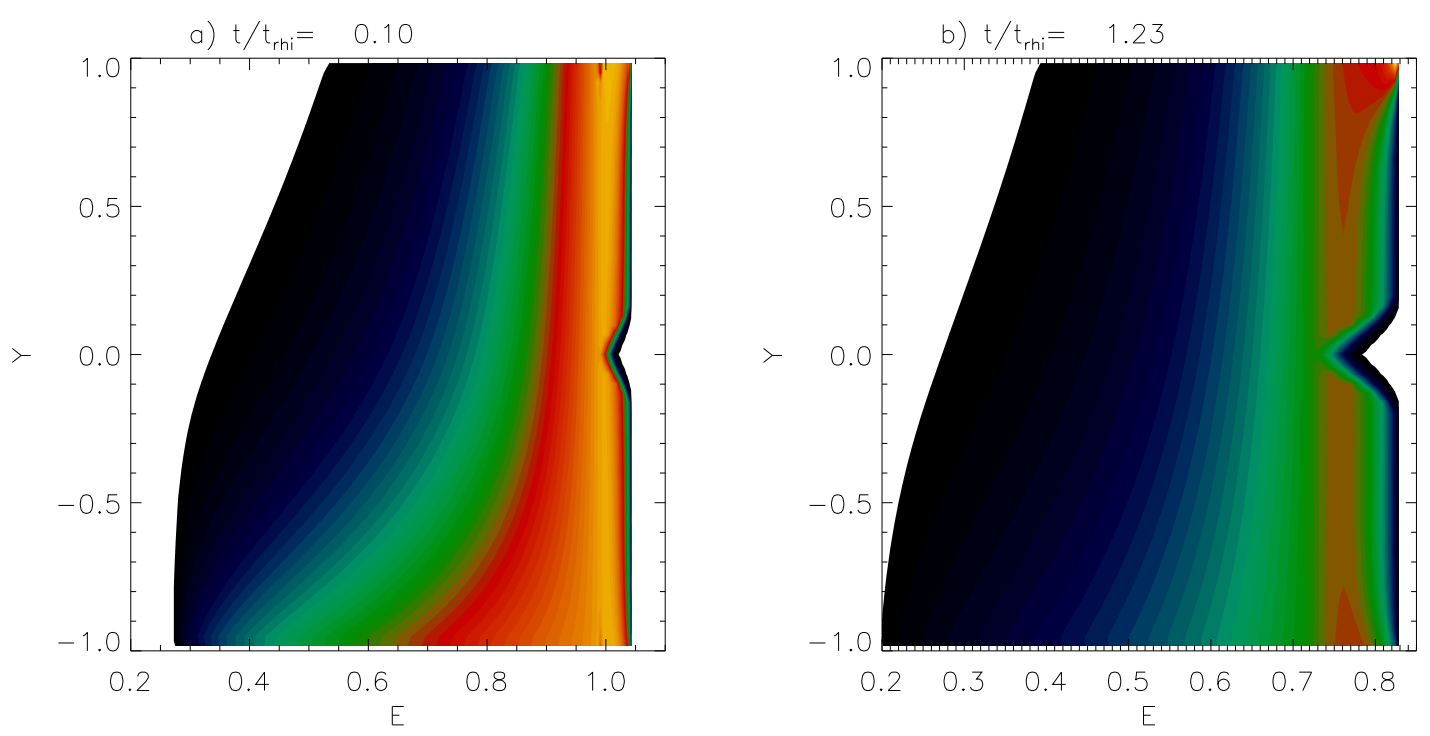

c) $t / t_{\text {rri }}=1.92$

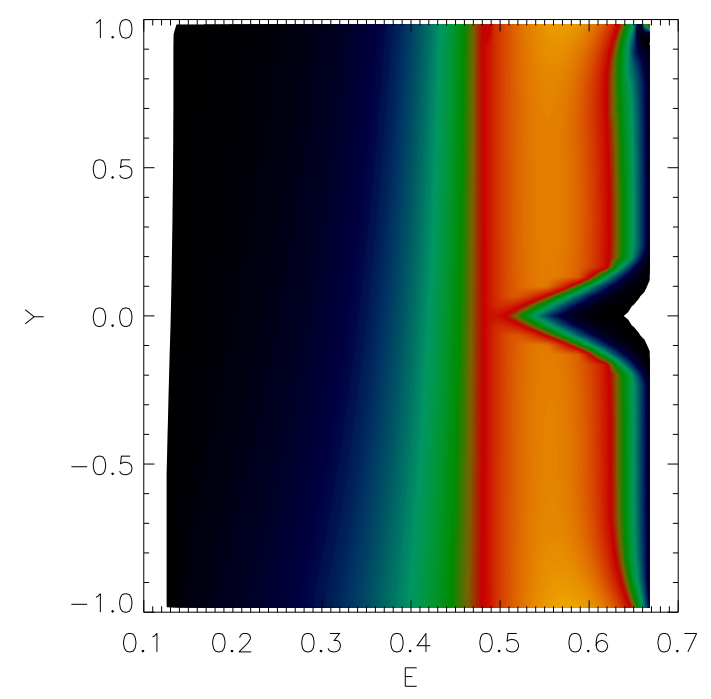

d) $t / t_{\text {rhi }}=2.76$

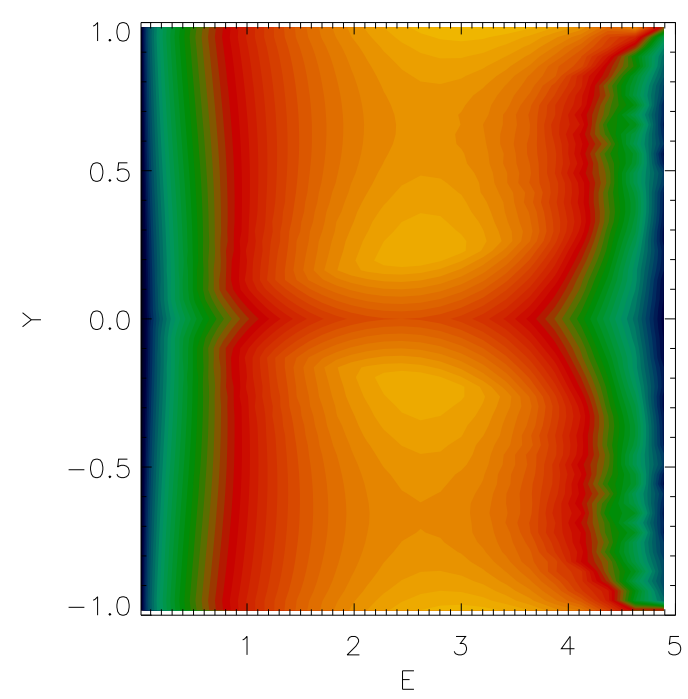

Figure 4.34: Evolution of distribution function in the $E-Y$ plane for the model A5 $\left(3.0,1.2,5 \cdot 10^{-6}\right)$. The time is given in units of initial half-mass relaxation time $\left(t_{\mathrm{rhi}}\right)$. 
a) $t / t_{r h i}=0.50$

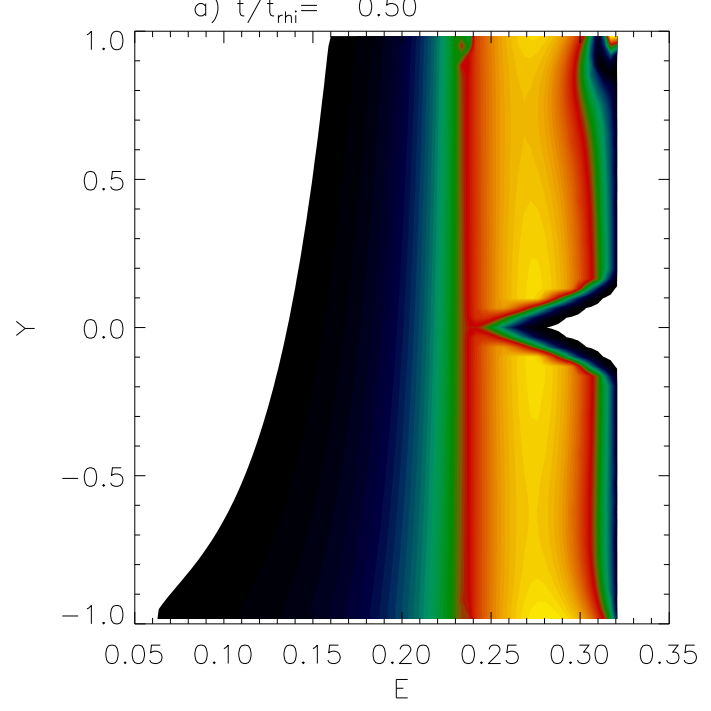

c) $t / t_{r h i}=1.50$

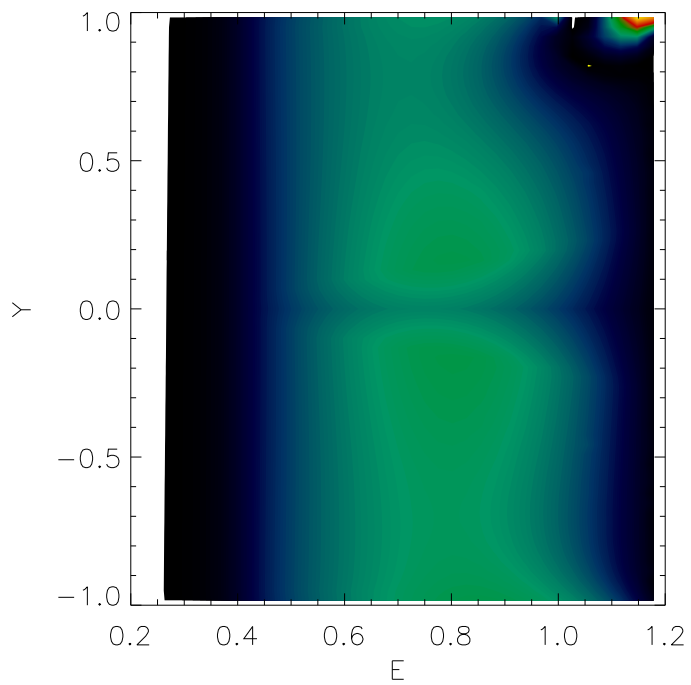

b) $t / t_{\text {rhi }}=0.93$

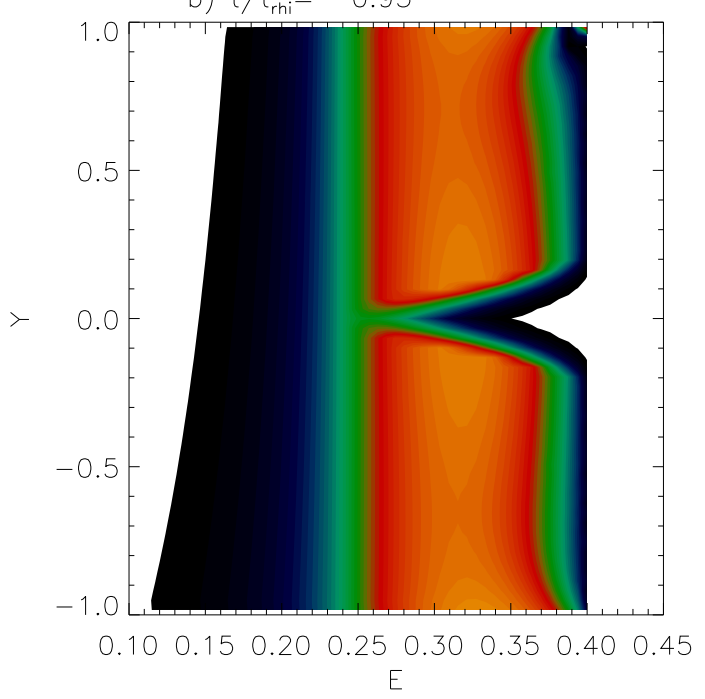

d) $t / t_{r h i}=1.95$

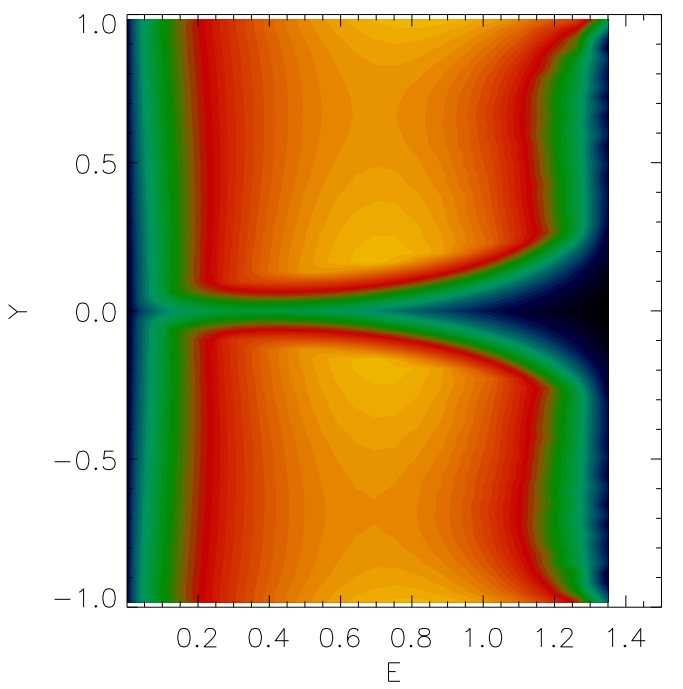

Figure 4.35: Evolution of distribution function in the $E-Y$ plane for the model C3 $\left(9.0,0.4,5 \cdot 10^{-6}\right)$. The time is given in units of initial half-mass relaxation time $\left(t_{\mathrm{rhi}}\right)$. 
initial rotation delays collapse in low concentrated models in a more efficient way than in concentrated clusters. As cluster mass is severely lost after collapse, and lies below the BH mass in a relatively short time, the cluster shape during core expansion is dominated by high energetic bounded orbits to the $\mathrm{BH}$.

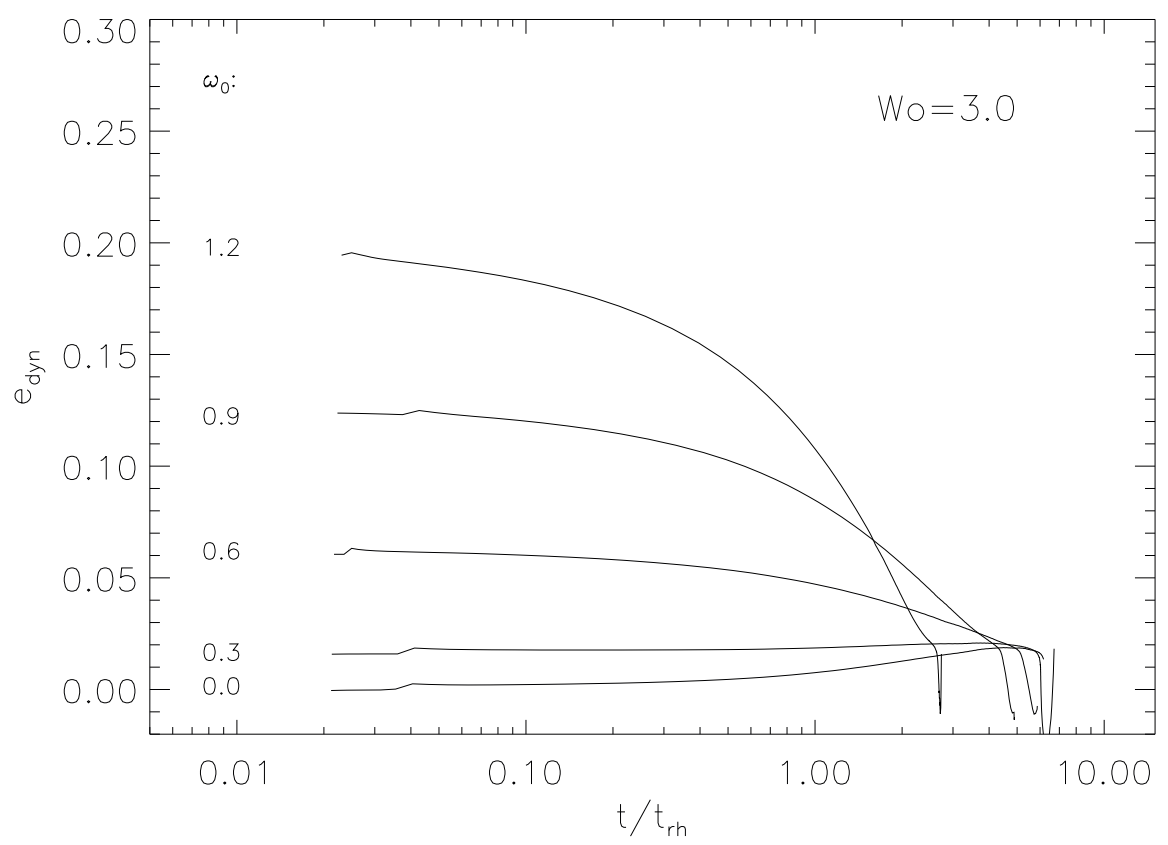

Figure 4.36: Evolution of dynamical ellipticity for $W_{0}=0.3$. The epochs of maximum collapse are indicated by arrows. Time is given in units of initial half-mass relaxation time $\left(t_{\mathrm{rhi}}\right)$. 


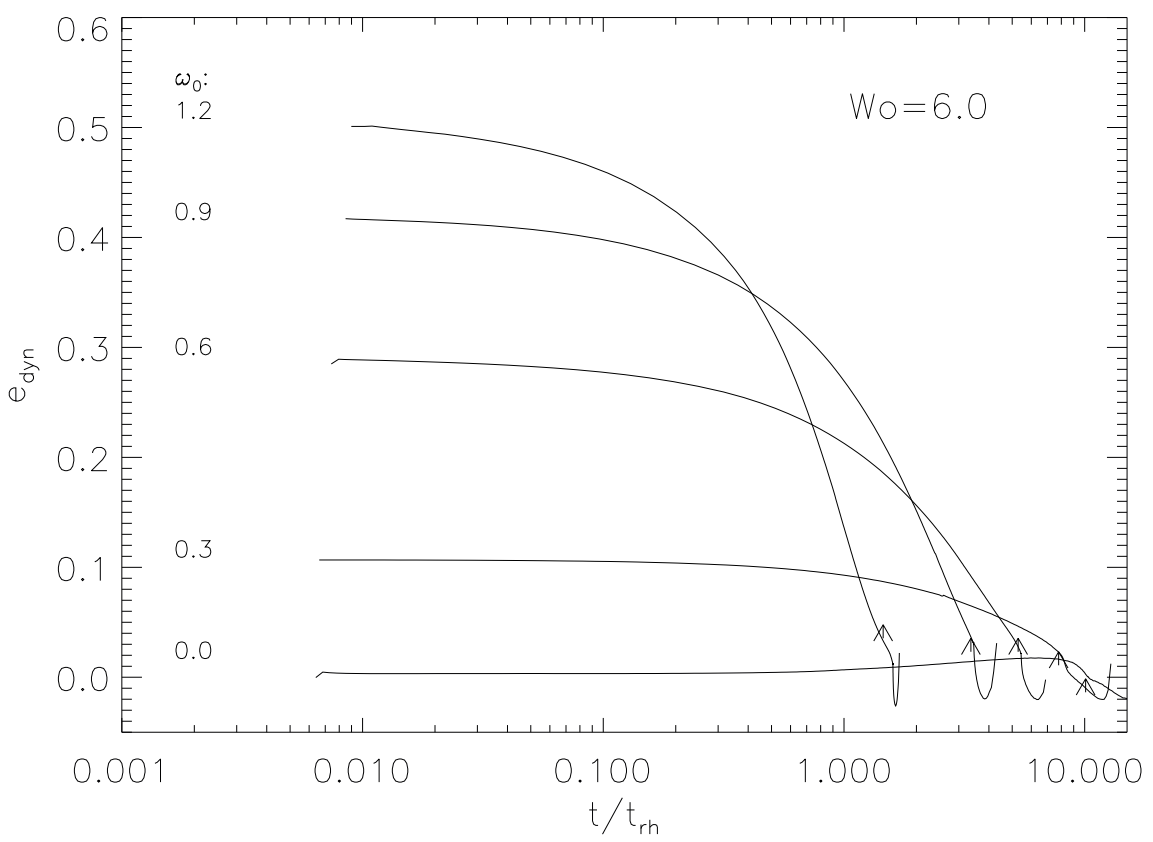

Figure 4.37: Evolution of dynamical ellipticity for $W_{0}=0.6$. The epochs of maximum collapse are indicated by arrows. Time is given in units of initial half-mass relaxation time $\left(t_{\mathrm{rhi}}\right)$. 


\subsection{Observational data comparison}

Due to their higher central densities and concentrations, core-collapse clusters are obvious places to look for central BHs. Kinematical studies have given indications of the existence of central dark objects in GCs, which could explain observations (Gerssen et al. 2002, 2003; Gebhardt et al. 2000, 2002; Miller Colbert 2004). The present work gives theoretical constraints in the evolution of this objects under the assumption of the formation and evolution of a central dark object, and formulates predictions about the evolutionary states of such systems. The models presented, are able to reproduce 2D distributions (in the meridional plane) of density, cluster- and BH-potential, velocity dispersions, rotational velocity, anisotropy, dynamical ellipticity, among other parameters, at any time of evolution. It makes possible to study the evolution of kinematical and structural parameters (radii, time scales, concentration, ellipticity), which complement observational measurements. As mentioned in Sect. (1.4) observational studies of suspected globular clusters with BHs include galactic and extragalactic cluster types, which can be compared to evolutionary models, to elucidate theoretical predictions and understand the common evolution between star clusters and their parent galaxies. With this aim, a detailed set of model data, covering a wide range of rotation rates and initial concentrations of rotating GCs, with and without $\mathrm{BH}$, has been created, which is aimed to enable observers to use them for comparisons with their data.

As mentioned before, the theoretical data obtained with this models, can be rescaled to physical units, by fixing the radius and total mass of the cluster. An application is presented in Fig. (4.38), where the distribution of rotational velocities in the meridional plane $(\varpi, z)$, as well as for the total velocity dispersion are shown (Figs. 4.38a,b). The lower panels (Figs. $4.38 \mathrm{c}$,d) show a zoom of the central region. The distribution of $v_{\text {rot }}$ exhibits an increase of central rotation in form of torus-like contours, and, as can be seen, the formation of central maxima in the equatorial plane. $\sigma_{t}$ builds higher contour levels towards the center, corresponding to Keplerian orbits bounded to the central mass. The snapshot is taken from a collapsed model B4 $\left(6.0,0.9,5 \cdot 10^{-6}\right)$, assuming an initial total cluster mass of $5 \cdot 10^{6} M_{\odot}$ and a core radius of 10 parsec. Velocities are given in $\mathrm{km} / \mathrm{sec}$ and radii in parsec. The final $\mathrm{BH}$ mass is $M_{\mathrm{bh}} / M_{\mathrm{cl}_{\mathrm{i}}} \sim 0.01\left(5 \cdot 10^{4} M_{\odot}\right)$. These contour maps of rotation can be well compared with observational studies like the presented by Meylan \& Mayor (1986) and Merrit et al. (1997) for galactic globular clusters.

In this units the dynamical time (Eq.1.5) is given by:

$$
t_{\mathrm{dyn}} \propto\left(2.34 \cdot 10^{2} \mathrm{yr}\right)\left(\frac{M_{\mathrm{cl}_{\mathrm{i}}}}{10^{6} M_{\odot}}\right)^{-1 / 2}\left(\frac{r_{\mathrm{h}_{\mathrm{i}}}}{10 p c}\right)^{3 / 2}
$$

where $M_{\mathrm{cl}_{\mathrm{i}}}$ represents the initial total mass of the cluster, and $r_{\mathrm{h}_{\mathrm{i}}}$ the initial half mass radius.

The half-mass relaxation time (Eq.1.4) is given by:

$$
t_{\mathrm{rh}} \propto\left(1.414 \cdot 10^{6} \mathrm{yr}\right)\left(\frac{M_{\mathrm{cl}_{\mathrm{i}}}}{10^{6} M_{\odot}}\right)^{1 / 2}\left(\frac{r_{\mathrm{h}_{\mathrm{i}}}}{10 p c}\right)^{3 / 2}
$$

The contour maps presented in Fig. (4.38) were taken at an evolutionary time of $t_{\mathrm{cc}} / t_{\mathrm{rh}_{\mathrm{i}}} \sim 3.5\left(3.83 \cdot 10^{7} \mathrm{yr}\right)$ with the units used, corresponding to an initial half-mass 
a)

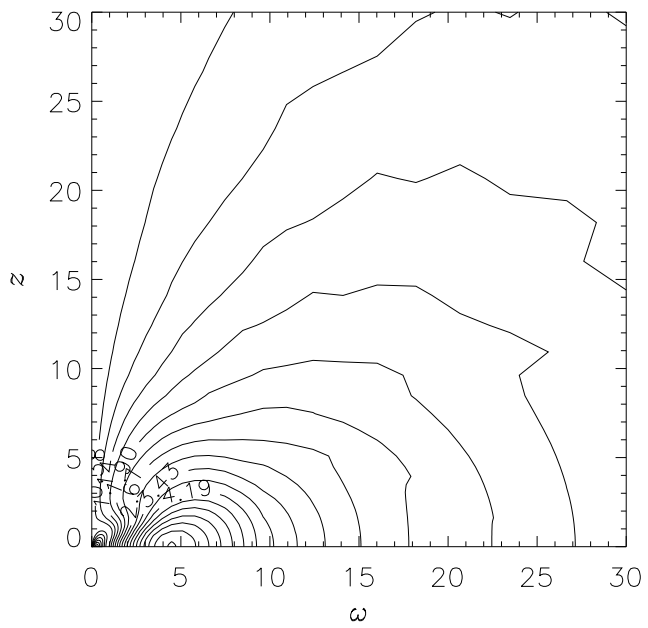

c)

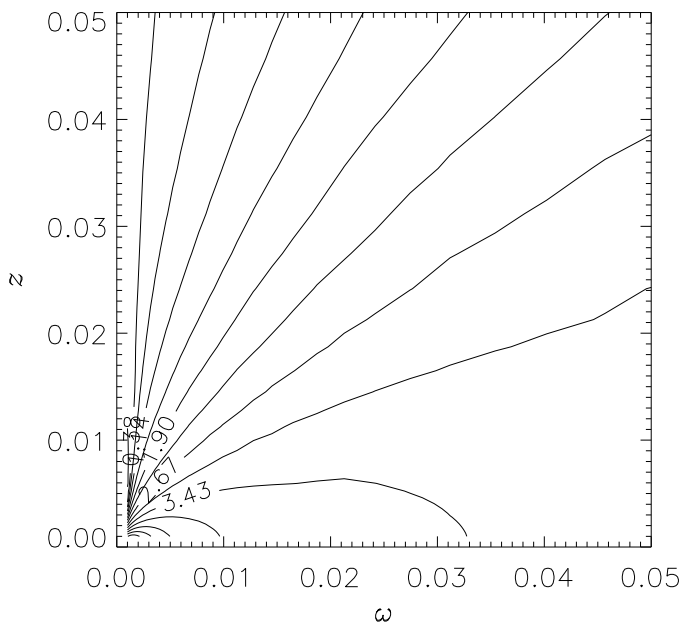

b)

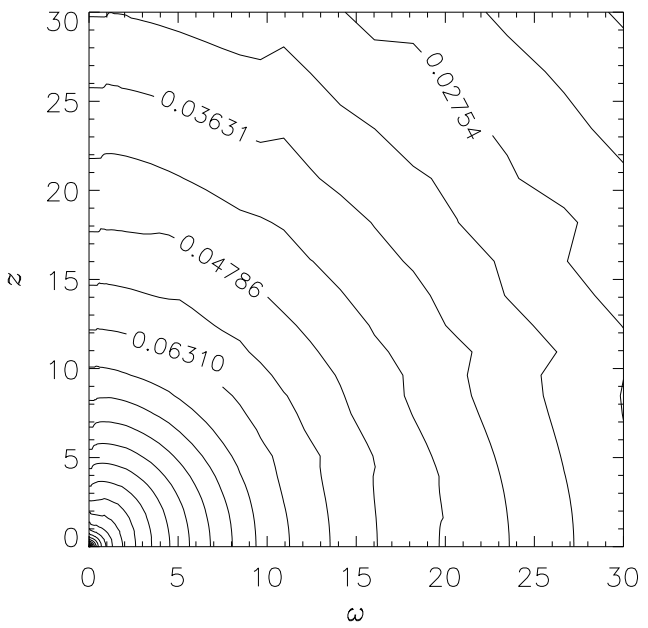

d)

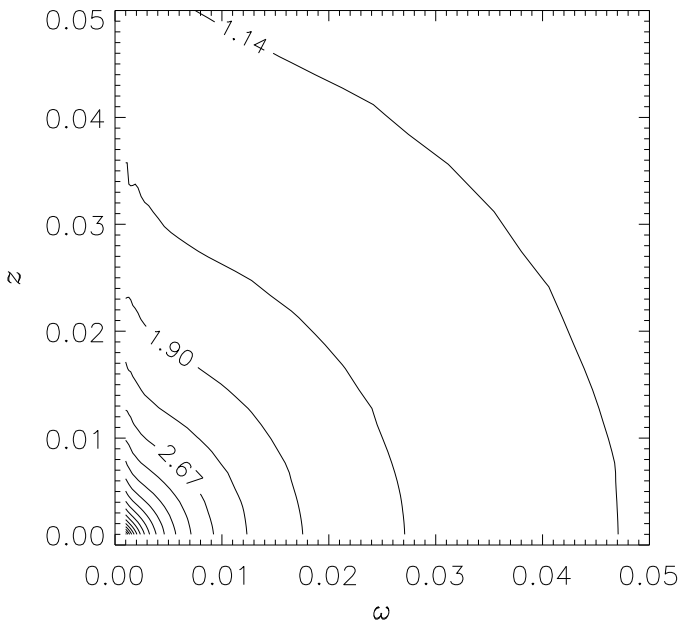

Figure 4.38: Contour map of the rotational velocity and the total velocity dispersion (panels a and $\mathrm{b}$ ) in the meridional plane, as can be obtained by observations, for a collapsed model B4 $\left(6.0,0.9,5 \cdot 10^{-6}\right)$. The bottom panels (c and $\left.\mathrm{d}\right)$ show a zoom of the central parts of the cluster. Observe the increase of central rotation in torus-like contours of $v_{\text {rot }}$ and the second maxima formed in central regions close to the BH. $\sigma_{t}$ shows higher contour levels in the center. Velocities are given in $\mathrm{km} / \mathrm{sec}$ and radii in parsec. The BH mass fraction over the initial cluster mass is $M_{\mathrm{bh}} / M_{\mathrm{cl}} \sim 0.01$ at $t_{\mathrm{cc}} / t_{\mathrm{rh}} \sim 3.5$. 
relaxation time of $t_{\mathrm{rh}_{\mathrm{i}}}=1.09 \cdot 10^{7} \mathrm{yr}$ ). The maxima of central rotation is $4.9 \mathrm{~km} / \mathrm{sec}$ and the respective maxima in velocity dispersion is $7.7 \mathrm{~km} / \mathrm{sec}$.

Young globular clusters are usually more flattened than older ones. This was observed in the Magellanic clouds (Kontizas et al., 1990; Elson et al., 1987,1989) and in LMC, MW and M31 (Han \& Ryden, 1994); and theoretically studied (Frenk \& Fall, 1982). While observations, especially using the utmost accuracy for single star measurements in globular clusters with the Hubble Space Telescope (e.g. Anderson \& King, 2003; Rich et al. 2005) provide a marvelous detail of morphological and sometimes surprising kinematical information, appropriate modelling of elementary features of flattening and rotation (and its dynamical implication for rotational and dispersion velocities) are necessary. The use of detailed evolutionary models should make possible to compare them easily with observations. The basic concept is that one can go in it using simple global observational data (dynamical age, flattening and/or concentration of a cluster), and then pick from a small number of theoretical evolved models to compare with. Following back the structural evolution of the FP-models investigated (e.g. Fiestas \& Spurzem 2005). As the current state of GCs regarding ellipticity shows a high sphericity of this objects, a significant amount of flattening of Globular Clusters is to be expected for the time of their formation and shortly afterwards. 


\section{Chapter 5}

\section{Conclusions}

The variety of environments in which GCs form and evolve, make them unique laboratories for fundamental dynamical processes. Observational studies of GCs have been considerably improved in the last years, leading to a better understanding of these objects, but at the same time, opening new questions due to the, in the past unimpressive, complexity of this stellar systems. This is the reason, why theoretical models are of importance to elucidate the origin of the observed phenomena, and to be able to explain cluster formation and evolution, and predict possible evolutionary scenarios, which can be confirmed by observations in the near future.

The present study contains theoretically formulated evolutionary models of globular star clusters, extending the model complexity through the implementation of cluster rotation and $\mathrm{BH}$ star accretion. On one side flattening supported by rotation is a well known phenomena in GCs and there still not exist a wide set of models, which can explain all the evolutionary implications in the evolution of rotating systems. On the other side, observational evidences of the existence of central dark objects in some GCs, with and without rotation, motivates the implementation of this constraint in the long term evolution. Intermediatemass black holes (IMBHs) may have formed in young clusters (like in the GCS of the LMC).

This work accomplishes for the first time, a complete set of self-consistent evolutionary models of GCs with an embedded $\mathrm{BH}$ and system rotation. It takes advantage of an statistical (Fokker-Planck) method, which allows the computation of a large number of stellar components, and follows the system development in large times scales by a cheap computational time.

The main theoretical conclusion of this work, is related first to the way how the presence of a central dark object supports the evolution of central rotation in self-gravitating relaxed stellar systems; and second, at the same time, how system rotation leads to BH growing, through stellar accretion. Evolution in a galaxy tidal field, which causes evaporation of stars across the tidal boundary, was included in a simple way (circular orbit at constant density within the tidal radius). It is of importance due to the common evolution of GCs hosted by galaxies. The existent models of rotating self-gravitating systems without $\mathrm{BH}$ and spherically symmetric BH models without rotation, which has been widely theoretically developed and verified through observational analyses, are in agreement with the 
results presented here and confirm final states of equilibrium in this kind of objects.

The Fokker-Planck approximation used in this work allows a detailed analysis of energy and angular momentum transport in a self-consistent way. The system evolves in relaxation time scales, before it dissolves in the galaxy tidal field. Evolution is driven by relaxation due to small angle scatterings $(\Delta v / v \ll 1)$, implemented by the use of velocity diffusion coefficients up to second order approximation. There is no correlation between collisions (like in three body collisions), which could, nevertheless, be important for energy generation in the core that can reverse the collapse. In fact the $\mathrm{BH}$ potential represents an energy source in the system.

The loss-cone problem was fully treated as driven by angular momentum diffusion. A star was probably disrupted if its z-component of angular momentum is less than a minimum angular momentum $J_{\mathrm{z}}^{\min }$ and if its time scale of diffusion does not allow it to escape out of the loss-cone. A 'seed' initial $\mathrm{BH}$ with a small mass in comparison to $M_{\mathrm{cl}_{\mathrm{i}}}$ grows slowly, through accretion of mainly low- $J_{z}$ stars, leading to a growing central potential cusp. Stellar distribution around the central BH was represented by a single-particle system, initially axisymmetric in real space and anisotropic in velocity space. No stellar spectrum was included in the model.

In the present study, non-ergodicity on the hyper-surface (given by $E$ and $J_{\mathrm{z}}$ ) is neglected due to any third integral $I_{3}$. The main disadvantage of this assumption lays in the description of halo orbits, where angular momentum is also higher. Furthermore, the potential close to the $\mathrm{BH}$ is spherically symmetric $(\sim 1 / r)$, and $I_{3}$ could be fairly approximated by $J^{2}$, specially at later evolutionary times, when the BH potential cusp dominates.

Results can be summarized as follows:

- Evolutionary states of initially rotating globular clusters, with embedded star accreting black holes were followed in, long-term, relaxation time scales, in order to evaluate the importance of the onset of gravo-gyro vs. gravo-thermal instabilities. Following results are obtained:

- Gravo-gyro effects are coupled to gravo-thermal instabilities and drive core collapse. From a comparison to non-BH models, a further acceleration of collapse occurs due to the presence of the BH. It acts, as an energy source, through which energetic stellar orbits are formed, which easily go into the loss-cone or are lost at the galaxy tidal boundary.

Models $W_{0}=3.0$ with larger cores, and generally lower ellipticities, experiment a more efficient core heating and angular momentum transport, reversing collapse always earlier and at lower density maxima, the higher the rotation. A higher rotation accelerates also cluster mass loss through the galactic tidal boundary and supports BH star accretion. The highest acceleration of collapse is observed in this models. $W_{0}=6.0$ models experience a similar acceleration of collapse, but they have the longest core collapse times, while the highest concentrated models $\left(W_{0}=9.0\right)$ are unstable to gravothermal instabilities leading to the shortest collapse times.

- BHs can reverse collapse, leading to post-collapse phase. Stars in the core are heated via the consumption of stars in bound, high energetic orbits in the cusp. 
The outward flux of energy is achieved by small angle, two-body encounters in the cusp, by which some stars lose energy and move closer to the BH being eventually consumed, while the neighboring stars with which they interact gain energy and move outward from the cusp into the ambient core. At the same time, gravogyro effects carry out angular momentum from the core, increasing in this way the population of stars in low- $J_{\mathrm{z}}$ orbits, and thus the BH mass accretion rate. Initially, mainly unbounded circular tidal orbits in the halo are disrupted at the tidal boundary.

- The cluster becomes a spherical shape, while rotation is lost, faster in comparison with models without $\mathrm{BH}$. As BH accretion of stars on orbits of low $z$-component of angular momentum sets off, an ordered motion of high-angular momentum bounded orbits around the central $\mathrm{BH}$, is built, supporting central rotation. Relaxation effects in the halo lead to interaction of halo stars mainly with stars in the core rather than with other halo stars, owing to the low halo density. Transfer of energy and angular momentum to these orbits, reduces their eccentricities or leads to their loss at the tidal boundary or accretion by the BH (if their energies are high and their angular momentum small enough, inside the loss-cone). As the system loses its angular momentum due to gravogyro instabilities, more stars are transported from the core to the outer regions, depleting the population of core orbits, and consequently, core rotation.

- Equilibrium states of systems with BH were obtained. Density and velocity dispersion profiles do not show apparently variations from the well known spherical systems, although the cluster is initially flattened, while mass loss is enhanced by the presence of the BH. In detail,

- Final steady state solutions are applicable to rotating GCs. All models approximate the $\lambda=-1.75$ density cusp and the $\gamma=-0.5$ velocity dispersion cusp inside the $\mathrm{BH}$ influence radius $r_{\mathrm{a}}$, corresponding to Keplerian bounded orbits, independent of initial concentration and rotation. Moreover, at later times, the system achieves a concentration of stars in the equatorial plane, very close to the $\mathrm{BH}$, than in the zenithal direction along the axis of rotation.

- The final $M_{\mathrm{BH}}$ stalls at $\sim 0.01 M_{\mathrm{cl}}$ at collapse time, and remains nearly constant in the post-collapse phase, while $\mathrm{BH}$ mass accretion rate $\left(d M_{\mathrm{BH}} / d t\right)$ reaches a maximum at collapse time, due to the higher density of orbits in the core, and falls afterwards. Cluster mass $\left(M_{\mathrm{cl}}\right)$ loss in the tidal field of the parent galaxy is very strong during the re-expansion of the core. The cluster mass reaches very rapid values at least one order of magnitude smaller than its hosted $\mathrm{BH}$.

The BH-mass limit for a typical cluster of $5 \cdot 10^{6} M_{\odot}$, varies between $1.9 \cdot 10^{3} M_{\odot}$ and $9.5 \cdot 10^{4} M_{\odot}$ using models of different concentrations and initial rotation. This is a good approximation of the mass of IMBH estimated by theoretical studies and observations. The general behavior exhibits an increasing final $M_{\mathrm{BH}}$ as well as higher $d M_{\mathrm{BH}} / d t$ corresponding to higher initial rotation $\left(\omega_{0}\right)$.

As angular momentum diffusion outwards (gravogyro instability) drives at early times tidal mass loss of circular pro-grade halo orbits, radial anisotropy in the halo increases, as well as the probability of $\mathrm{BH}$ star accretion of low- $J_{\mathrm{z}}$ orbits. The initial positive (radial) halo anisotropy is slowly depleted, while negative 
(tangential) anisotropy forms first inside the $\mathrm{BH}$ influence radius. This process accelerates at collapse time. After collapse, tangential orbits dominate all regions of the system $(A<0)$. Thus, gravogyro driven tidal mass loss supports $\mathrm{BH}$ star accretion and leads to an increasingly $\mathrm{BH}$ mass growth rate during core collapse.

- Observational measurements of rotation can be used together with GC parameters as concentration and ellipticities, to prove the presence of central BHs in stellar clusters. Nevertheless, high core resolution is needed (less than $0.1 \mathrm{pc}$ ). Young clusters are natural targets to look for rotation, but the embedded BHs should be smaller at early evolutionary times $\left(M_{\mathrm{BH}} \lesssim 10^{2} M_{\odot}\right)$. Furthermore,

- Rotation is a good descriptor in early evolutionary states.

Central rotational velocity grows slowly in the initial evolutionary states, and develops during the evolution up to collapse time, when the maxima of rotation $\left(V_{\text {rot }}^{\max }\right)$ at the center dominates over the original maxima at around the half-mass radius. In the outer parts, rotation is continuously depleted, due to angular momentum loss. Later, at the end of evolution, i.e. close to cluster dissolution, the cluster has lost nearly all its rotational energy.

Rotation, like stellar density, grows preferably in $\varpi$-direction (in the equatorial plane), what confirms its influence in the redistribution of stars in the cluster, building ordered motion of bounded stars around the BH.

Central rotational velocity grows in retrograde direction with respect to the halo (counter-rotation), under the influence of the $\mathrm{BH}$ potential cusp, in a similar way as pro-grade stellar orbits in the halo, are initially preferably lost through the outer boundary in the galactic tidal field. Close to the $\mathrm{BH}$, where angular momentum is initially small (for tightly bounded stars), orbits are preferably ordered in retrograde direction, due to higher diffusion of negative angular momentum (pro-grade) orbits outwards (gravogyro effect). As halo stars interact with core stars more than with other halo stars, tidal galaxy effects influence also the redistribution of orbits in the core.

- $W_{0}=6.0$ models seem to maintain rotation increasing at collapse and short time after collapse, leading to a higher rotating collapsed core in comparison with other models. They show also, in general, higher ellipticities. They are able to maintain an efficient angular momentum diffusion, increasing core rotation, and at the same time are concentrate enough to avoid an excessive mass loss. Both effects support the accretion of stars in low- $J_{z}$ orbits and an ordered motion around the $\mathrm{BH}$ at the cluster center. The amount of rotation present in the core at later times, i.e. at collapse and afterwards, depends on the efficiency of angular momentum diffusion due to relaxation and $\mathrm{BH}$ star accretion, specially before most of angular momentum is driven away.

$W_{0}=3.0$ models can not develop efficiently core rotation, as angular momentum transport is highly efficient and mass is rapidly lost. The shortest collapse times in $W_{0}=9.0$ models do not allow the development of core rotation due to an inefficient angular momentum transport outwards (gravogyro effect).

- Random vs. ordered motion during the evolution of BH-models, develops a growing $V_{\text {rot }} / \sigma$ already at early times and a further increasing up to collapse time. 
This behavior always depends on (a high) initial system rotation and an efficient angular momentum diffusion and $\mathrm{BH}$ star accretion, which are able to lead to an increasing in central rotational velocity before angular momentum is consumed. $W_{0}=6.0$ models show a stable evolution of $V_{\text {rot }} / \sigma$, with an increase of central rotation and decrease of dispersion up to collapse, and a slightly increase of both after collapse time. $V_{\text {rot }} / \sigma \lesssim 1$ at the time of maximum rotation $\left(\sim t_{\mathrm{cc}}\right)$. This result is comparable with the central $V_{\text {rot }} / \sigma$ profile of the galactic GC M15 (Gebhardt et al. 2000; Gerssen et al. 2002, 2003). These authors reported, from observational studies, a maximum of $V_{\text {rot }} / \sigma \sim 1$ ).

Like in non-BH models, angular momentum loss accelerates in the post-collapse phase, leading to a central depletion of the $V_{\text {rot }} / \sigma$ profile. Rotation does not survive later in the post-collapse phase, leading to a final nearly spherical reexpanding cluster, embedding a central IMBH with a mass of $\sim 1 \% M_{\mathrm{cl}}$. The final destiny of the cluster is its dissolution through galaxy tidal forces, leaving an IMBH behind, orbiting its parent galaxy.

The relative simplified assumptions of the present simulations listed in Sect. (3.1.1) make clear that some constraints in the evolution of rotating clusters are still missing, like a mass spectrum or stellar evolution, as well as a more realistic criterium for galactic tidal mass loss (shocks). This are targets for further development of the code. Comparison to N-Body models are aimed to complement this calculations, using the highest particle number permitted at the time $\left(N \sim 10^{6}\right)$. Regarding the stellar spectrum, the distribution of high mass stars is expected to drop the dispersion in the center (as reported by Kim et al., 2004), leading maybe to a higher or at least more stable $V_{\text {rot }} / \sigma$ in this region. Nevertheless, the agreement of this profile and estimation of $\mathrm{BH}$ masses with observations and the consistence of the general evolution of cluster structure in systems including embedded BHs, with current theoretical studies without rotation, makes clear that rotation constitutes an important constraint, which needs to be taken into account for the understanding of the formation and evolution of GCs, specially when rotation is high enough, at early times of evolution (e.g. in the young clusters of the LMC).

As a general conclusion, the amount of rotation present in the system during its dynamical evolution is a result of the interplay between diffusion of angular momentum (gravogyro instability) and the redistribution of high energy orbits close to the $\mathrm{BH}$ (loss-cone effect). The central $\mathrm{BH}$ affords the formation of an angular momentum source near the center, which supports gravogyro instabilities leading to core collapse. In a further speculation, as the central BH is able to 'consume' angular momentum of the system it may become itself an angular momentum source, which would be able to rotate (Kerr Black Hole), permitting also a more efficient angular momentum transport outwards, through interaction with core stars, driven by relaxation. A binary black hole (BBH) system, could, in a similar way, lead to a more efficient support in the development of central rotation, modifying substantially the final shape of the cluster.

The present work gives theoretical constraints in the evolution of globular clusters under the assumption of the formation and evolution of a central dark object, and formulates predictions about the later evolutionary states of such stellar systems. The models presented, 
are able to reproduce $2 \mathrm{D}$ distributions (in the meridional plane) of density, cluster- and $\mathrm{BH}$ potential, velocity dispersions, rotational velocity, anisotropy, dynamical ellipticity, among other parameters, at any time of evolution and deep in the stellar cusp surrounding the central BH. It makes possible to study the evolution of kinematical and structural parameters (radii, time scales, concentration, ellipticity), which complement and test observational measurements. As mentioned in Sect. (1.4) observational studies of suspected globular clusters with BHs include galactic and extragalactic cluster types, which can be compared to evolutionary models, to elucidate theoretical predictions and understand the common evolution of star clusters and galaxies. With this aim, a detailed set of model data, covering a wide range of rotation rates and initial concentrations of rotating GCs, with and without $\mathrm{BH}$, has been created, to enable observers to use them for comparisons with their data *

${ }^{*}$ The cluster database can be found on the web, at http://www.ari.uni-heidelberg.de/clusterdata/ 


\section{Bibliography}





\section{Bibliography}

[1] Aarseth S. J., 1999, Publ. Astron. Soc. Pac., 111, 1333

[2] Aarseth S.J. \& Heggie, D.C., 1993, ASP Conf. Ser, 48, 701

[3] Ajhar et al. 1996, Astron. J., 111, 1110

[4] Akiyama A. \& Sugimoto D., 1989, Publ. Astron. Soc. Japan, 1, 991

[5] Alcaino et al., 1990, Astrophys. J. Suppl., 72, 693

[6] Amaro-Seoane P. \& Spurzem R., 2001, Mon. Not. Royal Astron. Soc., 327, 995

[7] Amaro-Seoane P., Freitag M. \& Spurzem R., 2004, Mon. Not. Royal Astron. Soc., 352, 655

[8] Anderson J. \& King, I.R. 2003, Astron. J., 126, 772

[9] Ashman K. M. \& Zepf S. E., 1992, Astrophys. J., 384, 50

[10] Ashman K. M. \& Zepf S. E., 1998, Obs, 118, 387

[11] Bahcall J. N. \& Wolf R. A., 1977, Astrophys. J., 216, 883

[12] Barmby P. \& Huchra, J. P. 2001, Astron. J., 122, 2458

[13] Barmby P. et al. 2002, Astron. J., 123, 1937

[14] Baumgardt H., 2001, Mon. Not. Royal Astron. Soc., 325, 1323

[15] Baumgardt et al. 2003, Astrophys. J., 582, 21

[16] Baumgardt H. \& Makino J., 2003, Mon. Not. Royal Astron. Soc., 340, 227

[17] Baumgardt H., Heggie D. C., Hut P. \& Makino J., 2003, Mon. Not. Royal Astron. Soc., 341,247

[18] Baumgardt H.,Makino J. \& Ebisuzaki T., 2004, Astrophys. J., 613, 1143

[19] Baumgardt H., Makino J. \& Hut P., 2005, Astrophys. J., 620, 238

[20] Berczik P., Merritt D. \& Spurzem R., 2005, Astrophys. J., in press, arXiv:astro$\mathrm{ph} / 0507260$

[21] Bellazzini 1998, New Astron., 3, 219 
[22] Bettwieser E. \& Sugimoto, D., 1984, Mon. Not. Royal Astron. Soc., 208, 493

[23] Binney J.J. \& Tremaine S.D. 1987. Galactic Dynamics, Princeton: PUP (三 BT)

[24] Boily C. M. \& Kroupa P., 2003a, Mon. Not. Royal Astron. Soc., 338, 665

[25] Boily C. M. \& Kroupa P., 2003b, Mon. Not. Royal Astron. Soc., 338, 673

[26] Brocato et al., 1998, Astron. \& Astroph., 335, 929

[27] Brown T. et al. 2003, Astrophys. J., 592, 17

[28] Brown T. et al. 2004a, Astron. J., 127, 2738

[29] Brown T. et al. 2004b, Astrophys. J., 613, L125

[30] Cen R., 2001, Astrophys. J., 560, 592

[31] Chang J.S. \& Cooper G., 1970, J. Comp.Phys., 6,1

[32] Chernoff D. \& Djorgovski S., 1989, Astrophys. J., 339, 904

[33] Caputo et al., 1984, Astron. \& Astroph., 138, 457

[34] Clarke C. J., \& Pringle J. E. 1992, Mon. Not. Royal Astron. Soc., 255, 423

[35] Cohen J. G., 2000, Astron. J., 119, 162

[36] Cohn H., 1980, Astrophys. J., 242, 765

[37] Colpi et al. 2003, Astrophys. J., 599, 1260

[38] Coté P., 1999, Astron. J., 118, 406

[39] de Grijs R. et al., 2002a, Mon. Not. Royal Astron. Soc., 331, 228

[40] de Grijs R. et al., 2002b, Mon. Not. Royal Astron. Soc., 337, 597

[41] Dehnen W., Odenkirchen M., Grebel E. K. \& Rix H.-W., 2004, Astron. J., 127, 2753

[42] Deiters S. et al., 2001, Dynamics of Star Clusters and the Milky Way, ASP Conference Series, Vol. 228.

[43] Djorgovski 1995, Astrophys. J., 438, L29

[44] Djorgovski 1996, IAU Symp.174

[45] Djorgovski et al. 1997, Astrophys. J., 474, 19

[46] Djorgovski S. \& Santiago, B. X., 1992, Astrophys. J., 391, 85

[47] Djorgovski \& Meylan 1994, Astron. J., 108, 1292

[48] Dressler A. \& Richstone D. O., 1988, Astrophys. J., 324, 701

[49] Drukier G. A., Fahlman G. \& Richer H, 1992, Astrophys. J., 386, 106

[50] Dubath \& Grillmair 1997, Astron. \& Astroph., 321, 379 
[51] Ebisuzaki T. et al., 2001, Astrophys. J., 562, 1

[52] Einsel C. \& Spurzem R., 1999, Mon. Not. Royal Astron. Soc., 302, 81

[53] Einsel C., 1998, In Duschl W. \& Einsel Ch., Proceedings Series Dynamics of Galaxies and Galactic Nuclei, Vol. 02, pg.31

[54] Elmegreen B. G. \& Efremov Y. N., 1997, Astrophys. J., 480, 235

[55] Elson, R.A.W et al.,1987,Astrophys. J., 323,54

[56] Elson R.A.W., Freeman, K.C. \& Lauer, T.R. 1989, Astrophys. J., 347,69

[57] Fall S.M. \& Frenk, C.S. 1985. In IAU Symposium 113, J. Goodman \& P. Hut (eds), Dordrecht: Kluwer, p. 285

[58] Fall S. M. \& Rees M. J., 1985, Astrophys. J., 298, 18

[59] Ferguson A., et al. 2002, Astron. J., 124, 1452

[60] Ferrarese L., et al., 2001, Astrophys. J. Lett., 555, 79

[61] Ferrarese L. \& Merritt D., 2000, Astrophys. J., 539, 9

[62] Ferraro et al. 2003, Astrophys. J., 595, 179

[63] Forbes D. et al., 2001, Astrophys. J., 556, 83

[64] Frank J. \& Rees M., 1976, Mon. Not. Royal Astron. Soc., 176, 633

[65] Fregeau J. M., Gürkan M. A., Joshi K. J., Rasio F. A., 2003, Astrophys. J., 593, 772

[66] Freitag M. \& Benz W., 2002, Astron. \& Astroph., 394, 345

[67] Frenk C.S. \& Fall, S.M., 1982, Mon. Not. Royal Astron. Soc., 199, 565

[68] Frenk C.S. \& White, S.D.M., 1982, Mon. Not. Royal Astron. Soc., 198, 173

[69] Fusi Pecci, F. et al. 1996, Astron. J., 112, 1461

[70] Garcia \& Mermilliod, 2001, Astron. \& Astroph., 368,122

[71] Gebhardt K. et al., 1994, Astron. J.107, 2067

[72] Gebhardt K. \& Fischer, P., 1995, Astron. J.109, 209

[73] Gebhardt K. et al., 2000, Astron. J.119, 1268

[74] Gebhardt K. et al., 2002, Astrophys. J., 578, 41

[75] Gebhardt K. \& Kissler-Patig M., 1999, Astron. J., 118, 1526

[76] Ghez A. M. et al., 2003, Astrophys. J. Lett., 586, 127

[77] Gerssen J. et al., 2002, Astron. J., 124,3270

[78] Gerssen J. et al., 2003, Astron. J., 125, 376 
[79] Geyer E. H., Nelles B. \& Hopp U., 1983, Astron. \& Astroph., 125, 359

[80] Gezari S. et al., 2003, Astrophys. J., 592, 42

[81] Giersz M., 1996, IAUS, 174, 101

[82] Giersz M., 1998, Mon. Not. Royal Astron. Soc., 298, 1239

[83] Giersz M., Heggie D. C., 1994a, Mon. Not. Royal Astron. Soc., 270, 298

[84] Giersz M., Heggie D. C., 1994b, Mon. Not. Royal Astron. Soc., 268, 257

[85] Giersz M. \& Heggie, D.C., 1997, Mon. Not. Royal Astron. Soc.,286, 709

[86] Giersz M. \& Spurzem, R., 1994, Mon. Not. Royal Astron. Soc., 269, 241

[87] Giersz M., \& Spurzem, R. 2000, Mon. Not. Royal Astron. Soc., 317, 581

[88] Giersz M. \& Spurzem R., 2003, Mon. Not. Royal Astron. Soc.343, 781

[89] Gnedin O. Y. \& Ostriker J. P. 1997, Astrophys. J., 474, 223

[90] Gnedin O. Y., Lee H. M., Ostriker J. P., 1999, Astrophys. J., 522, 935

[91] Gnedin O. Y., Hernquist L., Ostriker J. P., 1999, Astrophys. J., 514, 109

[92] Goodman, J. 1983. PhD Thesis, Princeton University

[93] Goodwin S. P. 1997, Mon. Not. Royal Astron. Soc., 284, 785

[94] Gouliermis D., Keller S., Kontizas M., Kontizas E. \& Bellas-Velidis I. 2004, Astron. \& Astroph., 416, 137

[95] ratton R. G. et al., 1997, Astrophys. J., 491, 749

[96] Grillmair Carl J. et al., 1999, Astron. J., 117, 167

[97] Gualandris A., Portegies Zwart S., \& Eggleton P. P. 2004, Mon. Not. Royal Astron. Soc., 350,615

[98] Grundahl F. et al., 2002, Astron. \& Astroph., 395, 481

[99] Gürkan M., Freitag M. \& Rasio F., 2004, Astrophys. J.,604,632

[100] Hachisu I., 1979, Publ. Astron. Soc. Japan, 31,523

[101] Hachisu I., 1982, Publ. Astron. Soc. Japan, 34,313

[102] Han C. \& Ryden B.S., 1994,Astrophys. J., 433,80

[103] Hanes D. A., 1977, Mem.R.A.S., 84, 45

[104] Harris W. E. \& Racine R., 1979, Ann. Rev. Astron. Astroph., 17, 241

[105] Harris W. E. \& Pudritz R. E., 1994, Astrophys. J., 429, 177

[106] Harris H. C., et al, 1985, Astron. J., 90, 2495 
[107] Harris W.E., 1996, Astron. J., 112, 1487

[108] Harris W. E., 2001, stcl.conf, 223

[109] Heggie D.C., 1984, Mon. Not. Royal Astron. Soc., 206, 179

[110] Heggie D.C., 2003, IAUS, 208, 103

[111] Heggie, D.C. et al., 1998. Highlights of Astronomy, Vol. 11. Andersen, J. (ed), Dordrecht: Kluwer (astro-ph/9711191)

[112] Hemsendorf M.; Sigurdsson S. \& Spurzem R., 2002, Astrophys. J., 581, 1256

[113] Henyey G. R., Wilets L.,Böhm K.H., Le Levier R., Leevee R.D., 1959,Astrophys. J., 129,628

[114] Hills J. G., 1975, Nat, 254, 295

[115] Hills J. G., 1980, Astrophys. J., 235, 986

[116] Holland S., et al. 1997, Astron. J., 114, 1488

[117] Hopman C., Portegies Zwart S. \& Alexander, T. 2004, Astrophys. J., 604,101

[118] Hurley J. R., Tout C. A., Aarseth S. J., Pols O. R., 2001, Mon. Not. Royal Astron. Soc., 323,630

[119] Hurley J. R., Pols, O. R., Aarseth, S. J., \& Tout, C. A. 2005, ArXiv Astrophysics e-prints, arXiv:astro-ph/0507239

[120] Hut P., 1985, IAU Symp., 113, 231

[121] Ibata R. A. et al.,1999, Astrophys. J. Suppl., 120, 265

[122] Ivanova N., Belczynski K., Fregeau J. M., \& Rasio F. A. 2005, Mon. Not. Royal Astron. Soc., 358, 572

[123] Jablonka P. et al., 2000, Astron. \& Astroph., 359, 131

[124] Jarvis B. J. \& Dubath P., 1988. Astron. \& Astroph., 201, 33

[125] Karovska et al., 2002, Astrophys. J., 577, 114

[126] Kawakatu, N. \& Umemura, M., 2004, Astrophys. J., 601, 21

[127] Kerber L.O., \& Santiago, B.X., 2005, Astron. \& Astroph., 435, 77

[128] Kim E., Einsel C., Lee H.M., Spurzem, R., Lee M.G., 2002, Mon. Not. Royal Astron. Soc., 334,310

[129] Kim E., Lee H.M. \& Spurzem R., 2004, Mon. Not. Royal Astron. Soc., 351,220

[130] King I. R., 1966, Astron. J., 71, 64

[131] King A. R., Davies M. B., Ward M. J., Fabbiano G., \& Elvis M. 2001, Astrophys. J., 552,109 
[132] Kissler-Patig M. et al., 1997 Astron. \& Astroph., 319, 470

[133] Koch A., Grebel E. K., Odenkirchen M., Martínez-Delgado D., Caldwell J. A. R., 2004, Astron. J., 128, 2274

[134] Kontizas E. et al., 1990, Astron. J., 100, 425

[135] Kormendy J. 1985, Astrophys. J., 295, 73

[136] Kormendy J., astro-ph/0306353.

[137] Kormendy J. \& Richstone D., 1995, Ann. Rev. Astron. Astroph., 33, 581

[138] Krauss L. M. \& Chaboyer B., 2003, Sci, 299, 65

[139] Kroupa P., 1995a, Mon. Not. Royal Astron. Soc., 277, 1522

[140] Kroupa P., 1995b, Mon. Not. Royal Astron. Soc., 277, 1507

[141] Kroupa P., 1995c, Mon. Not. Royal Astron. Soc., 277, 1491

[142] Kroupa P. 1998, Mon. Not. Royal Astron. Soc., 298, 231

[143] Kroupa P. 2000, New Astron., 4, 615

[144] Kroupa P., 2002, Science, 295, 82

[145] Kroupa P. 2004, New Astronomy Review, 48, 47

[146] Kroupa P. 2005, ESA SP-576: The Three-Dimensional Universe with Gaia, 629

[147] Kroupa P., Petr M. G., \& McCaughrean M. J. 1999, New Astron., 4, 495

[148] Kroupa P., Aarseth S., \& Hurley J. 2001, Mon. Not. Royal Astron. Soc., 321, 699

[149] Kroupa P. \& Burkert A., 2001, Astrophys. J., 555, 945

[150] Kroupa P. \& Bouvier J., 2003a, Mon. Not. Royal Astron. Soc., 346, 343

[151] Kroupa P. \& Bouvier J., 2003b, Mon. Not. Royal Astron. Soc., 346, 369

[152] Kroupa P., Aarseth S., Hurley J., 2001, Mon. Not. Royal Astron. Soc., 321, 699

[153] Kroupa P., Bouvier J., Duchêne G., Moraux E., 2003, Mon. Not. Royal Astron. Soc., 346,354

[154] Larsen et al. 2001, Astron. J., 121, 2974

[155] Larsen et al. 2002, Astron. J., 124, 2615

[156] Lagoute C. \& Longaretti P. Y., 1996, Astron. \& Astroph., 308, 441

[157] Lee M. H. \& Goodman J.,1989, Astrophys. J., 343, 594

[158] Lightman A. P. \& Shapiro S. L. 1977, ApJ, 211, 244

[159] Longaretti P. Y. \& Lagoute C., 1997, Astron. \& Astroph., 319, 839 
[160] Louis P. \& Spurzem R., 1991, Mon. Not. Royal Astron. Soc., 251, 408

[161] Lupton R.H., Gunn J.E. \& Griffin R.F., 1987, Astron. J., 93, 1114

[162] Lynden-Bell D. \& Eggleton P. P., 1980, Mon. Not. Royal Astron. Soc., 191, 483

[163] Mackey A. D., \& Gilmore G. F. 2003, Mon. Not. Royal Astron. Soc., 338, 85

[164] Marchant A. B. \& Shapiro S. L., 1980, Astrophys. J., 239, 685

[165] Maschberger T., Kroupa P. 2005, in prep.

[166] Massey P. 2003, Ann. Rev. Astron. Astroph., 41, 15

[167] McNamara et al. 2003, Astrophys. J., 595, 187

[168] McClintock et al., 2003, Astrophys. J., 593, 435

[169] McLaughlin 2000, Astrophys. J., 539, 618

[170] McLaughlin D. E. etal., 2003, In: New Horizons in Globular Cluster Astronomy, ASP Conference Proceedings, Vol.296, 101

[171] Merritt D. et al., 1997,Astron. J., 114, 1074

[172] Merritt D. \& Poon M. Y., 2004, Astrophys. J., 606, 774

[173] Meylan G. \& Heggie D. C., 1997, A\&ARv, 8, 1

[174] Meylan G. \& Mayor, M., 1986, Astron. \& Astroph., 166, 122

[175] Mikkola S., Aarseth S. J., 1990, CeMDA, 47, 375

[176] Mikkola S., Aarseth S. J., 1993, CeMDA, 57, 439

[177] Mikkola S., Aarseth S. J.,1996, New Astron., 3, 309

[178] Mikkola S., Aarseth S. J.,1998, CeMDA, 64, 197

[179] Miller J. M. et al., 2003, Astrophys. J., 585, 37

[180] Miller M. C., \& Colbert, E. J. M. 2004, Int. J. Mod. Phys. D, 13, 1

[181] Miller M. \& Hamilton, D., 2002, Mon. Not. Royal Astron. Soc., 330, 232

[182] Milosavljevic \& Merritt, 2001, 563, 34

[183] Milosavljevic \& Merritt, 2003, Astrophys. J., 596, 860

[184] Minniti D. et al. 1995, Mon. Not. Royal Astron. Soc., 277, 1293

[185] Mouri H. \& Taniguchi, Y. 2002, Astrophys. J., 566, 17

[186] Murphy B., Cohn H. \& Durisen R., 1991, Astrophys. J., 370, 60

[187] Peebles P. J. E., 1972, Astrophys. J., 178, 371

[188] Pinkney J. et al., 2003, Astrophys. J., 596, 903 
[189] Preto M., Merritt D., Spurzem R., 2004, Astrophys. J., 613, 109

[190] Paresce F. \& de Marchi, G. 2000, Astrophys. J., 534, 870

[191] Piotto G. \& Zoccali M., 1999, Astron. \& Astroph., 345, 485

[192] Piotto G. et al., 1999, Astron. J., 118, 1727

[193] Portegies Zwart S. et al., 1999, Astron. \& Astroph., 348, 117

[194] Portegies Zwart S. \& McMillan S., 2002, Astrophys. J., 576, 899

[195] Portegies Zwart S. et al., 2004, Nature, 428, 724

[196] Preibisch T., Balega Y., Hofmann K.-H., Weigelt G., \& Zinnecker H. 1999, New Astron., 4, 531

[197] uinlan G. D.; Hernquist L. \& Sigurdsson S., 1995, Astrophys. J., 440, 554

[198] Rappaport S., Podsiadlowski Ph., \& Pfahl E. 2005, Mon. Not. Royal Astron. Soc., 356, 401

[199] Rauch K. P., 1995, Mon. Not. Royal Astron. Soc., 275, 628

[200] Rich R. M et al., 1996, Astron. J., 111, 768

[201] Rich R. M et al., 2005, Astron. J., 129, 2670

[202] Rosenbluth M. N., MacDonald W. M. \& Judd D. L., 1957, PhRv, 107, 1

[203] Rubenstein E. P.\& Bailyn C., 1997, Astrophys. J., 474, 701

[204] Sandquist et al., 1996, Astrophys. J., 470, 910

[205] Samus et al., 1995, Astron. \& Astrophys. Suppl., 112, 439

[206] Santiago B. et al. 2001, Astron. \& Astroph., 369, 74

[207] Schödel R. et al., 2003, Astrophys. J., 596, 1015

[208] Schweizer F., Proceedings of the Eighth Santa Cruz Summer Workshop in Astronomy and Astrophysics 1986, Springer-Verlag, 1987, p.18-25.

[209] Shara M. et al., 1998, Astrophys. J., 508, 570

[210] Searle \& Sinn, 1978, Astrophys. J., 225, 357

[211] Spitzer L. Jr 1987. Dynamical Evolution of Globular Clusters

[212] Spitzer L. \& Shapiro S. L., 1972, Astrophys. J., 173, 529

[213] Spurzem R., 1996, IAUS, 174, 111

[214] Spurzem R., 1999, Jl. Comp. Appl. Maths., 109, 407

[215] Spurzem R. \& Takahashi K., 1995, Mon. Not. Royal Astron. Soc., 272, 772 
[216] Spurzem R. \& Aarseth S.J., 1996, Mon. Not. Royal Astron. Soc., 282, 19

[217] Spurzem R. \& Giersz M., 1996, Mon. Not. Royal Astron. Soc., 283, 805

[218] Stephens A. W. et al. 2001, Astron. J., 121, 2597

[219] Takahashi K.,1995, Publ. Astron. Soc. Japan, 49, 547

[220] Takahashi K.,1996, Publ. Astron. Soc. Japan, 48, 691

[221] Takahashi K.,1997, Publ. Astron. Soc. Japan, 47,,561

[222] Takahashi K., Lee \& Inagaki, 1997, Mon. Not. Royal Astron. Soc., 292,331

[223] Takahashi K. \& Portegies Zwart S.F., 1998, Astrophys. J., 503, 49

[224] Takahashi K. \& Portegies Zwart S.F., 2000, Astrophys. J., 535, 759

[225] Takahashi K. \& Lee, 2000, Mon. Not. Royal Astron. Soc., 316, 671

[Tanabé et al. 2004] Tanabé et al. 2004, Astrophys. J. Suppl., 155, 401

[226] Thompson I. B. et al., 2001, Astron. J., 121, 3089

[227] Trager S. C., King I. R. \& Djorgovski S., 1995, Astron. J., 109, 218

[228] Tremblay B. \& Merritt D., 1995, Astron. J., 110, 1039

[229] Trudolyubov \& Priedhorsky 2004, Astrophys. J., 616,821

[230] Weidner C., Kroupa P., \& Larsen S. S. 2004, Mon. Not. Royal Astron. Soc., 350, 1503

[231] Van den Bergh 1996, The Observatory, 116, 103

[232] Van Gorkom et al., 1994, Astrophys. J., 423, 101

[233] Van Leeuwen et al., 2000, Astron. \& Astroph., 360, 472

[234] Vesperini E. \& Chernoff D., 1996, Astrophys. J., 458, 178

[235] Vine S. G., \& Bonnell I. A. 2003, Mon. Not. Royal Astron. Soc., 342, 314

[236] White R. E. \& Shawl S. J., 1987, Astrophys. J., 317, 246

[237] Wilkinson M. I., Hurley J. R., Mackey A. D., Gilmore G. F., \& Tout C. A. 2003, Mon. Not. Royal Astron. Soc., 343, 1025

[238] Young P., 1980, Astrophys. J., 242, 1232

[239] Zepf S. E. \& Ashman K. M., 1993, Mon. Not. Royal Astron. Soc., 264, 611

[240] Zepf S. E. et al., 2000, Astron. J., 120, 2928

[241] Zinn R., 1985, Astrophys. J., 293, 424 

Appendix 



\section{Derivation of the flux coefficients}

The approximation of Rosenbluth, MacDonald \& Judd (1957) is carried on for the determination of flux coefficients, involving covariant derivatives of tensorial objects. As cylindrical coordinates are used $(\rho, z, \varphi)$, the following symmetry is applied to velocity space: $q^{1}=v=\left(v_{\rho}^{2}+v_{z}^{2}+v_{\varphi}^{2}\right)^{1 / 2} ; q^{2}=\psi=\arctan \left(v_{\rho} / v_{z}\right) ; q^{3}=v_{\varphi}$, where $\left(v_{\rho}, v_{z}, v_{\varphi}\right)$ are local Cartesian velocity coordinates. The corresponding metric tensor $\left(a^{\mu \nu}\right)$ reads as

$$
a^{\mu \nu}=\left(\begin{array}{ccc}
1 & 0 & \frac{v_{\varphi}}{v} \\
0 & \frac{1}{\left(v_{\rho}^{2}+v_{z}^{2}\right)} & 0 \\
\frac{v_{\varphi}}{v} & 0 & 1
\end{array}\right)
$$

with the volume element $a:=\operatorname{det}\left(a_{\mu \nu}\right)=1 / \operatorname{det}\left(a^{\mu \nu}\right)=v^{2}$. The tensorial form of the Fokker Planck equation may generally be written as

$$
\frac{1}{\Gamma_{a}} \frac{\partial f_{a}}{\partial_{t}}=-\left(f T_{a}^{\mu}\right)^{\prime} \mu+\frac{1}{2}\left(f S_{a}^{\mu \nu}\right)^{\prime} \mu \nu
$$

where the commas denote covariant derivatives, and the subscript $a$ indicates particle species. The factor $\Gamma_{a}=4 \pi G m_{a}^{2} \ln \Delta$ contains the Coulomb logarithm, The diffusion coefficients of Cartesian coordinate systems may then be expressed as tensorial objects

$$
\begin{gathered}
\frac{1}{\Gamma_{a}}<\Delta v^{\mu}>_{a}=T_{a}^{\mu}=a^{\mu \nu}\left(h_{a}\right)^{\prime} \nu \\
\frac{1}{\Gamma_{a}}<\Delta v^{\mu} \Delta v^{\nu}>_{a}=S^{\mu \nu}=a^{\mu \omega} a^{\nu \tau}(g)^{\prime} \omega \tau
\end{gathered}
$$

The functions $h$ and $g$ are the so called Rosenbluth potentials:

$$
\begin{gathered}
h_{a}(\vec{v})=\Sigma_{b} \frac{m_{a}+m_{b}}{m_{b}} \int d \vec{v}_{f}^{\prime} f_{b}\left(\vec{v}_{f}^{\prime}\right) \frac{1}{\left|\vec{v}-\vec{v}_{f}^{\prime}\right|} \\
g(\vec{v})=\Sigma_{b} \int d \vec{v}_{f}^{\prime} f_{b}\left(\vec{v}_{f}^{\prime}\right)\left|\vec{v}-\vec{v}_{f}^{\prime}\right|
\end{gathered}
$$


After calculations involving Christoffel symbols one arrives at the following expressions for the tensors given above (symmetry is assumed about $\psi$ )

$$
\begin{gathered}
T_{a}^{1}=\frac{\partial h}{\partial v}+\frac{v_{\varphi}}{v} \frac{\partial h}{\partial v_{\varphi}} \\
T_{a}^{2}=0 \\
T_{a}^{3}=\frac{v_{\varphi}}{v} \frac{\partial h}{\partial v}+\frac{\partial h}{\partial v_{\varphi}} \\
S^{11}=\frac{\partial^{2} g}{\partial v^{2}}+2 \frac{v_{\varphi}}{v} \frac{\partial^{2} g}{\partial v \partial v_{\varphi}}+\frac{v_{\varphi}^{2}}{v^{2}} \frac{\partial^{2} g}{\partial v_{\varphi}^{2}} \\
S^{13}=S^{31}=\frac{v_{\varphi}}{v} \frac{\partial^{2} g}{\partial v^{2}}+\left(1+\frac{v_{\varphi}^{2}}{v^{2}}\right) \frac{\partial^{2} g}{\partial v \partial v_{\varphi}}+\frac{\partial_{\varphi}^{2} g}{\partial v^{2}}+\frac{\partial^{2} g}{\partial v_{\varphi}^{2}}+\frac{\left(v^{2}-v_{\varphi}^{2}\right)}{v^{3}} \frac{\partial g}{\partial v}+2 \frac{v_{\varphi}}{v} \frac{\partial^{2} g}{\partial v_{\varphi}^{2}} \\
S^{23}=S^{32}=0 \\
\frac{1}{v\left(v^{2}-v_{\varphi}^{2}\right)} \frac{\partial g}{\partial v}
\end{gathered}
$$

Thus, employing the relations

$$
\begin{gathered}
\left(f T_{a}^{\mu}\right)_{{ }^{\prime} \mu}=\sqrt{a}^{-1} \frac{\partial}{\partial q^{\mu}}\left(\sqrt{a} f T_{a}^{\mu}\right) \\
\left(f S_{a}^{\mu \nu}\right)_{{ }^{\prime} \mu \nu}=\sqrt{a}^{-1} \frac{\partial^{2}}{\partial q^{\mu} q^{\nu}}\left(\sqrt{a} f S^{\mu \nu}\right)+\sqrt{a}^{-1} \frac{\partial}{\partial q^{\mu}}\left(\sqrt{a} \Gamma_{\omega \mu}^{\nu} f S^{\mu \omega}\right)
\end{gathered}
$$

with the $\Gamma$ symbol denoting a Christoffel symbol of the second kind, the Fokker-Planck equation consists of terms 


$$
\left(f T_{a}^{\mu}\right)^{\prime} \mu=\frac{1}{v}\left(\frac{\partial}{\partial v}\left(v f \frac{\partial h}{\partial v}+v_{\varphi} f \frac{\partial h}{\partial v_{\varphi}}\right)+\frac{\partial}{\partial v_{\varphi}}\left(v_{\varphi} f \frac{\partial h}{\partial v}+v f \frac{\partial h}{\partial v_{\varphi}}\right)\right)
$$

and

$$
\begin{aligned}
\left(f S_{a}^{\mu \nu}\right)_{\prime \mu \nu}= & \frac{1}{v} \frac{\partial^{2} g}{\partial v^{2}}\left(v f S_{a}^{11}\right)+2 \frac{\partial^{2} g}{\partial v \partial v_{\varphi}}\left(v f S_{a}^{13}\right)+\frac{\partial^{2} g}{\partial v_{\varphi}^{2}}\left(v f S_{a}^{33}\right) \\
& +\frac{1}{v} \frac{\partial}{\partial v}\left(v f\left[\frac{-\left(v^{2}-v_{\varphi}^{2}\right)}{v^{3}} \frac{\partial^{2} g}{\partial v_{\varphi}^{2}}-\frac{2}{v^{2}} \frac{\partial g}{\partial v}\right]\right)
\end{aligned}
$$

Most terms involving $S^{22}$ vanish due to the further derivations with respect to the coordinate of symmetry, $\psi$, but one of them is retained in the last term in Eq.(19), therein written explicitly. The diffusion coefficients in transformations between curvilinear coordinate systems may now be identified using

$$
\begin{gathered}
\frac{1}{\Gamma_{a}}<\Delta v^{\mu}>_{a}=T_{a}^{\mu}-\frac{1}{2} \Gamma_{\omega \tau}^{\mu} S^{\omega \tau} \\
\frac{1}{\Gamma_{a}}<\Delta v^{\mu} \Delta v^{\nu}>=S_{a}^{\mu \nu}
\end{gathered}
$$

It is convenient to treat the problem in energy-angular momentum - space such that the diffusion coefficients just derived have to be transformed to the new velocity variables $E=\frac{1}{2} v^{2}+\phi(\rho, z)$ and $J_{\mathrm{z}}=\rho v_{\varphi}$. This can be accomplished by using the following simple formula:

$$
\begin{gathered}
<\Delta E>=E_{{ }^{\prime} \mu}<\Delta v^{\mu}>+\frac{1}{2} E_{{ }^{\prime} \mu \nu}<\Delta v^{\mu} \Delta v^{\nu}> \\
<\Delta J_{z}>=J_{{ }^{\prime} \mu}<\Delta v^{\mu}>+\frac{1}{2} J_{{ }^{\prime} \mu \nu}<\Delta v^{\mu} \Delta v^{\nu}> \\
<(\Delta E)^{2}>=E_{{ }^{\prime}} E_{{ }^{\prime}}<\Delta v^{\mu} \Delta v^{\nu}> \\
<\left(\Delta J_{z}\right)^{2}>=J_{{ }^{\prime}} J_{{ }^{\prime} \nu}<\Delta v^{\mu} \Delta v^{\nu}> \\
<\Delta E \Delta J_{z}>=E_{{ }^{\prime} \mu} J_{{ }^{\prime}}<\Delta v^{\mu} \Delta v^{\nu}>
\end{gathered}
$$

If an isotropic background distribution $f\left(v_{f}\right)$ is assumed, integrals $h$ and $g$ are easily simplified. For this purpose, the velocity $\vec{v}$ is expressed in terms of velocities $\left(\vec{u}+\rho \Omega \vec{e}_{\varphi}\right)$ in the corotating frame. $\Omega$ is the angular velocity of the corotating frame. Thus, $f(u)$ is 
isotropic and the derivatives of $h$ and $g$ with respect to $v$ and $v_{\varphi}$ must be transformed to those with respect to $u$ only. It leads

$$
\begin{gathered}
<\Delta E>=\left(u+\frac{J_{z} \Omega}{u}-\frac{\rho^{2} \Omega^{2}}{u}\right) \frac{\partial h}{\partial u}+\frac{1}{2} \frac{\partial^{2} g}{\partial u^{2}}+\frac{1}{u} \frac{\partial g}{\partial u} \\
<\Delta J_{z}>=\left(\frac{J_{z}}{u}-\frac{\rho^{2} \Omega}{u}\right) \frac{\partial h}{\partial u} \\
<(\Delta E)^{2}>=\left(u^{2}+2 J_{z} \Omega-2 \rho^{2} \Omega^{2}+\frac{J_{z}^{2} \Omega^{2}}{u^{2}}-\frac{2 J_{z} \rho^{2} \Omega^{3}}{u^{2}}+\frac{\rho^{4} \Omega^{4}}{u^{2}}\right) \frac{\partial^{2} g}{\partial u^{2}} \\
+\left(\frac{\rho^{2} \Omega^{2}}{u}-\frac{J_{z}^{2} \Omega^{2}}{u^{3}}+\frac{2 J_{z} \rho^{2} \Omega^{3}}{u^{3}}-\frac{\rho^{4} \Omega^{4}}{u^{3}}\right) \frac{\partial g}{\partial u} \\
<\left(\Delta J_{z}\right)^{2}>=\left(\frac{J_{z}^{2}}{u^{2}}+\frac{\rho^{4} \Omega^{2}}{u^{2}}-\frac{2 J_{z} \rho^{2} \Omega}{u^{2}}\right) \frac{\partial^{2} g}{\partial u^{2}} \\
+\left(\frac{-J_{z}^{2}}{u^{3}}-\frac{\rho^{4} \Omega^{2}}{u^{3}}+\frac{\rho^{2}}{u}+\frac{2 J_{z} \rho^{2} \Omega}{u^{3}}\right) \frac{\partial g}{\partial u} \\
+\left(\frac{\rho^{2} \Omega}{u}+\frac{2 J_{Z} \rho^{2} \Omega^{2}}{u^{3}}-\frac{J_{z} \Omega}{u^{3}}-\frac{\rho^{4} \Omega^{3}}{u^{3}}\right) \frac{\partial g}{\partial u} \\
<\Delta \Delta J_{z}>=\left(J_{z}-\rho^{2} \Omega+\frac{J_{z}^{2} \Omega}{u^{2}}-\frac{2 J_{z} \rho^{2} \Omega^{2}}{u^{2}}+\frac{\rho^{4} \Omega^{3}}{u^{2}}\right) \frac{\partial^{2} g}{\partial u^{2}}
\end{gathered}
$$

The Fokker-Planck equation is usually recast in flux conservation form:

$$
\frac{D f}{D t}=-\frac{\partial F_{E}}{\partial E}-\frac{\partial F}{\partial J_{z}}
$$

where the orbit average is still not applied. The term on the left hand side represents the Vlasov-term of the full collisional Boltzmann equation. The fluxes $F$ are given by

$$
\begin{gathered}
F_{E}=-D_{E E} \frac{\partial f}{\partial E}-D_{E J_{z}} \frac{\partial f}{\partial J_{z}}-D_{E} f \\
F_{J_{z}}=-D_{J_{z} J_{z}} \frac{\partial f}{\partial J_{z}}-D_{J_{z} E} \frac{\partial f}{\partial E}-D_{J_{z}} f
\end{gathered}
$$

Comparing these equations with the original form (e.g. Spitzer 1987) the flux coefficients $D$ may be identified to 


$$
\begin{aligned}
& D_{E E}=\frac{1}{2}<(\Delta E)^{2}>; \\
& D_{E J_{z}}=\frac{1}{2}<\Delta E \Delta J_{z}>; \\
& D_{J_{z} J_{z}}=\frac{1}{2}<\left(\Delta J_{z}\right)^{2}>; \\
& D_{J_{z} E}=\frac{1}{2}<\Delta E \Delta J_{z}>; \\
& D_{E}=-<\Delta E>+\frac{1}{2} \frac{\partial}{\partial E}<(\Delta E)^{2}>+\frac{1}{2} \frac{\partial}{\partial J_{z}}<\Delta E \Delta J_{z}>; \\
& D_{J_{z}}=-<\Delta J_{z}>+\frac{1}{2} \frac{\partial}{\partial J_{z}}<\left(\Delta J_{z}\right)^{2}>+\frac{1}{2} \frac{\partial}{\partial E}<\Delta E \Delta J_{z}>
\end{aligned}
$$

Inserting the expressions for the diffusion coefficients given above, one obtains finally the desired flux coefficients for the axially symmetric application with rotation.

$$
\begin{gathered}
D_{E}=4 \pi\left(\frac{J_{z} \Omega}{u}-\frac{\rho^{2} \Omega^{2}}{u}+u\right) F_{2}(u) \\
D_{J_{z}}=4 \pi\left(\frac{J_{z}}{u}-\frac{\rho^{2} \Omega}{u}\right) F_{2}(u) \\
D_{E E}=\frac{4 \pi}{3}\left(2 u J_{z} \Omega-u \rho^{2} \Omega^{2}+u^{3}\right) E_{1}(u)+2 \pi\left(u \rho^{2} \Omega^{2}-\frac{J_{z}^{2} \Omega^{2}}{u}+\frac{2 J_{z} \rho^{2} \Omega^{3}}{u}-\frac{\rho^{4} \Omega^{4}}{u}\right) F_{2}(u) \\
+2 \pi\left(\frac{2 u^{3}}{3}+\frac{4}{3} u J_{z} \rho-\frac{5}{3} u \rho^{2} \Omega^{2}+\frac{J_{z}^{2} \Omega^{2}}{u}+\frac{\rho^{4} \Omega^{4}}{u}\right) F_{2}(u) \\
D_{J_{z} J_{z}}=\frac{4 \pi}{3} E_{1}(u)+2 \pi\left(u \rho^{2} \frac{J_{z}^{2}}{u}-\frac{\rho^{4} \Omega^{2}}{u}+\frac{2 J_{z} \rho^{2} \Omega}{u}\right) F_{2}(u) \\
\left.+2 \pi\left(\frac{J_{z}^{2}}{u}+\frac{\rho^{4} \Omega^{2}}{u}-\frac{2 J_{z} \rho^{2} \Omega}{u}\right)-\frac{1}{3} u \rho^{2}\right) F_{4}(u) \\
+2 \pi\left(\frac{2}{3} u J_{z}-u \rho^{2} \Omega-\frac{2 J_{z} \rho^{2} \Omega^{2}}{u}+\frac{\rho^{4} \Omega^{3}}{u}+\frac{J_{z}^{2} \Omega}{u}\right) F_{4}(u) \\
D_{E J_{z}}=\frac{4 \pi}{3} u J_{z} E_{1}(u)+2 \pi\left(u \rho^{2} \Omega+\frac{2 J_{z} \rho^{2} \Omega^{2}}{u}-\frac{J_{z}^{2} \Omega}{u}-\frac{\rho^{4} \Omega^{3}}{u}\right) F_{2}(u) \\
{ }^{2}(3)
\end{gathered}
$$

The functions $E_{i}$ and $F_{i}$ are constituent parts of the Rosenbluth potentials and their derivatives

$$
F_{i}=\frac{1}{u^{i}} \int_{0}^{u} u^{\prime i} f\left(u^{\prime}\right) d u^{\prime}
$$




$$
E_{i}=\frac{1}{u^{i}} \int_{u}^{\infty} u^{\prime i} f\left(u^{\prime}\right) d u^{\prime}
$$

Using the background distribution function given in (Eq. 3.37), these functions are evaluated for each position in the meridional plane, when the local density, mean particle velocity and mean particle angular velocity are given. Following, the orbit-average needs to be applied to the flux coefficients and transformed to $(X, Y)$-coordinates, as explained in Sect. (3.2.1), in order to calculate Equations (3.19) to (3.23). 


\section{Acknowledgments}

I would like to thank my advisor, Prof. Dr. Rainer Spurzem (ARI/ZAH). His willingness to support my work and his guidance throughout my studies allowed me to develop my skills as a researcher within a supportive team environment. I thank him for that opportunity. I would like to thank Prof. H.M.Lee (Seoul National University) and his group of work, specially Dr. E. Kim, for his support during my stay in Seoul. At ARI-Heidelberg, I would like to thank Dr. Miguel Preto, for interesting technical, and sometimes not so technical discussions with regard to this research, and general science. To Dr. Gabor Kupi, for his support and disposition. Further I thank Patrick Glaschke, Andreas Ernst, Pau Amaro, Christoph Eichhorn and all the members of our 'Stellar Dymamics' group at the ARI/ZAH, who made possible a nice atmosphere of work during the last years. I greatly appreciate their time and input to this dissertation. Finally, I must express my appreciation to the many friends outside of my studies who have helped to relieve the sometimes stressful solitude of graduate school. 



\section{Vita}

José Fiestas was born in Lima, Perú, on November 5, 1969, the son of J. Reynaldo Fiestas and Sila Iquira de Fiestas. After graduating in 1987 from 'San Andres' High School, Lima, he attended the University of Lima, where he received a Bachelor of Engineering degree, in 1992. After 3 years of work experience in a textile company in Lima, he decided to start, in 1995, studies of Astronomy at the University of Heidelberg, in Germany. His undergraduate studies, were self-financed through different jobs. In 2002, he started as a doctoral student at the Astronomisches Rechen-Institut in Heidelberg, where he completed his Doctor of Philosophy degree in 2005. In the future, José hopes to pursue a professional career in science. 